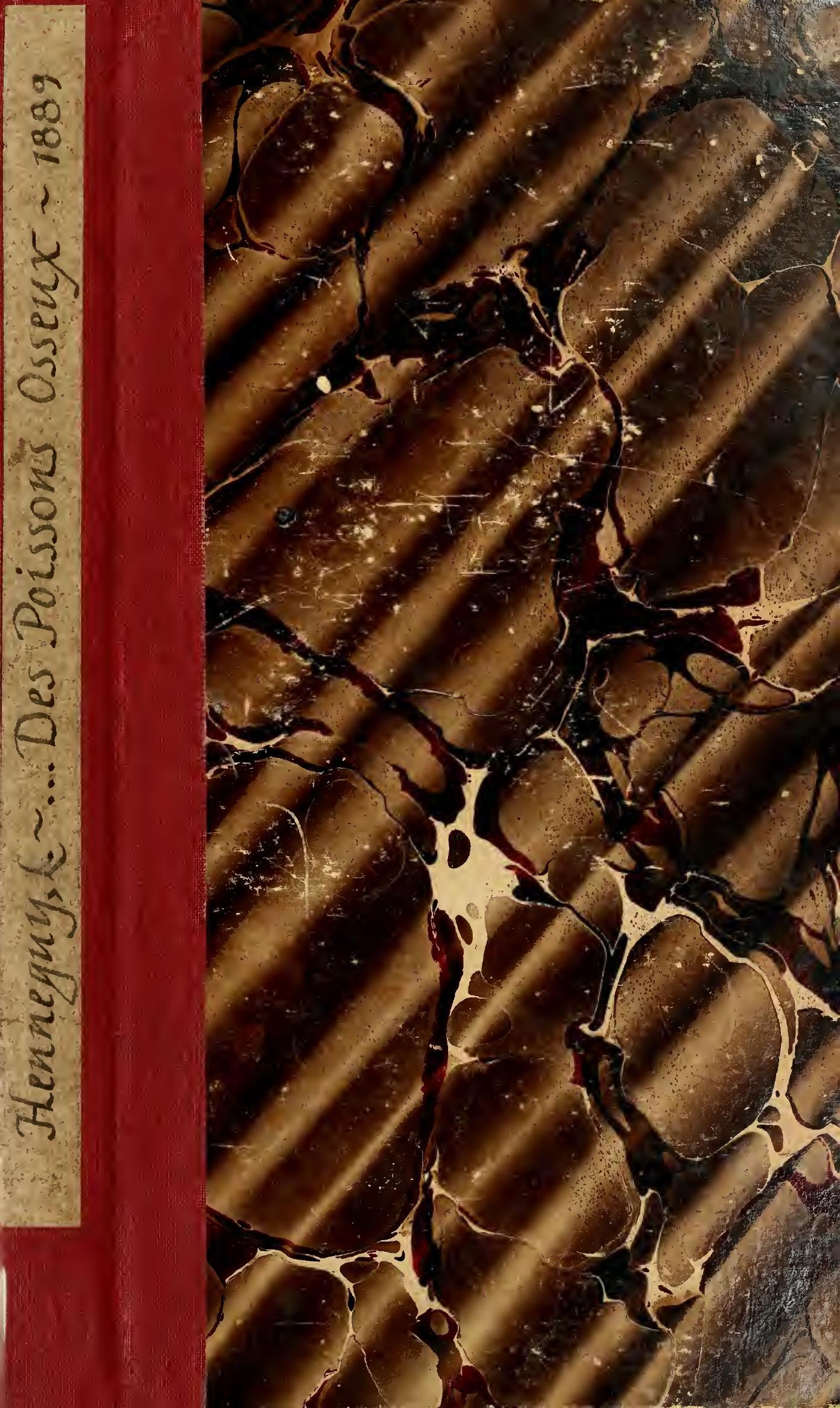


Alex. Agassiz.

afibrarn of the eftluscum

$\mathrm{OF}$

\section{COMPARATIVE ZOÖLOGY,}

AT HARVIRD COLLEGE, CAJIBRIDGE, ILASS.

fromined bo pribate substription, in 1 s61.

Deposited by ALEX. AGASSIZ.

No.12,359
opr. 22. 1890 


Série $A, n^{0} 116$

No IDORLRE

T Fì̀ S E S

651.

\section{A LA FAGULTÉ DES SGIENGES DE PARIS}

POUR OBTEXIR

LE GRADE DE DOCTEUR ES SCIENCES NATURELLES

PAR

\section{FÉLIX HENNEGUY}

Docteur en médecine,

Préparateur an College de France, Membre de la Société de Biologie.

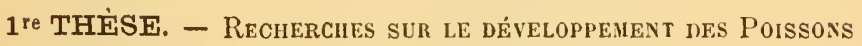
osseux. Eubryogénie de la Truite.

2e THĖSE. - Propositions donNÉEs par la Faculté.

Soutenues le 12 Anuil 1889, devant la Commission d'examen

MM. HÉBERT. . . . . . . Présıdent.

DE LACAZE-DUTHIERS.
BONNIER. . . . Examinateurs.

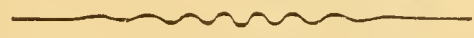

PARIS

ANCIENNE LIBRAIRIE GERMER BAILLIERE ET C C $^{\text {i }}$ FÉLIX ALCAN, ÉDITEUR

108, BOULEVARD SAINT-GERMAIN, 108

1889 


\section{ACADÉNIE DE PARIS}

\section{FACULTÉ DES SCIENCES DE PARIS}

Doyen. . . . . . HÉBERT, Professcur. . . . Géologie.

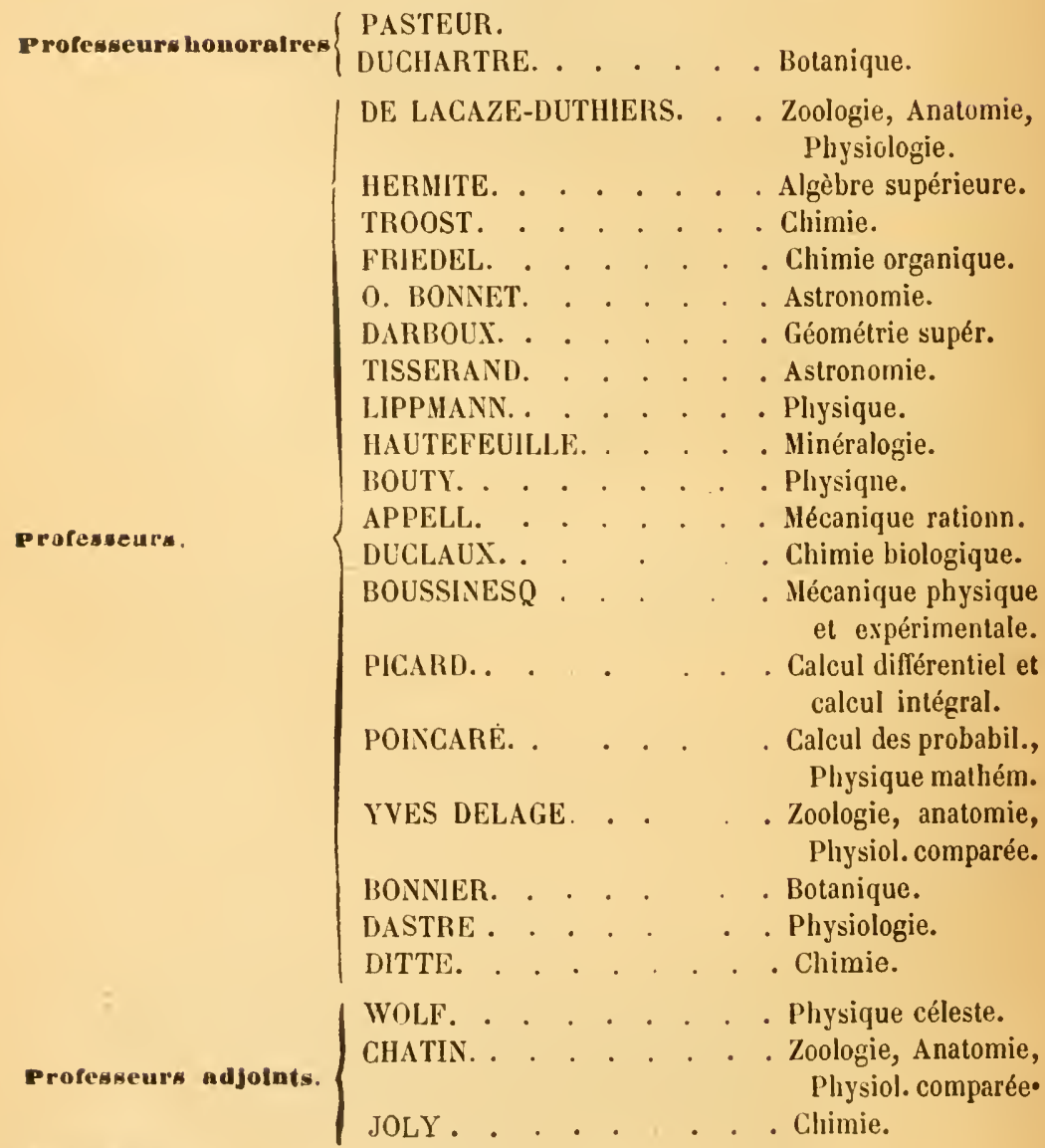

secrétalre. .... PHILIPPOX. 


\title{
A MON MAîTRE
}

\author{
M. E. G. BALBIANI \\ Professour d'Embryogénie comparée au Collège de France.
}

TÉMOIGNAGE DE VIVE RECONNAISSANCE ET DE RESPECTUEUSE AFFECTION. 



\title{
REGHERGHES
}

SUR LE

\section{DÉLELOPPENENT IDES POISSOLS OSSELY}

\author{
EMBRYOGENIE DE LA TRUITE
}

INTroductox.

Les travaux auxquels a donné lieu l'embryogénie des Poissons osseux sont déjà tellement nombreux, qu'il paraitra peutêtre superflu de faire de nouvelles recherches sur ce sujet, et surtout de prendre comme objet d'étude, la Truite, l'un des Poissons dont le dévelopyement a été le plus souvent suivi. Si l'on examine cependant arec soin les mémoires publiés jusqu’à ce jour sur la question, on est frappé du désaccord qui existe entre les auteurs sur des points qui ont une très grand. importance, non seulement en ce qui concerne le développement particulier des l'oissons, mais encore au point de vue de l'umbryogénie générale; telles sont, par exemple, l'origine des fenillets blastodermiques, la lormation dı système nerveux, dn cour, dı sang, etc.

Depuis lestravaux de Vogt (191) et de Lereboullet (112), qui, hien que déjà anciens, peuvent ètre encore consultés avec fruit pour les stades avancés, l'importint mémoire d'OEllacher (123) était à peu près le seul, en 1880, lorsque je commençai mes recherches, que nous avions sur le développement des Salmonides. Jai done cru, à cette époque, quil serait utile de reprendre à nouvean l'étude de l'embryogénie de la Truite, à laide des procédés que la technique moderne mettait à ma disposition. Depuis lors, il est vrai, ont parn les observations de C. K. Hoffmann (89), Ziegler (200) et Goronowitsch (57), mais Jes résultats obtenus par ess auteurs concordant peu entre eux ot différant sur plusieur's joints de ceux auxquels j’étilis déjà 
arrivé, loin de me décourager, m'engagèrent à poursuivre mon étude.

La fucilité que javais de me procurer des œufs de Truite à tous les états de développement dans les aquariums établis par Coste, au Collège de France, m’a déterminé à choisir cette espèce de Poisson, comme objet de mes recherches. J'ai fait également quelques obserrations sur l'Epinoche et la Perche.

J'arais primitirement l'intention de suivre d'une manière complète le développement de la Truite, depuis la fécondation de l'cuf jusqu'à l'éclosion; mais, outre que les stades avancés, déjà bien décrits par Vogt et Lereboullet, ne présentent pas un très grand intérêt au point de vue embryogénique, je n’ai pas tardé à reconnaitre que leur étude, faite avec autant de détails que le comporte celle des premiers stades, exigeait un travail de longue halcine, et pouvait donner lieu à plusieurs mémoires distincts. Bien que possédant des préparations nombreuses relatives à tous les stades du développement de la Truite, jai donc dû limiter mes recherches aux premières phases de ce développement. Je me suis arrêté dans ce travail au moment où le blastoderme a recouvert la totalité du vitellus. Ce stade caractéristique est très important pour la Truite, car il correspond à la formation des organes les plus essentiels du système nerveux central, des yeux, des résicules auditives, des premières fentes branchiales, de l'intestin, du canal de Wolff et du coeur.

Ce travail, commencé en 1880, n’a pu, par suite de circonstances indépendantes de ma volonté, être publié plus tòt, mais j’en ai déjà indiqué les principaux résultats dans des communications préalables faites à l'Académie des sciences, à la Société de Biologie, et à la Société philomathique (67 à 76).

L'époque de la reproduction pour les Truites du Collège de France commence dans les premiers jours de novembre : ces animaux, élevés en captivité depuis plusieurs générations, semblent avoir perdu l'instinct de frayer; abandonnées à cllesmêmes, les femelles ne se débarrassent pas de leurs œufs, qui restent dans la cavité abdominale, sont en partie résorbés ou finissent pour amener la mort de l'animal. Aussi chaque année, du mois de novembre ì la fin de janvier, on pratique la fécondation artificielle des ceufs, en suirant les règles établies 
par Coste dans ses Instructions pratiques sur la pisciculture. Depuis plusieurs années on emploie cependant de préférence la méthode russe qui donne un peu plus grand nombre d'œufs fécondés que la méthode indiquée par Coste. La méthode russe consiste, comme on sait, à recevoir les œufs à sec dans un récipient, à faire tomber dessus une certaine quantité de laitance et à ajouter ensuite de l'eau pour opérer la fécondation.

La durée de l'incubation est très variable et dépend de la température; elle peut varier de 33 à 100 jours. Cette différence considérable dans la durée de l'incubation ne permet pas de désigner l'àge d'un œuf ou d'un embryon, comme on le fait par exemple pour le P'oulet, par le nombre d'heures ou de jours qui le sépare de la ponte. Un œuf pondu depuis vingt jours peut renfermer un embryon dont le développement ne sera pas plus avancé que celui d'un embryon contenu dans un autre œuf ayant seulement huit jours d'incubation. C'est pour eette raison que, dans mon travail, je n'indiquerai pas l'âge des embryons; à l'exemple de Balfour, j'ai établi un certain nombre de stades artificiels, correspondant à des périodes déterminées du développement et caractérisées, soit par une modification dans la forme extérieure, soit par l'apparition d'un nouvel organe. Cette méthode est la seule qui permette de rendre comparables les travaux faits, par des auteurs différents, sur le développement d'un animal dont la durée de l'évolution embryonnairc varie avec les circonstances extérieures.

Ce n’cst qu'après de longs tâtonnements et après avoir sacrifié un assez grand nombre d'œufs que je suis parvenu à trouver des modes de préparation de l'embryon permettant d'obtenir de bomnes coupes, et n'altérant pas les éléments histologiques. J'insigterai un peu longuement sur les métlıodes que j’ai employées, parce qu'en embryologie, plus peut-être qu'en toute autre science biologique, la technique joue un ròle des plus importants. Je montrerai par la suite que beaucoup des divergences äopinions qui existent entre les auteurs peuvent s'expliquer lacilement en tenant compte des procédés qu'ils ont employés pour fixer et durcir l'embryon.

Au début de mes recherches, je plaçais les œufs dans une solution d'acide chromique à $\frac{1}{100}$ et je les y laissais pendant quelques jours. La forme extérieure de l'embryon était assez bien 
conservée, du moins pour les premiers stades du développement, mais les cellules étaient déformées et j'ai dû rejeter la plupart des préparations obtenues par ce mode de durcissement. Pour les stades plus avancés, lorsque l'embryon est dejà formé, l'acide chromique amène des déformations considérables de l'embryon qui se trouve comprimé entre la coque inextensible de l'cuf et la masse vitelline solidifiée.

Le procédé suivant m'a donné de meilleurs résultats. Les œufs étaient mis pendant quelques minutes dans de l'eau fortement acidulée par de l'acide acétique, jusqu'à ce que l'embryon devint bien visible, puis dans une solution d’acide chromique à $\frac{1}{100}$. Au bout de trois jours, les œufs étaient retirés de l'acide clıromique et jenlevais leur chorion au moyen de petites pinces fines, en aỵant soin d’attaquer le chorion du côté opposé à l'embryon. Après un séjour de vingt-quatre heures dans l'eau, les œufs étaient mis dans laleool à $90^{\circ}$, puis dans l'alcool absolu; les embryons ainsi préparés ne sont pas déformés et leurs éléments histologiques sont beaucoup plus nets qu'avec l'acide chromique seul.

Le durcissement de l'embryon in situ, reposant sur le vitellus et recouvert par la capsule de l’œuf, présente des inconvénients. La masse vifclline dans l'acide chromique acquiert une dureté beaucoup plus grande que celles des tissus embryonnaires, et devient très cassante; il est alurs très difficile de pratiquer des coupes à travers la masse totale de l'œuf.

J'ai tronvé deux procédés qui permettent d'extraire des ceufs les germes et les embryons avee la plus grande facilité et sans leur faire subir la moindre altépation. Le premier consiste à placer l'œuf, pendant quelques minutes, dans une solution d'acide osmique à $\frac{1}{100}$, jusqu'à ce qu'il ait acquis une couleur brune claire, puis dans un petit vase renfermant de la liqueur de Yüller. On ouvre l'œuf dans ce liquide arec une paire de ciseaus fins. Le vitellus, qui se coagule immédiatement au contact de l'eau, se dissout au contraire dans le liquide de Hüller, tandis que la couche corticale et le germe fixés par l'acide osmique peuvent ître extraits de la capsule de l'œuf. On laisse le germe ou l'embryon pendant quelques jours dans la liqueur de Müller, puis. apros larrile lave soigneusement pour enlever le bichromate de potasse. on le passe par des alcools de plus en plus forts. Ce 
procédí, qui réussit très hien pour les premiers stades du déreloppenent, nest pas aussi bon lorsque l'embryon est issez avancé : lans ce cas, en effet, l'acide osmique ne le pénètre pas suffisamment. Sa surface seule est bien fixéc. L'acide osmique est excellent pour bien mettre en évidence la réflexion des bords du blastoderme et les limites de cliaque feuillet.

Le sccond procédé est cehi qui donne les meilleurs résultats et que jai presque constamment employé. L’onf est plongé pendant une dizaine de minutes dans le liquide de Kleinenberg additionné de 10 pour 100 d'acide acétique eristallisable. Puis jl est ouvert dans de l'eau renfermant 10 pour 100 d'acide acétique, liquide dans lequel le vitellus est soluble, et on extrait l'embryon. Celui-ci est mis ensuite pendant quelques heures lans le liquide de kleinenberg pur, puis dans l'aleool ì $60^{\circ}$, $7 \dddot{3}^{\circ}, 90^{\circ}$ et finalement dans l'alcool absolu. Cette méthode conserve aux éléments toute leur intégrité, elle n'amène aucune altération dans la forme de l'umbryon. Dans toutes mes préparations jai trouvé toutes les fignres eytodiérétiques intactés, tandis qu'arec les antres procédés elles sont la plupart du temps altérées.

L'embryon durci et déshydraté par l'alcool absolu est inclus dans le collodion ou dans la paraffine, l'après les méthodes ordinaires, et coupé soit avec le mierotome de Jung, soit avec le mierotome à bascule de Cambridge. L'embryon peut être coloré en masse, avant l'inclusion, soit par le carmin boraté alcoolique, soit par le carmin aluné acide, soit frar l'hématoxyline. On obtient ainsi de très belles préparations en colorant les coupes, préalablement fixées au porte-objet, par les couleurs d'aniline, la safranine, ou le violet de gentiane jar la méthode de Bizzozzero.

Pour l'étude des formes extérieures, les œufs dureis par l'acide chromique et dépouillés de leur capsule, sont ceux qui conviennent le mieux. J'ai fait également pour les différents stades des préparations de germes et d'embryons entiers, détachés avec soin du vitellus el montés dans le baume, après coloration au carmin. Ces préparations sont indispensables paree qu'elles montrent des détails qui échappent sur les coupes, et qui sont absolument invisibles lorsqu'on se contente d'exaniner à la lumière directe les embryons dureis. J'ajouterai que 
j'ai eu soin, pour chaque stade, de varier les modes de préparation de l'embryon, afin de contrôler les résultats obtenus par la méthode qui m'a paru la meilleure.

\section{PREMIERE PARTIE}

\section{I. - Constitution de l'oeuf xon fécondé}

La constitution de l'œuf des Salmonides est aujourd'hui bien connue, grâce aux recherches de Vogt (191), Lereboullet (112), OEllacher (123) et His (82); mes propres observations chez la Truite confirment en grande partie celles de His chez le Saumon.

L'œuf de la Truite, pris dans la cavité abdominale lorsqu'il vient de se détacher de l'ovaire, est assez volumineux, il mesure de 4 à 6 millimètres de diamètre. Sa couleur est jaune citron clair, et il est alors à peu près transparent; sa consistance est assez molle, mais il devient rapidement dur et très élastique lorsquill a séjourné quelque temps dans l'eau. Ce changement de consistance est dû, ainsi que Miescher (118) l'a démontré, à une absorption d'eau qui rend l'œuf turgescent et tend fortement la capsule. Hiescher a trouvé, en effet, qu'un couf de Saumon, du poids de 127 milligrammes au sortir de la cavité abdominale, pesait 133 milligr. après quelque temps d'immersion dans l'eau; mais nous verrons plus loin que l'eau absorbée ne pénètre pas dans l'intérieur de l'œuf.

Si l'on fait une coupe d'un ouf de Truite, pris dans la cavité abdominale et durci, on trouve en allant de la périphérie vers le centre: $1^{\circ}$ une membrane épaisse (capsule de l'œuf); $2^{\circ}$ une zone périphérique protoplasmique $; 3^{\circ}$ une masse centrale (vitellus nutritif).

La membrane externe est assez épaisse et mesure de 30 à $3 \check{3}$; elle est résistante et élastique. Examinée au microscope, elle se montre traversée par des canalicules très fins, qui ont été découverts par J. Müller (120).

Suirant Stochmann (180), les canalicules de cette membrane 
n'auraient pas des limites bien nettes et présenteraient une série de dentelures inégales; entre les canalicules se trouve une substance homogène, Owsjannikow (125) admet dans l'en veloppe de l'œuf trois couches : une mince membrane externe finement ponctuée, une couche moyenne striée et une membrane mince et transparente. La surface de l'œuf serait recouverte de très petites proéminences. Malgré l'emploi de forts grossissements, je n'ai pu voir les trois couches déerites par Owsjannikow, ni les dentelures des canalieules indiquées par Stochmann.

Lienveloppe de l'œuf a reçu des noms différents suivant les auteurs. Vogt (191) l'a appeléc membrane coquillière, nom évidemment impropre, car cette enveloppe existe déjà dans le follicule ovarien, tandis que la membrane coquillère des œufs des Sauropsides ne se forme que dans l'oviducte. Lenckart(114) et Lereboullet (112) lui donnent le nom de chorion et admettent qu'clle est une production vitelline. J. Müller (120) la désigna sous le nom de membrane vitelline. Allen Thomson (188) l'assimila avec raison à la zone pellucide de l'œuf des Mammifères. C'est aussi sous le nom de membrane vitelline que la désignent Kœlliker (97), Gegenbaur (53), Waldeyer (192), Ed. Van Beneden (19) et Kolessnikow (95). OEllacher (123) admet que c'est un chorion et décrit une membrane vitelline au-dessous de cette première enveloppe. His (82) appelle la membrane striée, capsule de l'cuf, et C. K. Hoffmann (89) lui donne le nom de zone radiée. Nous préférons, ainsi que le fail M. le professeur Balbiani (7), conserver la dénomination 'de His et appeler capsule l'enveloppe externe de l'cuf; ce nom a l'avantage de ne rien préjuger de la nature et de l'origine de cette membrane, qui a été considérée par presque tous les observateurs comme un produit de différenciation du vitellus, mais que Waldeyer regarde comme un produit de sécrétion du follicule ovarique. C. K. Hoffmann, qui a étudié récemment l'ovogínèse chez plusicurs Poissons, se range à l'opinion la plus généralement adoptée et admet que la capsule de l'œuf est un produit du vitellus et est par consérquent une véritable membrane vitelline. N'ayant pas fait de recherehes personnelles sur ce sujet, je ne saurais me prononcer en faveur de l'une ou de l'autre opinion. 
La capsule de l'œuf présente toujours une petile ouverture, micropyle, qui a été découverte par Doyère (38), en 1850, sur l'œuf du Syngnathus ophidium et qui a été vue ensuite par Carl Bruch (28), en 1855, chez le Sammon, la Truite, la Carpe et le Brochet. His (82) a bien étudié la forme du imicropyle chez les Salmonides; celui du Saumon diffère un peu de celui de la Truite. Cette ouverture est située au fond d'une petite dépression en forme de cuvette, qu'on aperçoit facilement à la surface externe de la capsule surtout sur des œufs qui n’ont pas encore été immergés dans l'eau. A un faible grossissement, le micropyle apparaît au fond de la euvette comme un petit point noir. (Planches, fig. 1.) Si l'on pratique une coupe à travers la capsule au niveau du mieropyle, on constate que la paroi interne de la capsule fait saillic à ce niveau dans l'intérieur de l'œuf. Chez le Sammon, au fond de la cuvette, il existe une sorte de petit cratère assez érasé, qui se termine par un canal très étroil; chez la Truite, ce cratère est beaucoup moins large; ses bords sont taillés à pic, et le canal qui le termine est plus large que dans le micropyle du Saumon. Le micropyle de l'Epinoche se rapproche de celui du Siumon, mais il en diffère en ce que le bord externe de la cuvette présente plusieurs plis saillants.

C. K. Hotfmanu (89) a mesuré avec soin le diamètre du micropyle l'œuf de quelques Poissons : il a trouvé pour le Hareng $0,2^{00} \mu$; chez le Crenilabrus griseus, l'orifiee interne mesure $0,2 \mu$; la tête du spermatozoïde du Hareng n'a que $2 \mu$, d'après liupfer ; celle du Crenilabrus mesure $0,17 \mu$ d'après Iloffmann. Il ne peut done passer qu'un seul spermatozoïde à la fois par le canal micropylaire, comme His l'avait déjà établi pour le Sammon.

La position du mieropyle est fixe. Cet orifice est toujours situé, sur l'œuf pris au moment de la ponte, au-dessus du germe. Après la ponte, le vitellus se rétracte et peut exécuter un mouvement de rotation dans l'intérieur de la capsule, de sorte que les rapports entre le micropyle et le germe sont changés. Il en est de mème dans les oufs non fécondés, qui ont séjourné pendant quelque temps dans l'eau.

Au-dessous de la capsule de l'œuf, se trouve une couche déerite par Lereboullet (112) ehez la Truite. "Des œufs coagulés immédiatement après la fécondation offrent, dit cet auteur, la 
composition suivante: il eviste ì l'un des piles de l'uruĺ, une prillicule nembrancuse, amorphe, fenètrée, c'est-ì-ilire olliant une multitude de trous que remplissaient des vésicules graisseuses : celles-ci s’échappent pendant lia préparration de la pièce, ete. "Ilis (82) a donné à cette zone périphérique le nom de couche corticule et la décrit à peu près comme Lereboullet, mais il a constaté qu'elle entoure complètement le vitellus.

C'est dans la couche corticale, formée par une substance proloplasmique finement granuleuse, que sont contenus les gros globules, rougeitres rhez le Saumon, jaunâtres chez la T'ruite, qui donnent en partie aux œufs de ces animaux leur coloration particulière. (Planches, fig. 4, h.) Tous les auteurs, jusqu’à His, considéraient ees globules comme étant de nature graisseuse. Ilis ayant vu que les éléments augmentent de volume et pailissent quand on les met au contact de l'eau, pensait qu’ils étaient formés de lécithine; mais Miescher (118) a montré quils ne renferment pas cette substance. Les recherches que j’ai faites sur la nature des éléments d'apparence luileuse de l'œuf des Salmonides m'ont donné les résultats suivants :

Chaque globule isolé est entouré d'une mince couche de protoplasma granuleux, comme His l'avait déjà décrit et figuré; ce protoplasma est le même que celui qui constitue la couche corticale. Les éléments huileux sont beaucoup moiñs denses que l'eau et plus denses que l'alcool absolu; ils suffisent à faire surnager un germe durci à li surface de l'eau, lorsqu’ils restent attachés à la face inlérieure de ce germe. Ils sont insolubles dans l'eau; leur augmentation de volume au contact de ce liquide est due au gonflement ou à la disparition de leur enveloppe protoplasmique; ils s'étalent alors sur la lame de verre qui les supporte; ils prennent le même aspect lorsquon les comprime légèrement à l'aide d'une lamelle.

Lorsqu'on écrase des œufs de Sammon dans l'eau on voit surnager à la surface du liquide une couche huileuse, rosée, qui est composéc de globules plus ou moins gros. Les globules ne se coagulent pas sous l'influence de la chaleur dans des ceufs portés à $100^{\circ}$, ils restent liquides, mais ils renferment alors de fines granulations réfringentes dans leur intérieur.

La matière huileuse est insoluble dans la potasse; elle n'est 
pas coaguléc par les acides concentrés; clle est soluble dans l'éther et le clıloroforme lorsqu'on a déchiré mécaniquement la couche albuminoïde qui entoure les globules; elle noircit fortement sous l'influence de l'acide osmique et plus ou moins rapidement selon le volume des vésicules. Lorsqu'on traite les gouttelettes huileuses par l'alcool absolu, leur matière colorante est dissoute et elles conscrvent une teinte légèrement jaunàtre. Toutes les réactions que présentent les globules nuileux des œufs de Salmonides permettent done d'admettre que ces ẻléments sont formés d'une matière grasse spéciale. D'après Valenciennes et Frémy (190), cette matière serait une huile phosphoréc (1).

Il existe aussi dans la coucle corticale des éléments globuleux, pâles, mous, incolores et de dimension variable, que His considère comme des noyaux, en se fondant sur une analyse de Miescher qui prétend que ces corps sont constitués par de la nucléine. Ces prétendus noyaux ne se colorent pas sous l'influence des réactif́s employés d'ordinaire pour décider la présence des noyaux des cellules; ils paraissent être des vésicules formées par une substance albuminoïde moins granuleuse que celle de la couche corticale.

Au-dessous du micropyle, la couche corticale présente un épaississement assez considérable, qui constitue le germe. Celui-ci est formé par une substance très finement granuleuse, à peu près transparente, à l'état frais, et très peu consistante; aussi, lorsqu'on l'extrait de l'œuf, il s'étale en diffluant et émet dans tous les sens de nombreux prolongements, qui lui donnent l'apparence d'un Rhizopode. L'alcool et les acides coagulent le germe et la couche corticale qui deviennent beaucoup plus consistants et peuvent alors être extraits de l'œul'; mais tandis que le germe devient opaque, la couche corticale garde en partie sa transparence.

La masse centrale de l'œuf, le vitellus, est constituée par une substance hyaline, homogène et visqueuse, ne renfermant aucun élément figuré. Cette substance, au contact de l'eau fure, de l'alcool et des acides, se coagule immédiatement en devenant blanche et opaque. L'acide acétique cristallisable la

(1) D’après Miescher, l'huile rosée des œufs de Saumon ne contiendrait pas trace de phosphore (V. le Mémoire de His (8:2) p. 7). 
coagule en lui conservant sa transparence, le même acide acétique dilué dans l'eau dissout le vitellus, ainsi que je l'ai déjà indiqué; il en est de même des alcalis, de lì liqueur de Müller et de l'eau fortement salée. L'alcool, qui coagule la substance vitelline lorsqu'il est mis directement en contact avec elle, la durcit sans la rendre opaque lorsqu'il agit lentement à travers la capsule de l'œuf.

Valenciennes et Frémy (190) ont trouvé dans le vitellus des œufs de plusieurs Poissons, entre autres du Saumon, une substance particulière, à laquelle ils ont donné le nom d'ichtuline; sa composition se rapproche de celle de l'albumine, elle contient du soufre et du phosphore. Ce serait l'ichtuline qui occasionnerait en se précipitant l'opacité des œufs des Salmonides, lorsqu'on les soumet à l'action de la chaleur, de l'eau, de l'alcool, etc.

L'eau coagulant le vitellus et l'œuf, plongé dans l'eau, absorbant une certaine quantité de liquide, comme le prouvent les pesées faites par Miescher, on doit naturellement se demander pourquoi l'œuf conserve sa transparence après la ponte. Ce phénomène est dû à ce que l'eau qui pénètre dans l'œuf à travers le micropyle et les canaux poreux de la capsule, n'arrive pas jusqu'au vitellus, qui est entouré par une couche protoplasmique. L'eau s'accumule entre la capsule et la conche corticale, ainsi que le démontrent les mouvements de rotation intracapsulaires dont est susceptible la masse de l'œuf après quelque temps d'immersion dans l'eau. Les mouvements de rotation sont au contraire impossibles dans les æufs pris dans la cavité abdominale. Un autre fait qui prouve bien que e'est la couche corticale? qui protège le vitellus, c'est que, quand cette couche est rompue, ce qui arrive souvent lorsque l'œuf est soumis à une trop forte pression, le vitellus se coagule immédiatement et l'œuf devient opaque et blanc. On observe le mème phénomène quand l'œul est envahi par les moisissures; celles-ci traversent la capsule et perforent la couche corticale.

Jusquà présent nous avons considéré l'œuf pris dans la cavité abdominale ou récemment pondu mais non encore féeondé. Les anciens embryogénistes admettaient que le germe n'existe pas dans l'œuf mûr et qu'il n'apparaît qu'après la ponte. Coste (33) pensait que chez les Poissons osseux les éléments 
plastiques et les élémunts nutritifs sont mêlés dans l'œuf jusqu'au noment de la ponte et que, sous l’influence de la fécondation, il se fait un départ entre les deus sortes d'éléments, ayant pour effet de rassembler en un point de l'œuf les éléments plastiques qui constituent le germe. Telle était aussi l'opinion de Lerchoullet (112), du moins pour l'œuf des Salmonides. Car cet embryogéniste avait très bien constaté que le germe existe avant la fécondation chez la Perche et le Brochet. Yl. Gerbe (55) a également arancé récemment que ce n’est qu’à la suite de la jonte, après quelque temps de séjour dans l'eau, indépendamment de la fécondation, que le germe se manifeste dans l'œuf des Poissons osseux. His, au contraire, a observé le germe formé, peu visible il est vrai, dans l'cuf du Samon avant la ponte. Hes propres observations ont confirmé celles de His, et elles mont permis en outre de suivre le mode de formation du germe chez la Truite (70).

L'ouf ovarien mûr a une constitution tout à fait différente de celle de l'œuf pris dans la cavilé abdominale. Si l'on examine, en effet, un cuf ovarien quelque temps avant la rupture du follicule, on voit, dans la région micropylaire, la vésicule germinative située très près de la surface de l'ouf. Cette vésirule est entourée de petits globules, à contours finement granuleux, qui augmentent de volume à mesure qu'on s'éloigne de la vésicule germinative. (Planches, fig. 1 et 3.)

Les éléments granuleux sont bientòt remplacés par des vésicules plus grosses, transparentes, renfermant quelques racuoles et de petits globules réfringents se colorant en noir par l'acide osmique. Les globules granuleux sont les éléments plastiques de l'ceuf et les vésicules transparentes constituent la partie nutritive: les éléments plastiques sont done, dans l'œuf ovarien, distincts des éléments nutritifs et rassemblés autour de la vésieule germinative; on n'en trouve pas dans le reste de l'ouf.

J'ai eu l'occasion d'examiner les ovaires d'un Gymnote mort daus le laboratoire de $\mathrm{M}$. le professeur Marey; les oufs présentaient une structure des plus intéressantes et permettant de saisir la formation des éléments plastiques et vitellins. Leur contenu était formé de petites vésicules de rolume et d'aspect rariables. Les unes avaient un double contour brillant et ren- 
fermaient dans leur intérieur une vésicule plus petite, brillante, ne remplissant pas toute la eavilé. D’autres vésicules contrnaient trois ou quatre petites vésieules secondaires. Les plus grands éléments présentaient dans leur intérieur un grand nombre de petites vésieules secondaires, pressées les unes contre les autres et remplissant toutes la eavité limitée par le double contour A còté lo ces éléments il y avait des vésicules plus pàles, à simple contour, remplies de granulations très fines et présentant quelquefois un ou deux globules réfringents. (Planches, fig. :.j.) Ces derniers éléments ressemblent aux vésicules du jaune de l'œuf des Oiseaux. Enfin on voyait aussi très distinctement dans les oufs ovariens du Gymnote la vésicule germinative et la vésieule enbryogène.

Hoffmann (89) a également constaté que les coufs ovariens du Ilareng et de quelques autres Poissons de mer renferment de grains et de sphères vitellines simples ou composées, formées aux dépens du protoplasma de l'œuf.

Après la déhiscence du follicule ovarien, l’œuf tombé dans la ravité abdominale présente un tout autre aspect. La vésicule germinative a disparu; les vésicules constituant la partie nutritive se sont fusionnées et ne forment plus qu'une masse visqueuse homogène. Les globules huileux se sont rassemblés à la périphérie de l'wuf et constituent de grosses gouttelettes rétringentes de volume variable, plus nombreuses dans la région micropylaire que dans le reste. Enfin les éléments plastiques se sont aussi fusionnés en une masse finement grinuleuse, irrégulièrement étaléc au-dessous du micropyle : c'est le germe. (Plinches, fig. 2 et 4.)

En traitant l'œul par l'acile acétiqur, on voit nettement apparaître la matière plastique qui devient opaque; on peut alors constater que cette matière est plus abondante autour des gouttelettes huileuses, comme le montre la figure 1 (plancles). Après la ponte, la partic plastique se eoncentre en une lentille biconvexe qui fait saillie au-dessus du vitellus et, qui se forme dans le voisinage du micropyle, comme l'a déerit II. Gerbe.

Chez l'Épinoche on observe les mèmes pliénomènes, mais au moment de la ponte la matière plastique est encore plus ćtalée que clee la Truite et recoure prestue toute la moitic du vitellus, elle se concentre en unr lentille cicatriculaire, 
après la fécondation, par suite de contraction de toute la masse de l'oouf.

Un mode de constitution du genre semblable à celui que j’ai observé chez la Truite a été signalé, en 1864, par M. le professcur Balbiani (6). Dans l'œuf de Grenouille rousse, du Géophile et dans l'ovule de la Femme, il a vu se fomer autour de la vésicule embryogène des globules dans lesquels apparaissent des granulations très fines. Puis fees globules se détachent de la vésicule, se désagrègent à la périphérie de l'œuf el les granulations forment une couche gramuleuse qui constitue le germe. Chez les I'leuronectes, M. Balbiani a constaté aussi que la couche plastique granuleuse se forme également autour de la vésicule embryogène. Dans l'œuf de la Truite, c'est autour de la résicule germinative que se constituentles globules plastiques; mais n'ayant pas fait de reclierches personnelles sur le développement de l'œuf ovarien, je ne m'occuperai pas ici de la genèse de ces éléments.

Les éléments vitellins des Poissons osseux ne se fusionnent pas toujours au monient de la chute de l'œuf pour constituer, comme chez la Truite, une masse vitelline homogène. Hoffmann a vu, en effet, que le vitellus du lfareng conserve son aspect vésiculeux pendant tout le développement de l'œuf. J'ai observé le même fait dans l'œuf du Lepadoguster; le vitellus de cet ouf renferme des vésicules transparentes, de volume variable, de forme polygonale par suite de la pression réciproque quielles cxercent les unes sur les autres, ct contenant souvent de petits globules luileux dans leur intéricur; mais il y a aussi de gros globules luuileur libres au centre du vitellus, sous le disque germinatif.

Jusqu jei je ne me suis pas ocetupé d'un élément important de l'wuf, de la vésicule germinative. C'est qu'en effet, ainsi que je l'ai dit plus haut, cette vésícule n’existe plus dans l’œuf ponclu; mais on la trouve dans l'wuf ovarien et il me reste à étudier son mode de disparition.

De tous les phénomènes quí accompagnent la maturation de l'œuf, il n'en est pas quí ait soullevé plus de discussions que celui de lis disparition de la vésicule germinative, et les oufs des Poissons osseux ont ett lobjut de plusieurs traraus is eet igard. 
Vogt (191) a vu que, chez la Palée (Coregonus palea), dans l'œuf mûr on ne trouve plus aucune trace de la vésicule germinative " quoique, dit-il, on ne puisse douter de son existence jusqu'à la fécondation. "Il pense que la vésicule se maintient dans le disque huileux et que les nombreuses taches germinatives qu'elle contient continuent à se développer après la fécondation et deviennent les premières cellules de l'embryon.

Lereboullet (111), chez le Brochet, a constaté aussi la disparition hâtive de la vésicule germinative, disparition qui, d'après lui, aurait lieu avant que l'ouf ait atteint sa maturité et ses dimensions ordinaires; il dit avoir cherché en vain la vésicule sur plus de cent wufs, mesurant de 1 millimètre à $1^{\mathrm{mm}, 5}$, et provenant de divers individus; mais il a constamment trouvé près de la surface de l'œuf un ou plusieurs anas irréguliers, devenant blanchâtres, par la coagulation, comme la vésicule clle-mème dans les jeunes ovules. Il pense que ces amas sont les débris de la vésicule germinative. "A mesure que l'œuf approche de la maturité, les débris de la vésicule germinative se dispersent sous la forme des flocons jaunatres dans toute l'étendue de l'œuf et se mèlent aux éléments du vitellus. "

Les observations de Lereboullet (112) sur les œufs de la Truite sont intéressantes à rappeler, car j’ai pu en vérifier en grande partie l'exactitude. Dans les œufs mûrs ou presque mûrs, cet auteur a vu la vésicule germinative vidée et ratatinée, tout à fiit à la surface. Sur des œufs coagulés, il a trouvé à la surface un petit disque blanc, très apparent, visible à travers le chorion et qu'on pouvait facilement détacher avec des aiguilles. "Le disque mesure $0^{\mathrm{mm}}, 6$. En le déchirant on voit qu il constitue un véritable sac vide, aplati, chiffonné; cependant il contient encore assez souvent des amas de corpuscules celluliformes, gramuleux, dont le diamètre varie de $0^{\mathrm{mm}}, 008$ à $0^{\mathrm{mm}}, 16$ et dont quelques-uns sont nucléés. "Dans les œufs pondus, Lereboullet n’a trouvé dans le vitellus que quelques lambeaux jaunàtres, composés de vésicules brillantes et qu’il croit provenir des débris de la vésicule germinative.

OEllacher (122), en 1872, a décrit la disparition de la vésicule germinative chez la Truite, et il a voulu généraliser les phénomènes qu'il avait observés, en admettant un mème mode de disparition chez tous les Vertébrés. Suivant lui, plus ou moins 
lôt avant la fécondation el quelquefois aļrès. la vésicule germinative, qui s'est rapprochée de la périphérie de l'œul' pendant lit maturation, arrive all milien du germe, au-dessous du micropyle. Lat vesicule sompre alors comme une bourse et sobtale de telle sorte que sal membrane forme une espece de voile à lit surlace du germe: ce voile présente des stries très funes perpendiculaires it sa surface; quant au contemu do la vésicule, il séchlppe comme une masse floconneuse. Les tiches germinatives restent encliassées dans l'épaisseur du voile.

Pour les Poissons cautiligineux, Schenk(167) a vu chez la liaie, la vésicule germinative disparâtre dans la cicatricule et ì sil place il a observé une cavité communiquant avec la surfice palr un petil orifice. Balfour (8) a observé de son côté ì la surlitec dı germe de la Reaja balys une lache claire formée par la membrane plissece de la visieule germinative: il croit que la unembrane est expulsée el que le contour cst absorbé sur place par le germe.

Plus rócemmont, Calberla (30) a supposé que clez le pelromyson la vésicule germinative disparaissait de très bonne heure laus l'oull ovarien, an moment de la transformation de l'Ammucète en Lamproie, mais Scott (172) a réfuté celle rrrunr et a trouré que la vésicule zerminalive persiste jusqua au moment dri lia ponte.

Sillensky (163) a constaté que la vésioule germinalive de l'ouf du Sterlet disparaissait dams les premiemes hemes qui smivent la ponte. A sa place on aperegoit dims le germe plusieurs petits iluts formés par une substance transpanenule et qui sont dispersés dims la masse du germe; par leurstruclure, ils ressemblent à la vésicule germinative parlate. Les modifications suceessives de relte dernière sont analogues a celles que fol (50) a signaleses cliez les Astérirs.

Tel chail lialat de la question lorsque jentrepris mes re-

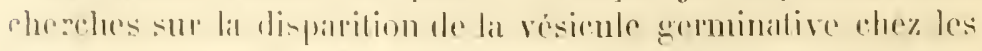

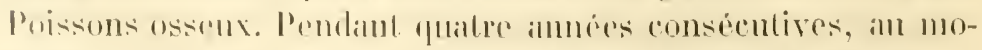

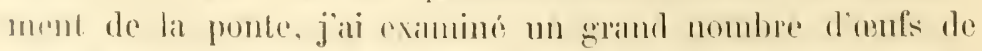

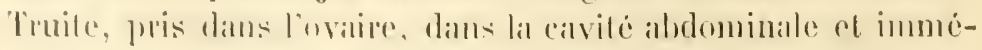

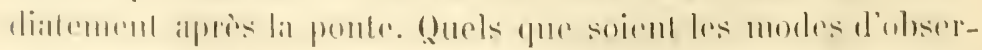

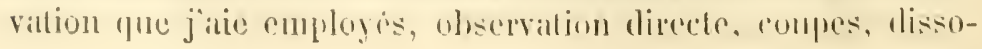


ciation, quels que soient les réactils dont je me sois servi, je n’ai pu élucider cet intéressant problème. Je suis parvenu cependaut a me convainere que la plupart des descriptions données par nues prédécesseurs, et entre autres celle d'OEllacher, sont entachées d'erreur.

Dans tous les œufs ovariens que jai examinés au moment de la ponte, jai trouvé la vésicule germinative. Sur les œufs frais elle apparait comme une tache claire, arrondie, mesurant de $0^{\mathrm{mm}}, 48$ à $0^{\mathrm{mm}}, 冖 3$ et située dans le voisinage lu micropyle. (Planclies, fig. 1,vg.) Dans son intéricur on voit les taches germinatives nombreuses, réunies en un petit amas an centre. Si l'on traite l'œul' par l'acide acétique, la vésicule devient opaque et beaucoup plus nettement visible. Des coupes pratiquées à travers les cufs ovariens, durcis par l'alcool ou l'acide chromique, montrent la vésicule gerninative sous forme d'un corps lenticulaire situé à peu de distance de la surface de l'ceuf et entouré des vésicules granuleuses que jaai décrites à propos de la formation du germe. (Planches, fig. 3,vg.) La membrane de la vésicule est encore bien nette et présente un double contour; au ecntre se trouvent les tiches germinatives qui ont quitté la portion périphérique qu'clles occupaient primitivenent pour se rémir en un pelil amas. Le reste de la vésicule est rempli par une substance tres finement granuleuse, résultant de la coagulation du liquide qu'elle renfermait; dans cette substance on observe quelques gramulations plus grosses et plus réfringentes, disséninées irrégulièrement.

Une disposition tonte semblable s'observe chez la Grenonille, dans l'œuf ovarien mìr avint la rupture du follicule, ainsi que Ilertwig (78) l'a signalé. Seulement, ehez les Batraciens, la paroi de la vésicule germinative est plissée, tandis que celle des Poissons reste tendue; les taches germinatives sont réunies aussi au centre de la vésicule.

Chez l'binoche et l'ipinochette, la vésicule germinative présente le même aspect glue chez la Truite el se voit nettement all-dessous du mieropyle, dans les aufs ovariens.

Je n'ai pu malheureusement assister it la disparition de la vésicule germinative; malgré les nombreuses pecherches que jai faites sur des ands de Truite, an moment du lrai, je n'ai jamais pu surprendere lo monent précis où eetle vésicule, si 
visible dans l'œuf ovarien, se transforme probablement en noyau de l'œuf. Mes recherehes, infructueuses relativement au but que je me proposais, m'ont cependant convaincu que la description donnée par OEllacher (122) repose sur une erreur d'observation.

J'ai toujours trouvé la vésicule germinative dans tous les œufs ovariens que jai examinés, même dans ceux dont le follicule commençait ì se rompre: cette vésicule se présentait avec les caractères que j'ai décrits plus haut. Dès que l'œuf est tombé dans la cavité abdominale, on ne trouve plus trace de la résicule; la constitution de l'œuf a subi alors une transformation complète, ainsi que je l'ai déjà dit. C'est donc pendant le temps très court que l'œuf met à être expulsé du follicule ovarien que disparait la vésicule germinative. Or, OEllacher prétend avoir observé la vésicule germinative s'ouvrir et s'étaler ì la surface du germe dans les oufs pris dans la cavité alodominale, dans les œufs pondus et même fécondés. Cette simple affirmation suffit à démontrer qu'OEllacher a pris pour la vésicule germinative un aspect particulier qui s'observe quelquefois dans le germe. Il arrive, en effet, que sous l'influence des réactifs durcissants, de l'acide chromique principalement, le germe soit soumis à une certaine pression entre la capsule de l'œuf et la masse vitelline. De petites portions du vitellus nutritif traversent alors le germe et viennent s'étaler plus ou moins à sa surface, on former de petites taches qui pourraient être prises pour des taches germinatives. De semblables dispositions ne se voient que rarement dans les œufs traités par le liquide picroacétique.

Plus heureux que moi, C. K. Hoffmann (89) a pu récemment, sur certains ooufs très transparents de Poissons ossenx marins, suivre quelques-unes des phases de la disparition de la vésicule germinative. Dans les œufs nuûrs de Scorprena et de Julis, la vésicule germinative est toujours située contre la capsule, au-dessous du micropyle; les taches germinatives disparaissent et la vésicule ne renferme plus alors qu'un liquide dans lequel se sont dissous les nucléoles et le réseau. Bientôt la membrane d'enveloppe de la vésicule disparaît et son contenu se mêle à celui de l’œuf. A la place occupéc par la vésicule, apparait un fuscan de direction semblable à celui que 
Fol (50) et Hertwig (78) ont vu se former dans l'œuf des Echinodermes, au moment de la disparition de la vésicule germinative. Le fuseau de direction mesure chez le Scorpena $0^{\mathrm{mm}}, 025$ de long sur $0^{\mathrm{mm}}, 0143$ de large; son grand axe forme avec celui de l'œuf un angle de $45^{\circ}$, et l'une de ses extrémités se trouve à l'ouverture interne du micropyle. Dans l'œuf des Julis, le fuseau est moins net, et lans ceux des Crenilabrus, Heliasis, Gobius, Blemnius, Belone et du Hareng, Hoffmann n’a pu l'apercevoir. Le fuscau de direction donne naissance au pronmcleus femelle et à un globule polaire; celui-ci ne se produirait qu'après la pénétration du spermatozoïde dans l'œuf. Hoffmann a constaté, comme moi, un changement brusque de structure an moment de la disparition de la vésicule germinative; le germe se concentre au-dessous du micropyle, le vitellus de nutrition devient homogène et les éléments huileux se séparent.

Deux auteurs américains, Kingsley et Conn (93) ont étudié aussi le déreloppement les œufs de quelques Poissons marins, entre autres du Ctenolabrus coruleus. Ils ont vu qu'au moment de la maturité de l'œuf, la vésicule germinative est alors invisible et que les globules vitellins disparaissent; l'œuf est alor's transparent et le germe se concentre à l'un des pôles. Dans le disque germinatif, ils ont constaté la présence d'un aster simple et quelque temps après ils ont vu s'échapper de l'œuf par le micropyle, un petit corps qu'ils considèrent comme un globule polaire. Liobservation de ees auteurs vient done confirmer celles de Hoffmann.

Les faits observés par Hoffmann, Kingsley et Conn relativement à la dispariition de la vésicule germinative chez certains Poissons osseux sont tout à fait en accord arec ceux qui ont été vus par un grand nombre d'auteurs chez beaucoup d'Invertébrés, par Büischli (29) chez les Mollusques, Fol (50) et Hertwig (78) chez Ies Echinorlermes, etc. Les observations incomplètes d'Ed. Van Beneden (19) sur l'œuf du Lapin permettent de supposer que elıez les Mammifères la vésicule germinative donne aussi naissance aux globules polaires et au noyau de l'œuf. Pien qu'on n’ait encore rien observé de semblable shez les autres Vurtébres, il y a tont lieu de croire que la disparition de la vésicule germinative suit un processus: 
identique chez ces animaux. Chez la Lamproie, liupfer et Benecke (109) ont vu se produire à la surface du vitellus un globule polaire avant la fécondation et un autre après la fécondation; chez le nême animal, Calberla (30) a constaté la présence d'un pronucleus femelle à l'endroit où avait disparu la vésicule germinative. D’après Salensky (163), la vésicule germinative de l'œuf du Sterlet disparait dans les premières heures qui suivent la ponte. A sa place on aperçoit, dans le garme, un petit corps arrondi qui serait le pronucleus fenelle. En même temps apparait au pòle actif de l'œuf, à la surlace du vitellus, une substance transparente, homogène, analogne au voile que Hertwig (78) a signalé à la surface de l'œuf de la Grenonille. Le corps véliforme est peut-être l'homologue des globules polaires.

La production de globules polaires na jamais été constatée chez les Plagiostomes, les Reptiles et les Oiseaux; mais on ne saurait, ce me semble, en conclure que la vesicule germinative ne se transforme pas chez ces animaux en noyau de l'œuf et en globules polaires. Il se peut très bien, nn effet, que les globules polaires an lieu de se séparer de l'œuf restent inelus dans lo germe et échappent à l'observation. C'est ainsi que, dans l'œuf du Moina rectiroslris, Grobben (61) a vu une masse polaire se différencier au pòle actif de l'œuf, mais rester enclavée dans le vitellus. Ir fait suivant que jai observé chez la firnouille viendrait à lappui de cette mamière de voir. Comme je l'ai déjù dit, la vésicule germinative disparait chez la Grenouille de mème que chez la Truite, pendant la chute de l'œuf lans la cavité abdominale. Dans un point très voisin de la périphérie do l'œul', à l'endroit où a disparu la vésicule germinative, on observe sur des coupes, un espace elair, entouré de jigment, an centre duquel est un petit corps rond, légèrement granuleux, il bords très nets. Par ses dimensions et son aspect. (6) corps resemble tont à fait au noyau femelle que Hertwig 78) al ru dans le voisinage du noyau spermatique. à l'extrémité le l'une des trainées pigmentaires découvertes dans l'œuf des liatracions par Van Bambeke (12). Hertwig a très bien décrit et figuré la fusion de ce zovau femelle aver le noyau màle pour constituer le premier noyau de segmentation. Mais il n avait pu constater lorerigine du noyau femelle. Souvent on apergeit aupres du 
noyau femelle, dans l'espace clair, provenant de la disparition de la vésicule germinative, un ou deux autres pelits corps réfringents, plus petits que le noyau, moins bien délimités et plus rapprochés de la surface de l'œuf. Ces corpuscules me paraissent être des globules polaires qui restent ainsi dans l'œuf et qui sont plus tard résorbés ou expulsés. J'ai pu voir, en effet, quelquefois sur des oufs fécondés de Rana temporaria, an moment de lat lomation du premier sillon de segnentation, un ou deux petits globules de protoplasma incolore. situés au-dessous de la membrane vitelline, dans le sillon de segmentation. Ine observalion analogue a été faite depuis par II. Mathias Duval (43) dans les mêmes conditions. Cet auteur consillère aussi ces globules comme des globules polaties. II se peut que ces corpuseules ne soient expulsés de l'oul' qu'exceptiomellement et deviennent alors visibles, tandis que normalement ils restent inclus dans le vitellus. Il en serait de même chez les Poissons; chez certains d'entre eux, tels que ceux qui ont été observés par Hoflmann, Kingsley et Conn, les globules polaires seraient expulsés de l'œuf. Chez d'autres, tols que les Salmonides, ils demeureraient dans le germie. Si celle lypothèse était confirmée, elle prouverait que les globules polaires n'ont pas une importance aussi grande que le veulent certains auteurs tels que Sedwig-Winot (173), Balfour (9), Sabatier (162) et Ed. Van Beneden (20) qui considèrent ces corps comme une partic màle expulsée de l'œuf, originairement màle et femelle. Que deviendrait cette substance mâle dans le cas où l's globules polaires persistent dans l'œuf et sont résorbés? Récemment 0 . Schultze (171) a observé la formation d'un fuseau de direction et des globules polaires chez les Amphibiens. Ses recherches ont besoin d'ètre reprises afin d'établir si le fait qu il a constaté est général.

L'œuf pondu et non fécondé présente chez beaucoup de l'oissons osseux des modifications qui ont été très bien étudiées par plusieurs observateurs, entre autres par Van Bambeke (11). Cet ouf est le siège de mouvements actifs du germe accompagnés de changements de forme pouvant simuler les premières phases de la segmentation. Dans l'œuf de la Tanche, d'après l'auteur belge, le germe coiffe le vitellus comme une calotte qui prend bientôt la forme dune lentille biconvexe 
Les éléments vitellins, globules huileux et globules albuminoïdes, se rassemblent au-dessous du germe; celui-ci émet des prolongements filiformes, semblables aux pseudopodes d'un Rhizopode, qui vont pour ainsi dire pêcher les éléments vitellins pour les accumuler au-dessous du germe. Le noyau vitellin ainsi constitué est formé d'une masse centrale finement granuleuse, entourée d'éléments plus volumineux. Le germe, doué en même temps de mouvements amiboïdes énergiques, change constamment de forme. Suivant Van Bambelie, ces mouvements auraient pour but de mettre les différents points du protoplasma plastique en contact avec les éléments nutritifs. Le noyau vitellin peut être, en effet, momentanément emprisonné en partie par le germe. Le disque germinatif présente des protubérances qui simulent un commencement de segmentation; il se détache quelquefois de sa masse de petites portions quon pourrait prendre pour des globules polaires. Les mouvements amiboïdes persistent quatre heures après la ponte. Chez la Lote, les changements de forme du germe sont beaucoup plus lents et moins prononcés. De semblables mouvements du germe, accompagnés de l'accumulation des éléments huileux du vitellus au-dessous du disque germinatif, ont été signalés chez d'autres Poissons osseux : chez le Brochet, par Lereboullet (111), Reichert (143-144), His (82) et Ramson (134); chez la Perche, par Lereboullet (111); chez l'Épinoche, par Ramson (134); enfin chez les Salmonides, Truite, Saumon et Ombre, par Stricker (186), Kupffer (104), IVeil (195), OEllacher (123) et His (82).

Dans les œufs assez volumineux, à capsule épaisse et peu transparente, des Salmonides, la constatation des mouvements du germe est diffieile: elle no peut se faire que d'une manière indirecte, en durcissant rapidement les œufs, comme l'ont fait Stricker et ONllacher, et en observant à la surface du germe des bosselures, et sur ses bords des irrégularités. En me placant dans ces conditions, jai pu vérifier les observations de ces auteurs.

Lorsqu'on met dans l'eau un œuf de Truite pondu et non fécondé, on constate au hout de quelques heures que le germe qui était primitivement étalé à la surface de l’œul', ainsi que je l'ii dit, s'est coneentré et est devenu beaucoup phus visible. 
Dans son mourement de contraction, il entraine avec lui un grand nombre de gros globules huileux qui se trouvaient dans la couche corticale. Le germe ainsi rassemblé devient alors très apparent, c'est ce qui avait fait croire à Coste qu'il ne se formait qu'après la ponte. Chez l'Épinoche on peut suivre par transparence la coneentration du germe. Les mouvements qui s'observent alors sont analogues à ceux qui se voient dans l'œuf après la fécondation, et que je décrirai plus tard.

\section{II. - Fécondation.}

La fécondation chez les Poissons osseux, sauf chez quelques rares espèces ovovivipares, telles que le Zoarces viviparus, est externe; elle s'opère dans l'eau, le mâle émettant sa semence sur les œufs pondus par la femelle. Aussi la fécondation artificielle est-elle très facile chez ces animaux; pratiquée pour la première fois par Jacobi (1), vers le milieu du dernier siècle, cette opération est devenue tout à fait classique depuis les belles recherches de Coste.

Les spermatozoïdes de Salmonides sont complètement immobiles dans la laitance extraite de l'animal. Dès qu'il sont mis en contact avec l'ean, ils exécutent des monvements de trépidation très vifs qui ne durent que quelques instants, puis ils redeviennent immobiles; la durée de ce mouvement ne dépasse pas trente secondes. Coste admet que la vitalité des spermatozolldes persiste pendant sept ou huit minutes, parce qu'il a pu au bout de ce temps féconder des œufs avec de l'eau spermatisée. Ce résultat tient à ce que la laitance forme une masse assez compacte qui ne se mêle à l'eau que peu à peu, de sorte que les spermatozoïdes du eentre de la masse ne se

(1) “Vers le milieu du dernier sieele, en 1758, le eomte de Goldstein, grand chancelier des duchés de Bergues et de Juliers pour son Altesse palatine, remit à l'un des ancêtres du célèbre Foureroy un mémoire sur la fécındation artificielle des œufs de poisson el sur l'emploi de ce procédé pour le repeuplement des rivières et des ètangs. Ce remarquable travail qu'il tenait d'une personne en qui il avait la plus grande confiance, et dont Jacobi était l'auteur, ètait écrit en allemand, et M. de Fourcroy, trouvant des difficultés à le traduire, le comte de Goldstein voulut bien le lui donner en latin. La version francaise fut publiée en entier, en 1773, dans le Traité général des péches de Duhamel du Moneeau, réligé par ordre de l'teadémie des sciences. " Coste, Instructions pratiques sur la pisciculturc. Paris, 18b3, p. 1. 
mettent en mouvement que lorsque les autres sont morts depuis déjà longtemps. On peut constater ce fait en mettant un peu de laitance au contact de l'eau sous le champ du microscope; on voit alors les spermatozoïdes se désagréger petit à petit autour de la goutte de laitance, et s'éloigner en tourbillonnant dans l'eau; il s'écoule plusieurs minutes avant que toute la laitance soit mêlée à l'eau, et pendant tout ce temps on voit des spermatozoïdes mobiles, et d'autres qui sont morts depuis longtemps. Hais si l'on mélange rapidement la laitance à l'eau, de manière à mettre tous les spermatozoïdes à peu près au même moment en contact avec le liquide, on constate que les mouvements ne persistent que quelques secondes.

Chez l'Épinoche et l'Épinochette, la vitalité des spermatozoïdes est beaucoup plus grande que chez les Salmonides. En pratiquant des fécondations artificielles d'Épinochette, j’ai vu les spermatozoïdes tournoyer dans l'orifice micropylaire des œufs pendant vingt à trente minutes; quelques-uns même étaient encore mobiles au bout d'une heure et demie. C.-K. Hoffmann (89) a constaté aussi que plusieurs Poissons de mer, pour lesquels il a pu pratiquer la fécondation artificielle, Scorpona scrofa, S. porcus, Julis vulgaris, Crenilabrus pavo et Heliasis chromis ont des spermatozoïdes doués d'une assez grande vitalité. Toutefois ils perdent leurs mouvements après un séjour de trente minutes dans l'eau de mer.

Dans une note présentée à l'Académie des sciences, en 1877 , j'ai fait connaitre les résultats de mes recherches sur la vitalité des spermatozoïdes de la Truite et sur la fécondation des œufs avec de la laitance mélangée à certains liquides $(\mathbf{6 7})$.

Sur une centaine d'œufs de Truite fécondés avec de la laitance mêlée à l'eau depuis une minute, aucun œuf ne s'est développé. Après quinze secondes de contact avec l'eau, les mouvements des spermatozoïdes sont lents et peu étendus; un grand nombre mème sont déjà immobiles; soixante øufs fécondés avec ce sperme affaibli ont donné quarante-six éclosions. Le sperme peut, au contraire, garder longtemps sa vitalité lorsqu'on le conserve à sec dans un milieu humide. Ainsi de la laitance laissée pendant quatre jours dans un flacon bouché, à une température de 10 à 1 ỏ degrés, a pu servir à féconder quarante ceufs, sur lesquels huit seulement ne se sont 
pas développés; j’ai même pu féconder des œuf́s avec le la laitance datant de six jours.

J'ai pratiqué une série de fécondations avec de la laitance soumise à l'action de l'alcool et des anesthésiques. Des œufs provenant d'une même ponte furent essuyés avec soin sur du papier à filtrer, afin de les débarrasser du liquide péritonéal qui pouvait y adhérer, et empêcher ainsi les mouvements des spermatozoïdes. On mit sur un certain nombre de ces œufs quelques gouttes de sperme, puis on versa sur le tout de l'eau alcoolisée à $\frac{5}{100}$; au bout de quelques minutes, les oufs furent placés dans l'eau courante. On traita de même les autres œufs en les arrosant d'abord avec de la laitance fraîche, puis avec de l'eau alcoolisée à $\frac{10}{100}$, de l'eau éthérée, de l'eau chloroformée et de l'eau pure. Voici quels furent les résultats de ces fécondations :

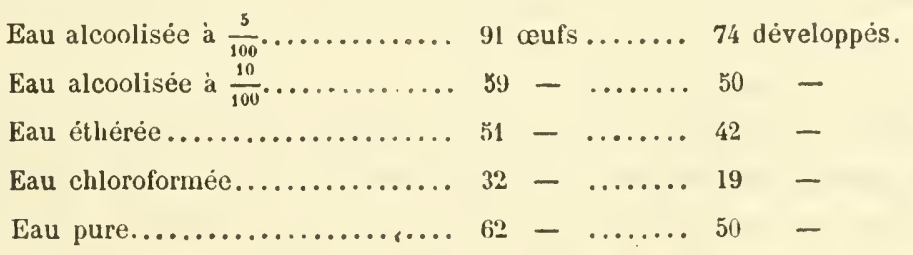

Les éclosions ont eu lieu toutes à la même époque, et les petites Truites provenant de ces différents œufs n'ont présenté dans la suite aucune particularité qui pût les faire distinguer des alevins obtenus par fécondation normale.

Ces expériences prouvent que l'alcool et les anesthésiques n'exercent pas une action nuisible sur les spermatozoïdes de la Truite, à des doses suffisantes pour tuer cependant des animaux inférieurs, tels que des Infusoires. Cette innocuité n'est probablement qu'apparente; la vitalité des spermatozoïdes des Salmonides a une durée si courte que l'alcool et les anesthésiques n'ont pas le temps d'agir sur ces organismes avant leur pénétration dans l'œuf; dès qu'ils sont entrés dans le germe, ils sont à l'abri des substances toxiques. Il est probable que les spermatozoïdes d'autres Poissons, comme ceux de l'Épinoche qui présentent une vitalité beaucoup plus longue, se comporteraient différemment mis en présence de l'alcool et des anesthésiques, et perdraient leurs mouvements plus tôt que dans l'eau 
pure. Je n'ai pu malheureusement faire des recherches à ce sujet.

Les œufs mûrs de la Truite, mis dans l'eau sans être fécondés, perdent aussi assez rapidement la propriété d'être fécondés ; je n'ai pas déterminé exactement la durée de la période pendant laquelle les œufs restent fécondables; elle ne dépasse pas très probablement une demi-heure. L'œuf absorbe en effet de l'eau qui gonfle la capsule, et le micropyle se trouve ainsi fermé. Les œufs pondus, conservés à sec dans une atmosphère humide et à une température ne dépassant pas $12^{\circ} \mathrm{C}$., conservent leur propriété d'être fécondés pendant deux ou trois jours. Des œufs datant de deux jours fécondés avec du sperme frais ont donné trente-deux éclosions sur quarante œufs; des œufs datant de quatre jours ont donné cinq éclosions sur vingt œufs, et des œufs datant de cinq jours, une seule éclosion sur vingt-deux œufs. L'œuf extrait de l'animal perd done sa vitalité plus vite que ne le fait le spermatozoïde.

Pour les œufs qui ont un petit volume, comme ceux de l'Épinoche, il est facile de suivre directement, sous le microscope, les premières phases de la fécondation, e'est-à-dire l'entrée des spermatozoïdes par le micropyle. Pour les œufs des Salmonides, l'observation est beaucoup plus difficile. Cependant en faisant tomber, immédiatement après la fécondation, des œufs dans une solution d'acide chromique, assez forte, afin de les tuer rapidement, et en pratiquant ensuite des coupes à travers la région micropylaire, on peut voir des spermatozoïdes engagés dans le canal du micropyle. Mais, lorsqu'on vent retrouver les éléments mâles dans l'intérieur de l'œuf, on éprouve une difficulté insurmontable, Le spermatozoïde est tellement petit par rapport à l'œuf qu'il est impossible de le distinguer après sa pénétration dans la masse granuleuse du disque germinatif. J'ai essayé un grand nombre de fois de rechercher le ou les spermatozoïdes dans le germe de la Truite et de l'Épinoche, soit sur des coupes d'œufs durcis immédiatement après l'imprégnation, soit dans des germes extraits de l'œuf vivant. Les réactifs colorants, tels que le vert de méthyle, le carmin aluné, l'hématoxyline, qui ont une grande affinité pour la substance chromatique constituant la tête du spermatozoïle, colorent aussi la totalité du germe, de sorte qu ils ne 
peureml servir pour de semblables recherches. De julus, le germe renferme un grand nombre de corpuscules refringents qui ont tout à fait les dimensions et l'aspect d'une tête de spermatozoïde.

Chez l'Épinoche et l'Épinochette, grâce à la transpitrence de l'œuf, j’ai pu souvent observer les modifications qui suivent l'imprégnation. Quelques minutes après l'entrée des spermatozoïdes daus l'œuf, on voit apparaître, entre la capsule et le vitellus, un espace clair, dû à la rétraction du vitellus et à l'entrée d'une cerlaine quantité d'eau, ainsi que Miescher l'a démontré pour les Salmonides. Le germe de l'Épinoche est, comme je l'ai déjà dit, trés mal délimité et très étendu dans l'œuf pondu. La couche corticale granuleuse et peu transparente, aux dépens de laquelle se constitue le germe, paraît avoir à peu près la mème épaisseur sur tout le pourtour de l'œuf. Lorsque commence la rétraction du vitellus, la couche corticale s'épaissit sur certains points et forme des bosselures qui donnent au contour du vitellus un aspect irrégulier. Les bosselures cliangent de forme et de place; on les voit parcourir la surface du vitellus comme des vagues qui se déplaceraient très lentement à la surface de l'eau. Toute la masse vitelline est animée des mêmes mouvements et on la voit s’étrangler de distance en distance. Les protubérances, formées par la substance finement granuleuse de la couche corticale, deviennent de moins en moins nombreuses et se fusionnent it l'un des pôles de l'œuf. Elles constituent finalement une seule masse en forme de calotte, renflée en son milieu, et amincie sur ses bords, qui coiffe environ le quart du globe vitellin. (Planches, fig. 6.) Cette masse est encore animée pendant quelques temps de mouvements d'ondulation qui déplacent sa partie renflée, et achèvent sa concentration. Une heure et demie environ après la fécondation, le germe de l'œuf d'Épinoche est définitivement constitué et a pris la forme d'une lentille planconvexe, qui repose par sa face plane sur le vitellus, et se continue par ses bords amincis avee la couche corticale entourant le globe vitellin. Pendant les mouvements de concentration du germe, les éléments huileux, qui étaient disséminés à la périphérie de l'œuf, dans la couche corticale, se sont rassemblés an-dessous du disque germinatif, auquel ils sont quelquefois reliés par des filaments protoplasmiques analogues it 
ceux que Van Bambeke (11) a décrits dans l'œul de de la Tanche.

Ramson(134), qui a étudié, clıez l'Épinoche, les mouvements que je viens de décrire, pense qu'ils sont dus à une contraction de la couche plasmique de l'œuf. His (82) admet aussi que les mouvements très curieux qui s'observent dans l'œuf du Brochet, se passent dans la couche corticale. Je partage entièrement cette opinion. Le vitellus est constitué, en effet, par une masse albumineuse spéciale qui ne saurait ètre douée de contractilité, tandis que li couche corticale possède toutes les propriétés du protoplasma et entre autres la contractilité.

Il est probable que dans l'œuf des Salmonides, il se passe des phénomènes semblables à ceux dont l'œuf de l'Epinoche est le siège, mais l'opacité de la capsule empèche de bien les observer. Stricker (186) et OEllacher(123) en durcissant des œufs de T'ruite, pris à des moments de plus en plus éloignés de leur fécondation, ont constaté, après avoir enlevé la capsule, des changements de forme dans le germe, se traduisant par des bosselures. De semblables bosselures irrégulières s'observent, en effet, à la surface du germe, quelques heures après la fécondation, mais je les ai trouvées beaucoup moins prononcées que ne les ont figurées Stricker et OEllacher; cela tient, je crois, à ce que je tuais les culs plus rapidement que ces deux auteurs, de sorte qu'il ne se produisait pas de contractions irrégulières du germe sous l'influence des réactifs.

Lorsqu'on pratique des coupes à travers des œufs durcis dans les premières heures qui suivent la fécondation, on peut suivre le phénomène de la concentration du germe et voir comment il devient de plus en plus épais, et finalement une lentille plan-convexe. Le germe se présente d'abord sur une coupe médiane, une demi-heure après la fécondation, comme un croissant très mince, qui recouvre à peu près le sixième de la circonférence du vitellus. Une heure et demie à deux heures après, lo croissant a diminué d'étendue, et sa partie médiane s'est renflée en forme de lentille biconvexe, dont les bords se continuent avec ceux du croissant. Ce n’est que six à huit heures après la fécondation que le germe a achevé sa concentration; il lui faut souvent un temps beaucoup plus considérable, lorsque la température est basse. Il se présente alors sous la forme d'une masse lenticulaire à bords épais dont la face 
supéricure libre est convexe, et la face inférieure, mal délimitée par rapport au vitellus sous-jacent, est plus aplatie.

Le germe est constitué par une substance finement granuleuse, au milieu de laquelle se trouvent de petits éléments arrondis, réfringents, homogènes, de nature protoplasmique, et qui sont de plus en plus volumineux de la surface vers la profondeur. Ces éléments se retrourent dans la couche corticale qui s'étend au-dessous du germe à la surface du vitellus, et qui constitue plus tard le parablaste. On trouve parfois à la surface dı germe de petits corps lenticulaires d'une coloration jaunâtre ou brunâtre, d'une réfringence plus grande que celle de la substance germinative et qui semblent enchâssés dans la couche superficielle. Ce sont les petites globules vitellins, dont j’ai parlé et qu'OEllacher a pris probablement pour les taches germinatives.

J'ai cherché avec soin sur de nombreuses coupes de germes, l'existence des noyaux mâle et femelle et je n'ai jamais pu les rencontrer. Malgré cela, je suis tout disposé à admettre qu'ils existent chez les Salmonides et qu'ils se comportent comme Hoffmann (89) l'a constaté chez d'autres l'oissons. Cet habile observateur a vu, en effet, que chez les Poissons marins, chez lesquels il a suivi la transformation de la.vésicule germinative en noyau femelle et en globules polaires, le spermatozoïde, après son entrée dans le germe, se transforme en un noyau mâle entouré d'un aster. Le noyau mâle et le noyau femelle grossisssent en s'approchant l'un de l'autre el se fusionnent pour former le premier noyau de segmentation.

Dans plusieurs germes de Truite encore largement étalés sur le vitellus, jai observé une disposition rayonnante du protoplama autour d'un centre situé sur la ligne médiane du germe; mais au centre de ce grand aster, dont les rayons atteignent presque la périphérie du disque germinatif, je n'ai pu trouver, avec le plus fort grossissement, ni à l'aide des réactifs colorants, aucune trace de noyau. A la fin de la concentration du germe, quelque temps avant la segmentation, on voit aussi au centre du germe un aster à rayons très étendus, entourant un espace clair qui renferme un noyau à contours mal délimités; ce noyau est évidemment le premier noyau de segmentation. Waldner (194) n'a pu ennstater anssi la présenee du premier 
noyau de segmentation chez la Truite que sept heures après la fécondation : le noyau était situé au milieu du germe, plus rapproché de la surface externe que de la surface interne.

Kupffer (108) qui a étudié récemment la fécondation de l'œuf de Truite, décrit les phénomènes suivants : dans l'œuf non fécondé, sur une coupe pratiquée à travers la région du micropyle, on distingue un petit disque central avec un corpuscule excentrique, solide, se colorant en jaune par le picrocarmin, et une zone externe radiéc. Dix minutes après la fécondation, on observe à la surface du germe une membrane délicate, qui vingt minutes plus tard se montre striée perpendiculairement et présente, au milieu d'un bouchon de substance germinative, des disques polaires. Entre le germe et le micropyle, Kupfer a vu de petits corps aplatis, formés d'une substance finement granuleuse, dans lesquels il aurait trouvé deux fois, un noyau colorable, ce sont les cellules polaires. Une heure après lia fécondation apparait, au sommet apical du germe, une figure allongée, composéc de quatre petites vésicules et entourée d'une zone elaire, perpendiculaire à l’axe de l'œuf. L'auteur n’a pu déterminer si cette sorte de pronucleus était mâle ou femelle. Deux heures et quart après la lécondation, on observe une autre figure semblable, situéc plus profondément et parallèle à l'axe de l'œuf. Trois heures après la fécondation, le germe présente deux noyaux qui se conjuguent bientôt suivant la direction de l'are de l'œuf. Juscu'ì la dixième heure on ne trouve plus qu'un seul noyau. Enfin, entre la douzième et la dix-huitième heure commence la division du premier noyau de segmentation, Je plan de division coïncidant avec celui de l'axe de l'wuf.

\section{III. - Segmextatiox.}

La segmentation de l'œuf des Poissons osseux, vue pour la première fois en 1836, par Rusconi (152-153) chez la Tanche, a été étudiée et décrite souvent par un grand nombre d'auteurs, de Filippi (46), Vogt (191), Aubert (3), Lereboullet (111-112), hupffer (104), Stricker (186), OEllacher (123), Owsjamikow (124), Klein (94), His (83), Van Bambeke (11), Van Beneden (1\%), C. K. Holfmimn (89), lïngslèy et Conm (95), 
Ziegler (200), Agassiz et Whitman (1), Rycler (154), Brook (25-26), ron Kowalewski (103), List (117) et Fusari (52). Aussi ne m'attarderai-je pas à la décrire avec détail, ni à réfuter certaines erreurs d'observation, telles que celle de Striclier qui croyait que les sphères de segmentation se produisaient par bourgeonnement, erreur qui a été très bien relevée par OEllacher et par Klein. J'insisterai cependant sur quelques points particuliers que jai éludiés plus spécialement.

La segmentation chez un certain nombre de Poissons, la Perche, l'Epinoche, ete., suit une marche régulière dont le type est le suivant :

Le germe se divise en denx parties égales par un sillon médian. Un second sillon perpendiculaire au premier partage chacune des moitiés en deux autres, de sorte que le germe est divisé en quatre parties. Deux antres sillons parallèles au second partagent le disque germinatif en huit segments; mais la disposition de ces segments sur deux rangs parallèles donne au stade VIII des Poissons un aspect particulier; qui ne se retrouve pas dans l'œuf d'autres animaux. Le stade XVI est obtenu par la formation de deux sillons parallèles au premier.

Chez la Truite on retrouve assez souvent ce type régulier de segmentation; mais on peut aussi distinguer un autre type derivé du premier et se rapprochant plus de celui qui s'observe chez les Batraciens.

Le premier sillon de segmentation, ainsi qu'OEllacher l'a signalé le premier, apparaît généralement sur l'un des bords lu germe sous forme d'une petite dépression linéaire plus accentuée ì quelque distance du hord libre du germe que sur ce bord même. Quelquefois la dépression se produit au centre même du disque et s'étend vers l'un des bords avant de se diriger vers le côté opposé. Le germe, au moment de l'apparition du premier sillon de segmentation, entre la dixième et la vingtquatrième heure après la fécondation, ne présente pas encore un contour bien net, comme il en aura un quelque temps après; il existe à sa périphérie des encoches et des prolongements irréguliers, qui semblent être des expansions protoplasmiques fixées par l'action du réactif durcissant. Après la formation du premier sillon, le germe est généralement circulaire, mais i] présente sbuvent une forme elliptique, le sillon correspondant 
au petit axe de l'ellipse; quelquefois le premier sillon est $c x-$ centrique et divise l'ellipse en deux parties inégales. (Planches, fig. 25,26 et 27.$)$

Sur les bords du premic sillon, ainsi que sur ceux des antres sillons, on observe des plis radiés très fins, identiques à ceux que Max Schultze (1\%0) a si bien décrits et figurés sur les bords des sillons de l'œuf de Grenouille, les plis se voient très bien sur l'œuf rivant de l'Epinoche.

Le deuxième sillon de segmentation est perpendiculaire au premier, et commence vers le centre du germe, soit immédiatement en partant du premier sillon, soit à une petite distance de chaque côté de ce point. (Planches, fig. 30 et 31.) 11 arrive très souvent que, lorsque le deuxième sillon est terminé ou quelquefois pendant sa formation, il s'opère des modifications dans la forme et le rapport des quatre segments. Ceux-ci se séparent à leur angle interne, de sorte que deux segments opposés viennent se mettre en rapport suivant une petite ligne droite et que les deux autres se trouvent séparés. Les axes des sillons se trouvent ainsi modifiés, deviennent brisés et ne sont plus à angle droit.(Planches, fig. 34.) Rauber (142), qui a publié récemment un mémoire intéressant sur la segmentation de l'œuf des Brataciens et des Poissons, a appelé l'attention sur cette disposition des deux premiers sillons. Suivant lui, le deuxième sillon ne se formerait pas ordinairement, chez la Grenouille, suivant le schéma classique établi par Prévost et Dumas (12 $\mathbf{z})$. Deux petites dépressions viendraient aboutir isolément de chaque còté du premier sillon, à une certaine distance l'une de l'autre, de manière à être reliées entre elles par une ligne de brisure dépendant du premier sillon. Cette disposition qui se retrouve chez les Poissons osseux constituerait le type ranoïde de la segmentation de l'œuf de ces animaux.

La figure décrite par Rauber s'observe souvent, en effet, chez la Grenouille, mais elle semble résulter, comme chez la 'Truite, d'un déplacement secondaire. Chez les Poissons osseux elle n’a pas été signalée ni par les anciens auteurs, tels que Rusconi, Lereboullet, Stricker, OEllacher et ni par Ziegler, (qui dans ces derniers temps a suivi le développement du Saumon. Seul C. K. Hoffmann (89) a figuré (pl. Y, fig. 4) dans un wuf de Julis une disposition du stadr IV semblable ì celle 
que jail observée chez la Truite; mais dans le teste de son mémoire il n’appelle pas l'attention sur ce sujet.

Le stade Vill de la Truite résulte de la formation de deux nouveạux sillons parallèles au premirr. (Planches, fig. 37.) Mais souvent, à partil de ce stade, la segmentation présente de grandes irrégularités. Chaque moitié des deur nouveaux sillons se forme isolément et peut affreter des dimensions très variables. Normalement les deux moiliés d'un même sillon riennent aboutir au même point dı sillon diamétral, perpendiculaire, en se plaçant plus ou moins dans le prolongement de l'un de l'autre; mais le plus souvent elaque moitié aboutit séparément au sillon diamétral en convergeant vers le centre ou vers la périplérie du germo. Colui-ci se tronve alors decoupé à sa surface en lunit segments de forme et de volume dissemblables. L'irrégularité de la segmentation peut être encore julus marquée dès le stade Vhll et le germe peut offrir les aspects figurés. (Planches, fig. 3.; 36, 38 el 39.)

Le stade XVI se présente très rarement avec la régularité qui s'observe chez l'E pinoche, et dont la figure 41 (Planclies) donne un exemple. A partir de ce moment, le fractionnement du germe se fail par des bipartitions égales ou inégales do claque segment, et les lignes de division peurent affecter des directions très variables. Certains segments sont quelquefois en retard sur les autres el restent pendant quelque temps plus volumineux; mais finalement toute la surface du germe se trouve découpe en une trentaine de segments qui, primitivement polyédriques, affectent alors une lorme plus ou moins spliérique. (Planches, fig. 4.t et 4.).) La segmentation continue alors ì se laire régulièrement, les cellules derenant de plus en plus petites et de plus en plus régulières.

L'étude de la segmentation sur des coupes, commo l'a l'ail le premier OAllacher (123), est benueouy plus intéressante que le simple examen des oufs en surface. Lorsquon pratique des coupes diun germe de Truite perpendiculaires au jremier sillon de segmentation, on constate ì la partie médiane de la surface du germe une petite dépression ì laquelle fait suite une ligne claire, très fine, qui s'étend rers le vitellus. Suirant OEllachrer et Ziegler, le premier sillon ne s'étendrail pas jusqu'au vitellus, et n'entamerait que les couches superficielles du germe: 
Balfour (8) pour les Plagiostomes, Salensky (163) pour le Sterlet, Sarasin (164) pour les Reptiles, ont signalé le mème fait. Au contraire Coste (33 his), Hacciel (63), Tan Beneden (19). Hoffmann (89), ont figuré et décrit, chez d'antres Poissons osseux, le premier sillon divisant et traversant le germe dans toute son épaisseur. Mes propres observations mont démontré quil en rst de mème chez la Truite.

La ligne très fine qui fait suite à la dépression superficielle du prenier sillon peut être suivie jusqu au contact du vitcllus, du moins sur les coupes qui passent exactement par le centre du germe. Cette ligne est bordée de claque côté par une petite bande claire nettement différenciée dı protoplasma voisin. A laide d'un grossissement assez fort on constate que ces bandes claires sont traversies par des lignes très fines, parallèles entre elles et perpendiculaires à la ligne médiame; les lignes radiées se perdent dans le protoplasma des deux segments et semblent converger vers les deux preniers noyaux de segmentation. Cette disposition rappelle celle qui s'observe dans les cellules végétales, au moment où se lorme la plaque cellulaire quand se constitue la cloison de séparation des deux cellules filles. (Planches, fig. 60.)

Les premiers segments du germe possèdent-ils une memlirane d'enveloppe? de crois pouvoir répondre à ectle question parl l'affirmative. Il arrive, en effet, quelquefois comme le montre la figure 60 (Planches), qüil se détache à la périphérie du segment des lambeaux très minces qui ont tout à fait l'appalrence diune membrane de cellule. Cette membrane se continue avec lab ligne qui indique la séparation des deux segments; celle-ci me semble done être une cloison se formant aux défens d'une figure radiée analogue à celle des cellules végétales.

(Ollacher (123) a signalé le premier l'existence d’une bande claire indiquant la formation des sillons de segmentation, mais il ne parat pas avoir vu la ligne qui oceupe le milieu de la bande claire et qui représente, je crois, la membrane de cellule. Nuel (121) admet aussi une membrane autour des sphères de segmentation du Petromyzon Planeri. "La membranc existe, dit-il, au complet dès qu'une cellule s'est divisée en deux.... Elle ne prinètre pas entre les deux cellules qui viennent de se 
former par division, mais nne nowvelle limelle nait ici an moment meme de la division, par un processus analogue à celui quion a si bien observe dalls les plantes." (P. 416, en note.)

OEllacher et plus récemment Ziegler ont lécrit sur le trajet des premiers sillons de segmentation, ehez lit Truite, des vacuoles ou des anfractuosités. Balfour (8) a signalé le même fait che\% les Plagiostomes, et Sirasin (164) chez les Reptiles. Je nai pu observer quexecptionnellement ees anfractuosités el je ne les ai pas trouvées sur des germes qui avaient été traités par laride osmique ou le liquide de Klumunberg. J'ai lieu de croire que ees productions ne sont pis normales et se produisent sous laction de rertains réactifs dureissints. On ne les observe pats lorsquion suit la segmentation sur des ouls transparents, commo erux de l'Epinoche: Van Bencden, Iloffmann. Kingsley et Conn ne les ont pas vues non plus dans les oufs transparents qüils ont examinés.

L'étude des compes du gerne de la Truite, montre qu il se produit de bonme heure, dés le stade VIIl, des sillons paralleles à la surface qui détachent des segments, dans la partie superficielle, pour constituer les premières sphlères do sogmentation. Il se jroduit en mème temps dans la profondeur du germe, atu-dessous de ces premières sphères, des sillons perpendiculaires ì la surlitee ou légèrmment obliques. Ces sillous moins nombreux que les sillons superficiels ne pénedrent pas jusquau vitellus; ils découpent des segments beancoup plus gros que ceus de la surface el qui sont reliés entre cus inferieurement par une masso indivise do protoplasma. Tous ces faits ont été très bien dérrits et figurés pall OAilacher (123).

Lorsque le germe est pirlitgí "n un eertain nombre de segments irreguliers a polygonaus, de tolle sorte quonen compte environ une dizane sur me section nédiane, la segmentation continur à se faire d’une maniè pe plus régulière par une bipartition répétie des cellules: les cellules profondes restant toujours plus volumineuses que les autres.

Les ecllules, qui mesurent à ce moment, sur des pieces fixées par le liquide dr Kloinenberg, "nviron $0^{m+2}$, 0 ' de diamètre, prennent une forme sphéroïlalde at presentent entre elles des lacunes plus on moins grandes of irregulièrment distribues. Cependint les cellules superficielles du germe sont plus rip- 
prochées les unes des autres et constituent déjà une couche spéciale qui se différenciera de plus en plus pendant le développement ultérieur dı germe. His (83) a bien représenté (Pl. 1, fig. 2,3 et 4) eet état lacuniaire dı germe, mais il a figuré aussi (PI. I, fig. 3 ot 4 ot Pl. Il, fig. 4) des chaines de cellules unies entre clles, sous forme de cordons protoplasmiques, moniliformes, plurinucléés. Je n’ai jamais observé une disposition semblable.

La multiplication des cellules marche de plus en plus rapidement, au fur et ì mesure qu'alles deviennent de plus en plus petites, et finalement la masse totale du germe se trouve divisée en un grind nombre de petits éléments qui ont un volume à peu près uniforme. sauf ceux de la couche superficielle, qui restent toujours plus petits et pressés les uns contre les autres, landis quil eviste de nombreuses, lacunos entre les autres éléments.

Noyam.r des sphères de segmentation. - J'ai déjà dit que le premier noyau de segmentation, dont l'origine m'est restée inconnue chez la Truite, est nettement visible dans le germe arant l'apparition dı premier sillon. Ce noỵau a été signalé pour lia première fois par OEllacher (123), en 1872; il ne l'a vu qu une seule fois sur une coupe, et il le décrit comme un eorps gramuleux mesurant $0^{\mathrm{mm}, 08}$ et renfermant un petil corps de $0^{\mathrm{mm}}, 0$. . Ce noyau était bégèrement éxeentrique el plus ratproché de lit surfice que de la base du germe (1). Il a été, au contraire, très hien observé par C. li. Hoffmann 89 dans les cufs transparents du Julis vulgaris, du Scorpana et de quelques autres Poissons marins, oì il se présente entouré d'un aster très not.

Che\% la Truile ce preminr noym nst tres diffeile à observer": il ne peut se roile que sur des conpes el dants less quelgutes cas où j’il pu lo déconvrir, sa présence ne so révélait que par une petite tache in peu plus loncée que le reste du protoplasma et entourée de lignes rayonnantes très étendues. Dans un germe divisé en deur, il est beaucoup plus aisé de voir dans chaque segment un noyau igalement entouré d'un aster. Osillacher

(1) Dans la ligure 17 de la planche X'Xxilt de son mémoire, QEllacher représente ce premier noyau plus pres de la base du germe que de la surface; il y a probablement une erreur dang le texte. 
a vu ces noyaux et lís décrit comme constitués par des amas de corpuseules situes dans une lacune proplasmique multiloculaire. Ces corpuscules mesuraient de $0^{\mathrm{mm}}, 00$ 't it $0^{\mathrm{mm}}, 009$ de dianètre, et se coloraient d'une manière plus intense que le reste du protoplasma par le carmin et le chlorure d'or. OAllacher s'est demandé si eet amas de corpuseules représentait un noyau unique, ou si chaeun de ces corpuseules constituait un noyau. Il a observé une semblable dispositon dans les sphères de segmentation à un stade plus avancé. Charque noyau était formé par un amas de petites vésicules, et au moment de la division de la sphère, l'amas vésiculaire se divisait lui-mème en deux. OEllacher pense que les noyaux ont une membrane plissée formant des lobes, qui sur une coupe optique représentent une série de vésicules placées les unes ì còté des intres. Cet auteur rappelle que Remak (146) a décrit une disposition semblable du noyau dans les sphères de segmentation des batraciens et Lang (110) dans les cellules cancéreuses.

Depuis OEllacher, Hertwig (78) a signalé, comme Remak, dans les sphères de segmentation de la Grenouille, les jeunes. noyaux se formant par la réunion de vacuoles qui résultaient elles-mêmes du gonflement des grains de chromatine (granules de Bütschli); Fol (50), dans les œufs du Toxopneustes lividus, a vu également, à un certain moment, les noyaux constitués par un amas de sphérules, ot il dit s’être assuré que ces corpuscules dérivent directement des renffements intranucléaires ou granules de Bütschli. Trinchese (187) a décrit et représente une disposition semblable dans les globules polaires des Eolidiens; mais, suivant lui les filaments chromatiques du noyau mère deviendraient, jar la réunion de leurs deux bouts, des anneaux qui se réuniraient entre cux et qui, par l'adjonetion d'une nouvelle substance nucléaire, se transformeraient en nouveaux noyaux. Bellonci (17), dans les sphères de segmentation de l'Axolotl a observé aussi la constitution rèsiculaire du noyau ì un certain stade de la cytodiérèse (1). D’après cet au-

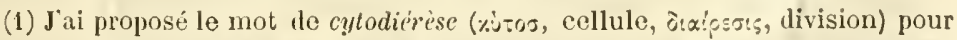
remplacer celui de kariokynèse et jai exposé dans une note spéciale les raisons qui me paraissent devoil faire adopter ce terme. Voir Note sur la division cellulaire ou cytodièrèse, dans les Comples rendus de i'Association françise pour l'avancement des sciences, Congrès de la Rochelle, 1882. 
teur, les éléments cliromatiques s”mbiberaient de suc nucléaire pour devenir des vésicules, qui su fusionneraient entre elles afin de constituer les noyaux-filles. Enfin von Kowalew ski (103) a reconnu que les noyaux des sphères de segmentation du Carassius auratus sont, à l'état de repos, constitués far des amas de petites vésicules arrondies el transparentes.

J’ai vérifié lesobservations d'OEllacher, chez la Truite et celle de Bellonei, ehez l'Avolotl; il est certain que les noyaus se présentent à certains noments comme formés far un amas de vésicules; mais avant d'exposer mes recherches à ce sujet je résumerai ecllesque jail laitessur la division cellulaire daus le gerne de la Truite.

Le processus de la cytodiérèse est très difficile ì suivre dans les premières sphères de segmentation, à canse de l'épaisseur des cellules et de leur état granuleux; de plus, on ne peut arriver à colorer convenablement les noyaux. Si l'on soumet, en effet, un germe de Truite, non segmenté ou seulement segmenté en un pretit nombre de sphères, ì l'action des réactifs colorants, tels que le carmin, le pierocarminate d'ammoniaque, le carmin alumé ou boraté, le vert de méthyle, l'hématoxyline, la safranine, ete., on constate que tout le protoplasma se colore fortement en prenant une teinte uniforme; les noyaux sont à peine un peu plus colorés. Plus les sphères de segmentation deviennent nombreuses et diminuent de volume, moins le protoplasma se colore et plus les noyaux, au contraire, retiennent fortement la matière colorante. J'ai observé le même fait dans les wuls des Batraciens, Grenouille, Triton et Axolotl. 11 me semble résulter de ce fait que la substance elıromatique de Flemming, dont la présence se traduit dans les noyaux par leur affinité élective pour les matières colorantes, est d'abord ì peu près répandue également dans la protoplasma des eellules embryonnaires. Cette substance se sépare graduellement du protoplasma par une sorte de condensation de la substance nucléaire au fur et it mesure qut les noyaur se multiplient.

Cette olfervation que jai consignée dans une communication prélinhinaire à l'Académie des sciences, en 1882 (71), a été confirmée depuis par A. Sabatier (161). "Pour ma part, non seulement, dit eet auteur, je puis confirmer les observations d'Ilenneguy, mais j'ajouterai que dans l’étude que je poursuis 
depuis plusicurs années des éléments reproducteurs, jai été souvent frappé par la difficulté de bien délimitor par la coloration les noyaux dans les ovules màles ou fenelles pendant leur première période de grande activité, c’est-ì-dire alors que ront se produire les éliminations destinées ì déterminer la sexualité de l'élément (p.447) n. Ed. Van Beneden (20) a remarqué aussi dans l'œul" de l'Ascaris megalocephala, que pendant la cytodiérèse la protoplasma devient beaneoup plus chromophile. "Ceci tendrait à établir, dit-il, qu'une partie de la substance chromatique du noyau peut, ì certains moments, se disséminer dans le corps protoplasmique. Si les rapports entre le corps cellulaire et le noyau chromatique sont tels que lia chromatine nucléaire peut se répandre particllement dans le protojhasma, l'on est en droit de supposer aussi que le mème filt peut se produire pour le suc nueléaire. Rien ne s'oppose non plus it l'hypothèse d'après laquelte le phénomène inverse pourrait se produire (p. 584). "

C'est dans un germe du troisième ou du quatrième joul après lia fécondation, qu'on peut suivre le mieux la division des cellules embryonnaires. Celles-ei sont alors constituées par un protoplasma finement grimuleus; leur noyau est assez rohmineux et se colore facilement par les réactifs. Je ne ferai que rappeler brièvement le résultat de mes recherches ì ce sujet.

Le noyau d'une cellule ì l’état de repos contient un réseau formé de petites granulations se colorant fortement sous l'aetion des réactifs colorants; il ne contient pas de nueléole distinct, mais on observe sourent une ou deux granulations plus vohmineuses que les autres. Il est rare dobserver dans un grerme, tué rapidement par les agents fixateurs, des cellules au repos; elles se présentent presque toutes à l'une des phases de la cytodićrèse. Il existe, en effet, autour du noyau un espace clair, duquel partent en divergeant tes lignes très claires qui vont presque jusqu'ì la périphéric de la cellule et dont l'ensemble constitue un aster. Cet aster ne tarde pas ì s'allonger et ì prendre une forme elliptique, le noyau s'allonge anssi dans la même direction. L'aster se divise et ses doux moitiés vont former chacune un nouvel aster aux extrémités du grand axe du noyau. Flemming (47), en se basant sur ins observations sur l'œuf des Echinodermes, n'adnet pas l'existence d'un 
aster unicue entourant d'abord le noyau; pour lui, l'aster est primitivement dicentrique. de ne saurais partager cette manière de voir et les nouvelles observations que jai faites sur ce sujet ne me laissent aucun doute sur la réalité du premier aster unique.

Au moment de la constitution de l'amphiaster, on voit la membrane du noyau se plisser aux deux extrémités de son grand axe, en face des espaces clairs de lampliaster; puis la membrane ne tarde pas à disparaître en ces points et les rayons des asters pénètrent dans lintérieur du noyau. Bobretsky (24) a observé le même fait dans l'œuf de la Nassa, et Fol (50) dans celui du Pterotrachea antour de la vésicule germinative. Flemming, au contraire, nie la pénétration des rayons des asters dans le noyau; suivant lui, les filaments achromatiques du fuseal prennent naissance aux dépens du noyau. Hes propres observations sont identiques à celles de Strasburger, qui fait provenir toute la figure achromatique du protoplasma cellulaire.

Pendant que se passent les phénomènes que je viens do décrire, le réseau chromatique du noyau s'est fragmenté en plusicurs petits corps homogènes, dans lesquels on ne distingue plus les granulations, se colorant plus fortement que celles-ci far les réactil's et ayant la forme de bâtonnets plus ou moins flexucux ou de virgules. Ces petits corps viennent se placer aux extrémités des rilyons des aster's qui ont pénétré dans le noyau et se disposent en une ou plnsicurs rangées, pour constituer la plaque équatoriale des auteurs. La membrane du noyiu disparait complètement et l'on roit alors nettement dąns la cellule la figure bien eonnue du fuseau aree l'amphiaster. Les éléments de la plaque équatoriale se séparent en deux rangées de petits battonnets qui se dirigent chacune en sens contraire, en suivant le grand axe du fuseau, vers chacun des deux asters. Il ma été impossible, même en employant les meilleurs objectil's, tel que le $\frac{1}{18}$ ì immersion homogène de Zeiss, de constater la division longitudinale des batonnets clıromatiques, décrite pour la première fois par Flemming et constatée depuis pir un grind nombere dobservateurs. Cela tient probablement à la petitesse des éléments chromatiques chez la Truite: la scission longitudinale des filaments se voit en 
effet tris bien dans les gros noyaux des larres de Batraciens urodioles.

Arrivés aux extrémités du fuseau, qui a pris alors la forme d'un rectangle et dont les filaments sont parallẻles, les bâtonnets chromatiques, qui ont diminué de nombre, mais dont le rolume s'est angmenté, so groupent de manière ì constituer une figure pectiniforme. Lors de mes premières recherches sur le eytodiérìse, je eroyais que les éléments chromiltiques se fusiomnient entre eux, en commencant par leurs extrémités périphériques. Flemming a réfuté avec raison cette interprélation; jai reconnu depuis que les éléments chromatiques restaient libres, tout en se rapprochant cependant parr leurs parties périphériques, et s’ils paraissent souvent fusionnés, c'est qu'ils ont été agglutinés par le réactif fixalcur, ou bien parce qu'on ne les examine pas avec un grossissement suffisant.

Le eorps de la cellule ne commence à s'étrangler en son milieu, qu’après la division du noyau; les rayons des asters out alors en partie disparu; mais les filaments connectifs qui réunissent les deux moiliés du noyau persistent jusqu'à la séparation complète des deux nouvelles cellules.

D'après Flemming, le nouveau noyau de la cellule fille parcourrait en sens inverse, pour arriver au repos, les mêmes phases que le noyan-mère. Il est difficile de suivre, dans les cellules du germe de la Truite, les transformations du noyaufille; mais les faits que jai observés dans les cellules de segmentation del'Axolotl et du Triton et qui corrohorent tout it fait ceux que Bellonci a décrit dans ces mêmes cellules, tendent à me fiure croire qưil en est de même chez la Truite et que le noyau-fille se forme d'une façon différente de celle adınise par Flemming. Dans les sphères de segmentation de l'Axolotl et du Triton, les filaments clromatiques, qui doirent constituer le noyan-fille, sont disposés en anses ì peu près parallèles dont les extrémités libres sont dirigées du côté du plan de séparation des deux cellules. Les anses sont formées par deux filaments parallèles très déliés. Bientôt les extrémités libres de chaque anse se rapprochent de manière ì former un anneau; le filament interne disparait et ì sa place on voit des granulations accolées à la face interne de l'anneau. Les anneaux augmentent de volume, se rapprochent les uns des autres et s'aceollent sur 
certains points. Comment les anses chromatiques se transforment-elles en vésieules, ainsi que l'a vu Bellonci, e'est ee que je ne puis dire actuellement; mais il est eertain qu'ì un stade un pen plus avaneé, le noyau-fille est formé par l'agglomération des vésieules qui se fusionnent petit à petit et constituent finalement le noyan définitif avec son réseau intérieur.

Jo ne veux pas insister iei sur cet intéressant phénomène, je ne l'ai décrit sommairement que pour pouvoir expliquer la disposition signalée par OEllacher dans les premières splıères de segmentation de la Truite, disposition dont j'ai vérifié l'exislence, ainsi que je l'ai dit plus haut. L'amas formé de plnsieurs petiles vésicules, qui constitue le noyau de ees sphères, n'est qu'un stade de transformation du noyau-fille. Il est probable que le mème processus doit avoir lieu dans les cellules plus petites, mais le faible volume des éléments chromatiques n'en permet pas la constatation.

Parablaste. - Les phénomènes qui se passent dans la conche sous-jacente au germe, au moment de la segmentation, sont très intéressants à suivre et présentent une grande importanee au point de vue de l'embryologie générale. Cette couche, qui a regu des noms très différents suivant les anteurs, ne parait ètre qu'un épaississement de la couche corticale, qui enveloppe le vitellus, et que jai déjì décrite dans l’ouf non fécondé. Je rappellerai brièvement les opinions émises sur cette eouche.

Lereboullet (111 et 112), qui la signala le premier, lui donna le nom de feuillet muqueux; il constata son existence chez le Brochet, la Perche et la Truite, et déerivit les transformations qu'elle subit pendant l'extension du germe sur le vitellus. Cette couche renfermerait, d'après lui, des cellules provenant de la transformation des globules vitellins.

Kupffer (104) a vu chez l'Épinoche des séries régulières de noyaux au-dessous du germe et des cellules prendre naissance par formation lil re autour de ces noyaux. Aussi a-t-il domné le nom de zone nueléaire (Kernzone) à la couclıe située au-dessous du germe.

Owsjannikow (124) a remarqué aussi sous le germe du Coregonus lavaretus une couche protoplasmique dans liqquelle apparaissont des cellules qui prennent part it la formation de l'embryon. 
Van Bambelie (11) a constité, dans l'œuf du Gardon(Leuciscus mulilus), que le germe segmenté est séparé du vitelus pir une conche partieulière, phus épaisse à la périphérie qu'au centre, cette couche intermédiaire est formée par un protoplasma à granulations nombreuses, plus grosses que celles des cellules de segmentation. Le bourrelet périphérique renlerme des noyaus ovalaires à grosses granulations, qui se colorent plus facilement que les noyaux des cellules. Il existe aussi des noyaux dans la partie centrale anineie. Van Bambele pense que cette couche provient du germe et que les noyaux qui s'y trouvent dérivent peut-être du premier noyau de seguentation; "les cellules, dont ils constituent les centres, résultent aussi du proerssus de segmentation, se faisint ici avec plus de lenteur que dans le germe proprement dit. " Suivant lui, la couche intermédiaire se constitue alux dépens de la couche protoplasmique qui entoure le vitellus de l'œul arrivé à maturite, et il ajoute que "lorsque la couche internédiaire existe, le mantean protoplasmique a positivement disparu autour du globe vitellin."

Weil (195) et OEllacher n'admettent pas de couche intermédiaire dans l'œuf de la Truite. Cependant OEllacher a bien vu et figuré une couche renfermant des cellules au-dessous du germe, mais senlement à un stade dejjà avaucé, lorsque le blastoderme s'est étalé et soulevé an-dessus du vitellus; suivant lui, il se détacherait alors du toit de liı cavité sons-germinative des cellules qui pénètreraient dans le vitellus et s'y multiplieraient, mais ne joueraient aucun rôle. OEllacher regarde le germe comme étant en continuité avec la membrane vitelline (couche corticale); celle-ci se composerait de deux couches, l'une superficielle, dépourvue de graisse. l'antre profonde, charges de gouttelettes huileuse's; le germe serait compris entre ces deux couches, il a ru quelquefois au-dessous du germe une couche mince grossièrement granuleuse, ec ressemblant au protoplasma germinatif.

lilein (94) a bien étudié la constitution de la couche intermédiaire chez la Truite et lui a donné le nom de parablaste par opposition ì l'archiblaste qui, pour lui, constitue le germe; mais il a reconnu que le parablaste et le germe sont en continuité. 11 a décrit avec soin les noyaux qui se forment dans cette couche 
et il admet que des cellules prennent naissance dans le parablaste pour venir s'ajouter an germe segmenté. Klein assimile l'œuf des Poissons osseux à une cellule graisseuse : le parablaste représente le manteau protoplasmique de la cellule; le vitellus est le globule graissenx.

His (82) donne au parablaste le nom de rempart germinatif (Keimwall); les noyaux qu ïl renferme préexisteraient à la fécondation et viendraient des leucocytes qui ont pénétré dans l'œuf ovarien.

Hiecliel 63 ) semble avoîr méconnu complètement le parablaste dans les œufs transparents qu'il a examinés. Gœtte (58) et Calberla (31) ont, au contraire, constaté l'existence d'une zone nucléée au-dessous dı germe de l'oissons osseux.

Ed. Tan Beneden (19) a décrit une couche intermédiaire dans l'œuf d'un Poisson marin indéterminé de la famille des Gadides. Dans cette couche apparaissent par roie endogène des noyaux entourés de stries rayonnantes et qui deviennent les centres de formation de cellules, qui s'ajoutent ì celles du germe segmenté.

Hoffmann (89) a retrouvé le parablaste dans tous les cufs de Poissons marins qu'il a examinés, et il a pu dans ces ouls découvrir l'origine du premier noyan du parablaste. Après la formation du premier noyau de segmentation, résultant, conme on sait, de la fusion du noyau spermatique avee le pronucleus femelle, ce noyau se divise en deux, perpendiculairement ì la surface du germe: l'une des moitiés reste dans le germe et derient lorigine des noyaux des sphères de segmentation, l'autre moitié émigre dans le parablaste et donne naissance aux nombreux noyaux fu'on observe dans cette couche. Suivant Hoffmann, ì chaque bipartition successive du noyau du germe, correspond une semblable bipartition dı noyau du parablaste; do telle sorte, que, au moins au début du développement, il y iurait autant de noyaux dans le parablaste qu il y en a dans le germe. Les noyaur parablastiques se multiplient comme ceux dı germe par division indirecte, en présentant les phases que j’ai exposées plus haut.

Ziegler (200) a constaté anssi récemment l'existence du pa rablaste dans l'ouf des Salmonides et dans ceux du Rhodeus amarus et du Syngnathe. Enfin, Kingsley et Conn (93), Ryder 
(154), Agissiz ot Whitmin (1), elc.. l'ont décrit dills plusieurs espèces marines. Ryder tomne à colte couche le nom d'hypoblaste vitellin (Telli-Hypoblast). Agassiz et Whitman celui de périblaste. Le terme de parablaste ayant été adopté par la plupart des auteurs, e'est le nom que je conserverai à cette couche importante.

L'ouf des Salmonides est filvorable pour l'étude du dévelog pement du parablaste, eefle conehe ne devenant bien apparente qu'a un stade avancé de la segmentation. Dans un onf non segmenté ef pendint les premiers stades du fractionnement. le gernie repose directenent sur le vitellus, dont il est séparé. ainsi que je laai dejà dit, par une ligne très nette. Sur des ouf: durcis par l'acide chromique. on peut facilement enlerer lo germe au moyen flume aiguille, en exerçant une légère pression au-déssous do lui : il se détache alors avec une petitr zone circulaire de la couche corticale. Il en est de même dims les wufs traités par l'acide osmique on le liquide piero-acétique; cn ourrant l'œuf dans l'eau aciduléc, la masse vitelline restée fluide se dissout et le germe se détache entouré d'une zone présentant une consistance plus grande que le reste de la couche corticale. (Planches, fig. 61.)

Des coupes praliquées sur des germes ainsi détachés et colorés montrent que les bords du disque germinatil se continuent arec une zone protoplasmique, finement granuleuse, ayant le même aspect que le germe et présentant sous l'influene des réactifs la mème coloration que lui. Celte zone est it la surfare du vitellus et n’existe qu’ì lia périphérie du germe où clle présente son maximum d'épilisseur : clle va en s"umincissant jour devenir à peu pres invisible à une distance qui corresfonel an quart environ du diametre du germe. Cette zone, qui est formée en somme par umr expansion du protoplasma germinatif, est l'origine du pirablaste; je la désignerai sous le nom dr zone périphérique; alle se continue avec la couche corticale enveloppant lo vitellits. Elle ne diffère de cette dernière que fur son épaisseur plus gramle, et la consistance qu’elle jrend sous linfluence des ríatils coagulants.

Pendant les premiers states de la segmentation, lorsque les cellules profondes sont encore voluminenses, la zone póriphérique ne subit aucune modification; elle reste en continuité 
avec le germe. Dès que les cellules profondes commencent à se délimitre nettentent les unes des autres, la zone périphérique se separe aussi du germe. C'est à ce moment quaplparaissent dans cette zone des noyaux semblables ì ceux des cellules de segmentation. Ces noyaux, d'abord rares ol très rapprochés de liı périphérie du germe, sont souvent entourés d'un aster. Bientot on les voit se multiplier par division indirecte en présentant toutes les phases qui s'observent dans les cellules provenant de la segmentation du germe : presque tous ces noyaux présentent simultanément les mèmes platses de la division. Clest rers la fin du troisieme jour et le commencement du quatrième, que le phénomène est le plus visible. A ce moment, la zone périphérique pénètre au-dessous du germe et sitend, comme la très bien vu Van Bambelie (11), de la jériphérie vers le centre; elle finit par former an-dessous du germe une couche continue qui constitue le parablaste.

Le parablaste, une fois différencié, est donc une couche protoplasnique plurinuclée, ayant la forme dime sorte de criltère i bords très nets dans lequel est enchassé le germe segnenté. En lehors de eclui-ci, il continue à sétendre sur le vitellus jusqu à une certaine distanec, comme le faisait la zone périphérique. Du còté du vitellus, le parablaste est mal délimité: il s'enfonce plus ou moins entre les globules lunileux acemmulés sous le germe; il se mélange am grosses granulattions et aux glohules albumineux de la couche rorticale.

On peut bien apprécier la forme du parablaste en chassant aree le pincean les cellules du germe sur un wenf durci; il reste alor's une cupule arrondic qu'on peut examiner par transparence, après lauvoir montée dans le baume de Camadia. (Planches, fig. 6't.)

Le parablastr eproure vis-i-ris des réactifs colorants les mèmes modifications que le protoplasma du germe. Au fur et à mesure que les noyau se multiplient, le protoplasma palratblastique se colore de moins en moins; la substanee chromalique, d'abord liffuse, se concentre de plus en plus dans les noyatus.

Las premiers noyaux du parablaste ont le mème aspect que ceur des cellules de segmentation, et ils conservent eet ispect tant que dure leur nultiplication par roie indirecte; mais ce 
mode de division ne dure que peu de temps; à partir de la fin du quatrième jour, il n’a été impossible de trowver dams le parabliste un seul noyau présentant des figures cytodiérétiques. Du reste, à partir de ce moment les noyatux ont changé d’aspeet; ainsi que l'a bien vu klein (94), ils sont beatcoup plus volumineux que ceux du germe, leur contour est irrégulier, et ils renferment un réseau chromatique très net et à larges mailles. Balfour (8), chez les Plagiostomes, n’a vu aussi des figures cytodièrétiques dans le parabliste qu'iu début de la segmentation.

Le parablaste, comme nous le verrons bientòt, suit l'extension du germe ì la surface du vitellus et finit par oecuper une large surface; il renferme toujours de nombrems noyaus, qui se multiplient alors par division directe, et subissent plus tard, dans certaines régions, dlintéressintes modificalions que jo décrirai à propos du développement de l’embryou.

Lorigine des noyaux du parablaste aus dépens du premier noyau de segmentation, établie par Hoffmamn pour les culls de ecrtains Poissons marins, est tout à fait inadnissible pour la Truite, puisquil n'existe pas trace de parablaste au-dessous du germe, pendant les premers stades de la segmentation. On serait done tenté d'admettre, conme l'ont fait les premiers observateurs, entre autres Kupfer(104), Klein(94), Van Beneden (19), que les premicrs noyaux du parablaste apparaissent par formation libre: mais on sait, d’après tous les travaux récents sur l'origine et la structure des éléments cellulaires, que la formation libre de noyaux ou de cellules est un fait très rare, si toutefois il existe, et les histologistes molernes ont établi d'une façon à peu près certaine que tout noyau provient d'un noyau préexistant. Il me semble done plus logique d'admettre que les premiers noyaux parablastiques de la Truite viennent des noyaux du germe. Il est probable que, lorsque la zone périphérique se sépare du germe, des noyaux provenant des cellules de segmentation limitrophes pénètrent dans son intérieur; il il se passe là, à un stade utérieur, le mème pluénomène que Hoffmann a observé avant le début de la segmentation. Mais tandis que, chez certains Poissons, le parablaste se sépare de très bonne heure du germe et sur toute son itendue, chez les Salmonides il ne so différencie que plus tarl et seulement d'it- 
bord à lit périphérie. Ce fait ne samrait nous surprendre, puisquion observe souvent un plénomène semblable en embryogénie. On sait, en effet, qu'un même organe peut apparaitre plus ou moins tardirement clez différents animaux, sans que jour cela l'évolution de l'embryon soit modifiée.

Le parablaste doit donc être considéré comme une portion du germe qui ne prend pas part ì la segmentation, et dans laquelle di multiplication des noyaux n'est pas suivic immédiatement de la division de la masso protoplasmique. Je dis immédiatement, parec qu en effet on voit apparaître, à un moment donné de véritables cellules dans le parablaste.

Liuffer (104) le premier constata que dans l'œuf de l'Épinoche il se forme des cellules autour des noyaux du parablaste, et que ces cellules viennent s'ajouter à celles du germe. Klein (94) a ru aussi des collules naître dans la conche parablastique, et, d'après lui, elles formeraient l'endoderme. Van Bambeke (11) pense également que l'endoderme provient de cellules formées dans la conclie internédiaire. Ed. Yan Beneden (19) admet une formation endogène de cellules dans la couche parablastique; la striation rayonnée du protoplasma autourdes noyaux prouve, d'après lui, la formation de cellules. Du reste, il a vu des cellules engagées dans le parablaste faire saillie dans la cavité germinative. Balfour (8), chez les Plagiostomes, a vu aussi, andessous du germe segmentí, dans le vitellus, des noyaux en roie de division; ces noyaux deviennent des centres de formation. de cellules, qui pénètrent dins le germe et entrent plus tard dans la constitution de l'endoderme. OEllacher (123), ainsi que je l’ai déjà dit, tout en reconnaissant l'existence des cellules dans le vitellus sous-jacent au germe, pense que ces cellules proviennent du germe et ne jouent aucun rôle. Hoffinann '89), à l'encontre des auteurs cilós précédemment, admet, aussi bien pour les P̉agiostomes.que pour les Téléostéens, que toutes lea cellules du germe dérivent de larchiblaste et que le parablaste ne participe en rien a leur formation.

Les recherches multipliées que j’ai faites sur l'évolution du parablate, mont conduit anx mêmes conclusions qur hiupffer. Ë. Van Benedru et Balfour. C'est sur les œufs d'Epinoche vivants, damnines pal transparence, quon peut le micux suire li genise des cellules parablastiques. Environ vingt-cinq leures 
après la fécondation, lorsque la segmentation est dijjì issez. avancée, on voit nettement au-dessous du germe, la zone nucleaire de liupffer. Dans cette zone les noyaux les plus rapprochés du germe sont entourés de lignes claires, radiées, séparées par des lignes formées de grosses granulations protoplasmiques. Autour de quelques-uns de ces systèmes, on voit une ligne do contour très nette, qui correspond à une membrane cellulaire: certains d'entre enx ne présentent la ligne de contonr que du còté du germe et sont encore engagés du còté opposí dans la masse protoplasmique, conme l'a très bien dícrit Ed. Van Beneden. Si l'on traite l'œuf par l'acide acétique dilué, on voit appariitre immédiatement dans la zone périphérique du parablaste des figures cytodiérétiques plongées dans le protojlisma; plus près du germe. Ies noyaux sont ì l'état de repos, mais ils sont entourés d'un aster: c'est autour-de ces noyauxque sorganise une membrane cellulaire. Il résulte de cette disposition qu'il n'y a pas de limite nette entre le germe ot le pilrablaste; cette linite est constituce par les cellules en voie de formation qui se détachent du parablaste pour s'ajouter aur germe.

La genèse des cellules parablistiques ne jarait pas durep longtemps; dess que le germe commence ì s'étaler à la surface du vitellus, que la cavité germinative s'est constituée et que les fouillets blastodermiques se différncient, on 11 roit jlus de cellules prendre naissimee en dehors du germe.

Che\% la Truite, il est impossible de suivre directenent les phinomines que je viens de décrire, et cost seulement sur des coupes quion peut voir, comme le montre la figure 6.3 Panches, des mamelons, renfermant chacun un novan entouré d'un aster, faire saillie an nivean du bomrelet parablastique, et proéminer parmi les eollules marginales du disque germinatif. De mème que cluz l'Euinoche, la formation les exllules parablastiques a une durér très linitée et ne sobserve guère rue prodant le statle peprésenté figure 4 (1Planches). Les cellules d'origine parablastique ont $m$ anject identique ì celui des cellules de segmontalion; dès qu'elles sont mểées ì celles-ci, on ne peut plus les distimguer. Il me semble done impossible

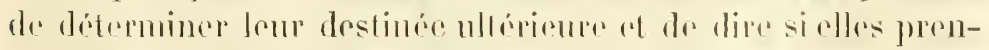

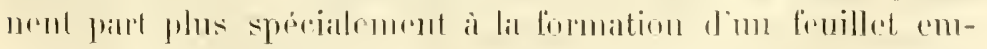


bryonnaire. Pour ma part, je suis lout disposé ì croire que ces cellules ne jouent aucun rôle spécial dans le développement embryonnaire. Elles sont dabord peu nombreuses relativement aux cellules archiblistiques, de plus clles sont constituées par la même substance que les éléments du germe, puisque le parablaste n'est qu'une portion du disque germinatif: enfin leur mode de genèse, différent en apparenee de celui des cellules embryonnaires, n'est qu'une continuation du processus de segmentation. Les premiers segments se détachent, en effet, du disque germinatif de la mêne manière que les cellules parablastiques se séparent du parablaste; dans le premier cas, les cellules sont très volumincuses par rapport à la masse parablastique, dans le second cas, elles sont beaucoup plus petites.

Von liowalewiski (103) est arrivé à formuler une manière de voir analogue d'après ses recherches sur différents Poissons. Il ¿ vu que la segmentation peut commeneer de bonne lieure, lorsque le disque germinatif ne comprend encore qu'une partie du protoplasma de l'œuf; la conecntration du protoplasma se continuant longtemps dans le parablaste, celui-ci donne naissance à un grand nombre de cellules qui s'ajoutent au germe. Si la concentration du protoplasma est au contraire terminée an début de la segmentation, très peu de cellules se formeront dans le parablaste.

Depuis la note préliminaire dans laquelle javançais ces faits (72), Ziegler (200) a observé aussi des saillies du parablaste parmi les cellules profondes du germe; nais il n'a pas constaté l'existence de noyaux dans ces mamelons, et il doute qu'il y ait là formation de cellules. Kingsley et Conn (93) ont figuré des cellules en voje de formation dans le parablaste autour des noyaux dans un neuf de Merlucius. G. Brook (27) a observé le même fait chez le Trachinus vipera. Plus récenment Agassiz et Whitman (1) ont vu chez le Ctenolabrus, les noyaux du parablaste provenir, ánsi que je lai indiqué pour la Truite, des collules de la périphérie du germe. Von Kowalewsky (103) a fait la même ohservation sur le Carassius auralus. Wencliebach (197) fait dériver aussi du germe les noyaux du para= blaste, mais ils peuvent aroir pour origine, anssi bien les noyaux des cellules périphériques du germe que ceux des cel- 
lules qui tombent du toit de la cavité germinative. Enfin List (116) a constaté également elıez le Crenilabus pavo que ces noyaux avaient pour origine les noyaux des cellules marginales du germe.

Il est intéressant de rapprocher les phénomènes qui s'observent dans le parablaste de ceux qui se passent dans le sac embryonnaire des végétaux. On sait que chez les Angiospermes, après la formation de l'oosphère, des vésicules synergiques et des vésicules antipodes, et lorsqur l'oosphère est fécondée, le noyau définitif du sac embryonnaire se multiplic par division indirecte et donne ainsi naissance à un grand nombre de noyaux contenus dans la eouche protoplasmique, qui revêt la fice interne du sac. Plus tard seulement ees noyaux deviennent des centres de formalion des cellules; il apparait entre eux des filaments connectifs sur le milieu desquels se forment des plaques cellulaires et des cloisons cellulosiques, qui délimitent les cloisons du futur alhumen. Dins le revêtement pariétal du sac embryonnaire de cerlaines Légumineuses, d'Orobus et de Pisum, et dans le suspenseur des embryons de ces mêmes plantes, Guignard (62) et Strasburger (185) ont vu que ces noyaux, qui s'étaient multipliés par division indirecte, subissent une fragmentation ou une division directe, sins devenir des centres de formation de cellules. La même succession de faits s'observe dans le parablaste. Les noyaux s'y multiplient d'abord par division indirecte, puis autour de certains d'entre eux se différencient des cellules, enfin plus tard les autres noyaux subissent seutement des divisions directes ou des fragmentations, comme nous le verrons, à un certain stade du développement de l'embryon. Ce fait est important au point de rue de la biologie cellulaire; il prouve, en effet, que chez les animaus comme chez les régétaux, la division du noyau et celle de la cellule sont deur phénomènes qui, bien que généralement intimement liés l'un à l'aulre, sont néanmoins indépendants.

Lorsque le parabliste est constitué au-dessus du germe, et antour de lui, il continue à s’étendre en même temps que le blastoderme et finit par recouvrir entièrement le vitellus; jöindiquerai plus loin les transformations quil subil et le rôle qu'il joue pendant le développement de l'embryon.

Cavilé germinulixe. - J'al laissé le germe au moment où 
il est constitué par un amas de cellules nombreuses, semblables entre elles, excepté à la surface où elles forment une couche déjà nettement différenciée. La forme que présente alors le disque germinatif, lorsquion l'observe sur une section médiane, présente des variations. Le plus souvent le germe fait saillie au-dessus du vitellus sur lequel il repose par une base i peu près plane; d'autres fois, au contraire, il est situé dims une sorte de cuvette creusée dans le vitellus, de telle sorte que sa face profonde est convexe et sa surface libre presque pline. C'est sous cet aspect que certains auteurs, entre iutres Ziegler (200), ont représenté en coupe ce stade de la segmentation chez les Salmonides. Cette disposition n'est pas normale, on ne l'observe jamais sur les œufs transparents dont on peut suivre le développement à l'état vivant; elle est due à une compression exercée sur le vitellus par la capsule lors du durcissement, compression qui a pour effet de déformer le germe et de le faire pénétrer dans le vitellus.

Vers la fin du troisième jour après la fécondation, le germe commence à s’étendre sur la masse vitelline. Jusque-là son plus grand diamètre, mesuré sur des œufs durcis par liacide chromique, était depuis le commencement de la segmentation d'environ $1^{\mathrm{mm}}, 2$ à $1^{\mathrm{mm}}, \ddot{\jmath}$. Au quatrième jour, le germe mesure $1^{\mathrm{mm}}, 9$; au cinquième, $2^{\mathrm{mm}}$, au sixième, $2^{\mathrm{mm}}, 7$, au septième, $3^{\mathrm{mm}}$. En même temps que sa surface augmente, son épaisseur diminue. Avant daborder la description des transformations que subit alors le germe, je dois dire quelques mots d'une question sur laquelle les embryogénistes sont loin d'ètre d'accorl.

Lorsque le parablaste śest étendu au-dessous du germe de manière à former sous lui une couche continue, on voit la face profonde du disque germinatif se soulever au-dessus du vitellus; le soulèrenunt rommence à une petite distance des bords, ceux-ei et le centre du germe continuant à reposer sur le parablaste; il en résulte un espace circulaire vide de cellules qui occupe toute la périphéric du germe. Bientôt le centre du germe s'amineit, probablement par migration de cellules vers le bord, et se soulève alu-dessus du vitellus; il existe alors entre le vitellus et le germe un espace ville qui est le eommencement de la carité germinalive. 
Celte cavité a été vace chez les Poissons par lat plupart des auteurs, Stricker (186), Rieneck (147), Weil (195), OHllacher (123), Klein (94), Owsjannikow (124), Van Bambelie (11), Van Bineden (195), His (83), Hoffmimn (89), Ziegler (200), ete., mais quelques-uns d'entre cux ont admis en outre diuns l'épaisseur du germe une autre cavilé qui correspondrait à la cavité de segmentation des Amphibiens, des Ganoïdes, des Plagiostomes et des Cyclostomes. C'est ainsi que Lereboullet (111-112), décrit le germe du Brochet, de la Perche, at de la Truite comme une vésicule creuse, et il pense que le fait est général pour les P'oissons. Kupffer (104), a ru quełquefois une carité dans le grrme coagulé du Gobius minutus, mais il s'est demandé si ce n’était pas là le résultat des réactifs. Yan bambeke (11) a observé, chez le Gardon, vers la fin de la segmentation, dans l'épaisseur du germe, une cavité qui, sur des coupes, présentait la forme d'un croissint à courbures sensiblement parallèles à colles du germe, et à jarois irrégulières. Il a trouvé constamment cette cavité de segmentation et la considère comme normale.

Depuis Lereboullet, Je seul observateur qui ait décrit une caviti de segmentation, cluez les Salnonides, est Ziegler (200).

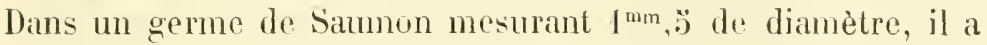
trouvé une cavité de $0^{\mathrm{mm}}, \tilde{s}$ de diamètre et de $0^{\mathrm{mm}}, 2$ de hauteur; elle était située excentriquenent et son toit itait formé par un petil nombre de conches de cellules, tamdis que son plancher était beaucoup plus épais. Ziegler dérit aussi une cavité de segmentation dans lo germe de la Truite, du Rhodeus amarus et du Syngnathe. Plus tard cette cavité s'élargit vers le vitellus et les cellules qui formaient son plancher s'écartent, de sorte que la cavité de segmentation repose alors sur le vitellus et devient cavité germinative.

J'ai cherehé l'existence d'une cavité de segmentation sur un grand nombre de coupes de germes de Truite it différents stides. Jo n’ai vu qu'une seule fois, sur un couf durci par l'acide clromique, une cavité répondant à peu près, par sa forne et prar sa situation, à celle qui a été décrite par Van Bambelie et par Ziegler. Sur tous les autres germes que j’ai examinés, il n'y avait pas trace de cavité interne. Mais à un stade peut avancé de la segmentation, tel que celui représenté figure 63 (Planches), j’ai 
trouvé quelquelois un sspace irrégulier, vide de cellules, qu'on pourrait considérer comme une cavité de segmentation. Son existence étant loin d'être constante, je crois pouvoir dire avec la majorité des auteurs. qu'il n'y a pas de cavité de segmentation chez la Truite. Il est possible qu'il en existe une chez d'autres poissons, chez le Gardon, le Rhodeus amarus, le Syngnathe etc., mais l'absence ou la présence de cette cavité ne me paraissent pas avoir une grande importance, ainsi que je le démontrerai lorsque je comparerai le développement des Téléostéens à celui des autres Vertébrés.

Si l'existence d'une cavité de segmentation est encore douteuse chez les Poissons osseux, celle d'une cavité germinative, e'est-à-dire d'une cavité située au-dessous du germe, est admise par tous les embryogénistes. Certains auteurs cependant ont confondu les deux cavités; ainsi Stricker, Klein et Hoffmann appellent cavité de segmentation, la cavité placée sous le germe.

La formation complète de la cavité germinative coïncide avec le commencement du développement de l'embryon. On peut, en effet, faire remonter à ce stadc l'apparition des premiers feuillets embryonnaires. Quand le germe segmenté s'est étalé ì la surface du vitellus, et que la cavité germinative oceupe toute la portion centrale, le toit de la cavité est formé par cinq à six couches de celhules situées au-dessous de la couche superficielle, qu'on peut appeler couche enveloppante ou, avec OEllacher, couche cornće. Ainsi que je l'ai déjà dit, les bords du disque germinatif reposent sur le vitellus, ou du moins sur le parablaste; ces bords constituent le bourrelet germinatif qui se distingue très bien sur des germes vus en surface et montés dans le baume du Canada, où il paraît plus opaque que le germe. Ce bourrelet est formé par une masse cellulaire qui, de très bonne heure, est plus épaisse et plus large d'un côté du germe que de l'autre. Il en résulte que le centre de la cavité germinative ne correspond pas à celui du germe.

La partic épaissie du bourrelet germinatil esi celle sur laquelle va se développer l'embryon. Elle est déjà très nette sur des germes mesurant $2^{\text {mu }}, 8$ de diamètre à la fin du sixième jour. Une coupe diamétrale du germe, faite perpendiculairement ì la partie épaissie du bourrelet, montre qu'en ce dernier point les cellules profondes du germe sarancent dans la cavité germi- 
nalive d'une manière irrégulière (Planches, fig. 89). Un asse\% grand nombre de ces cellules se séparent du bourrelet et sont disséminées sur le plancher de la cavité. Pour employer une comparaison qui rend bien l'aspect du germe ì ce stade, vu sur une coupe, on peut dire que lo disque germinatif représente une voûte très surbaissée, dont les piliers se seraient en partie écroulés et dont les pierres formeraient un talus ì la base des piliers. Des pierres de ce talus auraient roulé sur le plancher de la voûte jusqu'à une certaine distance. Cette image correspond du reste à peu près à ce qui se passe dans le bourrelet à ee moment; mais comme ce processus correspond à la formation des feuillets embryonnaires, j’exposerai brièvement, avant de donner les résultats de mes reclierches à ee sujet, les principales opinions qui ont été émises par les auteurs sur cette question, la plus controversée de l'embyrogénie des Poissons osseux.

\section{VI. - Formation des feullets buastonermiques.}

A l'exemple de Gotte (58) et de Van Bambeke (11), qui a fait un très bon listorique de la question, on peut diviser en plusieurs groupes les opinions des embryologistes relativement à l'origine des feuillets enibryonnaires des Poissons.

On peut d'abord établir deux grands groupes: dans le premier se rangent tous les auteurs qui font provenir du germe segmenté les trois feuillets du blastoderme; dans le sceond groupe, eenx 'qui n'en font provenir que deux, le troisième feuillet dérivant du parablaste. Chacun de ces groupes admet des subdivisious qui comportent les différentes opinions émises sur le mode de formation de chaque feuillet.

I. - Les premiers anteurs qui ont abordé l'embryologie des Poissons osseur, Rathlie (135) et von Baer (4), n'ont étudié les oufs qu'à l'aide de méthodes tout à fait imparfaites, et malgré cela ils sont arrivés à des résultats qui eoncordent à peu près entièrement arec ceux des auteurs les plus récents. Les deux célèbres embryologistes admettaient que le blastoderme se partage d'abord en deux fenillets, l'un superficiel, ou feuillet séreux (ectoderme), l'autre profond, feuillet nuqueux (endoderme); de ce dernier se sépare plus tard le feuillet moyen ou vasculaire (mésoderme). 
C'est à cette opinion que se sont rangés plusieurs observateurs. Stricker, Rieneck, OEllacher, His et IIoffmann font, en effet, dériver les trois feuillets du germe. Après la différenciation de la couche superficielle, membrane enveloppante, la masse du germe se sépare en trois couches qui sont les trois feuillets; ces autenrs diffèrent cependant entre eux sur des points secondaires. Ainsi Stricker, Rieneck ef Weil admettent que des cellules tombent de la voûte de la cavité germinaiive sur le plancher et émigrent ensuite vers le bourrelet périphérique. Obllacher pense au contraire que ces cellules pénètrent dans le vitellus, s'y multiplient et forment les noyaux du parablaste; jai déjà démontré l'erreur d'OEllacher à ce sujet.

b'autres embryologistes, qui font provenir également les feuillets blastodermiques du germe segmenté, assignent à la formation de ces feuillets un mode différent de la simple différenciation admise par les auteurs cités précédemment. Gœite (60), le premier, observa qu'après la formation de la couche superfieielle (Deckschicht), la couche fondamentale du gerne (Grundschicht) ou couche blastodermique primitive (primitive heimschicht), se recourbe sur ses bords dans l'intériem de la cavité germinative et donne naissance à une couche blastodermique secondaire (secundure Keimschicht). La partie non réfléchie de la couche blistodermique primitive constitue l'ectoderme; la partie infléchie se sépare plus tard en deux autres couches, dont l'une est le mésoderme et l'autre l'endoderme.

Telle est aussi l'opinion d'Hreckel (63). Pour lui, l'endoderme résulte d'une invagination des bords du disque germinatif; quant au mésoderme, il aurait une double origine; il viendrait en partic par délimination de l'ecloderme et en partie par la migration de cellules amiboïdes de l'endoderme.

Dans une note jrésentée à la Sociélé philomatique, en 1880 , je faisais connaître le résultat de mes recherches sur le développement des feuillets blastodermiques chez les Poissons osseux, et je me rangeais complètement à l'opinion de Gœtte (69).

Depuis lors, deux auteurs américains, Kingsley et Conn (93), ont vu aussi la réllexion du disque germinatif pour donner naissance à l'endoderme, dans des œufs de Poissons marins (Ctenolabrus, Merlucius); mais, contrairement à ce que j'avais avancé, ils admettent que la couche superficielle (membrane 
enveloppante) prend seule part à la rétlexion. Zliegler (200) a recommu récenmont la réffevion des bords du germe ehez le Saumon, la T'ruite et le Rhodeus amarus. Goronowitseh (57) idduet également la réflexion de l'ectoderme, sans partieipation de la conche envelopyinte.

II. - Les embryologistes du second groupe, ceux qui font dériver le feuillet interne du parablaste, sont moins nombreux que ceux qui font provenir tous les feuillets du germe segmenté : ce sont Vogt (191), Lereboullet (111), Kupffer (104), Owsjannikow (124), Klein (94), Van Bambeke (11), Ed. Van Beneden (19), Agassiz et Whitman (1), et Brook (27).

Vogt qui, dans son travail sur l'embryologie des Salmones, n'attachait pas une grande importance à la distinction des dillérents feuillets, est peu explicite sur l'origine de ces feuillets: parlant des eellules qui forment llintestin, il se demante si elles sont baignées par le liquide vitellaire on si elles en sont séparées yar une membrane. Lereboullet est beaucoup plus alfirmatil et admet que le feuillet muqueux n’a dans l'origine aucune connexion avec le blastoderme. liupffer rapproche la zone nucléaire (parablaste), quil a observée chez l'Epinoche, du feuillet muqueux de Lereboullet, mais il ne se prononce cependant pas catégoriquement sur l’origine véritable, ni sur l'époque d'apparition de l'endoderme. Ow sjannikow admet que les cellules sortent du parablaste el viennent se disposer entre le germe et le vitellus en une rangée régulière qui seusble constituer un feuillet spécial, sur la nature duquel il ne se prononce pas. Van Bambeke est plus précis dans ses conclusions; pour lui, il apparait d'abord deux feuillets blastotermiques primaires ou fondamentaux : Ie feuillet primaire externe (ectoderme) et le feuillet primaire interne (endoderme). Le premicr se différencie de bonne heure en une conche cellulaire simple (litmelle enveloppante), juis plus tard en feuillet sensoriel et en mésoderme. Le feuillet interne vient du parablaste et peutêtre concourt-il à former la lamelle vasculaire de von Baer. klein assigne aussi à l'endoderme une origine parablastique, tandis que les deux autres feuillets sont archiblastiques.

Pour Ed. Van Beneden, le germe donne naissance à la membrane enveloppante et au feuillet ectodermique destiné à se subdiviser ultéricurement en un feuillet sensoriel et en un 
feuillet moyen externe. Le parahlaste produit le feuillet endodermique destiné à former plus tard le feuillet moyen interne, qui fournira les éléments du sang, les vaisseaux et le tissu conjonclif, et à donner naissance à l'épithélium du tube digestif.

Dans un premier travail paru en 1884, Brook (25), d'après ses observations sur le Trachinus vipera, se rangeait à ma manière de voir, quant à l'origine de l'endoderme. Mais il admettait aussi que le parablaste pouvait prendre part à la formation du tube digestif. "My observations, dit-il, appear to confirm those of Henneguy, that the invagination observed in optic section in the living egg is an inward folding of the lower layer cells of the epiblast, and that aftenwards the alimentary tract is built up from this layer, together with material derived from the intermediary layer (parablaste). This point cannot, howerer, besettled definitely, without a careful examination of sections of this stage. "l'lus récemment Brook (26), après avoir étudié le développement du Motella mustela et pratiqué des coupes d'œufs de Trachinus vipera, est revenu sur sa première opinion. Pour lui, il se forme, tout autour du germe, des cellules dans le parablaste; ces cellules, plus grosses que les cellules de segmentation, viennent se placer au-dessous de l'ectoderme, pour constituer l'endoderme : "The lypoblast is not derived from the archiblast at all, but from the periblast and the yolk by a proces of segregation."

Enfin je citerai lopinion de Balfour (9), qui admet que, dans les cufs des jetits 'Téléostéens, l'endoderme provient entièrement du parablaste, tindis que chez les Salmonides ce feuillet, de inême que chez les Plagiostomes, ne serait formé que partiellement par des cellules dérivant du parablaste. Du reste Balfour n'a pas fait de recherches suivies sur ce sujet.

Mes propres observations ont porté sur la Truite, la Perche et l'Epinoche; che\% ces deux derniers Poissons, on peut suivre la réflexion du disque germinatif sur l'œuf vivant et sans l'aide d'aucun réactif; che\% la Truite ce n'est que sur des coupes que le phénomène peut être observé.

Les germes de Truite traités par l'acide osmique, puis par la liqueur de M̈̈̈ller el l'alcool, ou par le liquide piero-acétique, sont les plus favorables pour l'étude de la réflexion du blastoderme. Sur les germes durcis par l'acide chromique, la réflexion 
est beaucouy plus dilficile ì apercevoir, ce qui explique qu'elle ait été méconnue par la plupart des auteurs qui ont abopdé l'embryogénie des Salmonides, car ils ont généralement employé l'acide chromique.

Une coupe pratiquée à travers un germe traité par l'acide osmique, vers le sixième jour de l'incubation, montre nettement les diffèrentes couches qui constituent le disque germinatif. La couche superficielle, couche enveloppante, formée par une seule rangée de cellules cylindriques, en palissade, s'étend sur toute la surface du germe et s'arrête brusquement au niveau du bourrelet parablastique; c'est ainsi que la représentent la plupart des auteurs. Au-dessous de cette couche se trouve le toit de la cavité germinative, qui, dans la région médiane, est encore formé par un petit nombre de rangées de cellules. Cette seconde couche, que je désignerai dorénavant sous le nom d'ectoderme, est plus épaisse sur ses bords au niveau du bourrelet germinatif. En ce point se trouve unc troisième couche, en continuité sur le bord avec l'ectoderme, mais séparée de lui un peu plus loin par une fente très nelte qui conmunique avec la cavité germinative. Le bord du disque germinatif est arrondi et il est facile de constater en cet endroit la continuité entre l'ectoderme et la couche inférieure, que je désignerai sous le nom d'endoderme primaire. La couche enveloppante, le bourrelet parablastique et le bourrelet germinatif circonscrivent un espace triangulaire qui fait le tour du germe. Cette sorte de canal ne se voit bien que sur les germes traités par l'acide osmique; ce réactif ratatine en effet les cellules, dessine et exagère les cavités; normalement le bourrelet germinatif remplit à peu près le canal dont je viens de parłer. Les cellules marginales de la couche enveloppante sont plus développées que celles qui constituent le reste de la couche. Souvent elles donnent naissance à des cellules qui font saillie dans le canal périgermitatif et tendent à le combler. Ces cellules, peu nombreuses chez la Truite, ont été bien vues par von Kowalewski chez un Gobius, et il leur attribue un rôle important pour la formation de la vésicule de hupffer. Je n'ai pu constater leur existence chez la Truite qu'au moment de la réflexion de l'ectoderme, et il m’a été impossible de suivre leur évolution ultérieure. Je crois que leur présence indique seulement un point d'accrois- 
sement de la couche enveloppante, qui suit l'extension du blastoderme à la surface de l'ceul. Dans les pièces durcies par l'acide chromique, le canal disparaît parce que les cellules sont gontlées; il en est de même de la fente qui sépare l'ectoderme de sa portion réfléchie; cette lente est alors remplacée par une simple ligne, comme OEllacher l'a très bien représenté Planche I,

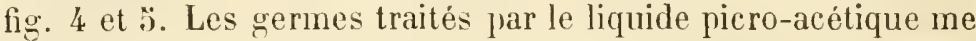
paraissent se rapprocher le plus de l'état naturel; ils montrent le canal à peine marqué, mais suffisamment cependant pour qu'on saisisse très bien la courbure du bord du disque germinatif. Quant à la fente, elle est aussi seulement indiquée et sépare nettement les deux premiers feuillets blastodermiques. La fente de séparation s'élargit vers l'intérieur de la carité germinative; là, l'endoderme primaire n'est pas nettement délimité; il est constitué par des cellules irrégulièrement disposées, dont les plus internes sont isolées et reposent sur le plancher de la cavité germinative. La partie externe de l'endoderme primaire fait suite immédiatement à l'ectoderme; au niveau de la réflexion, on trouve souvent en abondance des cellules en voie de division: ce fait prouve qu'il se produit en ce point une multiplication cellulaire très active. Au contraire, dans le parablaste sous-jacent au bourrelet germinatif, on ne voit aucun indice de formation cellulaire; les noyaux conservent leur aspect caractéristique, et le protoplasma n’est le siège d'aucun aster indiquant une organisation de cellules, comme cela sobserve ì un certain stade de la segmentation. (Planches, fig. 89.)

La partie invaginée de l'ectoderme s'étend beaucoup plus loin dans la cavité germinative du côté où se formera l'embryon que du côté opposé.

Les œufs de Perche et d'Epinoche, placés sur le côté, sous le champ du microseope, et légèrement comprimés, montrent facilement, à l’état frais, les faits que je viens de décrire chez la Truite. On peut constater sur eux l'existence réelle du canal circumgerminatif et celle de la fente qui se trouve entre l'ectoderme et sa partie réfléchie. (Planches, fig. 88.)

On doit se demander par quel mécanisme se fait la réflexion de l'ectoderme pour former l'endoderme primaire. L'extension du germe à la surface du vitellus et la formation de la cavité 
germinative ne peuvent s'expliquer que par un déplacement des éléments constitutifs du germe: c’est-ì-dire des cellules embryonnaires. Ce déplacement est produit, selon toute vraisemblance, par un mouvement amiboïde des cellules qui émigrent du centre vers la périphérie, et de la profondeur vers les parties supérieures et latérales du germe. Il n'est pas possible d'ohserver directement les changements de forme des cellules chez la Truite; peut-être pourrait-on les apercevoir dans les wufs très transparents et à développement rapide de certains Téléostéens; je n'ai rien vu de semblable dans les œufs de la Perche et de l'Épinoche, où le développement se fait trop lentement.

Un autre facteur imporlant intervient dans l'extension du germe; c'est la multiplication cellulaire. Cette multiplication se faisant principalement. dans les parties marginales du disque, on comprend que ces parties s’épaississent. A un certain moment, la migration des éléments se ralentit, tandis que la prolifération augmente dans le bourrelet germinatil. Il en résulte que les éléments nouvellement formés sont repoussés vers la cavité germinative et qu'il se produit ainsi une réflevion du blastodr pme. Cette invagination me parail être plutrit due à une prolifération cellulaire qu’à nn véritable peplojement de la eouche ertodermique; mais, quel que soit le micanisme de or processus, le résultat est le même : lá couche endodermique. primaire est formée aux dépens des cellules cetodrumiques. Tel est du moins le cas des weuĺs que j’ai étudiés, c'est-ì-dire cenx de la Truite, de la Presche et de l'Épinoche.

L'accroissement de lit conche rnveloppante ne se fait que par multiplication des cellules, el, dès qu'elle est différenciée, je ne crois pas que de nouveanx éléments viennent s'y ajouter.

Lorsque la réfle vion de l'ectoderme s'est produite au nivrau du bourrelet germinatif, lo blastoderme continue à s’étendre sur le vitellus. La migration des éléments cellulaires eontinue à se faire du centre vers la périphérie, mais l'une mamière inégale, c'est-à-dire quelle se lait plus activement vers la région embryonnaire que lu côté opprosé. Le toit de la ravité germinative s'amincit de plus en plus et finit par être réduit ì une seule couche de cellules, recouverte toujours par la couche enveloppante, dont les cellules sont devenues alors pavimenteuses. 
L'ectoderme s’épaissit au niveau de l'écusson embryonnaire, et l'entloderme primaire s'étend de plus en plus au-dessous de lui. dans la cavité germinative. Ce dernier feuillet s'épaissit également dans sa portion initiale. Sur le reste du bourrelet germinatif les deux feuillets restent au contraire assez minces, formés sculement de deux ou trois couches de cellules. et l'endoderme ne s'étend que peu dans la cavité germinative.

La cavité germinative, en s'agrandissant par suite de l'cxtension du germe sur le vitellus. diminue de hauteur. de telle sorte que son toit est presque en contact arec le parablaste, dont il est cependant séparé par une petite quantité de liquirle.

Liextension du parablaste suit celle du germe; celte couche présente son maximum d'épaisseur au-dessous de l'écusson embryonnaire; c'est anssi en ce point que les noyaux qu'elle renferme sont les plus nombreux.

Le mésoderme ne commençant à apparaitre qu assez tardivemont, lorsque l'embryon est déjà différencié à la surface du blastoderme, je ne moceuperai du développement de ce feuillet qu'à propos de l'évolution de l'embryon.

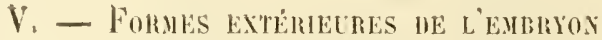

Les formes extérieures de l'embryon des Salmonides ont été très birn étudićes rar plusieurs auteurs, entre autres par OAllarher (123), lis (85), liupffer (106) et Goronowitsch (57).

OEllacher a établi, pour la Truite, un certain nombre de stades du développement de l'embryon auxquels il a donné des noms particuliers et qui correspondent à certains jours de l'incubation. Halheureuscment la durée de l"incubation étant très variable, ainsi que je l'ai déjà dit, il est impossible de rapporter chacun des types d'OEllacher it un nombre constant de jours depuis le moment de la fécondation. Dans la description des coupes sur lesquelles jai étudié le développement de la Truite, je désignerai les cmbryons d'après les stades établis par OEllacher, stades dont jai pu vérifier l'exactitude. Hais, pour simplifier les appellations d'OEllacher et aussi pour multiplier les stades du développement embryonnaire, je désignerai. comme Ballour (8) l’a fait dans sa belle monographie de l'embryologie 
des Blismobranches, elatque stade par une lethe de l'alphahet. Ces stades représcontés figures 7 à 1 ' et figures 47 à :ig (Planclies), ont été dessinés ì la chambre claire d'après des pièces durcies par l'iuda chromiqur.

Stane A. Planches, fig. 1.3 et fig. 4.7.) - Nous avons laissé le germe segmenté an moment où les bords du blastodisque šinfléchisseut dans la cavité sons-germinative pour constituer le feuillet intrine frimaire. La première apparition de l'embryon consiste dans un épaississement du bourrelet marginal, en un point de son bord interne: à ce niveau, le bonrrelet est plus large que dans le reste de son étendue. Cette portion élargie est mal délimitée et comprend à peu près le quart de la circonférence du germe. Ce stade correspond au rudiment $\mathrm{cm}$ bryonnaire primitif (primitive Embryonalanlage) d'oEllacher.

Stane B. (Planches, fig. I't el fig. 48.) - La partio renflée du bourrelet blastodermique est devenuc plus apparente; alle est nettement arrondic dans sa portion médiane et forme une saillic, beaucoup plus marquéc qu'au stade précédent, vers lat partie centrale du blistoderme. Sur la plupart des oufs, elle se présente comme une masse lomogène mal délimitér, allssi bien sur ses parties latérales, qui se continuent avec le bourrelel blastodermique, qu’à sa partie antérieure, c’est-ì-dire dans sa portion opposée au bourrelet. Sur certains œufs, la première ébauche de l'embryon parait formée de denx masses accolées au bourrelet blastodernique el séparées l'une de l'autre par une légère dépression, plus marquée et plus large en avant qu'en arrière. Get état correspond, je crois, à un stade un peu plus arancé du díveloppement, et montre le débul de l'apparition du sillon mélullaire qui devient très visible au stade suivant. Dans l'axe de la dépression, qui rst l'are longiturlinal du fulur embryon, commence à se former, sur le bord esterne du disque blastodermique, une petite saillie qui ast le bourgeon caudul d'OEllacher, ou le bourgeon marginal de llis, le bourgeon final de liupffer, la proéminence cuzdale de Ballour (Planches, fig. $48, b c)$. Je lui conserverai le nom de bouryeon caudal parce que éest le plus ancien, bien que ce termr soil impropre, car ce bourgeon ne constitue par la queue de l'embryon. 
OEllacher a désigné ce stade sous le nom d'écusson embryonuare arrondi (runde Embryonalschild).

Stane C. (Planches, fig. 40 et fig. 49 et 50.$)$ - L'écusson embryomaire est plus apparent et mieux délimiti: il présente une partie légèrement rétrécie ì son point dinsertion sur le hourrelel blastortermique; sa forme est à peu près oviale. De son extrimité antérieure partent deux bandes plus claires qui Je rattachent an bord interne du bonrrelet marginal. La petite saillie du bord externe du bourrelet blastodermique, siture dins l’ave de l'embryon, est plus accentuée et représente alors nin veritable petit bourgeon (Planches, fig. 49, bc).

la partic axiale de l'embryon est occupéc par une dépression en forme de $V$, dont la pointe est en avant du bourgeon caudal, et dont l'ouverture correspond ì l'extrémité antéricure de l'rmbryon. Cette dépression est la promière ćbauche de la gouttière médullaire; elli est limitér des deux còtés par deux bonrrelets silllants qui forment les branches du V. Cette disposition est constate, mais plus on moins nette suivant les embryons. Quelquefois, te sillon est à peine marqué (Planches, fig. 49). landis que chez d'autres embryons il est largement ouvert en avant (Planches, fig. :30).

llis (85) parait itro le premier qui ait entresu une forme semblable de l'embryon chez le Samon. La figure 3 quil donue dans sou ménoire di 1878, p. 184, ressemble à celle que nous figurons de ce state chez la Truite. Copendant, daprès His, les dem bords du sillon mólullaire se rejoindraient en avant pour former une sorte de fer à cheval. Il est probable que l'embryon que cet anteur a figuré correspond ì un starle un peu plus avané. Chaz la Truite, les hords du sillon médullaire se lapprochent en effet par lenr partice antérieure entre le stade $\mathrm{C}$ et le stade D. Ziegler 200 (Pl. HI, fig. t) al representé un embryon de Simmon identique à cems que jai observes à ce starle.

(Willateher a domné à ce stade le nom d'écusson cmbryonuaire oralaire transeresal (querovale Embryomalschild); la figure quïl "n donne montr" bien le commencenent du sillon medullaire, mais soms forme diun simple dépression linéaire. Il in-

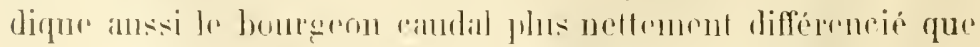


je ne l'ai vu et séparé du r'este de l'embryon par une dépression semi-circulaire.

Stade D. (Planches. fig, 16 et 17 , et fig. 81 et 82.) - Le blastoderme mesure environ $3^{\mathrm{mm}}, 70$ de diamètre. L'écusson embryonnaire s'est allongé dans le sens antéro-postérieur et rétréci latéralement. Il est devenu piriforme et sa longueur est de 1 millimètre. Le sillon médian est beaucoup plus étroit quau stade précédent, mais aussi plus profond et plus nettement délimité. La partie antérieure de ce sillon forme une fossette ovale très allongée, plus profonde et plus large que la partie postérienre, qui est très superficielle et à peine indiquée. La partie antérieure de l'embryon représente assez bien un fer à cheval, qui embrasse le sillon médullaire par sa concavité; de chaque extrémité du fer à cheval partent deux replis, mal délimités, qui vont rejoindre la partie interne du bourrelet blastodermique.

Ce stade a été assez bien représenté par OEllacher et par His. OEllacher le désigue sous le nom d'écusson embryonnaire piriforme (birnfœrmige Embryonalschild); la figure qu'il en donne diffère de celle qui est représentée figure 51 (Planches), en ce que les contours de l'écusson ne présentent pas la petite encoche qu'on remarque de charue cóté, vers lo milieu de la longueur de l'embryon, au point où se détaclent les replis qui ront rejoindre le bourrelet blastndermique. De plus, dams la figure d'Otllather. te bourrelet blastodermique ne parail ètre en rapport quavec le bourgeon caudal et présente partout la mème largeur. Les encoches latérales de l'embryon ne sont pas, en effet, toujours visibles, mais je les ai observées assez souvent pour pouvoir les regarder comme normales. Je ne sais au juste quelle est leur signification; je crois cependant qu'on doit considérer les deux saillies qui déterminent ees encoches comme les premières ébauches des vésicules optiques. Quant à l'élargissement du bourrelet de chaque côté de l'embryon (Planches, fig. 52, be), on l'observe toujours; e'est ce que Kupffer (106) et OEllacher désignent sous le nom de marge ou bordure embryonnaire (Embryonalsaum), et qu'Osllacher a figuré au stade B et aux stades E et F.

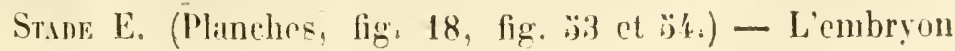


s'est allongé davantage et mesure environ $1^{\mathrm{mm}}, 7$. Le sillon longitudinal superfieiel s'est allongé en même temps. Vers le quart antérieur de l'embryon, on remarque un petit sillon transversal, et en arrière de lui un autre sillon plus marqué et plus étendu, qui se trouve an tiers de la longueur totale de l'embryon; enfin une troisième petite dépression à peine marquée se trouve à l'union du tiers postérieur avec les deux tiers antérieurs de l'embryon.

Le bourgeon caudal est plus développé qu'au stade D; ses contours sont mieux délimités. De chaque côté de l'embryon, depuis le niveau du sillon transversal moyen jusqu'an bourrelet blastodermique s'étend une lame en forme de croissant, qui est la bordure embryonnaire (Planches, fig. $53 \mathrm{bc}$ ).

L'embryon en forme de lancette (laneetformige Embryonalschild), qu'OEllacher a représenté Planche 1, fig. 10, correspond à notre stade E. Cette figure, comparée à la nôtre, n'offre que quelques légères différences. Dans la figure de l'auteur allemand, le premier sillon transversil antérieur est moins rapproché dı sillon moyen que sur notre figure, et la dépression postérieure n'existe pas; de plus, le sillon longitudinal s'avance davantage vers l'extrémité céphalique et se termine par une petite dilatation plus marquée que sur notre embryon. OAllaclıer considère cette dilatation antérieure et les deux sillons transversaux qui la suivent, comme les jremières ébauches des trois vésieules cérébrales; le plus grand de ces sillons présente à ses extrémités une petite fossette qui est l'origine de la vésicule auditive. Cette interprétation est évidemment exacte, étant donnée la situation quoceuperont plus tard ces différents organes, mais on ne doit pas attacher une grande importance ì la configuration extérieure de l'embryon, aux premiers stades du développenent. Comme te fait, en effet, très bien remarquer OEllacher, les sillons transversaux et les fossettes ne sont pas toujours bien visibles et ne présentent pas une forme constante ni une place bien déterminée, sur des embryons exactement du même âge. Les coupes transversales et longitudinales de ces embryons montrent que les dépressions dont il s'igit sont tout à fait superficielles, et qu'il ne se produit à leur niveau aucune invagination comparable ì celle qui sobserve chez les autres Vertébrés. Les dépressions, aussi bien Je sillon longiturlinal 
que les sillons transversaux, disparaissent presque complètement sur des embryons fixés par le liquide de Kleinenberg; on ne les voit bien que sur les embryons durcis par l'acide chromique. Les élénents embryonnaires sont constamment en voie de prolifération, principalement dans les régions qui s'accroissent le plus rapidement; l'axe nerveux est dans ce cas, surtout au starle que nous considérons. Or les cellules en cytodiérèse sont beaucoup plus sensibles que les autres à l'action des réactifs. Le liquide de Kleinenberg, par exemple, les gonfle; l'acide chromique les désorganise souvent. Il en résulte que dans les points où la cytodiérèse est active, on observera tantôt une augmentation, tantòt une diminution de volume, suivant le réactif fixateur employé. Avec le liquide de Kleinenberg, les dépressions superficielles de l'embryon s'effacent par suite du gonflement des cellules sous-jacentes; avec l'acide chromique, elles s'exagèrent par suite de la destruction et l'écrasement de ces mèmes cellules. On comprend done comment, aver lacile chromique, on peut observer des diffẻrences de forme et de position des dépressions superficielles, suivant que les éléments embryonmaires auront présenté une cytodiérèse plus ou moins active dans telle ou telle région, au moment de la fixation.

Quoi qu'il en soit. on vloit considérer, avec OEllacher, les dépressions superficielles de' l'embryon, fossettes et sillons transversaux, comme indiquant la place des trois vésicules cérébrales types des embryons des autres Vertébrés.

En embryon du stade E, détaché avec soin du vitellus, coloré par le carmin, et nonté dans le baume dı Ciınada, est représenté, vu par transparence (Plinches), fig. :34. On apercoit sur la ligne médiane une partic plus foncéc présentant une dilatation dans la région antérieure, et une autre dilatation noins marquéc dians te bourgeon caudal. Cette région médiane obscure est la partie la plus épaisse de l'embryon, l'axe nerveux, qui s'enfonce comme une carène dans le vitellus. Le renflement antéricur correspond à la partie comprise entre l'extrémité céphalique et le sillon des vésicules auditives (Planches, fig. $54 \mathrm{c}$ ).

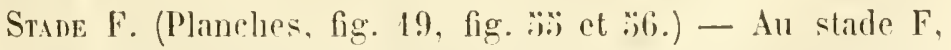
10. blastodorme a recouvert à pen près la moilié du vitulhus: lo 
bord externe du bourrelet blastodermique est très voisin de l'équateur de l'œuf. L'embrỵon mesure environ $2^{\mathrm{mm}}, \ddot{3}$; il est plus étroit qu au stade E, principalement dans la région céphalique. Le sillon médullaire est beaucoup moins apparent qu'au stade précédent. Le sillon transversal des vésicules auditives est devenu moins visible; en arrière de lui on voit deur ou trois fossettes médianes qui sont des restes du sillon métlullaire, en voie deffacement. Les deux moiliés du corps de l'embryon semblent s'écarter en avant du bourgeon candal, de manière a former un angle dans lequel le bourgeon caudal est compris. Cet écartement n'est qu'une simple apparence due à l'existence d'une légère dépression tout à fait superfieielle, en avant du bourgeon caudal (Planches, fig:

Les contours de l'embryon présentent, au niveau du sillon des vésicules auditives, un léger rétrécissement et un autre un peu plus bas, vers le milieu de la longueur totale de l'embryon. La bordure embryonnaire est beaucoup moins large qu’au stade E; elle tend à s’effacer et remonte jusqu'à la partie la plus large de la région céphalique.

Le stade $F$ correspond an stade figuré par OEllacher, fig. 12. Cet auteur décrit un stade d'embryon en forme de fer de lance (lanzenspitzfœrmige Enbryo) intermédiaire entre E et F, dans lequel la partie la plus large se trouve au niveau de la troisième vésicule cérébrale, et dont la parlie postérieure semble ètre profondément engagée dans le bourrelet blastodernique, très épaissi en ce point. OEllacher reconnait lui-même que la fiğure qu’il donne n'est pas facile à comprendre, et il pense que l'enbryon doit son aspect partieulier à ce qu’à ee stade il est enfoncé dans le vitellus et quion ne roit que sa partie supérieure. Je n'ai pas observé d'embryons ayant l'aspect représenté par OEllaclier et je crois que cet anteur aura eu affaire à des embryons déformés par la compression exercée sur le cliorion par la masse vitelline, lors de sa coagulation.

Un embryon du stade $F$, coloré et examiné par transparence dans le baume du Canada (Planches, fig. „36), offre le plus grand intérêt. De même qu'au stade précédent, il existe sur la ligne médiane une région obscure correspondant à la carène de l'axe nrrveux. Cette carène n'est pas droite, elle présente, dans la région céphalique, des sinuosités qui paraissent être dues à un 
tassement qui se serait produit lors de la fixation de l'embryon, par suite d'une contraction de ce dernier. De chaque côté de l'axe, dans la partie antérieure de la moitié postérieure de lembryon, on voit einq à six masses rectangulaires qui sont les premières protovertèbres. L'apparition de ces organes est très importante ì noter, car, dès Jors, il devient facile de distinguer nettement la région céphalique de l'embryon, du trone qui commence au niveau de la première protovertèbre.

Stane F'. (Planches, fig. :57.) - A la fin du stade F, l'embryon subit quelques modifications dans sa forme extérieure. Mais la structure interne n'ayant pas éprouvé de grands changements, je n'ai pas voulu faire un stade particulier de celte forme, le considérant seulement comme la fin ilu stade F.

Le sillon médullaire et les dépressions superficielles ont entièrement disparu. Le sillon médullaire est remplacé par un cordon saillant qui s'étend depuis la tête jusquau bourgeon caudal, en se bifurquant ì ce niveau, comme au stade précédent. La région cépllalique est encore amincie et a changé de forme; l'extrémité antérieure est maintenant la plus large et présente deux renflements latéraux qui sont les vésicules optiques, puis vient une partie retrécie suivie d'une nouvelle dilatation au niveau des vésicules autidives. A partir de ce point, jusqu'au bourrelet blastodermique, s'étend de chaque côté de l'axe nerveux une bande étroite, moins saillante que l'axe, c'est la région des protovertèbres, qui ne sont pas encore visibles extérieurement. En dehors de cette région se trouve la bordure embryonnaire qui se rétrécit de plus en plus (Planches, fig. 57).

OEllacher a représenté, figure 13, un embryon correspondant à peu près à ce stade, mais dont l'aspect est un peu différent. Dans cet embryon il existe, sur la ligne médiane de la région céphalique, une saillie qui se continue avec celle de l'ixe médullaire. De clıaque côté de la tète, les vésicules auditives se montrent sous la forme de deux éminenees réniformes, accolées sur la ligne médiane par leur partic convexe. Cette configuration doit faire admettre que l'embryon d'OEllacher est un peu plus àgé que celui qui correspond à notre stade F'.

Stape G. (Planches, fig. 20 et 21, fig. 58.) - L'embryon 
mesure 3 millimètres. Certains de ses organes sont devenus visibles extérieurement. Sur toute sil longueur, la lig̨ne médiane est occupée par une saillie formée par la partie centrale de l'axe nerveux, saillie qui, au stade $\mathrm{F}^{\prime}$, n'ètait apparente que dans la région du trone. Les vésicules optiques se détachant nettement de chaque eòté de l'axe neédian sous forme de masses hémisphériques. Les vésicules auditives se montrent également. ainsi qu'OEllacher les a représentées, comme deux masses réniformes à convexité interne. En arrière de ces vésicules commence la région protovertébrale, comprenant environ douze somites, dont chaque moitié apparaît sous forme d'un petit rectangle, séparé de ses voisins par une petite dépression. La région postérieure de l'embryon n'a pas subi de changements (Planches, fig. 58).

La figure 14 d'OEllacher est celle d'un embryon arrivé au stade G. L'auteur signale une disposition particulière des protovertèbres qui ne sont pas parallèles entre elles. Suivant lui, les protovertèbres antérieures ont leur axe dirigé d'avant en arrière et de dehors en dedans; les moyennes ont leur axe perpendiculaire à l'axe du corps et les postérieures sont dirigées d'arrière en avant et de dehors en dedans. Je n'ai pu observer une semblable disposition qu'accidentellenent, et j'ai presque toujours vu les protovertèbres parallèles entre elles et dirigées perpendiculairement à l'axe du corps.

Stane H. (Planches, fig. 22 et 23, et fig. 59.) - La forme de l'embryon s'est à peine modifiée depuis le stade précédent, sa longueur est de $3^{\mathrm{mm}}, 60$. Les vésicules optiques sont plus développées, le cristallin commence à apparaître, les protovertèbres ont augmenté de nombre. La partie postérieure de l'embryon est la plusintéressante à considérer. Le blastoderme a recouvert presque entièrement le vitellus et le bourrelet est réduit à un simple anneau elliptique, placé à l'extrémité de l'embryon. Le bourgeon caudal n'est plus visible et le grand axe de l'anneau blastodermique est dans le prolongement de l'axe du corps. Ainsi que l'ontremarqué tous les auteurs, la fermeture du blastoderme a lieu toujours sous forme d'une ellipse plus ou moins allongée, suivant les sujets, et qui finit par se réduịre à une fente linéaire dont les bords se rapprochent et se 
soudent. La fermeture a lieu à une période de développement variable dans des œufs de même àge. Le nombre des somites virie, en effet, chez les embryons de la fin du stade II, de 18 à 26 (Planclies, fig. :99).

La description que je viens de donner des formes extérieures de l'embryon, aux différents stades, est à peu près conforme, ainsi qu'on a pu le voir, à celle qui a été faite par OAllacher. Les figures de Goronowitsch $\mathbf{5 7}$ ) ressemblent aussi beaucoup à celles d'OEllacher et aux miennes, mais l'interprétation qu'il en donne diffère sur quelques points. Pour lui, il existe toujours entre la région eéphalique et le bourgeon caudal, un trone rudimentaire, mème aux stades les plus précoces. ll est difficile de se prononeer sur la valeur de cette assertion, car aux stades C et D, l'embryon est encore trop peu développé pour qu'on puisse dire si l'écusson embryonnaire, qui est en rapport avec le bourrelet, représente uniquement la tête ou celle-ci avec une portion du trone. Goronowitsch admet que les deux premières fossettes qui apparaissent sur le trajet du sillon médullaire représentent le cerveau antérieur et le cerveau postérieur; le cerveau moyen n'apparaitrait que plus tard entre les deux. Pour nous, les trois fossettes, correspondant aux trois vésicules cérébrales primaires, apparaissent en même temps.

Bien différentes des figures d'OEllacher, de Goronowitsch et des miennes, sont celles de Kupffer (106), publiées, en 1884, dans la première partie d’un travail dont la suite n’a pas encore paru. Les notions fournies par l'étude de la structure interne de l'embryon étant indispensables pour discuter les figures de Kupffer et l’interprétation quil en donne, je n’indiquerai la manière de voir de cet auteur qu'en traitant le développement du système nerveux.

\section{VI. - Étude nes coupes des différents stanes.}

L'étude de la forme cxtérieure de l'embryon de la Truite, aux différents stades de son développement, n'apprend pas grand'chose sur l'évolution des parties essentielles, c'est-ádire sur la formation des divers systèmes. Les coupes transversales et longitudinales, faites à travers les jeunes embryons, sont au contraire des plus instructives. Je décrimai d'abord 
laspect de ces coupes aux differents stades que jat chtablis, et jexposerai ensuite le déroloppement de chaque système en particulier.

Stane A. - Je ne reviendrai pas iei sur ce stade dont j’ai décrit les coupes à propos de la formation du blastoderme.

Strne B. - Une coupe longitudinale passant par l'axe de l'ébauche embryonnaire montre ì peu près la même disposition qu'au stade précédent. L'ectoderme se continue à la périphérie avec l'endoderme primaire. Les deux feuillets ont à peu près la même épaisseur; cependant l'eetoderme est un peu plus développé que l'endoderme et présente son maximum d'épaisseur dans lá région postérieure de l'écusson embryonnaire. Le feuillet supérieur se continue antérieurement avec le toit de la eavité germinative formé par une seule rangée de cellules; il est recouvert sur toute son étendue par la lame enveloppante. L'endoderme primaire se termine antéricurement à la limite de l'écusson embryonnaire; quelques cellules se détachent de son extrémité et se trouvent libres ou en petits groupes sur le plancher de la cavité germinative.

Les coupes longitudinales parallèles ì la médiane, et intéressant les bords de l'écusson embryomaire, montrent la même disposition que la précédente, mais on voit que, sur les bords latéraux de l'éeusson, les feuillets sont beaucoup plus minces et que l'endoderme s'étend beaucoup moins loin.

Sur des coupes transversales du même stade, passant par l'extrénité postérieure de l'embryon, c'est-à-dire au niveau du bord externe du bourrelet marginal, le blastoderme se présente sous la forme d'une lentille biconvexe. En ce point on ne distingue aucun feuillet; au centre de la coupe, sur la ligne médiane, on observe une disposition très curieuse des éléments embryonnaires, disposition signalée par OEllacher (123) et sur laquelle les autres auteurs n’ont pas insisté. Les cellules sont groupées en ce point en un amas arrondi; elles sont disposées en cercles concentriques autour d'un centre formé par trois ou quatre cellules. Le nombre des cercles est de trois ou quatre; les cellules ainsi disposées sont allongées et leur grand axe est perpendiculaire au rayon du cerele. L'imas 
cellulaire, qui oceupe ainsi l'axe de la partie postérieure de l'embryon est le cordon axiul (Axenstrang) d'OEllacher. II n'eviste, à ce moment, que dans la partie de l'écusson embryonnaire comprise dans le bourrelet marginal; plus tard, il s’étend plus en avant (Planclıes, fig. 90).

Les coupes transversales plus antérieures présentent les deux l'euillels primaires séparés par une ligne cliire représentant une fente virtuelle, reste de lit eavité germinative. Cefte fente s’arrète à une certaine distance des hords de l'écusson embryonnaire; sur les bords, en effet, les deux feuillets sont en continuité l'un avec l'autre. A mesure qu'on se rapproche de l'extrémité antérieure de l'écusson, l'endoderme diminue d'épaisseur et finit par être remplacé par des groupes isolés de cellules, mais on le retrouve toujours dans les parties latérales de la coupe, au niveau du bourrelet marginal. Enfin, en avant de l'écusson, l'ectoderme, réduit à une simple couche de cellules, recouvre seul la cavité germinative : il s'épaissit latéralement, se réfléchit et constitue l'endoderme primaire du bourrelet marginal. L'aspect des coupes transversales de la partie extra-embryonnaire du blastoderme reste le même aux stades suivants; les coupes augmentent de plus en plus en diamètre à mesure que le blastoderme s'étend sur le vitellus, mais la constitution du toit de la cavité germinative et celle du bourrelet marginal ne changent pas.

Stade C. - Les coupes longitudinales de ce stade sont à peu près identiques à celles du stade précédent; elles montrent cependant l'extension des deux premiers feuillets vers le centre de la cavité germinative; celle-ci diminue de hauteur; son toit tend à s'appliquer sur le plancher (Planches, fig. 75 ).

Les coupes transversales fournissent plus de renseignements que les coupes longitudinales. Au niveau du bourgeon caudal, on retrouve le cordon axial (Planches, fig. 90); ce cordon existe aussi à la partie postérieure de l'embryon, là où les deux premiers feuillets sont différenciés et séparés par une ligne correspondant à une fente virtuelle. Cette fente traverse le cordon axial qu'elle coupe en deux parties égales, de sorte qu'une des moitiés est contenue dans l'ectoderme, l'autre dans l'endoderme primaire (Planches, fig. 91). Cette disposition a échappé à OElla- 
cher, qui a représenté les deux feuillets unis au niveau du corlon axial. La ligne de séparation est en effet assez difficile à voir; ce qui frappe surtout au premier examen de la coupe, c'est la disposition des cellules en cercles concentriques, et il est très intéressant de voir cet arrangement se correspondre dans chacun des deux feuillets.

Une série de coupes transversales, pratiquées d'arrière en avant, permet de suivre les variations d'épaisseur des feuillets blastodermiques (Planches, fig. 65 à 74 ).

Le sillon longitudinal médian, qui se voit très bien sur les vues en surface et qui est la première ébauche du système nerveux, est beaucoup moins net sur les coupes. Celles-ci présentent cependant dans leur partie médiane une dépression correspondant au sillon; cette dépression s'élargit et tend à s'effacer d'arrière en avant. L'ectoderme, en avant du bourgeon caudal, est plus épais que l'endoderme primaire et présente son maximum d'épaisseur sur la ligne médiane; il va en s'amincissant vers la partie antérieure de l'écusson embryonnaire. Sa plus grande épaisseur est alors de chaque côté du sillon neural, et son minimum d'épaisseur est au fond du sillon. L'endoderme est au contraire plus épais sur la ligne médiane de l'embryon, mais, à la partie antérieure, il s'amincit et finit par disparaître à l'extrémité du sillon neural; en ce point, l'endoderme n'existe plus que sur les parties latérales de l'embryon.

Stade D. - Ce stade est un des plus intéressants; c'est en effet à ce moment qu'apparait la corde dorsale et que se forme le feuillet moyen. Sur une coupe longitudinale médiane, on voit, en avant du bourgeon caudal, l'endoderme primaire se diviser en deux couches, dont l'une supérieure comprend presque toute l'épaisseur de ce feuillet, tandis que la couche inférieure est constituée par une ou deux rangées de cellules seulement. Cette division de l'endoderme primaire ne s'étend pas très loin, elle cesse d'être visible dans les deux tiers antérieurs de l'embryon. Les cellules de la couche supérieure se sont allongées dans le sens vertical et pressées les unes contre les autres dans le sens antéro-postérieur. Cette couche supérieure est l'ébauche de la corde dorsale. Les cellules de la couche inférieure, qui forme l'endoderme proprement dit, conservent leur aspect arrondi ou 
polyédrique, par pression réciproque. L'ectoderme prend un grand développement dans la région antérieure de l'embryou; mais à l'extrémité du renflement céphalique, il se termine en pointe et il est dépassé à ce niveau par l'endoderme qui est plus épais que lui (Planches, fig. 81).

Les coupes longitudinales intéressant les parties latérales de l'embryon, montrent que la division de l'endoderme primaire s'est effectuée aussi dans ces rúgions. Mais la couche supérieure, qui représente le mésoderme, est bien moins épaisse que sur lit ligne médiane, et les cellules ne présentent pas la disposition qu'elles offrent dans l'ébauche de la corde dorsale (l'lanches, fig. 82).

Si l'on examine les coupes transversales correspondant à celles que je viens de décrire, on voit qu'au niveau du bourgeon caudal rien n`a changé depuis le stade précédent. Immédiatement au-devant de lui on ne tronve encore que les deux feuillets primaires. Le corton axial est divisé par la ligne de séparation des feuillets, mais il est divisé d'une laçon asymétrique. L'ectoderme est ì ce niveau plus épais sur la ligne médiane que sur les parties latérales; il renferme la plus grande partie du cordon axial. Lat portion inférieure de ce dernier est contenue dans l'endoderme primirire; elle s'en sépare par une ligne très nette, surtout bien marquée dans les préparations traitées par l'acide osmique. Le demi-eylindre qui résulte de cette différenciation de li partie inférieure du cordon axial est la corde dorsale (Planches, fig. 77, fig. 93 et fig. 110).

Sur une coupe un pen plus antérieure, il existe de chaque còté de la corde dorsale une ligne elaire qui, dans les préparations à l'acide osmique, apparait sous forme d'une fente; elle sépare l'endoderme primaire en deux couches, dont la supérieure est le mésoderne et l'inférieure l'endoderme définitif. La corde dorsale est encore plongée dans l'endoderme dont elle n’est séparée que par une ligne claire (Planclıes, fig. 78).

Dans la partie antérieure de l'embryon, on ne trouve plus que l'ectoderme et l'endoderme primaire (Planches, fig. 79 et 80); ils présentent sur la ligne médiane la disposition concentrique des cellules, disposition qui s'observe du reste dans toutela longueur de l'embryon. Mais, dans l'extrémité antérieure, les cercles concentriques deviennent des ellipses dont le grand axe 
correspond à la ligne de séparation des deux feuillets. A ce niveau, l'endoderme est plus développé que l'ectoderme, surtout sur la ligne médiane.

Stade E. - L'embryon s'est aceru en longueur. Les coupes longitudinales ont cependant à peu près le même aspect qu'au stade D. L'ectoderme prend, dans la région antéricure qui correspond au cerveau, un développement plus considérable; l'endoderme primaire diminue au contraire d'épaisseur, sauf à l'extrémité tout à fait antérieure, où il demeure encore plus épais que l'ectoderme. La corde dorsale s'étend un peu plus loin qu'au stade précédent, et ses cellules sont mieux différenciées.

A l'extrémité postérieure de l'embryon, au point où la corde dorsale se dégage du bourgeon caudal en même temps que l'ectoderme et l'endoderme, les cellules endodermiques présentent un aspect particulier. Elles sont plus grosses que les autres : quelques-unes offrent des figures cytodiérétiques; elles forment, en contact avec le parablaste, une rangée d'une dizaine cnviron d'éléments cylindriques. Cet amas cellulaire est l'origine d'un organe important qui, aux stades suivants, devient beaucoup plus apparent, sous forme d'une vésicule creuse, et que j'ai signalée pour la première fois chez la Truite, en 1880 (69). Par sa position, cette vésicule est identique à celle que Kupffer a signalée chez l'Épinoche et qu'il a désignée sous le nom impropre d'allantoïde. Kupffer a eu le mérite d'attirer l'attention sur la vésicule qui se trouve à l'extrémité postéricure de l'embryon de certiins Poissons osseux, aussi lui donnerai-je, à l'exemple de la plupart des auteurs, le nom de vésicule de liupffer; mais cet organe avait déjà été vu avant lui par Coste, qui l'a très bien représenté dans l'atlas de son grand ouvrage (Histoire générale et particulière du développement des corps organisés), figure 8' de la Planche 11 relative au développement de l'Epinoche (1). Depuis Kupffer, la vésicule a eté observée chez un grand nombre d'espèces, par plusieurs auteurs; elle n'avait pas encore été signalée chez les Salmonides. OEllacher (123) n'en parle pas dans son beau mémoire sur le développement de la Truite. C.-K. Hoffmann (89) ne l'a

(1) Coste ne fait aucune mention de la vésicule dans l'explication de la figure. 
pas figurée. Ziegler (200) l’a décrite récemment chez le Saumon. Je signalerai au stade suivant les caractères qui distinguent lia vésiculc de la Truite.

Les coupes transversales du stade E aceusent une différenciation plus nette de la corde dorsale et des lames mésodermiques, qui sont alors bien séparées de l'endoderme secondaire. Mais les changements survenus depuis le stade D ne sont pas assez importants pour qu'il soit nécessaire d'y insister.

Stane F. - Sur une coupe longitudinale médiane, la vésicule de Kupffer se voit très nettement à l'extrémité antéricure du bourgeon caudal, là où au stade précédent existait la rangée de cellules endodermiques plus grosses, que j’ai décrite. La vésicule est maintenant bien formée; elle mesure environ $0^{\mathrm{mm}}, 11$ de diamètre longitudinal; sa forme est celle d'un demi-cercle, dont le diamètre est parallèle au vitellus ef dont l'arc regarde la partie dorsale de l'embryon. Ses parois sont constituées par une seule rangée de cellules cylindriques; celles du plancher sont plus courtes el moins régulièrement disposées (Planches, fig. 108 k). En avant de la vésicule, l'endoderme se continue, comme au stade précédent, jusqu'à l'extrémité de l'embryon (Planches, fig. ๖3).

Les coupes longitudinales, parallèles à la ligne médiane et passant en dehors de la corde dorsale, montrent que, dans la région mojenne de l'embryon, le mésoderme est divisé en un certain nombre de masses cellulaires de forme rectangulaire, et placées les unes à còté des autres. Le grand axe des rectangles est perpendiculaire à la surface vitelline; dans chacun d'eux les cellules périphériques sont régulièrement disposées, les cellules centrales sont au contraire irrégulièrement agencées. Chacune de ces masses cellulaires, au nombre de trois à six de chaque côtẻ de la ligne médiane, représente un somite (Planches, fig. 84).

Les coupes transversales du stade $\mathrm{F}$ sont plus instructives que les coupes longitudinales, au point de vue du développements des feuillets (fig. 1 ì 11).

A la partie tout à fait antérieure de l'embryon, on ne trouve encore que les deux feuillets primaires. L'ectoderme, dont la section est piriforme, pénètre conme un coin dans l'endoderme 
primaire qui se trouve refoulé sur les còtés (fig. 1). Plus en arrière, au niveau de la prenière vésicule cérébrale, l'ectoderme est beaucoup plus développé: son épaississement médian présente deux renflements latéraus arrondis qui sont les ébauches des vésicules oculaires. Liendoderme primaire, sur la ligne médiane, est en contact direct avec la carène de la masse nerveuse. Hais latéralement ce fruillet s'est différencié en deux couches, une couche mésodermique qui n'cxiste qu'au-dessous des vésicules optiques, et une couche endodermique, en rapport avec le parablaste et en rapport direet avec l'ectoderme, sur les parties latérales de l'embryon. Entre le mésoderme et l'angle interne inférieure de la vésicule optique se trouve une petite cavité à section triangulaire (fig. 2 et 3 ).

Les coupes suivantes montrent la masse nerveuse plus développér qu’au niveau des résicules optiques, et présentant une forme de triangle isocèle allongé, dont la base convexe, dirigće en haut, se continue latéralement avec la couche ectodermique. Le mésoderme sépare complitement l'ectoderne de l'endoderme; ce dernier est moins étendu que dans la région tout à fait antérieure, et le mésoderme le déborde latéralement (fig. 4. et :3).

Le point où finit la région céphalique rst marqué par l'apparition de la corde dorsale sur la région médiane el all-rlessous de lave nerveux. A re niveall, la masse nerreuse présentr latéralement, comme dans la rógion oculaire, deus renflements eetodermiques qui sont les rudiments des vésicules aulitives: ces renflements sont en continuité par leur base avec la partic supérirure de l'axe nerveus et en sont séparés latéralenent par une fente virtuelle (fig. (i) (Planches, fig. 9.).).

En arrière de la tête, les coupes conscrvent le méme aspect jusque dans le voisinage ilu bourgeon caudal. L'axe nerveux est heaucoup plus étalé el noins rléveloppé que dans la région céphalique. Les lames mésorlermiques présentent, sur certaines coupes, les masses protovertébrales de chaque còté de l'axe nerveux et, latéralement, elles sont divisées longitudinalement par une fente virtuelle, qui est le futur cœlome (fig. 7 et 8).

A mesure quon se rapproehe du bourgeon caudal, laxe nerveux devient de plus en plus petit, et les masses latérales mésodermiques prennent au contraire une plus grande importance. 
En mème temps, la carène de l'axe nerveux s'allonge dans le sens vertical, et sa délimitation d’avec la corde dorsale est de

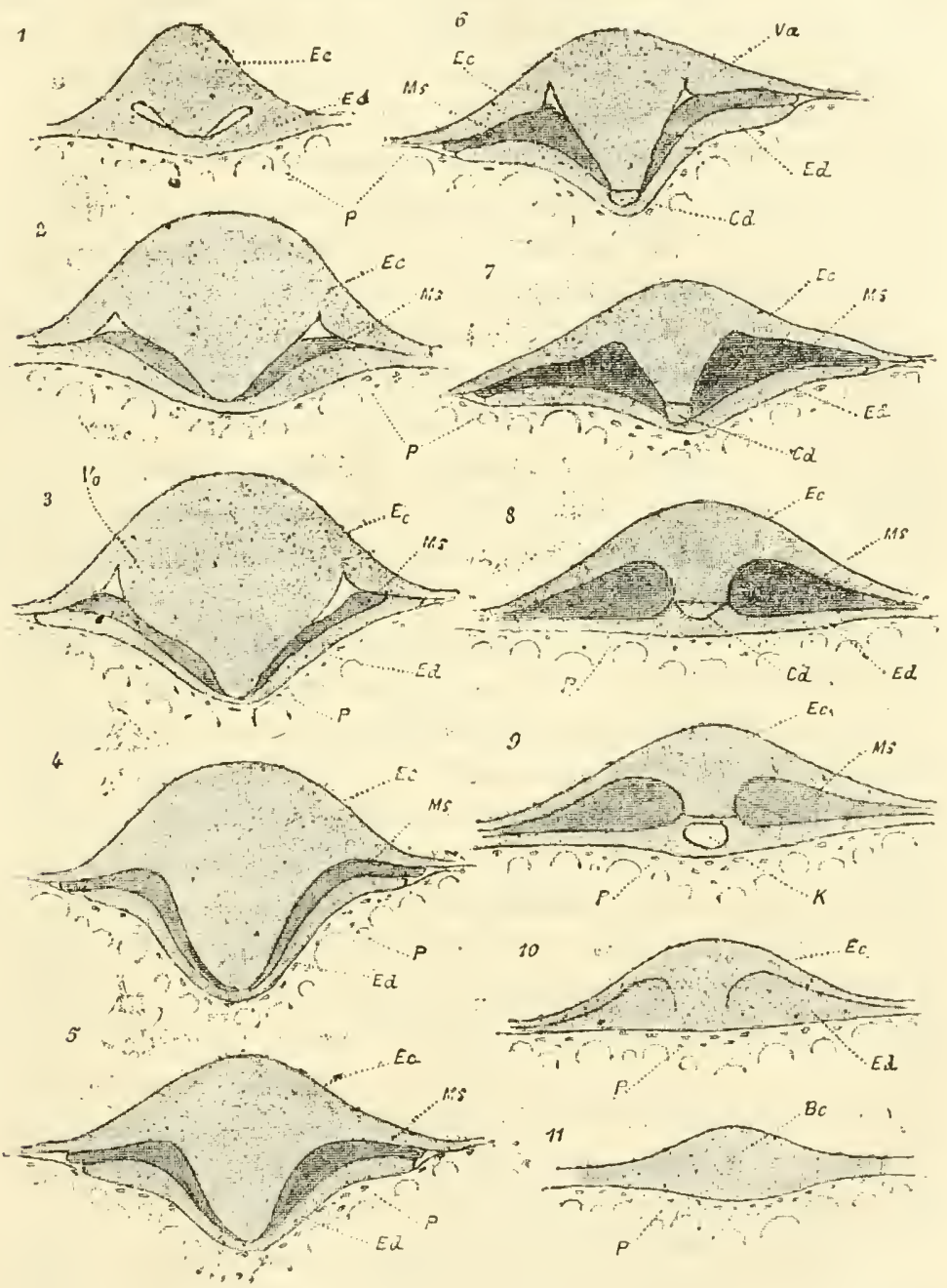

Fig. 1 a 11. - Coupes transwersales diun embryon de Truite du slate F. Ec, retoderuı"; $A s$, mésoderne; Ed, endoderme; $C d$, corde dorsale; $K$, vesieule de Kupfter; Vo, vesicule optique; $V a$, vésicule anditive; $b c$, bourgeon eardal; $P$, parablaste. Le mesoderme est plus fortement teinté que les deus antres feuillets. Ies conpes sont disposées en série, suivant lordre mumérifue, de la téte di la partie postérieuro de l'embryon.

moins en moins nette. lmmédiatement en arant du bourgeon caudal, la vésicule de liupffer apparait sur la ligne médiane, 
occupant la place de la corde dorsale. Cette vésieule se présente sous la forme d'une cavité à peu près elliptique à grand axe parallèle à la surface du vitellıs et mesurant environ $0^{\mathrm{mm}}, 09$. Sa partie inférieure est plus convexe que sa partie supérieure; ses parois sont constituées par une rangée de cellules cylindriques régulièrement disposées; sa paroi inféricure repose sur le parablaste et se continue latéralement avec l'endoderme. De chaque côté de l'axe nerreux et de la vésicule se trouvent les masses mésodermiques (fig. 9).

En arrière de la vésicule de Kupffer on aperçoit encore, dans la partic supérieure de la coupe, de chaque còté de la ligne médiane, la ligne de démarcation de l'ectoderme, mais elle cesse d'être visible sur la ligne médiane. Enfin, plus en arrière, on retrouve la disposition en cercles concentriques des cellules du bourgeon caudal, et il n'y a plus de différenciation des feuillets (fig. 10 et 11).

L'examen des coupes transversales confirme done ce que nous avaient appris les coupes longitudinales, à savoir que la vésicule de kiupffer apparait immédiatement en avant du bourgeon caudal, au point où commence à se différencier le mésoderme. De par sal situation, eette vésicule peut ître considéréc comme ayant une origine endodermique; elle repose, en effet, immédiatement sur le parablaste, et son plancher est enclavé dans l'endoderme. Son mode de développement prouve également sa nature entodermique. Le stade E nous a montré la place de la future vésicule occupée par un anas de cellules endodermiques plus grosses que les autres et régulièrement disposées. Sur des coupes d'embryons intermédiaires à E et F, on voit ces cellules se multiplier et une invagination se produire dans l'embryon pour former la vésieule.

La vésicule dont j’ai signalé l'existence chez la Truite diffère de celle que hupffer a décrite eliez d'autres Poissons par sa situation intra-embryonnaire. Celle de l'Epinoche, de la Perche, du Hareng, ete., occupe la même situation que celle de la Truite; elle est située dans le voisinage de l'extrémité caudale et à la partie postérieure de la corde dorsale, comme jai pu le constater moi-même chez la Perche, l'Epinoche et le Lepadogaster. Mais tandis que, cliez ces animaux, la vésicule fait saillie en dehors de l'embryon, dans l’intérieur du vitellus, celle 
de la Truite, an contraire, est située dans l’intérieur de l'embryon. Cette différence est sans importance, car ì un stade plus avancé lit vésicule peut faire saillie hors de l'embrỵon et devenir alors tout ì finit semblable à celle des antres loissons.

Strne G. - Les compes longitudinales de ce stade sont à peu près identiques i celles du stade F. Les somites ont augmenté de nombre: on en compte de 12 i 18. La vésicule de liupfer est bien développée et, à la partic antérieure de l’embryon, au-dessous de l'extrémité de la corde dorsale, l'endoderme commence ì so réfléchir en arrière et en-dessous pour constituer l'intestin antérieur.

L'exanen des coupes transwersiles montre que des changements importants se sont produits dans la région céphalique de l'embryon. De chaque coté de l'ise nerveux, la vésicule optique qui, an stade précédent, était ì peine ébanchée, est maintenant bien détachée, et présente la forme d'une masse ovalaire reliće atu cerveau par une partic légèrement retrécic. Les vesicules optiques arrivent au contact de la couche des cellules retodermiques. Entre ces vésicules et l'axe nerveux se trouve du tissu mésodermique enbryonnaire, rest-il-dire dont toutes les cellules sont rneore arrondies et en contact lis unes avec les autres. Le mème tissu entoure la partie inféıieure des vésicules et la carène de lave nerveus, et arrive an contact du parablaste; l'endoderme n'existe pas en ce point: tout l'endoderme primaire siest transformé en mésoderme (Planches, fig. 9!) .

Ene coupe passant en arrièro des vésicules optiques montre l'axe nerveux oceupant toute la partie médiane de la téte: de chaque côté. le mésodermo forme une ma:s a pleine qui s'itend jusqu’à l'ectoderme et an parrablaste. Une petite cavité esiste entre le mésoderme et l'ectoderme au I oint où l'embryon repose sur le vitellus.

En avant de la vésicule auditive, l'endoderme apparait. Liaxe nerveux oceupe eneore li plus grande partic de la coupe el est en contact inférieurement avec l'endoderme. Cielui-ci se relère Je chaque còté de la ligne médiane et, an-dessons de la vésicule auditive, il se produit un pli, ou plutiot une invagination, premier indice do la formation de l’intestin anterieur. En ce point. 
les cellules endodermiques sont très allongées, tandis que sur la ligne médiane elles sont aplaties et ne forment qu'une seule couche. C. K. Hoffmann (89) admet que l'endoderme n'est partout constitué que par une seule couche de cellules : suivant OEllacher (123), au contraire, il y aurait plusieurs couches de cellules; l'endoderme pénétrerait dans le mésoderme comme un bourgeon plein qui, plus tard, se creuserait d'une carité. L'cxistence de l'invagination n'est pas douteuse, mais d'un autre còté, j’ai constaté sur plusieurs préparations et à l'aide des meilleurs objectifs que. dans la partie épaissie de l'endoderme, à l'endroit où se fait l'invagination, les cellules, généralement allongées et disposées perpendiculairement au pli d’invagination, peuvent présenter aussi une forme arrondic et être alors au nombre de deux ou trois dans l'épaisseur du feuillet. Le repli endodermique arrive, sur certaines coupes, au contact mème de la partie inférieure de la vésicule auditive. sur d'autres coupes il en est séparé par quclques cellules mésodermiques (Planches, fig. 100).

Chaque plaque mésodermique est divisée en deux parties par le repli endodermique; l'une, interne, est comprise entre l'ectoderme, l'axe nerveux, la corde dorsale et l'endoderme; ses cellules commencent à prendre un aspect particulier, à sallonger dans le sens de la hauteur de l'embryon et se disposer sérialement; l'autre, externe, est comprise entre la partie aminrie de l'ectoderme qui fait suite à la vésieule autidive, la partie externe du repli endodermique et le parablaste. Cette partic externe du mésoderme est creusér d'une cavité, la carité du cœlome. le toit de la cavité est folmé par une couche de cellules plus épaisse que celle qui constitue le plancher.

Les coupes suivantge présentent á peu près le mème aspect que les précédentes; l'invagination de l'endoderme y est moins marquée, et cesse un peu après le tiers antéricur de l'embrỵon.

Dans la partic postéricure du corps do l'embryon, on retroupe la mème disposition qu au stude précédent, les masses protoxertébrales sont mieux accusécs et plus nettement diffireneięes des plaques mésodermiques laterales.

aidest à ce stade, ou plutòt entre fo starle $G$ et le stade II. que se forme la cavité centrale du système nerveus. Cefte ques tioft très discutéc a donné lieu à plusieurs interprétations. Nous 
y reviendrons en décrivant le développement du systęme nerveux.

Stade H. - Une coupe médiane longitudinale d'un embryon de $3^{m m}, 2$ et possédint 22 somites, montre l'axe nerveux et, audessous de lui, la corde dorsale dont les cellules allongées tranchent nettement sur le reste de la coupe. L'extrémité antérieure de la corde dorsale est à $0^{\mathrm{mm}}, 7$ de l'extrémité céphalique; au-dessous de liı corde et un peu en arrière de sa terminaison, l’endoderme présente une fissure qui est l'origine de l’intestin antérieur. En arrière do l'extrémité postérieure de la corde dorsale se trouve la vésicule de hupffer qui, comme au stade précédent, s’est allongée antéricurement et conımunique avec l'intestin postéricur (Pl. fig. 86).

Sur les coupes longitudinales latérales, on voit, ì la partic antérienre de la région céphalique, la vésicule optique dont la partie externe s’est invaginée dans la partie interne et qui en est séparée par une cavité linéaire. Plụs cn alrière, se trouve la résicule auditive arrondie et détachée du feuillet externe. Au-dessous de la vésicule auditive, le feuillet interne reployé sur lui-même est situé au milieu du mésoderme et présente une courbure à convexilé supérieure et postérieure; il se contimue postérieurement avec la partir de l'endoderme qui est en contact avec le parablaste, el qui représente l'intestin moyen. La partie de l'endoderne comprise aiusi dans l'épaisseur dı mésoderme est la région dins laruclle se formeront plus tard les fentes branchiales. Dins cette même région, le mésoderme, situé au-dessous de l’intestin antérieur, présente une grande cavité, portion péricardique du colomr. An-dessous de la lame inférieure, qui constitue le plancher de cette cavité, existe une antre cavité plus petite, limitée par une couche de cellules aplaties et reposant directement sur le parablaste, c'est l'ébauche de l'une des moitiés du coutr (Pinches, fig. 87).

En arrière te la vésicule anditive, il y a une masse mésodermique indivise qui précède la série de protovertèbres; cellesci sont pressées les unes contre les autres, et ont la forme de rectangles allongés verticalcment et arrondis à leurs extrémités supérieure et inférieure. Les cellules qui constituent une protovertèbre sont disposées perpendiculairement à la ligne de 
contour; dans l'intérieure de la protovertèbre existe une cavité virtuelle ('lanches, fig. 111).

Les coupes transversales du stade $\mathrm{H}$ méritent d'être étudiées avec soin parce qu'elles montrent la différenciation des principaux organes.

La tête tend déjà à se soulever au-dessus du vitellus et à s'en détacher. L'ectoderme qui forme la couche épidermique de l'embryon ne se continue plus directement avec la couche externe du sac vitellin : mais, au point où l'embryon repose sur le vitellus, l'ectoderme s'infléchit au-dessus de lui et forme une lame qui s'avance vers l'endoderme primaire différeneié en mésoderme, puis se réfléchit à la surface du vitellus pour se continuer avec la paroi du sac vitcllin. Cette disposition est nettement visible au-niveau des vésieules optiques; ces vésicules ne sont plus en rapport avec l'axe nerveux que par leur pédicule, ou nerf optique, qui se détache de la partie inféricure de la vésicule cérébrale antérieure. La cavité de la vésicule optique se continue par le pédicule avec celle de l'axe nerveux: des deux couches de la résieule, l'interne est plus minee que l'externe; celle-ci présente de nombreuse cellules en eỹtodiérèse, surtout à sa face interne : cette face est, en effet, le siège d'une croissance rapide qui entraine la courbure à convexité interne de l'ensemble de la vésicule. Dans la eoncavité externe de la vésicule est logé un épaissement de l'ectoderme, le futur cristallin (fig. 12 et 13) (Planches, fig. 103).

En arrière des yeux, le cerveau offre une section elliptique, dont la partie supérieure est plus large que la base. Le mésoderme présente à ce stade, dans la région céphalique, de chaque côté de l'axe nerveux, un aspect particulier. Les cellules ne sont plus arrondies ou polygonales, ni pressées les unes contre les autres. Elles sont allongées avee des prolongements filiformes s'anastomosant entre eux; entre elles, existent des lacunes irrígulières. Cette portion du mésoderme revêt les caractêres du tissu conjonetif, dans lequel il n'y a pas encore de substance intercellulaire (fig. 14).

Entre les vésicules optiques et les vésicules auditives, on trouve l'invagination endodermique qui a donné naissance à l'intestin antérieur. La section transversale de cet intestin a la la forme d'un fer à cheval, dont la concavité embrasse la partie 
inféricure de l'axe nerveux et dont les extrémités arrivent au
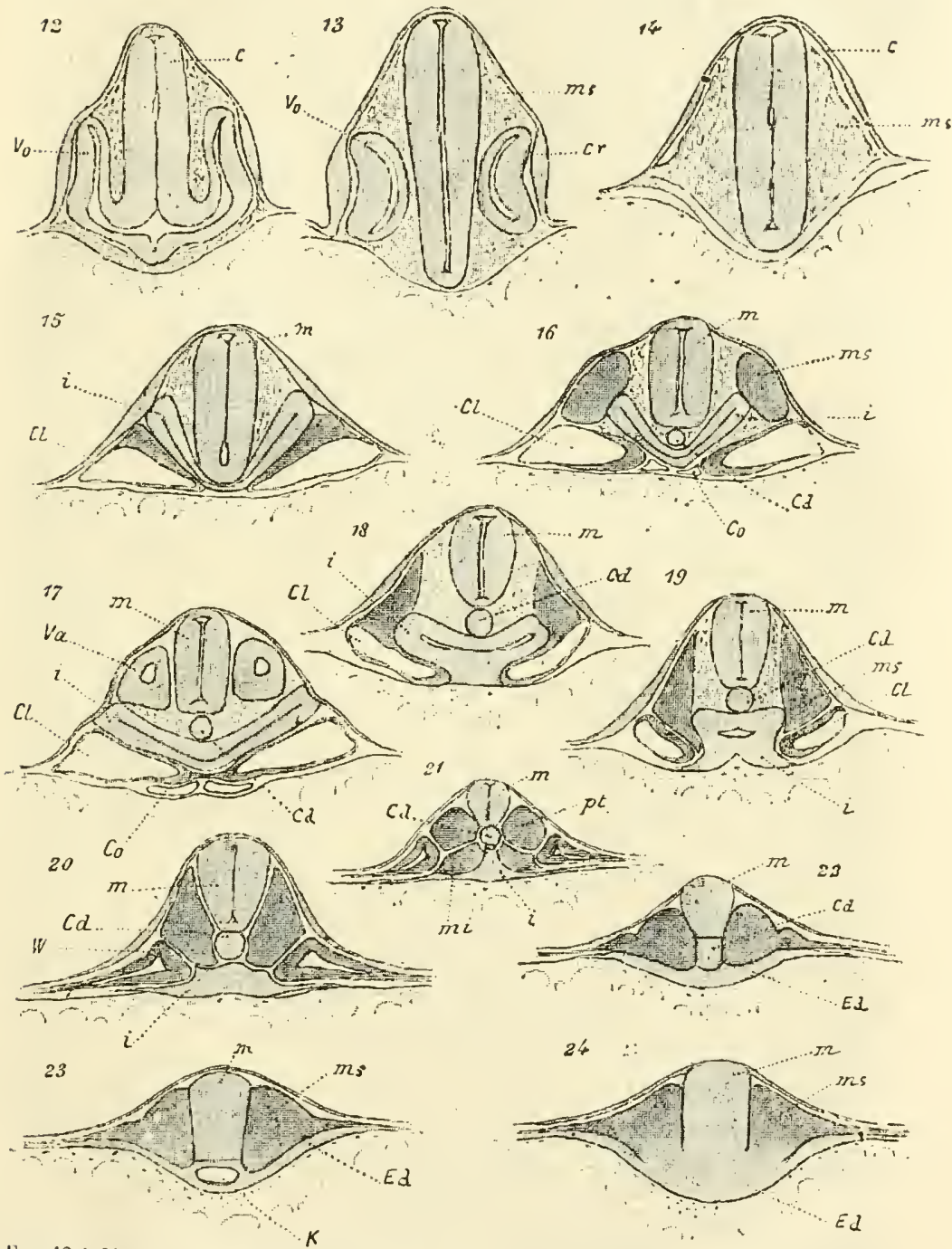

Fig. 12 a $2 \%$ - Coupes transversales d'un embryon de Truiet du stade II. $c$, cerveau: m, moẻlle épinière; $M s$, mésodermo; $E d$. endoderme; $C d$, corde dorsale; $K$, vésicule $p t$, protovertebres anditive. Lc mésoder, intestin; Vo, vésicule optique; $\mathrm{Cr}$, eristallin; $V a$, vésicule' coupes sont disposées en série, suivant leinte que les deux autres feuillets. Les posterieure de l'embryon.

contact de l'ectoderme, ou en sont séparées par une masse mésodermique. Cette masse mésodermique a conservé le carac- 
tẻre primitif et est constituée par des cellules pressées les unes contre les autres; elle diffère du tissu conjonctif, qui entoure l'axe nerveux, et elle donnera naissance à des muscles. Le mésoderme situé au-dessous de l'cndoderme, de chaque côté de la ligne médiane, cst également formé de cellules embryonnaires; dans son épaisseur, se trouve la cavité du colome. de forme triangulaire, le flus gand côté du triangle reposant sur l'intestin (fig. 10 et 16 ).

C'est au nireau des vésicules auditires que l'intestin antérieur est le plus développé. Sil section oceupe toute la largueur de la coupe; clle s'étend d'une manière presque rectiligne d’un cólé de l'embryon à lautre, au-dessous dr la corde dorsale et de deux amas de tissu conjonetif qui la séparent de l'axe nerveux. Chaque extrémité de la section est en contact, par sa partie supéricure, avec la vésicule auditive, et en rapport intime avec l'ectoderme. En ce point, il n'existe plus trace du pli d’inragination de l'endoderme; la cavité rirtuclle de l'intestin arrive jusqu'an contact des cellules ectodermiques; celles-ci sont refoulées en bas et en haut par les cellules endodermiques, qui ne sont plus recouvertes que par la couche enveloppante. Cette disposition est en rapport avec la formation d’une lente branchiale. Par suite de la résorption de la couche enveloppante, la cavité de l’intestin communiquera plus tard directement arec l'extérieur, la paroi supérieure el la paroi inférieure de l’intestin se continuant avee l'ectoderme (fig. 17).

Au stade H apparaissent également les premiers rudiments du coeur. Sur la ligne médiane de l'embryon, la partie inférieure de lintestin présente un épaississement qui s’arance comme un coin vers le vitellus et sipare les deux carités cœlomatiques, creusées dans l'épaisscur des lames mésodermiques latérales. Il résulte de cette disposition qüil existe entre l’intestin, les deux lames mésodermiques et le parablaste, un espace libre, de forme plus ou moins irrégulière, suirant que l'intestin est plus ou moins rapproché du parablaste. De chaque angle interne des deux lames mésodermiques latérales, se détachent des cellules qui s’agencent en une conche d'éléments aplatis qui sont l'origine de l'endolhélium cardiaque. Ces cellules se disposent, en effet, de manière à constituer deux tubes parallèles, sitnés de charque coté de la ligne médiane de l'em- 
bryon, et reposant directement sur le parablaste. Bientòt de nouvelles eellules se détachent des lames mésodermiques, s'avancent vers li ligne médiane et finissent pä former une couche cellulaire qui réunit les deux lames mésodermiques, en séparant lintestin des deux moitiés cardiaques. Dans lintérieur de chaque tube endothélial eirdiaque on roit quelques cellules arronlies. libres, qui sont les premiers globules sanguins; jaurai is discuter plus loin, lorsque je moccuperai des dérivés du mésoderme, les diverses opinions émises par les auteurs relativement à l'origine des globules sanguins et du cœur chez les Poissons (Planches, fig. 104 et 114).

En arrière de la région cardiaque, les coupes transversales montrent, de chaque côté de l'embryon, un épaississcment de l'ectoderme en rapport avec une dépression qui existe dans les masses mésodermiques. L'ectoderme, en ce point, est formé de deux rangées de cellules; cet épaississement est la première ébauche de la ligne latérale. Aux stades ultérieurs, la masse cellulaire, largement étalée au stade $\mathrm{H}$, se concentre vers le milieu de la hauteur de l'embryon, et se creuse d'une gouttière qui finit par se transformer en canal latéral (fig. 19).

Sur les mêmes coupes, l'endoderme est concentré sur la ligne médiane. Sa section transversale a la forme d'un trapèze dont le plus grand còté, légèrement curviligne, à convexité supéricure, est situé au-dessous de la corde dorsale, tandis que le petil côté repose sur le parablaste. Aux angles supérieurs du trapèze. les cellules, allongées et dirigées vers la cavité de lintestin, indiquent les plis d'invagination, qui se son tproduits de la même manière que dans la partie antérieure de l'embryon (Planches, fig. 10:̈).

Vers le milieu de lembryon, le mode de formation de lintestin est différent. Il ne se forme plus qu'un seul pli d'invagination de l'endoderme, du parablaste vers la corde dorsales sur la ligne médiane. Au niveiu de ce pli, les cellules sont allongées dans le sens de l'épaisseur du feuillet, ou disposées en deux couches. En dehors du pli, l'endoderme est étalé à la surface du parablaste, mais il ne dépasse pás le milieu de chaque lame nésodermique latérale. A la partic supérieure lu pli d’invagination, il se détache de l'endoderne un petit grompe de cellules qui se disposent en une petite masse arrondie, immédiatement 
en contact avec la corde dorsale. Ce groupe cellulaire, qui se retrouve sur plusieurs coupes consecutives, est la lige sousnotocordale (fig. 21) (Planches, fig. 106).

On peut distinguer dans le mésoderne de cette région trois parties bien différenciées. De chaque còté de l'axe nerveux et de la corde dorsale se trouvent les masses protovertébrales dont les cellules périphériques sont régulièrement disposées. Au-dessous de ces masses, les lames mésodermiques latérales, divisées, comme dans la partie antérienre de l'embryon, en somatopleure et splanchnopleure, ne présentent plus dans leur épaisseur qu'une cavité virtuelle du colome. La portion interne de chaque lame mésodermique tend à se séparer du reste par suite d'un étranglement qui se produit dans le somatoplemre. Cette portion interne est relevéc en haut vers la protovertèbre, et renferme dans son intérieur un prolongement le la cavité du colome. La partie ainsi replice de la lame mésodermique est la première ébauche du canal de Wolff. Entre la partie inférieure de lis protorertèbre, le canal de Wolff et l'intestin, se trouve une masse plus ou moins arrondie de cellules nésodermiques, aux dépens de laquelle se développeront plus tard des vaisseaux et probablement aussi du tissu conjonctif (fig. 20).

Dans la région postérieure de l'embryon les coupes reprennent l'aspect qu'elles avaient aux stades précédents. L'axe nerveux n'a pas encore de cavité centrale: l'endoderme est largement étalé sur le parablaste: il est seulement épaissi en son milieu, et offre une petite cavité sur la ligne médiane. La corde dorsale est plus haute et plus large; ses cellules sont moins différenciées (fig. 22).

En avant de la vésicule de Kupffer, le mésoderme forme, de chaque còté de l'axe nerveux et de la corde dorsale, une masse non différenciée de cellules embryonnaires, dans laquelle on ne distingue plus, ni masse protovertébrale, ni lame latérale avec cavité de coelome

Au niveau de la vésicule de hupffer. la corde dorsale a disparu. Liendoderme, très épais sur la ligne médiane, arrive au contact de l'extrémité inférieure de l'axe nerveux, dont il n’est séparé que par une ligne de démarcation à peine marquée. La vésicule de Kupffer, dont la section transversale est elliptique, a sa paroi inférieure, reposant sur le parablaste, constituée par 
une rangée de cellules cylindriques. Sa paroi supérieure est également formée d'une couche de cellules eylindriques, mais au-dessus dielle, les cellules endodermiques sont allongées parallèlement au grand axe de la vésicule. comme le sont les cellules ectodermiques dans l'axe nerreux (fig. 23) (Planches, fig. 107).

En arrière de la vésicule, l'ectoderme est bien différencié des parties voisines dans sa portion supérieure, mais. inférieurement. il se continue en une masse de cellules indifférentes dans laquelle il est impossible de distinguer les feuillets; l'endoderme n'est séparé du mésoderme que sur les còtés de l'embryon. On doit done considérer comme appartenant à l'endoderme primaire cette masse cellulaire qui est en continuité directe avec l'ectoderme, en arrière de la vésicule Kupffer (fig. 24).

Enfin, au niveau du bourgeon caudal, on ne trouve plus qu'une masse de cellules embryonnaires présentant une disposition concentrique, identique à celle qui existe dans le cordon axial dès les premiers stades du développement.

J'ai déjà dit, en décrivant la conformation extérieure de l'embryon aux différents stades, que c'est au stade $H$ qu’a lieu la fermeture du blastoderme en arrière de l'embryon. Les coupes pratiquées à travers le blastopore vitellin, ayant la forme d'une petite ellipse très allongée; montrent de quelle manière se fait la réunion des deux moitiés du bourrelet blastodermique.

Les coupes passant par le milieu blastopore vitellin montrent en leur milieu un espace vide. limité inférieurement par le parablaste, de chaque còté par le bourrelet blastodernique et en haut par une couche de cellules très aplaties qui est la lame enveloppante. Celle-ci passe comme un pont d'un bourrelet blastodermique à l'autre. Je n'ai pu déterminer exactement de quelle manière et à quel moment se fait la réunion de la lame enveloplante d'un côté à celle du côté opposé. Il est probable que la lame enveloppante qui, comme on le sait, ne prend pas part à la réflexion de l'ectoderne au niveau du bourrelet blastodermique, s’étend ì la surface du parablaste, lorsque le blastopore est sur le point de se fermer, et que lorsquelle s’est réunie à celle du còté opposé, il se fait au-dessous d'elle une accumumulation de liquide qui la soulève et lui donne la disposition qu'elle présente sur les compes. A mesure que les dem 
bourrelets blastoderniques se ripprochent l'un de l'autre, il se produit une résorption de cellules dans la lame enveloppante, ce qui fait qu'elle reste tendue au-dessus du blistopore.

Chaque bourrelet blastodermique conserve la structure quii] avait dès le début du développement embryonmaire; il ne renferme que deux feuillets, l'eetoderme et l'endoderme primaire confondus au borl libre du bourrelet, là où s'est produit la réflexion de l'eetoderme. Au voisinage du point de fusion, l'ectoderme est plus épais que l'endoderme primaire, et est formé de cinq à six couches de cellules, puis il se continue en une eouche unicellulaire à la surface du vitellus. L'endoderme primaire, au contraire, d'abord constitué par trois ou quatre rangées de cellules, offre un épaississement au point où l'ectorlerme commence ì s'amincir, puis il s'ètend à une petite distance en une couche de cellules disposées irrégulièrement (Planches, fig. 97 et 98 ).

Si l’on examine la série des coupes successives pratiquées à travers cette région, on peut suivre tous les degrés de la fusion des deux lèvres du blastopore. On voit les deux bourrelets blastodermiques se rapprocher l'un de l'autre, arriver au contact et se fusionner. Le milieu de la coupe est occupé par une masse de cellules indifférentes, semblable à celle qui existe en arrière de la résicule de Kupffer; sur les côtés se trouvent l'ectoderme et l'endoderme primaire. La ressemblance de ces coupes arec celles de la région postérieure de l'embryon immédiatement en avant du bourgeon caudal pourrait être invoquée à l'appui de la théorie de la concreseenee de His, qui fait provenir l'embryon de la soudure des deux moitiés d'une anse du bourrelet blastodermique. Je discuterai cette théorie dans un chapitre spécial consacré à l'accroissement de l'embryon.

Lorsque le blastopore vitellin est complètement fermé, la masse cellulaire, résultant de la fusion des deux lèvres du blastopore, s’ajoute à la partic postérieure de l'embryon et se fusionne avec le bourgeon caudal. Cette masse se différencic plus tard, comme le bourgeon caudal, en tissu nerveux et en protovertèhres, mais, pendant longtemps, l'axe nerveux el l'endoderne restent unis sur la ligne médiane, ainsi que cela s'observe en arrière de la vésicule de K!nıffer an! stade $\mathrm{H}$. 


\section{DEUXIEYE PARTIE}

Ians la première fartic de ce travilil, jai décrit successivement lasprect des conpes longitudinales et transversales des embryons aur différents starles, depuis la formation de l'écusson embryonnaire, jusqu'à la fermeture du blastoderne. J'ai indiqué, pour chaque stade, l'apparition des noureaux organes et la transfornaiuon des organes dejà existants. Cette méthode chronologique, qui présente de grands avantages pour suivre les progrès du développement de l'embryon, ne renseigne que d'une manière imparfaite sur l'évolution de chaque système, de chaque organe en particulier. Les divisions purement artificielles en stades, qu'on est obligé d'établir pour la commodité de l'exposition, font qu'on néglige d' décrire les modifications dont les organes sont le siège dians lintervalle de deux stades consécutifs. Il est donc de toute nécessité, après avoir donné le mode de développement d" l'embryon, de considérer les transformations de chaque organe, c'est-i-dire de faire l'organogénie de l'embryon.

de pisserai rapidement en revue l'évolution des trois feuillets en énumérant les organes auxquels chacun d'eux donne naissance et en completant lhistoire du développenent de ces organes. de discuterai également à propos de chacun d'eux les opinions des différents auteurs, n’ayant exposé dans les chapitres précédents que le résultat de mes propres recherches.

\section{VII. - LiGGOHEME ET SES DÉMTÉS}

De même que chez les autres Vertébrés, l'ectoderme des poissons osseux donne naissance à l'épiderme, au système nerveux central et aux organes des sens spéciaux.

L'ectoderme est formé, dès le début, de deux couclies de cellules; la couche supérienre (lame enceloppante, lame recourrante, lameépidermique, lame cornée, lame limitante) est constituce parr une seule rangée de cellnles qui se différencient de très bonne heure, amsi que nous l'atrons dejà vu dans létude de lis segmentation. Cette enuche ure prend pals part à las 
réflexion de l'ectoderme pour constituer l'endoderme primaire, ni à la formation de laxe nerveux; elle reste toujours appliquẻe sur la couche profonde de l'ectoderme. On peut la suivre pendant tout le développement de l'embryon; elle s'amincit de plus en plus et forme à la surface de la peau du jeune Poisson une membrane nucléée exeessivement mince.

La couche profonde de l'ectoderme est au contraire constituée, dès son apparition, par plusieurs rangées de cellules et peut être désignée, avee la plupart des auteurs, sous le nom de lame nerveuse, ou, arec Gœtte (60), sous le nom de couche fondamentale de l'ectoderme. C'est, en effet, à ses dépens que se développent le système nerveux central et les organes des sens spéciaux.

L'existence de la lame enveloppante n'est pas propre aux Poissons osselix. Reichert (142) l'a décrite le premier chez la Grenouille; Salensky (163) l'a vue chez le Sterlet et Balfour (10) chez le Lépidostée. Chez ces animaux elle est également constitıée de très bonne lıeure par une couche unique de cellules, qui s'aplatissent pour former une lamelle très minee. Mais, tandis que, chez les Amphibiens anoures et le Sterlet, cette couche entre dans la constitution du système nerveux central, elle demeure étrangère à cette furmation chez le Lépidostée et les Téléostéens.

La lame enveloppante manque, ou du moins n'a pas encore été signalée chez les Plagiostomes, les Cyclostomes, les Amphibiens urodèles, les Reptiles et les Oiseaux. Chez les Mammifères on la retrouve avec une existence transitoire. La conche que Rauber (136) a décrite chez le Lapin et qu'on désigne sous le nom de couche de Rauber peut, en effet, être assimilée à la lame enveloppante des Vertébrés inférieurs. Cette couche, qui n’est que la partie de la résicule blastodermique recouvrant le reste vitellin de Bischoff, ne constitue pas l'ectoderme, comme on le croyait autrefois, avant les recherches de Rauber, de Lieberkïhn (115), de Hensen (77), de liœlliker $(\mathbf{9 7}$ bis) et de Heape (66). Liectoderme proprement dit et l'endoderme proviennent de la masse interne des cellules blastodermiques. La couche de Rauber, qui recourre l'ectoderme, disparait de bonne heure chez le Lapin, soit par résorption, soit plus probablement par intégration dans l'ectoderme. Mais, chez 
d'autres Mammilères, elle prend un développement plus ou moins considérable, permanent ou temporaire, of ronstitue d'après Kupffor (105), Sclenka $(\mathbf{1 7 5}, \mathbf{1 7 6})$ et Heape, le suspenseur de l'muf de rertains Rongeurs, Souris, Rat, Campagnol, Cochon d'Inde.

Le reste vitellin de Bischoff me semble entièrement comparable au germe segmenté les Téléostéens après la formation de la lame enveloppante. C'est, en effet, aux dépens du germe et du reste vitellin que se constiturnt les feuillets primaires, tandis que la couche enveloppante et la couche de Risiber ne prennent pas part it la formation de ces feuillets.

Balfour ( $\mathbf{9}$ divise les Vertébris en deux groupes an point le vue de la constitution de leur ectorlerme: un groupe nombreux dont l'ectoderue n'est formé que par une seule couche de celJules ; un petit groupe dont l'ectoderme comprend deux couches. Il pense que l'état monoblastique est la eondition primordiale de l'ectoderme, tandis que l'état diblastique répond ì une différenciation produite à une période très précoce du développement.

La couche profonde de l'ectoderme, qui, à lat fin de la segmentation, forme le toit de la cavité germinative, n’est eonstituée, en tant que feuillet, qu'au moment où prend naissance l'endoderme primaire par réflexion du blastoderme. Cette conche, composée de plusieurs rangées de cellules arrondies, prend un grand développenent an niveau du rudiment embryonnaire, tandis quelle samineit et se réduit à une seule rangée de cellules sur le reste du blastoderme, excepté cependant dans le bourrelet blastodermique. Elle donne naissance a l'épiderme de l’embryon et à l'enveloppe du sac vitellin.

Lectoderme proprement dit prolifère sur la ligne axiale de l'embryon pour produire l'élauch du système nerveux central: sur les cotés, il tend, au contraire, à s'amincir au fur et à mesure que l'embryon se développe, pour ne plus être formé, au stade $\mathbf{H}$, que par une seule rangée de cellules épidermiques, recouvertes par la lame enveloppante. Au niveau de l'embryon, les cellules épidermiques sont eylindriques, sauf dans la partie située au-dessus de l’axe nerveux; en ce point, elles sont aplaties jusqu'au stade G, c'est-à-dire tant que l'axe nerveux conserve des rapports avec la couche profonde de l'ectoderme. 
Lorsqu'au contraire l'axe nerreux s'est complètement séparé de cette couche, les cellules épidermiques, qui le recouvrent, prennent une forme eylindrique.

Une semblable disposition s'observe ehez le Poulet, même quelque temps après que In système nerveux s'est bien différencié de l'épiderme. Dans les parties extra-embryonnaires les cellules épidermiques sont aplaties, comme celles de la couche enveloppante, mais moins que ces dernières.

Au stade Il, de chaque còté du corps de l'embryon, au niveau des masses protovertébrales, lépiderme présente un renflement à saillie interne. Les cellules, en ce point, sont plus allongées dans le sens verlical que dans le reste de la couchr. Ce renflement est, ainsi que nous l’avons déjà vu, le rudiment du canal de la ligne latérale, qui n'existe d'abord que dans la région antéricure de l'embryon (l'lanclies. fig. 10\%, $t t$ ). Balfour (9) avait déjà constaté cette bande linéaire d'épiderme modifié chez le Saumon, mais seulement au moment de l'éclosion. C. K. Hoffmann (90) l’a vue également ì un stade moins avancé du développement. Il décrit et figure sa première apparition sous forme d'une prolifération locale des cellules épidermiques. J. Beard (14) a lien suivi le développement de la ligne latérale et la fait provenir d'un cordon eellulaire qui se détache de la couchr profonde de l'ectoderme, dabord dans la région cervicale pour s’étendre ensuite en arrière daus toute la longueur du corps: mais cet auteur ne parait pas avoir vu le début de la formation de cet organe. Un mode semblable de développement de la ligne latérale a été signalé par Semper (17\%) et Balfour (8) chez les Pagiostones et par Gœlte (59) chez le Bombinator.

Systeme nerecur. - Les promiers observateurs qui so sont occupés du développement du système nerveux des Poissong osseux, Rathke, Baer, Vogt. Lereboullet. Stricker, pensaient que laxe cérébro-spinal so formail comme colui des Amphibions of des Amniotes par un épaississement du feuillet externe of liapharition dime gouttière qui se fermait sur la face dor= sale de lembryon de manière à conslituer mu lube.

limpifer (104, le preminer, reconmut que le systeme nerrens eentril des Tréosténs est au début une formation solish de 
l'ectoderme. Il vit qüil se produit bien à un moment donné un sillon superficiel, comme chez les autres Vertébrés, mais que ce sillon n'i qu'une existence temporaire et superficiellr. que ses bords ne se rejoignent pas, et que lade nerveux est primitivement un cordon plein, ayant inférieurement la forme d'une carène qui s'enfonce comme un coin dans le vitellus. Gette (60), Schapringer (165), Weil 195), OEllacher (123), Hoffmann (89) confirmèrent la découverte de Kupffir. Gotto montra que l’apparition du sillon superficiel ef celle de la carène nerveuse sont simultimées.

OEllacher admet que lis conche profonde de l'ectoderme. Ie fenillet sensoricl, ne preml pas part à la formation de l'épiderme et donne naissanee an systimr nerveux eentra\}. Celui-ci apparait sous forme d'un cordon axial (Axenstrang) dans lequel les cellules ont une disposition an couches concentriques et qui s'étend dans toute la longueur de l'embryon. Dans ce cordon, les couches des cellules du feuillet sensorial se continuent avec les couches des cellules non encore différenciées dı feuillet profond; il ny a, à ce niveau, aucune ligne de démarcation entre l'eetoderme et la masse qui donnera le feuillet moyen et l'endoderme. Le cordon axial se termine cu arrière dans le bourgeon caudal, dans lequel il n'y a aucune diflérenciation de feuillets. A la surface de l'écusson embryonnaire, en avant du bourgeon caurlal, il existe un sillon longitudinal médian, le sillon médullaire, qui suétend au-rlessus du cordon axial. Celui-ci fait saillie ì lit face inférieure de l'embryon et constitue une carène qui s'enfoner dims le viteklus. Le sillon superficiel seetend en avint et devient plus profond à mesure que le cordon axial prend lui-mème un plus grand développement dans to sens postéro-antérieur at s’épaissit verticalement. Dans le cordon axial, OEllacler distingue trois parties : une partie céphalique, une partie caudale et entre les deux une prirtic qui correspond au trone de l'embryon. Plus tard, le cordon axial se divise horizontalenent en deux jortions, l'une smpérieure en rajport avee lo fenillut sensorin et qui eonstitue l'axe nerveux, l'autre inlérieure, formée par un groupe arrondi de cellules at qui représente la corde dorsale.

Pour Romiti (148) et Calbella (31), le développement du systeme nerveus dos Poisons asseux se ferait palr un pro- 
cessus identique à celui quon observe chez les autres Vertébrés, cist-ii-dire par une invagination de la couche superfirielle de l'ectodermo. Calberla a fait porter ses recherches sur le Syngnathe et le Simmon, ainsi que sur la Lamproie. Chez le Syngnatloe, les cellules de lia lame enveloppante sont assez grosses et prismatiques; au fond du sillon médullaire on observe une double rangée de ees cellules, qui s'enfonee verticalement dans la carène nervuse. II se produirait, d’après cet auteur, une sorte de plissement de l'ectoderme qui emprisonnerait ainsi les eellules de la eouche superficielle. L'invagination de laxe nervemx, au lieu de se faire, comme chez les autres Vertébrés, sous forme diune gouttière, dont les bords se rapprochent pour constituer un canal, se ferait sous forme d'un simple pli; la gouttière serait comme écrasée latéralement.

Gette (60) repousse, avec juste raison, la manì̀re de roir de Calberla. Pour lui, lase nirveus est bien un cordon solide creusé ì sa partie supérieure d'un sillon superficiel représentant le sillon médullaire. Le sillon se ferme jar un plissement latéral de la couche ectodermique, mais sans emprisonner la lame enveloppante, qui passe comme un pont au-dessus du sillon.

Ilis 85) arlmet, comme Calherla, la formation diun pli d’invagination, et il donne dans sa figure 7 le schéma de la formation de laxe nervems. Les deus moities dn pli llinvagination s`appliqueraient exactementl'un contre l'autre, de sorte que la lumière du canal serait remplacée par une ligne verticale. Le canal médullaire résulterait ultérieurement de la séparation des deux moitiés du sillon.

C.-K. Hoffnamn (89) adopte pour la formation du système nerveux lopinion de Gette. Il se forme sur la ligne médiane de l'embryon une cirène ietodermique. présentant à la partic supérieure un sillon superficiel, au-dessus duquel la lame enveloppante pass comme un pont. Iloffmamn nal jamais ru se produir de pli d’invagination: les cellules au-dessous du sillon médullaire sont serrées les unus contre les autres comme dans les autres parties de l'embryon.

Ziogler $(\mathbf{2 0 0})$ a une opinion intermédiaire entre celle de Goute rt celle de Ilis; il almet une invagination de l'ectoderme sous forme de pli ferme. invagination à larpuelle la lame euvelopplante ne semble pas premlre part. 
Enfin Goronowitsch (5y), qui a publié, en 1884, un mẻmoire important sur la question, décrit la formation successive de deux sillons dorsaux dans la région médullaire. Le premier sillon coïncide avec l'apparition de la lame médullaire, constituée au début par une partie médiane mince et par deux parties latérales épaisses. Le premier sillon disparait hientót par suite de l'épaississement progressif de la région médiane. Le second sillon, qui apparait plus tard, résulte d'une invagination de la surface de la lame médullaire; il persiste plus longtemps que le premier sillon, et, lans la région céphalique, présente deux fossettes, l'une antérieure ovale, l’autre postérieure irrégulièrement rhomboïdale, et correspondant, la première au cerveau antérieur, la seconde au cerveau postéricur. La lame enveloppinte passe au-dessus du second sillon. Le plancher de ce sillon est formé par une masse de cellules arrondies qui sont nettement distinctes des autres cellules de la carène médullaire; un certain nombre de ces cellules superficielles péuètrent dans le tissu central de la carène.

Avant d'aborder la discussion des différentes nanières de voir des auteurs que je viens de citer, il me reste ì exposer les idées de kupffer (106) relatives au développement de l'embryon et en particulier du système nerveux. Les figures que Kupffer donne des preniers stades du développement de la Truite diffèrent énomément de celles de sos doranciers. Suivant cet auteur, an huitième jour de lincubation, c’est-à-dire au stade $\mathrm{C}$, le blastoderme présente en un point de sa circonférence une petite saillie proéminente, qui est le hourgeon caudal d'OEllacher; en avant de celui-ci, se trouve un épaississement blastodermique. l'écusson embryonnaire de tous les auteurs, mais qui, pour hupffer, n’appartient pas encore à l’embryon. Bientot, il se produit en avant du bourgeon caudal, sur la ligne médiane de l'écusson, une invigination sous forme d'un sillon longitudinal; puis apparait un antre sillon perpendiculaire au premier, mais dont l'existence est temporaire. Il ne reste plus que le sillon longitudinal (gouttière primitive, Primitivinne) qui sallonge en même temps que l'écusson blastodermique. Les bords de la gouttière primitive se réunissent en avant du bourgeon caudal et forment un cordon axial médian, que kupffer considère comme une ligne primi- 
tive (Achsenstreif oder Primitivstreif). Ce cordon axial ou ligne primitive est le résultat d'une invagination ectodermique. Vers le commeneement du onzième jour, la gouttière primitive a disparı et la bautlelette axiale, qui s'est élargie dans sa partie antérieure, occupe la ligne médiane de l'embryon. La disparition de la gouttière primitive coïncide avec le moment où le blastoderme a recouvert une moitié du vitellus. A ce stade, qui correspond à notre stade F. il n'y a pas encore trace d'embryon. "Es gibt also, dit hupffer, einen Zcitpunct in der Entw icklung des Forelleneies, wo äusserliel jede Spur der Einstülpung, die sich zuerst als tiefe Grube mit vorherrschend querer Dimension, dann als longitudinale Primitivrinne zeigt, verschwunden ist, ohne dass sich ein Embryo mit Kopftheil und Metameren des Rumpfes erblicken liesse. *

Pour Kupffer l'embryon se forme d'une manière indépendante de ce qu'il considère comme une ligne primitive; la tête apparaît d'abord en avant de l'extrémité antérieure de la bandelette axiale : elle consiste dans le rudiment du cerveau, des yeux et d'une paire d'arcs branchiaux; elle se continue avec la bandelette axiale, dont elle est séparée par une constriction. Les protovertèbres, qui apparaissent ensuite, se développent en dehors de la bandelette axiale; la moelle au contraire se forme dans cette bandelette et est en continuité avec le cerveau. En résumé, pour hupfer, l'embryon des Poissons osseux ne se forme pas, comme on l'a admis jusqu'à présent, directement au contact du bourrelet blatodermique : son apparition est précédée de celle dune ligne primitive résultant d'une invagination linéaire de la surface du blastoderme, et c'est à l'extrémité antérieure de cette ligne primitive que se développe l'embryon, la tête apparaissant la première.

Kupffer n'a pas encore figurë ni décrit les coupes qu'il a faites des embryons qu'il a étudiés : il serait intéressant de savoir comment il peut concilier sa théorie avec les résultats que donnent les coupes transversales et longitudinales des premiers stades embryonnaires, résultats que j'ai exposés dans le précédent chapitre. Nous avons vu, en effet, que, dès le stade D, la corde dorsale était déjà différenciée en avant du bourgeon caudal, et qu'ì la fin du stade $\mathbf{E}$, avant que le blastoderme ait atteint l'équateur de l'œuf, à un moment, par conséquent, où 
pour hupffer il n'existe pas encore d'embryon, on trouvait des protovertèbres formées de chaque còté du sillon médullaire, dans la région médiane de la prétendue ligne primitive. Il n'est pas du reste nécessaire de pratiquer des coupes pour constater l'existence de ces protovertèbres. Il suffit d'examiner par transparence un embryon de la fin du stade $\mathrm{E}$, détaché du vitellus,coloré et monté dans le baume dı Canada, pour apercevoir nettement les somites sous forme de masses rectangulaires bien différenciées. Cette simple observation suffit, je crois. pour réduire à néant l'hypothèse de Kupffer.

On sait, en effet, que chez les Mammifères et les Oiscanx, où la ligne primitive est bien développée, et mème chez les Reptiles, où cet organe existe à l'état rudimentaire 'd'après Balfour (9) et Strahl (181-184), les protovertèbres n'apparaissent que dans la région embryonnaire, et qu'il ne s'en forme jamais au niveau de la ligne primitive, tant que celle-ci reste visible, et n’est pas englobée par la partie postérieure de l'axe nerveux. Il en est de même de la corde dorsale, qui, chez ces mêmes animaux, n'existe jamais qu'en avant de la ligne primitive. A moins d'admettre que l'embryogénie des Poissons osseux diffère complètement de celle des autres Vertébrés, et que chez des animaux, des somites peuvent se développer de chaq ue côté de la ligne primitive, avant mème la formation de la tête de l'embryon, on est obligé de reconnaître que líupferer, entraîné par des vues théoriques, a commis une grossière erreur d'observation. Cette erreur est d'autant moins explicable, qu'on doit ì cet habile embryogéniste des découvertes très importantes, relatives au développenent des Vertébrés.

Si hupffer s'est trompé dans l'interprétation des premiers stades dı développement, est-ce à dire que toute sa théorie doit être rejetée ? Je ne le crois pas, et j'établirai plus loin qu'on peut admettre, chez les Poissons osseux, l'existence d'une ligne primitive rudimentaire ayant avec l'embryon les mêmes rapports que chez les Vertébrés supéricurs. Mais avant d'exposer les faits en faveur de cette hypothèse, il convient de considérer le développement général du système nerveux.

Le sillon longitudinal qui apparaît à la surface de l'écusson embryonnaire, sur la ligne médiane, correspond bien, comme l'admettent tous les auteurs, sauf liupferer, à la gouttière ner- 
veuse des autres Vertébrés. Ce qui caractérise les Téléostéens, comme les Cyclostomes et le Lépidostée, c'est que cette gouttière disparait de bonne heure par un processus spécial. Les bords du sillon ne se rapprochent pas par leur partic supérieure, pour former un canal, ni ne s'accolent pas par leur face interne pour constituer une fente linéaire, comme l'a dit le premier Calberla; ils se rapprochent par leur partie profonde, de sorte que le fond de la gouttière est soulevé et arrive finalement au même niveau que les bords. Il y a done là évagination plutòt qu'invagination.

Lorsque le sillon longitudinal prend naissance au stade C, il a la forme d'un $V$ dont la pointe est en contact avec le bourgeon caudal. Peu à peu ses bords se rapprochent, deviennent parallèles et se fusionnent à la partie antérieure de l'embryon; puis le sillon devient de moins en moins profond et disparait de la partie caudale à la partie céphalique de l'embryon. Les coupes transversales de ces différents stades, montrent bien le rapprochement des bords du sillon et l'épaississement progressif de l'ectoderme sur la ligne médiane. Elles montrent en outre que les rangées de cellules ectodermiques, disposées primitivement suivant des courbes à convexité inférieure, se redressent de la profondeur vers la surface et finissent par former des courbes à convexité supérieure. Pour comprendre le processus de l'effacement de la gouttière médullaire, on peut comparer les bords du sillon à deux vagues qui, poussées l'une contre l'autre, se fusionnent par leur base sans déferler, pour constituer une vague unique. La vague résultant ainsi de la fusion des deux autres est l'axe nerveux, qui est constitué alors par une masse celhulaire pleine, présentant à sal partic supérieure un sillon peu profond et étroit, reste du sillon primitif; ce sillon disparait complétement au stade $\mathrm{F}$ ou an stade $\mathrm{G}$.

Je ne pense pas quon puisse admettre, avec Goronowitsch, la formation successive de deux sillons. Le second sillon qui, d'après cet anteur, résulterait d'une invagination de la couche superficielle de la lame médullaire, n’est que le sillon primitif rétréci.

Sur des embryons durcis par l'acide elromique, le sillon médullaire rétréci, aux stades $\mathrm{D}$ et $\mathrm{F}$, parait beaucoup plus profond que lorsqu’il est encore largement étalé an stade C. Cette 
apparence est due à une altération des tissus embryonnaires par le réactif durcissant. Ainsi que je l'ai déjà dit, les cellules embryonnaires se multiplient activenent sur la ligne médiane de l'embryon, au point où l'ectoderme s'épaissit pour constituer la carène nerveuse. Cette prolilération cellulaire se traduit par la présence de nombreuses figures eytodiérétiques, principalement à la partie supérieure de la lame médullaire. L'acide chromique détruisant en grande partie ces cellules en voie de cytodiérèse, il en résulte un effondrement au fond du sillon qui parait alors beaucoup plus profond qu’il n'est réellement. Goronowitsch a fidèlement représenté l'aspect des coupes pratiquées sur des embryons trailés ainsi par l'acide chromique. Les cellules vésiculeuses qu’il signale sur le plancher du sillon médullaire sont précisément des cellules en cytodiérèse. Quand on traite les embryons par lacide osmique ou le liquide de Kleinenberg, tous les éléments cellulaires sont bien conservés, et le sillon médullaire est, dans ce cas, à peine indiqué sur les coupes transversales.

C'est aussi la destruction des cellules superficielles de la lame médullaire qui fait que la lame enveloppante semble passer comme un pont d'un bord du sillon à l'autre. Les cellules de cette lame résistent, en effet, bien mieux à l'action de l'acide chromique que les cellules embryonnaires; ce sont des éléments différenciés depuis longtemps, ayant déjà subi un commencement de kératinisation. Sur les embryons bien fixés, j’ai toujours vu la lame enveloppante exactement accolée à la surface de l'ectoderme, et tapisser le fond du sillon médullaire, comme l'avait figuré OEllacher.

J'ai indiqué, en décrivant la forme extérieure des embryons, l'apparition à la surfice de l'axe nerveux d'un sillon transversal et de fossettes dont jaai donné la signification; ces productions, toutes superficielles et accusées sur les embryons durcis par lacide chromique, de la mème manière que le sillon longitudinal, représentent les vésicules cérébrales primaires. L'axe nerveux est alors très large et très épais à la partie antérieure de l'embryon. Il se rétrécit et s'amincit de plus en plus d'avant en arrière.

Le système nerveux, comme du reste tout l'ectoderme, est toujours, sauf en avant du bourgeon caudal, nettement délimité 
des feuillets sous-jacents. Telle n'est pas l'opinion d'OEllacher, qui admet, ainsi que nous l'avons vu, qu'au début les feuillets externe et moyen, ou plutôt la partie inférieure du système nerveux et la corde dorsale sont confondues dans toute la Jongueur du cordon axial. J'ai montré, en parlant des coupes des stades C et D, que le cordon axial était traversé, en avant du bóurgeon caudal, par une ligne qui est le vestige de la cavité germinative. OEllacher n'a pas vu cette ligne et a été induit en erreur par la disposition caractéristique des cellules embryonnaires en couches concentriques, l'une des moitiés de chaque cercle se trouvant dans l'axe nerveux, l'autre dans le feuillet inférieur. Cette disposition résulte du mode de formation de l'endoder'me primaire.

Avant la formation de l'écusson embryomnaire, dès le stade A, l'ectoderme est épaissi sur la ligne médiane du futur embryon. Les cellules, en ce point, sont disposées en couches superposées, décrivant des ares de cercle ì convexité supéricure, et dont le centre est l’axe même de l'embryon. Cette structure se retrouve au bord du bourrelet blastodermique, où se produit la réflexion de l'ectoderme.

Dans la couche réfléchie, les cellules conservent la même disposition, mais alors les couches en are de cercle sont renversées et ont leur convexité dirigée vers leur vitellus. Les arcs de cercle de l'endoderme primaire et ceux de l'ectoderme ont une base commune qui est la cavité germinative, réduite à une cavité virtuelle. Liensemble de ces deux formamations, symétriques par rapport au plan horizontal qui sépare l'ectoderme de l'endoderme, constitue le cordon axial.

La symétrie des deux moitiés du cordon axial persiste jusqu'à la différenciation de la corde dorsale. A ce moment la moitié inférieure devient un cordon dont les cellules changent d'aspect et se groupent d'une manière particulière, mais la moitié supéricure conserve longtemps, dans l'axe nerveux, sa stucture primitive.

L'axe nerveux n'est d'abord qu'un épaississement de l'ectoderme et il est impossible de déterminer, sur des coupes transversales ses limites latérales. A mesure que l'épaississement s'accentue, sur la ligne médiane de l'embryon, pour constituer la carène nerveuse, il devient plus facile de distinguer dans le 
feuillet externe ce qui appartient à l'axe nerveux et ee qui forme l'ectoderme proprement dit. Mais cette distinction n'est possible que pour la partie inférieure du système nerveux, qui pénètre comme un coin entre les deux masses mésodermiques. La partie supérieure de l’axe nerveux est encore, en effet, largement unie à l'eetoderme et il n'existe ancune limite entre les cellules ectodermiques et les futures cellules nerveuses.

C. K. Hoffmann (89) déerit cependant et figure, sur des coupes correspondant à peu près aux stades $\mathrm{E}$ et $\mathrm{F}$, une couche ectodermique bien nette, formée d'une seule rangée de cellules, située au-dessous de la couche enveloppante de eellules, et s'étendant à la surface des masses mésodermiques et au-dessus de l'axe nerveux. Cette eouche serait la couche fondamentale de l'épiderme; ses eellules appartiennent au type de l'épithélium eylindrique tandis que les eellules de l'axe nerveux sousjacent sont aplaties. Leur grand axe étant dirigé parallèlement à la surface de l'embryon et perpendiculairement àl'axe de celui-ci.

Les meilleures coupes du stade $F$ sont loin de donner des inages aussi nettes que celles qui ont été représentées par Hoffmann. Au-dessus de la couche enveloppante, à la partie supérieure de l'axe nerveux, on trouve bien des cellules moins aplaties que les eellules profondes, mais il existe toutes les transitions possibles entre ces dernières et les premières. A ce stade, l'axe nerveux n'est done pas encore séparé de l'eetoderme, dans sa partie supérieure et médiane. Mais la séparation est en train de s'effectuer sur les côtés de l'axe. (Planehes, fig. 95.)

De chaque côté de la earène nerveuse, au point où celle-ci s'enfonee entre les lames mésodermiques, on voit une ligne claire qui part de la face inférieure de l'ectoderme et qui s'avance de bas en haut et de dehors en dedans, vers le plan médian de l'embryon. Cette ligne courbe à eonvexité externe s'arrête à deux ou trois rangées de cellules de la surface supérieure de l'ectoderme. Elle sépare les cellules aplaties de l'axe nerveux des cellules eetodermiques polygonales. En dehors de la ligne claire existe, dans la région du cerveau postérieur, un épaississement eetodermique correspondant à une petite dépression superfieielle. Les cellules de cet épaississement, qui deviendra la vésieule auditive, sont disposées en trois couches. Les cellules de la couche profonde sont allongées, à grand axe perpendiculaire à 
la surface del'ectoderme, et dirigées vers le fond de la dépression superficielle; les cellules des deux autres conches sont plus petites, polygonales et irrégulièrement disposées. La vésicule auditive est en continuité, dans sa partie externe, avec la couche épidermique de l'ectoderme formés d'un seul rang de cellules (Planches, fig. 95 va).

Les lignes de séparation de l'ectoderme et de l'axe nerveux s'avancent à la rencontre l'une de l'antre au-dessous de la rangée superficielle des cellules ectodermiques et, lorsqu'elles se sont rejoint sur la ligne médiane, le système nerveux central est alors séparé et nettement différeneié de l'ectoderme. Cette séparation définitive ne s'observe qu'au stade G et non aux stades précédents, comme le veut Hoffmann.

La séparation du système nerveux central de l'ectoderme a lieu d'abord dans la région céphalique et se continue davant en arrière, jusqu'au niveau de la vésicule de Kupffer. J'ai déjà décrit, à propos du stade $H$, les rapports que présentent les différents feuillets en avant, au milieu et en arrière de la vésicule de kupffer. J'ai montré que la corde dorsale ne commence à se différencier de l'endoderme primaire qu'en avant de la vésicule, que celle-ci est contenue dans l'épaisseur du feuillet interne, et qu'au-dessus d'elle l'ectoderme est immédiatement en contact avec l'endoderme. Si l'on compare les coupes de cette région (Plinches, fig. 102 et 107) à des coupes pratiquées a travers la partie postérieure d'un embryon d'Oiseau (Oie, Perroquet) ou de Reptile (Lézard), dans la région du canal neurentérique, on est frappé de la ressemblance qui existe dans la disposition des feuillets blastodermiques. On ne trouve plus en ce point de limite nette entre l'ectoderme el l'endoderme, et la seule différence entre les Téléostéens et les Sauropsides, c'est que chez ces derniers il existe une communication entre le tube digestif et le canal médullaire, tandis que, chez les Poissons osseux, laxe nerveux étant primitivement solide, il ne s'établit entre cet axe et l'intestin qn'une simple relation de contact. Nous verrons, en effet, que la vésicule de Kupffer est le premier vestige de la partie postérieure de l'intestin moyen.

La région de la vésicule de Kupffer correspond donc chez les Poissons osseux à la région du canal neurentérique des Verté- 
brés supérieurs. Déjà Balfour (9) avait remarqué qu'à l'extrémité postérieure de l'embryon du Lépidostée, la partie axiale de l'endoderme, la corde dorsale et le cordon ectodermique qui représente l'axe nerveux, se confondent, et il avait assimilé le produit de leur fusion au canal neurentérique de l'Amphioxus, des Plagiostomes, des Amphibiens, des Sauropsides et des Hammifères. C. K. Hoffmann admet anssi cette analogie pour les Poissons osseux bien qu'il n'ait pas constaté l'existence de la vésicule de kupffer chez les Salmonides, ni, dit-il, de véritable canal neurentérique.

S'il n'y a pas chez les Téléostéens, de véritable canal faisant communiquer lit cavité de lintestin avee celle du système nerveux central, e'est que cette dernière n'existe pas et ne se forme que tardivement. Elle n'apparait que vers la fin du stade $\mathrm{G}$ et dans la partie antéricure de l'embryon seulement; dans la partic postérieure, surtout au niveau de la vésicule de Kuppfer, la cavité du système nerveux ne se forme qu'après la fermeture du blastoderme.

OEllacher (123) admet que la cavité de l'axe nerveux, le canal médullaire, résulte d'une destruction des cellules centrales de cet axe. Ces cellules subissent une sorte de liquéfaction et sont résorbées; à leur place on voit apparaitre une cavité irrégulière qui plus tard se régularise pour constituer le canal de l'épendyme. Telle est aussi l'opinion de C. K. Hoffmann. (89) Dans les premiers stades du développement, dit-il, on remarque que les noyaux des cellules du système nerveux se colorent également bien, et que le protoplasma lui-mème se colore plus ou moins. A un stade plus avancé, lis partie centrale de l'axe nerveux ne prend plus de coloration sous l'inflence des réactifs. Avec un fort grossissement on constate une masse finement granuleuse, difficile à décrire, dans laquelle apparaît plus tard une fente. Cette fente provient d'une fluidification de quelques cellules placées sur la ligne médiane.

Pour Schapringer (165) et Weil (195) la cavité du système nerveux résulte d'un simple écartement des cellules situées dans le plan médian. Calberla (31) adnet un processus identique; mais comme, suivant lui, il se produit an fond du sillon médullaire une invagination, sous forme de pli, de la couche enveloppante, ce sonț les deux moitiés du pli qui s’é- 
cartent plus tard et laissent entre elles le canal central du système nerveux.

Ziegler (200) décrit la formation de la eavité d'une manière un peu diffẻrente. Les cellules aplaties, qui limitent de chaque côté la carène nerveuse, s'accroissent, et la carène tend à s'allonger. Mais cet allongement ne peut se faire ni vers le bas à cause de la corde dorsale, ni vers le haut à cause de la soudure des deux bourrelets médullaires qui se sont réunis sur la ligne médiane. Les couches externes de l'axe nerveux sont donc obligées de s'infléchir en dehors de chaque côté de la ligne médiane; en s'écartant ainsi elles donnent naissance à une cavité centrale.

L'hypothèse d'OEllacher et de Hoffmann repose sur une altération des tissus par les réactifs. La description qüils donnent est parfaitement exacte pour les embryons fixés et durcis par l'acide chromique; mais on n'observe jamais la destruction des cellules centrales de l'axe lorsque l'embryon a été fixé par l'acide osmique ou l'acide picrique. Ces cellules sont pour la plupart en cytodiérèse et ne résistent pas à l'action de l'acide chromique, ainsi que je l’ai déjà dit à plusicurs reprises; elles se réduisent en une sorte de masse informe, granuleuse, qui ressemble à un liquide coagulé remplissant une cavité. L’explication de Ziegler est aussi inadmissible ; cet auteur a été, ainsi que les précédents, induit en erreur, par sa technique. Ce ne sont pas, en effet, les cellules de la eouche extérieure de la carène qui s'accroissent et se multiplient le plus activement, ce sont au contraire les cellules centrales, comme le prouvent les nombreuses figures cytodiérétiques qui s'observent dans cette région.

La cavité ecntrale du système nerveux apparaît d'abord comme une simple ligne sur le plan médian de l'embryon; elle résulte, comme l'avaient bien vu Schapringer et Weil, d'une séparation des cellules.

Ce sont les cellules filles des cellules en roie de division sur la ligne médiane, qui s’écartent les unes des autres, laissant entre elles une cavité virtuelle qui ne devient réelle que plus tard, lorsque, par suite d'un accroissement plus rapide des parties centrales, les deux moitiés de l'ax̧e nepeux šinfléchissent extérieurement vers les plaques mésodermiques, la flexion en 
dehors est due à la tension qui existe dans chacune des faces de la fente par suite de la multiplication des cellules. Ziegler a raison de faire joner un róle à la corde dorsale et à la partie supéricure de l'cmbryon, qui, agissant comme deux plans résistants, s'opposent à l'allongement en hauteur de l'axe nerreux. Celui-ci est comparable à un tube en cantchouc, qui, comprimé entre deux surfices rigides, et sounis à une pression interne, ne pent augmenter de diamètre que parallèlement aux deux surfaces. Mais Ziegler me paraît être dans l'erreur lorsquil admet que la tension existe an niveau des couches externes de la cavité nerveuse.

Dans la région médullaire proprement dite de l'embryon, la cavitè ne se produit que sur la ligne médiane et s'arrête à une certaine distance de la partie supérieure et de la partie inférieure de la moelle; dans la région céphalique, dans les vésicules cérébrales, la fente se termine inférieurement par un espace triangulaire dont la base, légèrement convexe en dedans, est parallèle au vitellus; supérieurement elle se divise en deux branches qui se dirigent de dedans en dehors en décrivant une courbe à convexité supérieure. Ces deux fentes latérales séparent de la portion dorsale de laxe nerveux une rangée de cellules qui forme le toit des vésicules (Planches, fig. 104).

Jusqu’à la fermeture du blastoderme, le système nerveux ne subit pas d'antres transformations. Ce n'est que plus tard que se différencient les parties du cerveau dont le développement a été bien étudié par Rabl-Ruckhard. (131.)

Bien qu'il ne rentre pas dans le cadre de mon travail de m'occuper des stades ultérieurs à la fermeture du blastoderme, je rappellerai cependant une observation importante faite par Kupffer, parce qu'elle présente un grand intérêt au point de vue de l'embryogénie générale.

Kupffer (106) a signalé, sur des embryons de Truite de 19 jours, plusieurs jours après la fermeture du blastoderme, une segnentation transversale de la moelle allongée. On peut compter cinq segments : le prenier est immédiatement cn arrière de l'ébauche du cervelet, le dernier correspond à la partie postérieure des vésicules auditives. Ces segments sont nettement visibles extérieurement à la surface de la moelle; plus tard ils 
s'effacent, mais on les retrouve sur les coupes horizontales ou longitudinales.

Je puis confirmer entièrement l'observation de Kupffer. J'ai constaté sur les embryons des stades qu'il indique, et même sur des embryons moins avaneés, dès le stade $\mathrm{G}$ et avant la fermeture du blastoderme, l'existence des cinq segments au niveau de la moelle allongée. Sur des coupes longitudinales, les segments de l'axe nerveux ont tout à fait l'apparence de protovertèbres. Ils se présentent sous la forme de rectangles allongés dans le sens de la hauteur de l'embryon, exactement contigus et occupant toute l'épaisseur de la moelle. Dans chacun de ces métamères, les cellules ne présentent pas de caractères particuliers et ne sont pas disposées en une couche régulière à la périphérie, comme dans les métamères mésodermiques (Planches, fig. $86, m n)$.

C'est à Kupffer que revient le mérite d'aroir appelé l'attention des embryogénistes sur cette segmentation d'une portion de laxe nerveux chez l'embryon. Avant lui, cependant, plusieurs auteurs avaient observé cette disposition, mais sans en eomprendre l'importance. C'est ainsi que Baer (5) dit que chez le Poulet, au troisième jour, chaque lame de la moelle allongée ıorme plusieurs plis courts, et quau quatrième jour ces plis forment des stries transversales distinctes. Bischoff (23) a représenté planche $\mathbf{X}$, figure 41 , une coupe longitudinale de la partie céphalique d'un embryon de Chien de $2 \%$ jours, sur laquelle on voit à la face interne du cerveau postérieur sept plis irréguliers; mais eet auteur ne parle pas de cette disposition dans son texte. Remak (146) décrit également, chez le Poulet, du troisième au cinquième jour, sur les parois du cerveau postérieur, einq à six masses carrées présentant une grande ressemblanee avec des protovertèbres, et dont il ignore la signifieation. Dursy (40) (pl. III, fig. 14) figure, à la face interne du quatrième-ventricule d'un embryon de Vache de 65 cent., six plis semblables à ceux que Bischoff a vus chez le Chien; de même que ce dernier auteur, Dursy ne signale pas ce détail dans son ouvrage. Gutte (59), llans son Atlas, représente la segmentation dı quatrième ventricule chez une larve de Bombinator (pl. VIIl, fig. 131). Dohrn (37) parle de huit ì neuf renflements ganglionnaires qu’on voit distinctentent au niveau du quatrième ventri- 
cule des embryons de Poissons, notamment de la Perehe, et il assimile ces renflements aux ganglions de la chaine nerveuse d'un Articulé. Kiolliker (97) (fig. 339 et Scessel (174) (pl. XXI, fig. 2) représentent, le premior sur un embryon de Lapin de 10 jours, le second sur un embryon de Poulet de 3 jours, la segmentation de la moelle allongée.

Balfour, dans son Traité d'embryologie (p. 349, édition anglaise de 1881), signale en ces termes la métamérie du cervean postérieur : "Sur les còtés se développe, chez le Poulet, une série de constrictions transversales qui lo divisent en lobes dont le nombre ne parait pas constant. Le plus antérieur de ces lobes persiste, et sa roûte devient le cervelet. On ignore si d'autres étranglements ont une signification moryhologique quelconque. Il s'en produit de plus ou moins semblables chry les Téléostéens. A une époque plus avancée, la noelle allongée présente à sa surface interne, chez les Elasmobranches, une série de lobes qui correspondent amx racines des nerfs vague et glosso-pharyngien, et il se pent que les étranglements plus précoces correspondent naturellement à autant de racines nerveuses ".

Béraneck (21) décrit avec soin et figure dans la planche XXIX de son mémoire, cunq replis médullaires de chaque cóté du cerveau postérieur, chez de jemnes embryons de Lacerta agilis, et il ajoute que éest de la première paire de ees replis que part le nerf trijumeau, et que c'est de la troisième paire que part la racine du facial et de l’auditif. Le méne auteur (22) a étudié aussi les replis médullaires chez le Poulet. Enfin dans ,une note récente, Kupffer (107) dit aroir retrowvé la segmentation de la moelle allongée en cinq métamères, sur un cmbryon d'Épinoche, sur un embryon humain de trois semaines environ, et sur des embryons de Ilouton et de Souris. Il a constaté aussi l'existence de deux ou trois métamères dins le cerveau moyen, en avant des métamères de la moelle allongée. Chez de jeunes embryons de Salamandra atra, avint la fermeture du blastopore et l'apparition des protovertibres, il a reconnu que l'ébauche du cerveau présentait huit paires de segments, et que la segmentation se continuait dans la moelle jusqu'au voisinage du blastopore.

J'ajoutcrai que, sur des coupes longitudinales d'embryons de Lapin de 10 et 12 jours, jai observé aussi trìs nettement les 
cinq renflements du cerreau postérieur, mais que je n'ai pas vu les métamères du cerveau moyen.

Il résulte du court historique qui précède qu'on doit admettre aujourd'hui que, chez tous les Vertébrés, le système nerveux central présente, à un certain moment de son développement, une segmentation transversale régulière. Cette segmentation parait être surtout localisée au niveau de la vésicule cérébrale postérieure; mais elle peut s'observer aussi dans le cerveau moyen et dans la moelle épinière (Salamandre). On doit se demander, avec hupffer, si cette métanérie est un vestige d'une segmentation primaire de l'ixe nerveux, qui existait chez les formes ancestrales des Vertébrés et indique une communauté d'origine avec les Articulés, ou si c'est seulement une disposition secondaire en rapport avec l'origine de certains nerfs.

ll serait, je crois, prématuré de choisir entre ces deux hypothèses. Si l'observation de hupffer sur la Salamandre prouve que les métamères nerveux apparaissent de très bonne heure, chez les autres Vertébrés nous les voyons se produire assez tardivement et dans une région limitée de laxe nerveux. Chez la Truile, j’ai constaté l'existence des métamères dès le stade $G$, c'est-ì-dire à un stade précoce, mais au nombre de cinq seulement. Il est done nécessaire d'examiner avee plus de soin les premiers stades du développement des Vertébrés et de rechercher si les segments se forment de honne heure et dans toute l'éteudue du systène nerveux, et si ceux de la région de la moelle allongér ne sont que des métamères plus différenciés, et persistant plus longtemps que les autres, par cela même qu'ils sont l'origine de nerfs importants. A l'appui de cette manière de voir, qui, si elle était confirmée, derrait faire admettre la métamérie primitive du système nerveux, il convient de rappeler que chez certains Poissons, Trigles, Baudroie, Mòle, les segments de lis moelle allongée persistent à l'âge adulte et constituent ce que les auteurs ont désigné depuis longtemps sous le nom de lobes accessoires. Ussow (189), qui a étudié avec soin la structure interne de ces lobes, a vu que chacun d'eux correspond à une disposition particulière des élénınts nerveux, qui indique bien qu'il existe chezces Poissons une mètamérie de cette région du système nerveux. 
Organes des sens. - Tésicules optiques. - Le déveloplement des yeux ne présente chez les Poissons osseux aucune particularité remarquable. Comme chez tous les autres Vertébrés, les vésicules optiques sont une dépendance du cerveau antérieur et s'en différencient par un processus identique, et à une périorle plus avancée du développement. L'évolution de ces organes a été hien suivic par les anciens embryogénistes et plus récemment par Schenk (166), OEllacher (123) et C.-K. Hoffmann (90). Je n'ai pu que confirmer les observations de ces auteurs.

Le système nerveux central des Poissons osseux étant, au début, une masse solide sans cavité, les vésicules optiques ne sont aussi primitivement que des expansions solides de l'axe. Chaque vésicule apparait comme un épaississement latéral mal délimité, à base très large. Cette base se rétrécit de plus en plus, à.mesure que l'épaississement augmente, de sorte qu'au stade G, la vésicule est constituée par une masse ovoïde, rattachée par un pédoncule au cerveau antérieur. L'étranglement de la base de la vésicule optique ne se fait pas d'une manière uniforme sur tout le pourtour de celte vésicule : il marche plus rapidement de haut en bas, et d'arrière en avant; il en résulte que le pédoncule, oul le nerf optique, est d'abord situé à la partie antérieure et inférieure de la vésicule.

Lal cavité de la vésicule optique se forme à peu près à la même époque et par le même procérlé que celle du système nerveux central, par séparation de cellules, surtout de cellules en cytodiérèse. Cette cavité apparait soms forme d'un croissant à concavité interne, vers la partie interne de la masse pleine de la vésicule; on l'observe déjà quand il n’y a pas encore de fente dans le cerveau (Planches, fig. 99, ro). La l'ente interne do la vésicule s’étend de proche en proche dans le pédoncule pour se mettre en rapport avec la cavité centrale du cerveau antérieur.

Lorsque la résicule n'est plus rattachée au cerveau que far' 1111 étroit pédoncule, elle a clıngé de forme; đovor̈de qu'elle était, elle est devenue discö̈le; elle s'est aplatie parallèlement au plan longitudinal et vertical de l'embryon. En même temps elle s'est accrue de bas en liaut. II se produit probable- 
ment dans son intérieur un déplacement de cellules. La paroi de sa face externe šépaissit, en effet, tandis que la paroi de sa fice interne s'amincit et n'est plus constituée que par une seule couche de cellules. La cavité est alors tout à fait excentrique et très approchée de la partic proximale. A ce stade, on distingue nettement dans la vésicule une couche externe épaisse qui est lorigine de la rétine, et une couche interne mince qui sera la zone pigmentaire.

La vésicule optique, primitivement en contact avec le cerveau, s'en sépare par suite de l’interposition d'une couche de tissu mésodermique: mais ello reste toujours en rapport, dans sa partie inférieure, avec liaxe norveux par son pédicule, qui subit un déplacement et se rapproche de la partic postirieure de la vésicule. Dès la fin du stade G. l'ectoderme commence à s’épaissir au niveau de la vésicule optique: ses rellules se multiplient et se disposent en deux ou trois couches sur une certiane étendue. La lame enveloppante ne prend pas part à cette formation qui est l'ébanche du cristallin. L’épaississement ectodermique, d'ibord mal limité et recouvrant presque entièrement la liace distale de la vésicule, se concentre vers le nilieu de cette face; il constiue alors un hourgeon qui refonle la couche rétinieune et sera plus tard enveloppé par elle (Planches, fig. 103, cr). An moment de la fermeture du blastoderme, la vésieule optique sn présente, soit sur des coupes transiersales, soit sur des coupes horizontales sous la forme dim haricot dont le hile correspond au cristallin.

Liœil des Tóléostéens présente, dans son développement, les mèmes plaises que le système nerveux central : la vésicule optique est d'abord me masse solicle qui se ereuse ensuite d'une cavité : la couche rétinionne ne provient pas comme chez les autres Vertébrés, d'ume invagination de la face distale vers la like proximale; elle rósulte d’une simple délamination. 11 en en est de même du cristallin qui se forme, non par une invagination, mais par un épaississement solide de lectode rme. Il fant aussi remarquer que, de mème que chez les Amplibiens et le Lépidostée, la lame enveloppante reste étrangère ì la constitution dn cristallin, ainsi que Schenk l'arait dejì hien itabli. 
Tésienles auditives. - Las résienles audilires apparaissent presquien ineme temps que les vésicules optiques. Lemr place est marquer reterienrenent par deux potites fossettes situés anx plrimités du troisième sillon longitudinal, au stade E. Sur des conpes tramsrersales, on conslate que, an niveau de ces fossettes, il existe, de chaque côté de la carène nerveuse, un épaississement dr la enuche ectodernique qui se continue encore sins ligne de démareation aree la partir sujérieure de laxe nerveux. Je ne pense done pas qu'on puisse dire arer. Hoffmann et Goronowitsch que les vésicules auditives ne proviennent que de la couche fondamentale de l'ectoderme et non de la lame médullaire, puisque, ainsi que je l’ai déjà dil, il est impossible de tracer les limites de colle-ei. C'est seulement par analogie, en so basant sur le développement des résieules auditives clece los autres Vertébrés, quon peut admetle que, chezles Téléostéens, ees organes ne proviennent pas du systime nopeus eentral.

Lépaississencut ectorlermique, première ébauche de la resicule anditive, est primitirument tout is liat an contact de la carène norveuse, au point oì dlle se détache de l'ectoderme. J'ali déjà déerit la disposition des rellules dans cet épaississoment, jajouterai que la lame mouvrante passe au-dessus de lui sans cutrer dans sa constitution. Bientít la résicule audilive se sépare de la couche ectodermique de lis même manière que laxe nerveux central : rlle constitue alors une masse

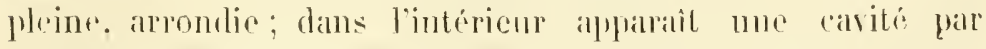
simple écartement des cellules; eetle cavité saigrandit assez rapidement. Au starle H, les parois de la vésicule sont encorr lomées de deux ou trois couches de cellules allongées disposées radialement (Planches, fig. 10' et 111, va).

Systrme nereur périphérique. - La question de lorigine des racines des nerfs périphériques et des ganglions spinaux ust lune des moins avaneées de l'embryogéne des Vertibrés. de ne moceuperai ici que du rleveloppenent des ganglions, frares quatu moment de la fermeture du blastoderme. les racines des netl's ne sont pas encore formées.

Mes observations confirment celles de Balfour (8), pour les 
Plagiostomes, de Marshall (119), pour le Poulet, ct surtout celles de C.-K. Hoffmann (89), pour les Poissons osseus. Le nerf anditif, ou tout au moins son ganglion, semble etrecelui qui apparait lo premier. Peu de temps apris la lormation de lat vésicule anditive, à la fin du stade F, lorśque la ligne de séparation de l'ectoderme et de laxe nervenx a atteinl environ la moitié de la distance qui la sépare de la ligne médiane, il se produit sur le còté du cerveau postérieur. nne seconde ligne, parallèlr ì la première et qui découpe, dans la partie supérieure de l'axe nerveux, une petite masse situce il la partie interne et inféricure de la résicule anditive (Planches, fig. 100, na). Lorsque le eerveau est complitement détaché de l'ectoderme, la petite masse, qui est l'origine du nerf auditif, reste en relation arec lui el en contact avec la résicule auditive; elle sallonge en bas et en dehors de manière à surve le déplacement de la vésicule auditive, lorsque celle-ci se sépare de l'ectoderme.

De petiles masses cellulates semblables se séparent en divers proints de laxe nerveus, dre chaque còlé de sa partie supérieure, par le mème processus qui donne naissance au nerf auditif.Ces protubérances sont l'origine des différents nerfs ċràniens et rachidiens, mais elles restent longtemps sans so développer, c'est-ì-dire quelles persistent sous forme d'un cordon cellulaire court raltaché ì la partic supérieure du système nerveux, et descendant entre ce systène el les protovertibres. Je nai plats suivi le déreloppement ultérieur de ces nerfs.

Les nerfs apparaissent done chez les Téléostéens de la méme manière que chez les Plagiostones. Il se produil de chaque côté le laxe nervens me séric d'exeroissances qui, par leur ensemble, constituent la crète neurale de Marshall. Chez les Plagiostomes (Balfour) (8), te Poulet (Marshall) (119), les Amphibiens (Bedot) (16), la crète neurale se forme, lorsque le sillon médullaire est fermé à la partic supérieure de l'ase nerveux; chez les Téléostéens, les exeroissances nervenses apparaissent lorsque l'axe nerrous est encore uni à l'ectoderme, et dans certains cas il est difficile de saroir si l'excroissance appartient à l'axe on à l'ectoderme. Les observations faites sur les Téléosféens paraissent étahlir un trait d'union entre la théoric de Balfour of celle de llis (86). Cet auterm ahmet que les ganglions nerveux naissent d'un repli ectodermique. lo sillon 


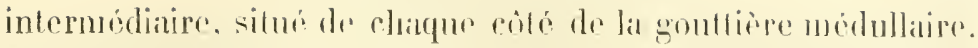
On peut, en effed, atmette que suivant les espèces amimales et suivant les diffirents nurfs. les ganglions apparaissent phus ou moins tit, at se développent soit aux dépens de lat lame ectodermique qui est enere en contimuti direte aver lase nerreux, soit aux dépens de laave nervemx lui-míme déjì diffórencié de l'ectoderme. Jo rappullerai que Harshall a conslaté que chez li Punlet, ertains nerfs cràniens appratrissent alvanl la fermeture de lis gontliere nervense, tamdis que bes nerfs spinaux apprarasisent après la fermeture dre cette gouttiere.

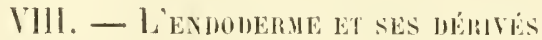

Liendoderme on fenillet interne, amx depens duquel so formera l'epithélimm du tube digestif atree ses dépendances, ne constitue pas an début un feuillet ayant lis mème valeur morphologique que l'actoderme. 11 n’ipparail, en effet, en lant pue feuillet diflérencié qu'on mème temps que lo mésorlerme. L'endoderme proprement dit, de mème que le fenillet moyen, est une formation secondare: le deuvieme fenillet primaige que jai designe soms le nom d'endoderme prinaire, llist qu'me formation temporaire et transitoir destinéc à se dédoubler en deus atutres fenillels.

Jai insisté longuement dans un précédent chappitre, sul lit formation de l'endoderme primaire, at indiqué l'opinion dis différents rmbryologistes sur ee sujot. Je rippellerai seulement que ce fruillet provient dun reflevion du bord libre de l'ectoderme dims lintérieur de lil cavité germinative "l que. chez certains Poissons, ainsi que l'a bien élabli II. ron Kun allewshi ((103) des eellules, provenant du pamblaste, viemnent sajouter aur cellules ectodermiques pour constituer l'endoderme printire.

Formo dabord uniquement par la partie réfléchie du bourrelet blastodermique, lendoderme prinaire, dans la lrogion embryonnaire, savanee de plus en phus dans linterieur do la cavite germinalive ì mesure que le foit de celle-ci samineit et que le germe s'étend ì lat surfite du vitellus. Des que l'eetoderme, réduit it une mince couche collulaire dans tonte

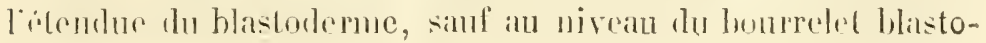


dernique of de l'écusson embryonnaire, s'est épaissi dans ces drux régions, de manière à constituer les parties opaques et saillantrs du blastoderme, on le trowe doublé dr l'endoderme primaire: c’est-it-diece que ce fruillet existe au niveau du bourrelet blastodermigur at de l'écusson embryonnaire.

Lindoderme primaire est formé d'un certain nombre de rangérs collulaires superposées, variant de trois à dix suivant les régions. En dérivant laspect des eompes transversiles et longitudinales des différents stades. jai fait connaitre les rarialions dépaisseur de ce feuillel, épaisseur qui est, en général, en raison inverse de celle de l'ectoderme.

Les cellules de l'endoderme primaire sont au début disposées sans order apparent, sauf sur la ligne mediane de l'emhryon, dans le cordon axial, où elles forment des conches coneentriques en demi-cerele, ainsi que je lai dit à propos de l'ectoderme. Vers la limite antérieure de l'embryon, et sur hes parties litérales, les cellules laissent entre elles des lacunes irrégulières surtont an contact du parablaste. Au stade D, les cellules de la couche supérieure de l'endoderme primaire $\therefore$ allohgent dans le sens verticil, deviennent prismatiques et constituent une conche de cellules en palissade analogue it celle déerite par OEllacher à la face inférieure de l'ectoderme. A cette époque, le deusieme feuillet primaire donne simultanénrut naissince atu mésoderme, à la corde dorsale et ì l'endoderme secon laire ou défnitif. Je considèrerai sucessive. ment clateune de ees formations.

Mésoderme. - En domnant l'historique des feuillets blastodermiques, j’ai indiqué les diverses opinions émises par les auleurs sul l'origine du mésoderme chezles Poissons osseux.

Les embryogenistes qui font provenir l'endorlerme du pariblaste considerent ce que jai désigné sous le nom d’endoderme primaire comme représentant le mésoderme. Tols sont Lerebonllet, Kupffer, Agassiz et Whitman, Klein. Van Bambeke, ron Kowalewsli; les uns regardant ee mésoderme comme résultant d'une simple différenciation de l'ectoderme, les autres comme produit par la réflexion de ce feuillet primaire.

Au contraire, les auteurs qui ne font jouer aucun ròle au parablaste dans la formation des feuillets, font en général déri- 


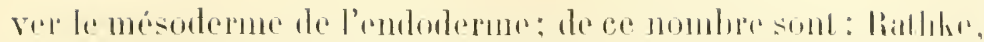

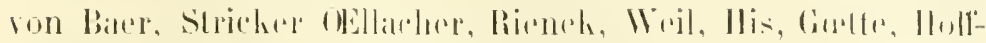

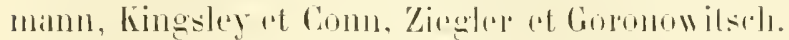

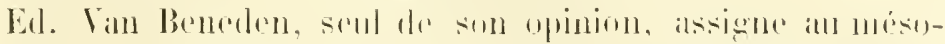

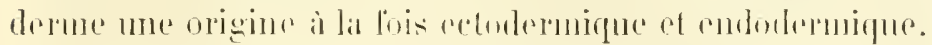

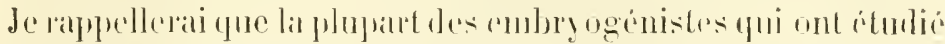

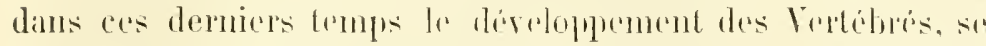

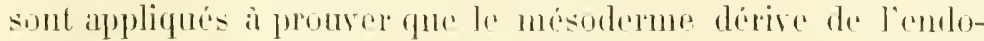
derme, et que les loeres llertwig 80 ont chrrelé à établir que le fenillet nogen provenait are peplis on länvaginations du fenillet interne, généralisint ainsi le proecsess de formation du troisieme fenillet obserré par how aldrshy chez l'A mphiones.

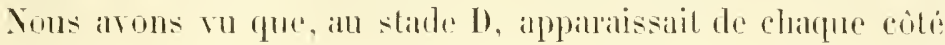
du cordon avial, en avint hu bourgeon candal, une liģne datre dans l'épaisseur de l'endoderme primaire. Cette ligne, correspomlant à une fente virturlle, progresse d'arriore en arant, ef

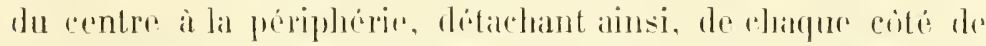
l'axe longitudinal de J'embryon, une lame pluricellutaire, qui est la lame mésodermique. Les denx lames mésodermiques sont séparées par la corde dorsale provenant du cordon axial. (Planches, fig. $110, \mathrm{~ms}$ ).

Le clivage de l'emboleme primare se poursuit ans stades $\mathrm{E}$ et $\mathrm{F}$, et finil palr alteinde le nivean de's résicules optiques: il sadecentue anssi en arrière jusqu'it lextremite posterieure de la vésicule de huptfor.

Les lames mesodermiques presentent leur masimm de largenr et d'épaisseur daus les portions postérienre ot mogrune de l'embryon; dans la région éphalipure elles sont étroites et formeses semlement de denx ou trois conches dre cellules. Lemp developlement est en raison inverse de celui de l'retoderme. Chatue lame remplit exactement l'espate romprit entre l'edoderme, la corde dorsile et l'endoderme secondarire: sil linome

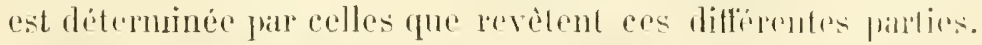

Dans les deux tiers posterieurs de l'enbryon, lil lanbe méso-

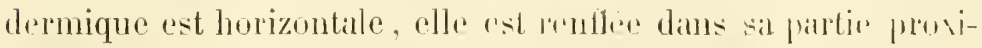
male et va en samineissant sur les burds du l'imbryon, nì ellenest plus constiture. romme les dems anlere linillets. que par une senle rangée de eellules. Som bord exlorme ast mal

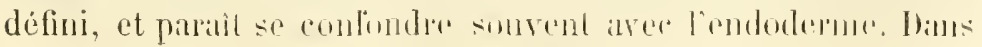




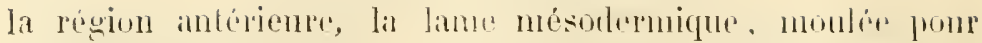
ainsi dire snr lat carène noprose, est flus épaisie dims sid partie moyenne qu'i ses extrémités proximale el distale. Dans la partic antérieure de la tète, il n’y a plus de lanes mésodermiques. Liendoderme primaire reste directement en contact aree le ststente norveux; mais ce feuillet n’a là aussi qu'une existence temporair'r. Lorsque la tète su soulère illdessus du vitellus de manière à derenir libre, cette partie de l'endoderme primaire se transforme entierement en tissu mésodermique.

Le mésoderme subit pendint son érolution des modifications très importantes que je décricii dans un chapitre spécial.

Corde dorsale. - La formation de lia corde dorsale marche parallèlement à celle des lamés mésodermiques.

Au stade D, le groupe des eellules du cordon axial se sépare de l'endoderme prinnaire par une ligne claire demicirculaire dont les deux extrémités rejoignent la ligne de séparation de l'ectoderme de l'endoderme primaire. Le cordon axial, ainsi différencié, devient la corde dorsale (Planches, fig. $110, c d)$. Celle-ci appariît en avant du bourgeon caudal et le processus de différenciation se continue darrière en avint. La lormation de la corde dorsale est lone, chez les Téléostéens, la mêrne que chez les autres Vertébrés. Tous les auteurs sont, en efiet, d'accord pour admettre que la corde dorsale se forme d'arrière en avant.

Les cellules de la corte dorsale conservent d'abord l'aspect et la disposition de celles du cordon atxial ; il en résulte que la corde dorsale a la forme d'un demi cylindre, situé au dessous de la rarène nerveuse. Miais plus tard les celhules se disposent concentriquement autour de l'axe même de la corde dorsale : cet organe devient alors un cordon cylindrique, mais toujour's un peu aplati au contact de la carène nerveuse.

La corde dorsale est toujours plus volumineuse it sa partie postérieure que dans sa partie antérieure, où elle se termine en pointe légérement infléchie vers le vitellus; elle représente dans son ensemble plutôt un cône très allongé qu'un véritable cylindre. Son volume diminue aussi pendant le développement 
de l'embryon jusqu après la fermeture du blastoderme, eomnte l'unt constaté Hoffmann (89), Ziegler (200) et Goronowitsch(57). A partir de celte éporjue elle angminte de volmme nais en changeant complètement d'aspect. Durant ers changements de volume, les déments to la eopdo dorsale subissent des transformations sur lesquelles les auteurs ne me partisisent pals avoid sulfisamment appelé l'attention.

Les collules, disposés en couches concentriques, sont primitivement légèrement aplaties comme dans le cordon avial, leur grand axe étant dans un plan vertical perpendieulaire à l'axe de linmbryon. Lorsque la corde dorsale commence it s'arrondir, les cellules deviennent polygonales at diminuent de nombre; rlles saplatiss'nt en mème temps dans le sens antero-postérieur. 11 resulte de eetle disposition que les conpes longitudinales if les coupes-transversales de l'organe ont un aspect tout a lait différent. Sur les premières, on voit des cellules allongées verticalenent el pressées les unes contre les antres, présentant une diszosition caractéristique, qui permet de reconnaitre facilement la corde dorshle au milien des autres tissus (Planches, fig. 108 et $109, c d)$; dans les secondes, on ne voit que des cellules polygoniles un pru plus grandes que les intres cellules embryonnaires.

Quand li corde dorsale diminue dr volume, anx stades Get H, l'aspect des conpes longitudinales n il pals changé, sauf que les collules sont rneore plus aplaties que préédemment; mais. dans les sections transversales, on ne compte plus qu'un tres petil nombre de grosses cellules, 3.6 on 7 . ragonnant autour diune cellule centrale, ou d'un centre virtuel. Le novau de ces eellules est périphérigne, at denx fois plus gros que celui des antres cellules d. l'embryon. Il paratit ainsi se produire une résorption de cellules pemdant l’évolution de l'organe; ce sont les cellules centrales at quelques unes des cellules péripheriques qui disparaissent. Que deviennent ces cellules? dispatraissent-elles véritablement"?

Dès que le cordon axial s'est différencié en corde dorsile. ses éléments cessent de se multiplier; on n'y trouve jamais, en effet, de cellules en voie de division; sur des centaines de coupes que j’ai examinées, c’est ì peine si j’ai rencontré une ou deux fois une figure eytodiérétique dans toute la longueur 
de la corde dorsale. Cet organe sallonge cependant en mème temps que l'embryon; son extrémité postérieure reste en rapport avec la vésicule de líuffer, son extrémité antérieure est ì peu près au niveau de l'extrémité antérieure des vésicules auditives. D’un autre còté, pendant cette augmentation de longueur, les cellules, sur des coupes longitudinales, restent toujours pressés les unes contre les autres, el semblent mème plus rapprochées; ces cellules ne se multipliant pas, on doit se demander comment peut se produire l'accroissenent lougitudinal de lorgane. 11 me semble très probable qu il se produit dans lit corde dorsale des déplacements de cellules, gui font que des cellules, comprises par exemple dans une section transversale, passent dans un plan antérieur on postérieur. Cette hypothese rend compte de l'allongement de la corde dorsale, alccompagné de la diminution de son épaisseur et dr laugmentation de vohme de ses éléments. Mais plus tard, quelque temps après la fermeture du blastoderne, lorsque la corde dorsale augmente de volume, on voit apparaitre des figures cytodiérétiques à sa périphérie. A ce moment la partie centrale de l'organe est occupée par de grands éléments irréguliers, remplis de larges vacuoles, et leurs noyaux se multiplient à la périphérie pour donner naissance aux petites cellules de la gatine.

La corde dorsale peut donc être considérée comme étant le premier organe de l'embrỵon, qui se différencie nettenent aux dépens des tissus embryonnaires et dont l'évolution est la plus rapide. Bien que cet organe napparaisse quaprès le système nerveur central, ses élémenls ont revêtu une physionomie tonte particulière et présentent déjà des phénomènes de régression, alor's que ceux du système nerveux ont conservé lous les caractères embryonnaires et ne se distinguent pas encore des cellules mésodermiques ou endodermiques. Ce fait démontre bien l'ancienneté phylogénique de la corde dorsale.

Les embryogénistes qui se sont occupés du déreloppement des Poissons: ont assigné à la corde dorsale les origines les plus diverses. Gatte (60) admet que, chez les Téléostéens comme chez les Amphibiens, cet organe provient du mésoderme ; c'est aussi à cette opinion que semble se ranger Salensky (163) pour le Sterlet. OEllacher (123) la fait provenir de la partie inférieure 
de sun cordon asial, qui, selon lui, résulte d'me fusion du mésoderme et de Jeetoderme. Radwamer (132) considere la corte dorsale comme provenant te l'ectoderme. A. Schultz (168), pour les Plagiostomes, pense qu'elle dérive d'une fusion de l'ectoderme et de l'endoderme. Enfin. Calberla (31) la fait se différencier de l'endoderme primaire chez les Salmonides et les Cyelostomes.

Lorigine endodermique de la corle dorsale parait avoir rallié la plupart des embryogénistes modernes, non seulement pour les Poissons, mais anssi pour les antres Vertébrés. Kowalevsky (101) et Ilatscheh (65) ont nettement étibli que chez l'Amphioxns la corde dorsale est formée par un repli de l'endoderme. Ballour (8) a vu chez les Plagiostomes la corde dorsale dériver de l'endoderme; e'est au mème résultat que sont arrivés Scott (172) et Shipley (198) jour les Cyclostomes, Kingsley et Conn (98), Hoflimam (89), Ziegler (200) et Goronowilsch $\mathbf{5 7}$ pour les Joissons osseux. Wes propres recherehes confirment celles de ces dernicrs auteurs et démontrent que la corde dorsale provient de l'endoderme primaire. Cet organe se sépare de lendoderne primaire en mème temps que les plaques mésodermiques, il a donc lat mème valeur morphologique que ees dernières, et on peut le considérer comme représentant la partie nuédiane dı mésoderme, ainsi que l'avait bien vu Calberla. La formation simultance de trois replis endoderniques, chez l'Amphioxes, dont le médian devient la corde dorsale et les deux lateraux sont lorigine du mésoderme, prouve que, chez les Téléostéens, le développement de ces parties suit me marehe identique; mais, chez ces animaux, ce sont des masses cellulaires pleines qui se séparent de l'endoderne, tandis que chez l'Amphioxus, le mésoderme et lis corde dorsale sont des éviginations crenses du feuillet interne.

Endoderme proprement dit. - Lorsque la corde dorsale et les plaques mésudermiques se sont séparées de l'endoderne primaire, il reste en contict arec le parablaste une couche cellulaire qui est l'endoderme définitif ou secondaire et qui devient l'épithélium de la cavité digestive.

Suivant OEllacher (123) l'endoderme est formé de deux ou 
trois couches de cellules. Cette constitution de l'endoderme serait, d'après lui, un des caractères qui distinguent les T'éléostéens, ou tout au moins la Truite, des autres Vertébrés, chez lesquels le feuillet interne est toujours réduit à une seule couche de cellules. Hoffmann (89) admet, all contraire, que l'endoderme ne comprend, chez les Salmonides, qu'une couche unique de cellules fusiformes et que cette couche ne s'étend pas aussi loin latéralement que les lames mésodermiques. Ziegler (200) dit que le feuillet interne consiste en une couche unicellulaire ou pluricellulaire qui s'étend sur toute la lungueur de l'embryon. Goronowitsch (57) partage la même opilition.

La constitution de l'endoderme secondaire varie suivant le stade du développement embryonnaire auquel on le considère, et aussi suivant la région de l'embry̧on. Au moment de la séparation de la corde dorsale et des lames mésodermiques, l'endoderme secondaire est formé de trois ou quatre couches de cellules et s’étend sur toute la largeur de l'embryon. Le nombre de ces conches va en diminuant aux stades suivants, surtout sur la ligne médiane de l'embryon; il se produit en mème tempsune sorte de coneentration vers la ligne médiane, qui fait que le feuillet interne ne tapisse plus inférieurement les extrémités distales des lames mésodermiques, qui reposent alors directement sur le parablaste. L'observation de Hoffmann est en cela parfaitement exacte. Le retrait de l'endoderme commence au début du stade F, et se fait d'avant en arrière, à partir du niveau des vésicules auditives: mais, à ce moment ce feuillet est encore formé d'au moins deux couches cellulaires de chaque còté de la carèno nerveuse. (Planches, fig. 9:3, es).

Les cellules de l'endoderme secondaire sont d'abord arrondics ou polyédriques par pression réciproque, conme les autres éléments embryonnaires. Lorsque se produit le retrait In leuillet, les cellules s'allongent dans le sens vertical et se disposent en deux couches engrenées pour ainsi dire l'une dans l'autre. Ce changement de forme ne s'obserre qu'au dessous de la région moyenne de chaque lame mésodermique, et dans la partic antérieure de l'embryon. Il résulte de cette disposition que, sur une coupe transversile, l'endoderme se montre formé, au milieu et à ses extrémités, d'une couche 


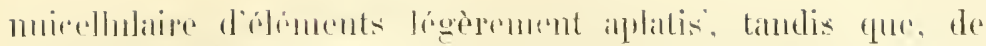
chaque cóté de la carène embryomaire, il présente un épaississmment constitué par deux rangées de cellules allongées.

Ciest au nireau de ces épaississements que se produisent, au state $G$, les plis dinvagination de la partie antérieure du lube digestif, an niveau des futures fentes branchiales. Lal formalliom de cette portion tu canal alimentaire a été bien suvie pill Hoffntann et par Ziegler, qui ont établi qu’il se produisait cur ce point un reploiement de l'endoderme, comme chey les autres Vertébrés, et non un bourgeonnement plein, pénétrant dans le mésoderme, ainsi que le croyait OEllacher. de dois cependant relever une erren d'observation commise par llotfmann, erreur' qui ]'a conduit à assigner all cœur une origine endodermique.

Les deux replis de l'endoderme apparaissent ì une petite distamer de chaque bord lihre de ee fruillet Planches, fig. 100 , ri). D'abord peu marqués et éloignés l'un de l'autre, ils s'accusent de plus en plus en se rapprochant du plan médian, longitudinal, de l'embryon. La portion libre de l'endoderme, située en dehors de chaque pli d"invagination, et formée d'une seule rangée de cellules aplaties, diminue progressivement (Planches, fig. 10I, ri): quand l'iuvagination est terminée et que les bases des deux plis arrivent au contact sur la ligne médiane, de manière à fornuer inférienrement la partie antérieure de l'intestin moyen, tontes les eellules endodermiques sont entrées dans lit constitution des parois de l'intestin. Pour Hoffmann, il reste en dehors des plis d’invagination une rangée de cellules lusiformes, qui ne prennent pas part à la formation du tube digestif, et qui, lors de la frrmeture de co dernier, sont séparées de l'endoderme par les splanchnoplemres.

Malgré les recherches les plus attentives. sur des coupes très minces d'enıbryons bien fixés, je n'ai pu trouver la disposition indiquée par Iloffmann. J'ai toujours vu les cellules endodermiques, en dehors des plis d'invagination, disparaitre progressivement par suite de leur pénétration dans l’intérieur de ces plis. Finalement, il ne reste plus ì la surface du parablaste que la couche profonde de la splanchnopleure. Il résulte de ce fait que, à la fin du stade $\mathrm{II}$, la paroi du sac vitellin est formée dans la région antérieure de l'embryon, par l'ectoderme et le 
mésoderme, et que l'endorderme est complètentent séparé de sil surface. Plus tard, Jendoderne se sépare anssi du parablinte, dans le reste de la longueur de l'embryon, de sorte que la masse vitelline est toute rntière siluée en dehors de lintestin, et comprise entre l'ectoderne, formant la paroi ventrale du sac et les splanchopleures qui forment sa paroi torsal. Lal situation extra-intestinale du sac vitellin avait été lléjì constatéc par Balfour (9), chez les Salmonides. Les anciens auteurs, ron Baer (4) et Lereboullet 112), admuttaient, au contraire, une communication entre le sic vitellin et lintestin; pour le promier, elle arait lieu inmédiatement en arlière du foie, pour le second, entre le foie et lestomac. Hoffmann (89) reconnait aussi, arec Ballour, que le sac ritellin est sans communication avee lüntestin, mais, d’ipres lui, sa paroi dorsale serait furméce par une conche endodermique. Ziegler (200) a reconnu, comme moi, que cette couche appartient au mésoderme et non à l'endoderme.

de ne reviendrai pas ici sur le développenent des fentes brancliales que j’ii donné en déerirant les coupes du stade $\mathrm{H}$. Mais je dois indiquer la manière dont se comporte l'endoderme en avant de la région de ces fentes.

Lendoderme secondaire n'existe que lì où l'endoderme primaire s'est dédoublé en mésoderne et en corde dorsale. Cet endoderme primaire persiste en avant de l'estrémite dr la corde dorsale. Dans cette région il devient très difficile de déterminer les limites du feuillet externe et du l'euillet interne. Au stide F, les dem feuillets sont conlondus sur les cótes de la tète, an niveau du vitellus, ant-dessous des résicules optiques. En arrière de ces résicules, la lision persiste, mais i] existe dr chaque eoté du systeme nerveus une lame mésodermique, freu dérelopée qui śest détachée du leuillel inlérieur: il $y$ a done en ce point un endoderme secontaire, se continuant latiralement plus loin que le nésoderme at se mettant en contact direct arec l'ectoderme. Plus en arrière, les lames mésodermiques débordentl'endoderme, par suite de la coneentration de ec dernier jour former les plis d'invagination. Le monvement de retrait de l'endoderme ne se produit passculement des bords de l'enbryon vers la ligne médiane; il a lieu aussi d'avant en arrière. De plus, la partie céphilique de l'endoderme pri- 
maire rui n’a pas donné naissance à des lames mésodermiques, se transforme entièrement en tissu mésodermique. Dès lors. la partie antérieure de la tête, qui précède les vésicules auditives ne renferme plus que l'ectoderme et ses dérivés, et du tissu mésodermique provenant, soit de la transformation directe in silu rle l'endoderme primaire, soit d'un dédoublement de cet endoderme en lames mésodermiques et en endoderme secondaire, qui s'est retiré s'invaginant de laut en bas et d'avant en arrière, pour constituer, en même temps que les plis d’invagination latéranx, le cul-de-sace antérienr de la cavité digeslive, ent-rle-sic pharyngien.

Le mode de formation de lintestin par rapprochement de deus replis latéranx, se continue un peu en arriere de la région des fentes branchiales, mais dams le trone il se morlifie. La concentration de l'endoderme so fait encore rers la ligne médiane mais sous forme d'un epaississement plein; les deux replis sont très ripprochés el so soudent : ils constituent une masece trapézoïde qui se crense d'une cavité frar écartement des cellules (Planches, fig. 10:3, ri). Dans la région postérieure dre l'embryon, l'endoderme ne donne naissance yu'it un seul pli d'invagination. situé sur la ligne médiane et dont les laues internes s'accolent, laissant entre olles ume fente virtuelle qui sagrandira plus tard pour domer la lumière du canal inteslinal (Planches, fig 106, ri ). Les cellules endodermiques. qui n'entrent pas dans la lormation du pli, se concentrent plus tard sur la ligne médiane, et lorment un pédieule, plein, compris entre les deux splanchnopleures. Ce pédicule, qui rattache l’intestin an vitellus, s'atrophir petit à petit, aux stades ultérieurs a la fermeture du blastoderme, jar résorption de ses éléments. Les splanchnopleures se réunissent au-dessous de l'inlestin; la cloison ainsi formée se résorbe à son tour et lintestin entouré de sil gaine mésorlermique devient libre inférienrement. Ce pliénomène ne se produit qu’ì un stade avancé, surtout dams la partic postérieure de l'enbryon.

Les glandes anneves de l'intestin, telles que le foic, le corps thyroüle etc., ne se développaut qu après la fermeture du blasloderme, après le stade 11 , je ne moecuperai pas de leur origine. 
Tésicule de fiupfer. - La formation la plus préeoce el lit plus intéressante de l’endoderme secondaire est l'organe dont jai décrit le développement an stade E, et que jai appelé la vésicule de Trupffer. La formation et la signification de cette vésicule ont donné lieu am opinions les plus direrses.

Lol'sque Kupfer (104) signala, ('n 1868, l'existence d'unevisicule à la partie postéricure de l'embryon de l'Epinoche et des Gobius mimutus et $G$. niger, il la cousidéra comme représentant l'allintoïde des Ammiotes. En 1879 (104 bis) il décrivit, chez le brocliet, une fente traversant le cordon médullaire et mettant en communication la vésicule avec la surface osterue de l'embryon. Pour lui, il se produit à la partie postérieure de l'ombryon une invagination ectoderulique qui arrive jusquau contact du vitellus, et constitue ainsi une gastrula dont les cellules représentent l'endoderme primaire, l'endoderme secondaire provenant du parablaste. Cet auteur arrive à la mème conchsion, en 1884 (106), à la suite de nouvelles recherches sur la Truite, le Brochut ef l'Eperlan. Chez ce dernier Poisson, il figure, planche 1, figure 1, un canal se rendant de la résicule à la lice dorsale de l'embryon et traversant le bourgeon caudal de bas en haut.

Balfour (9), dans son Traité dembryologie, arlmet que la vésicule de hupfer représinte la partie post-anale de l’intrstin des Plagiostomes. Pour hingsley al Conn (93), qui ont rn cet organe cliez le Ctenolubrus, il consisterait en un espace conpris entre l'endoderme et le vitellus et n’aturait pas de paroi cellulaire propre. Telle est aussi l'opinion d'Agassiz el Whitman. J.-T. Cumninghan (34) admet également que la vésicule de Kimptfer est au-dessous de la face rentrale de lintestin et n'est. qu'une dépression du parablaste: elle scruit en rapport aree le blastopore vilellin par me lente virtuclle siluée entre le parablaste et la face inférieure de l'embryon; elle correspondrail ì la gastrula par invagination des Cyelostomes et des l'agiostomes; de mime que che\% res dernicrs Poissons, sil paroi dorsale scrait formée par l'ectodermu réfléchi, sa panoi reutrale par le parablaste. Seulement, taudis que chez les Plagioslomes. l'extrémilé postérienre de l'mubryon reste sonterée au-dessus du vitelhs, chez les Téléostéchs, elle prpose sur le vitellus et la vé- 
sicule de kuplfer ne représente que la portion anterieure de l’ininstin primordial.

Ton liowalewski (103) décrit la formation de la vésienle de liuprfer de la manière suivante : les cellules endodermiques. provenant du parablaste, après la réflevion de l'ectoderme pour former to mésoderme, se disposent radiairement antour d'une petite excaration dirigée vers la lane enveloppante. Celte eveiration n'est probablement que l'espace que j’ai indiqué entre la lame enveloppante, l'ectoderme et lo parablaste. Cette excavation est, suivant l'autenr. la bouche de la gastrula ou te blastopore; elle s'accroit en avant, de lelle sorte que sa paroi cellulaire, formée inférieurenurnt d'une seulr rangée de cellules en contact avec le parablaste, so continur vor's l'extéricur, sous le bord du germe, en une couche régnlièr. constituée par trois on quatre cellules. Le bord interne de ectte eouche et la cavité de la vésicule, indiquée encore seulement par une fente linéaire, forment une ligne concave qui mubrasso la future extrémité postérieure de la corde dorsale. Collo ligne représuterait le canal neurentérique. La vésicule continue à se déplacer vers la partie antérirure de l'embryon, se crruse d'une carité, et la disposition régulière des cellules du cordon, qui la rattache ì la surface de l'embryon, disparait. Chw le Gobizs, J'ouverture de la gastrula est recouverte par la linne enveloppante. Dans la suite du développement, la vésicule redevient solide par suite de l'invagination sur elle-mème de sa couche inférieure. La cavité de l'intestin post-anal apparait très probablement à la place où existait primitivement celle de la vésicule. La vésicule devenue solide se prolonge en avant sous forme d'un cordon cellulaire axial, que von Kowalewslí regarde comme l'endoderme aux dépens duquel se développerait la corde dorsale.

En somme la manière de voir de von howalewsli se rapproche de celle de Cunningham. Tous deux comparent l'extremité postérieure de l'embryon des Téléostérns à celle de l'enbryon des Plagiostomes; sculement, pour le premier auteur, le plancher de la vésicule est formé par des cellules dérivant du parablaste, pour le second, il est constitué par le parablaste lui-même.

Ziegler (201) se demande pourquoi liowalewslíi ne considère

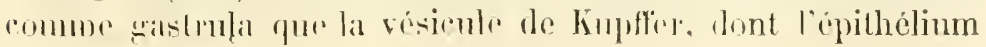


ne représente quine petite partie de tout l'endoderme. Il croit que l'entoderme des Téléstéens est thomologue de celui des Anpluibiens et que la iésicule de himpfer ne représente qu'um minime partie de la cavitr gastruléenne; la gastrula « es en grande partio drpourve de cavité; sa paroi inléricure est formce par le parablaste et non par des cellules difforencieses. Ziegler se range par conséquent à l'opinion do Cunninglamm.

Lorsque, en 1880, je signalai l'existence de da vésicule de kuptfer chez la Truite (69), me fondant sur l'existence d'me ouvirture situéc à la partie postéricure et dorsale que javais obsorvée aul-dessus to la vésicule, chez la Perche, je considérais cette formation comme l'homologue de lintestin primitif des Cyclostomes et des Amphibiens. Depuis cette époque jai pus suive le dévoloppement el J'érolution ultérieure de la résicule et modifier ma manière de voir.

Chez la Truite, Ia résicule de kimpfer naplarait quan stakle E, par conséquent, aprés la formation de la coprle dorsale. des lames mésodemiques et la différenciation de l'endoderne secondaire. Ln certain nombre de cellules de ce feuillet, situées en avant du bourgeon caudal, grossissent, sallongent dans Io sens vertical, et se multiplient par cytodiérèse : il en résulte me pelite masse collulaime qui fiat saillie an milieu res cellules nodolemiques ou indifferentes, qui sont en arrière de l'extrémite postérieure de la corde dorsale. Cette masse cellulairo se crense d’une cavité, ot domne naissinne à une résicule dont lr plancher reposant sur le parabliste est formé par une couche unique de cellules aplaties, et dont le toit convexe est conslitué par mue rangér de collules eylindriques (Planches, fig. 108, h).

Lat cavité lo la résicule s'augmente; l'organe ainsi développé refoule cu lanut lextrémité de la corde dorsalé, et est directement en conțet avec la parlic inférieure te la carène nerveuse dins sa partie postérieure. Primilivement de forme ovoüde. la vésienle sallonge en avant aux stades suirants et devient piriforme; sa paroi supéricure, lans sa portion postérieure, est alor's mal délinitéc du lissu de la corde dorsale qui, à ce nivean, eât très développée et n'est pas différenciée de l'nnduderme. Plus antérienrement, la cavité de la vésicule derient 
très petite et se continue dans l'épaississement endodermique, aux dépens duquel se développe l'épithélimm intestinal (Planches, fig. 109, $k$ ).

La vésicule de kimffer n'est done que la première apparition de la cavité du tube digestif, avec laquelle elle se confond plus tard; mais cettr portion du tube digestif est très importante ì cause des rapports qu'elle affecte avec le système nerveux et la corde dorsale, rapports que jai déjà indiqués ef qui montrent que cotte région correspond à la région du canal neurentérique des antres Vertébrés.

Chez la 'Truite, je n’ai jamais observé de communication entre la vésicule et l'extéricur, soit par un canal en avant du bourgeon camlil, comme l'admet liupffer, soit par une ligne ef une cordon collulaire, comme le dit liomalewshi; je ne puis admettre, en effet, que les quelques cellules aplaties, qui se trouvent ì la face interne de la lame enveloppante, puissent être regardées comme représentant le canal d'invagination de la vésicule.

Chez la l'erche, dont l'embryon se forme tardivement, lorsque le blastoderme a presque totalement recouvert le vitellus, la vésicule de liupffer n'apparait qu'après la fermture du blastoderme, lorsque tout le bourrelet blastodermique, qui entoure le trou vitellin, s’est sondé au bourgeon caudal. Sur l’embryon vivant, jai vu ì sa partic postérieure, un peu en arrière et au-dessus de la vésicule, un orifice à bord plissés, que j’ai considéré, en 1880, comme étant l'ouverture d’invagination de la vésicule ; mais je n'ai pu m'assurer de la continuité de l'orifice arec la visicule. Ayant retrouré une disposition analogue chez la Truite, jai constaté que l'orifice, qui, ici, est très éloigné de la vésicule, n'est que le dernier vestige du trou vitellin après la fermeture du b]astoderme; il est done très probahle qu'il en est de mème pour la Perche, et que l'orifice de la région postérieure n’a aucune relation avee la vésicule de límpffer.

De ce que je n'ai pas observé de communication entre la vésicule et l'extérieur dans les espèces que j’ai examinées, Truite Epinoche, Perche, Lepadogaster, je ne me crois pas autorisé à conclure que cette communication ne puisse exister chez d'autres Poissons, entre autres chez le Brochet et l'Eperlan, où liupffer dit l'avoir vue. En admettant comme exactes les obser- 
vations de cet auteur, la présence d'un canal à la partie postérieure de l'embryon me semble une raison majeure pour admettre l'homologie de cette région avec celle du canal neurentérique, puisque, dans ce cas le canal met en rapport l'intestin primordial avec le sillon médullaire superficiel.

L'opinion des auteurs que, tels que Kingsley et Conn, Agassiz et Whitman, Cunningham, Ziegler, qui admettent, que la vésicule de Kupffer est formée par une dépression comprise entre la partie postérieure de l'embryon et le parablaste, et en tirent par conséquent des conclusions théoriques, est inadmissible parce qu'elle repose très probablement sur une erreur d'observation. La vésicule est, en effet, fermée inférieurement par une couche de cellules; elle est entièrement comprise dans l'épaisseur de l'endoderme. Mais ce qui a pu induire ces observateurs en erreur, c'est que très souvent il existe au-dessous du plancher de la vésicule, ou dans son voisinage, généralement un peu en arrière, une dépression lémisphérique à la surface du vitellus, dans l'épaisseur du parablaste. Cette cavité renferme presque toujours des éléments particuliers que je décrirai à propos de l'évolution du parablaste; ce fait explique la description donnée par les auteurs qui disent avoir vu la résicule remulie d'éléments cellulaires en voie de destruction.

La première interprétation de la vésicule donnée par Kupffer, qui la considère comme une allantoïde rudimentaire, pent être encore défendue en ne considérant, ainsi que je fais, cet organe que comme la première apparition de la cavité du tube digestif, en relation avec le canal neurentérique. L'allantoïde des Vertébrés supérieurs n'est en effet, d’après les travaux de Dobrynin (36), Gasser (52 bis), Matlias Duval (41), qu'un diverticulum de l'intestin, apparaissant de très bonne heure, en avant du canal neurentérique. Si, clez ces animaux, l'allantö̈de est, au déhut, entourée de mésoderme, cela tient à ce qu'elle ne se forme que lorsque la partie postérieure de l'embryon commence à se soulever au-dessus du blastoderme pour donner naissance au capuchon caudal de l'amnios. Chez les Poissons osseux, l'amnios ne se développe pas, le rudiment de l'allantoïde apparaît plus tôt que chez les Amniotes et n'est constitué que par l'endoderme non encore entouré de mésoderme. Je crois done que 
lis manière de voir de kupffer peut ètre acceptée, et qu'on peut regarder la cavité primordiale de l'intestin, située en avant, du bourgeon caudal, comme représentant une allantoïde rudimentaire.

Je discuterai plus loin, à propos de la ligne primilive, l'opiuion qui consiste à voir dans le vésicule de liupfer une gastrula par invagination.

Tige subnotochordale. - L'endloderme secondaire donne naissance, en outre des productions que nous venons d'examiner, à un organe encore problématique, la tige subnotoclordale ou sous-notochordale. Cet organe, découvert par Gœtte (59) chez les Amphibiens, a été retrouvé par Balfour (8), chez les Plagiostomes et le Lépidostée, par Salensky (163), chez l'Esturgeon, et par Balfour (9) chez les Poissons osseux. Balfour en a bien suivi le développement et a vu qu'il provenait de l'endoderme.

Chez les Plagiostomes, la tige subnotochordale apparaît sous forme d'un bourgeon de la paroi dorsale du tube digestif. Dans l'intérieur de ce bourgeon pénètre un diverticulum de la cavité intestinale. Cette saillie s'isole de l'intestin comme un cordon, et la séparation se fait d'avant en arrière, inversement par conséquent de la séparation de la corde dorsale. Dans la partie postérieure de l'embryon, la tige se différencie de la paroi de l'intestin sous forme d'un cordon cellulaire plein. L'organe se développe dans le tronc, puis dans la tête; il est situé immédiatement au-dessous de la corde dorsale, et s'étend en avant jusqu'aux vésicules auditives un peu en arrière de l'extrémité de la corde.

La tige subnotochordale s'atrophie de bonne heure et disparaît d'avant en arrière.

Chez les Poissons osseux, cet organe ne parait pas avoir attiré l'attention des embryogénistes. OfHlacher l'a cependant figuré assez exactement sur ses coupes, mais il considère le petit groupe de cellules placé entre l'endoderme et la corde dorsale comme la première ébauche de l'aorte. Ziegler (102), dans un travail récent sur l'origine du sang chez chez les Téléostéens, a aussi représenté la tige subnotochordale sur plusieurs de ses figurres et l'a indiquén dans la légemule de ses 
planches, mais sans en parler dans le texte. Iloffmann (89) et Goronowitsch (5y) n'y font aucune allusion.

D'après mes observations, la lige sulbnotochordale se développe, chez la Truite, de la même manière que chez les Plagiostomes. Elle apparait, au stade II, dans la portion moyenne de l'embryon sous forme d'une petite masse de trois ou quatre cellules, qui se détache de la partie supérieure du repli endodermique donnant naissance à l"intestin (Planches, fig. 10:, $t$ ); elle se différencie d'arant en arrière, mais elle est constituée par un cordon plein, ne communiquant jamais avec la cavité virtuelle di l"intestin. Elle ne se développe dans la région céphalique qu'aux stades ultérieurs à la fermeture du blastoderme.

Cet organe rudimentaire, entrevu chez le loulet par Balfour ect Marshall, existe très probablement chez tous les Vertébrés, it une période relativement tardive du développement. Doit-on le considérer comme un organe ancestral, et comme représentant, suivant Eisig (45) le tube qui existe au-dessous de la cavité digestive, chez les Capitellides? Je crois qu il est impossible de se prononecr sur sa signification dans l'état actuel de nos connaissances.

IX. - Le MÉsonerue et SEs nérivés.

C'est aux dépens du mésodlerme, dont nous avons vu l'origine au chapitre précédent, que se forment les protovertèbres, la cavité du corps, le système urogénital, et le système circulatoire, cour, vaisseaux et sang.

La lame mésodermique est primitivement une masse pleine comprise cntre l'ectoderme et l'endoderme : elle conserve cette structure à la partie postérieure de l'embryon, jusquà la fermeture du blastoderme et quelque temps après, dams la région où il nexiste pas encore de protovertibres. Elle change, au contraire, d'aspect dans la région médiane dès que les somites apparaissent.

Protovertèbres. - Les premières protovertèbres commencent à se différenciè à la fin du stade E. La partie proximale de la lame mésorlermique s'épaissit, tandis que la partie distale devient plus mines; la partie supérieure de la portion renflée 


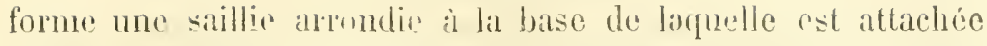
lit partie annincic, au point de jonction de ces deus moities inégales de la lame appirail, sur une coupe transversile, une ligne claire, dirigée de haut en bas et à convexité externe, qui sépare la masse protorertébrale de la masse latérale. En mème temps, nne autre ligne claire se forme dans lépaisseur de la lame latérale et la séprare en deux couches à peu près d’égale épaisseur, la somatopleure d la splamehnopleure. La ligne qui se transforme en fente, par l'écartement des deux couches cellulares, est lit première ébauche de la carvité du corps ou colome; elle ne s'étend pas dims toute la lirgeur de la lame latérale, de sorte que la somatopleure et la splanchnopleure restent unies à leurs deux extrénités. La nuasso protovertébrale est une masse pleine, forméc d'un grand nombre de cellules, dont les périphériques se disposent en une eouehe régulière (Planches, fig. $112, p l$ ).

Lal division métamérique te la lame mésodermique est déjà visible sur des embryons examines par transparence, ou sur des coupes longitudinales, alors qu'elle ne l'est pas encore sur des coupes transversales. Cela tient ì ce que chaque masse protovertébrale s̈isole d'abord de la masse qui la précède, t de celle qui la suit avant de se séparer de la lame latérale, à laquelle elle reste attichée quelque temps. C'est ce qui se voit très bien sur la fig. "\#6(Planches), où les masses protovertébrales sont représentées par des rectangles, limités antérieurement, postérieurement et du còté de l'axe nerveux par une rangée régulière de cellules, tandis que du cóté externe, il n'existe pas encore de limite nette.

Lorsque la masse protovertébrale s'est détachée de la masse latérale, elle appariit sur des compes horizontales ou longitudinales sous forme d'un rectangle neltement circonscrit ì grand axe dirigé de dedlans en dehors dans le premier cas, vertical dins le second (Planches, fig. $111, p t$ ); sur dos coupes transversales la masse présente un aspect variable suivant les régions; elle a en général la forme d'un trapèzè ì angles arrondis, dont le còté supérieur est plus grand que le coite inféricur.

La protovertébre oceupe au début toute la hauteur de l'embryon; elle s'étend de l'ectoderme à l'endoderme. Hais bieniùt 
les cellules de la partie proximale de la lame latérale prolifèrent et donnent naissance à une masse cellulaire, la lame intermédiaire d'OEllacher, qui est située entre la face inférieure et externe de la protovertèbre et la face interne de la lame latérale. Cette masse intermédiaire, s’insinue entre la protovertèbre et l'endoderme ct arrive au contact de la corde dorsale. Elle se sépare alors de la lame latérale et constitue une masse distincte, ovalaire, trapézoïdale ou losangique, sur les coupes transversales, suivint la région où on la considère. En delors de cette masse, netlement circonscrite, se trouvent des cellules irrégulièrement disposées, qui proviennent de la lanıe latérale et pénètrent dans les interstices compris entre la protovertèbre, l'axe nervenx, lí corde dorsale, l'endoderme el la masse intermédiaire; ces cellules qui ne tardent pas à pousser des prolongements et à devenir étoilées, sont l'origine du tissu conjonctif, le mésenchyme. La masse intermédiaire, dont les cellules conservent le caractére embryonnaire et restent arrondies, est la partie qui donnera plus tard naissance aux vilisseaux, aorte et veines, ainsi que l'ont bien établi Wenckebach (196-19y) et Ziegler (201).

Dans la région antérieure de l'embryon, où il ne se forme pas de protovertèbres, la lame mésodermique est, au stade G, étrangléc entre l'ectoderme et le pli d'invagination de l'endoderme; sa partie interne comprise entre le pli endodermique, l'axe nerveux et li corde dorsale, devient une lame céphalique, dontles cellules, dans sa portion supérieure, se disposent en une masse compacte constituant une sorte de fausse protovertèbre, qui donnera plus tard naissance à des faisceaux musculaires, tandis que dans sa portion inférieure, les cellules deriennent étoilées et forment du mésenchyme. La partie située en dehors du pli endodermique, se creuse d'une cavité beaucoup 'plus développée que celle qui existe dans la région du tronc. Cette cavité en continuité avec le cœlome est la cavité péricardique, elle exisie jusqu'au niveau de l'extrémité antérieure de l'intestin et provient de la lame latérale; antérieurement elle se prolonge jusque dans le roisinage de la vésicule oculaire, mais elle a une autre origine : elle résulte d'un écartement des cellules de la partie infẻrieure du mésoderme, qui provient de la transformation de l'endoderme primaire. 
Les deux feuillets de la eavité péricardique, la somatopleure el la splanchnopleure nont pas la même épaisseur dans toute leur étendue: dans leur partie externe, ils sont formés de cellules aplaties, disposées en une seule couche; tandis que leur partie proximale en contact avec l'endoderme est épaisse et comprend plusieurs couches de cellules. Cette région à une grande importanee, e'est d'elle en effet que proviennent les cellules aux dépens desquelles se formera le cœur. (Planches, fig. 101 ct 114.)

Au stade $\mathrm{H}$, la région cardiaque de l'embryon commence à se soulever au-dessus des vitellus, la cavité péricardique devient très grande, sa somatopleure est appliquée contre l'ecdoderme, la splanchnopleure repose en partie sur la parablaste par suite de la soudure des replis endodermiques sur la ligne médiane.

Quelle que soit la région de l'embryon où on la considère, la cavité du ccelome est toujours close, la splanchnopleure et la somatopleure restant confondues à leur partic “listale.

Les deux cavités du colome, situées de chaque côté du corps sont primitivement indépendantes, elles finissent par communiquer entre elles, lorsque l'intestin complètement développé se sépare du vitellus. La communication s'établit d'abord dans la région céphalique en avant du cour.

Le mode de développement que je viens dindiquer pour les protovertèbres et pour le cœlome avait été très exactement suivi par OEHacher (123), rt ma description est à peu près conforme à la sienne, saul quelques points de détails peu importants. Ziegler (200) donne également pour ces organes un processus de formation à pert près identique. Bien différente, au contraire, est la manière de voir de G. K. Hoffimann.

D'après Hoffmann (89) le mode de formation des protovertèbres serait le nême que chez les Plagiostomes. La lame mésodermique se diviserait en deux couches, somatopleure et splanchnopleure dans toute sa largeur. La portion proximale épaissie se différencierait en une protovertèbre dont la cavité se continuerait aver celle qui sépare les deux couches mésodermiques; plus tard toute communication cesserait d'exister entre le colome et la protovertèbre; celle-ci se présenterait alors sous forme d'une vésicule arrondie dont les parois ne 
seraient constituées que par une seule rangée de cellules allongées, disposées en rayonnant autour d'une cavité centrale. De plus, Hoffmann dit ne pas comprendre ce qu'OEllacher entend par masse intermédiaire. Les figures quïl donne des divers stades du développement des protovertìbres différent tellement de celles qui ont été représentées par les autres auteurs, qu'il est difficile de concevoir de quelle manière cet embryologiste a été amené à interpréter ainsi ses coupes: d'autant plus que ses récherches ont porté sur la mème espèce étudiée par OEllacher et par moi, sur la Truite.

Jamais je n’ai vu la cavité du cœlome arriver jusqu’à la protovertèbre, ni celle-ci creusée $d_{\iota}$ une cavité dans les premiers temps de sa formation. Je l'ai toujours trouvée au début constituée par une masse pleine, comprenant sur une coupe plusicurs couches de cellules. Plus tard, à un stade déjà avancé, apparait dans le centre de la protovertèbre, une cavité ou plutôt une fente presque virtuelle par écartement des cellules. Sur des embryons durcis par l'acile chromique, on voit souvent le centre de la protovertèbre occupé par des cellules en voie de destruction, ce qui pourrait faire croire à l'existence d'une grande cavité, mais c'est là un effet du réactif que jai signalé à plusieurs reprises.

Quant à la masse intermédiaire, elle n'existe pas encore au moment de la différenciation des protovertèbres, mais, au stade $\mathrm{H}$ et surtout plus tard, elle est tellement développée qüil est vraiment surprenant que Hoffmann ne l'ait pas observée; il a suivi, en effet, le développement du foic qui n'apparait qu'après la fermeture du blastoderme, à une époque, oì par conséquent, la masse intermédiaire a une grande importance.

Canal de Wolff. - Depuis les recherches de Rosenberg (149) tous les auteurs, sont d'aceord pour faire provenir le canal de Wolff, chez les Téléostéens, de la lame latérale du mésoderme, et en particulier de la somatopleure. Je rappellerai seulement ici que les deux camaur de Wolff apparaissent senlement au stade II, dans la région moyenne de l'embryon; chacun d'eux se présente sous forme d'un repli externe de la partie proximale de la somatopleure à son point d'union avec le splanchnopleure. (Planches, fig. 106, vo). Plus tard, le pli 
s'isole et se transforme en canal qui vient se placer sous la milsse intermédiaire. La différenciation dı canal de Wolli malrche d'avant en arrière, et le canal reste en communication aree le cœlome à son extrémité antéricure; jajouterai que l'origine mésodermique du camal de Wolff est un point imliscutable chez les Téléostéens, ce qui est absolument contraire à l'opinion sontenue récemment par Rabl (130) Flemming (49) Van Wijlie (199) beard (15) qui font provenir le canal chez les Mammifères et les Plagiostones, d'une invagination ectodermique.

Ziegler (201) a vu, à un stade précoce du développement, chez la Truite, de grosses cellules contenues dans l'épaisseur de la lame pariétale, en face des canaux de Wollf; il les considère comme des ovules primordiaux de l'épithélium germinatif. J'ai observé aussi plusicurs fois ces éléments sur de jeunes embryons, même dès le stade $G$, mais ils noceupaient pas unt position constante. J'en ai retrouvé de semblables dans d'antres régions du corps, dans l'endoderme par exenple, mais le plus sowvent dans le mésoderine. Il mest liffieile de me prononerr sur la valeur morphologique de ces cellules, n’ayant pul les suivre jusqu'ì la formation des plis geminatifs qui n’aplarilssent qu'au moment de l'éclosion. Je croirais phrtòt que ce ne sont que des cellules hypertrophiées qui vont entier en cytodiérèse.

Cour. - Les anciens embryogénistes, entre autres Vogt et Lereboullet, ont bien lécrit les premières phases du développement du ccur, visibles extérieurement, mais leurs observations ne nous renseignent pas sur l'origine de eet organe. C'est seulement sur des compes qu'on peut saisir la première ébauche du cœur el déterminer aux dépens de quel leuillet il se forme, et Ziegler a raison de ne pas accorder une grande autorité aux auteurs qui se sont contentés d'étudier l'embryon vivant, par transparence.

OEllacher (123), qui a le premier suivi l'origine du cœur sur des coupes, a bien vu du premier coup de quelle manière se développe cet organe chez les Téléostéens. Ziegler (200) n'a pu que confirmer el préciser sa description, et le résultat de mes observations est absolument semblable à celui de ces'deur auteurs. Seul Hoffmamn est arrivé à des conchusions différentes et 
en désaccord arec nos données actuelles sur le développement du cour chez les autres Vertébrés.

OEllacher et Ziegler font provenir l'endothélium cardiaque de la partic interne des lames latérales mésodermiques, lorsque ses lames vont se rejoindre sur la ligne médiane, au-dessous de l'intestin. Pour Hoffmann (89), au contraire, cet endothélium dériverait d'une couche de cellules endodermiques, qui persiste à la surface du parablaste, après la fermeture de l'intestin antérieur, du pharynx. J'ai déjà discuté crtte manière de voir de Hoffmann, et montré que cette prétendue couche endodermique n'existe pas, et que les cellules qui donnent naissance it l'endothélium du cœur se détachent de la splanchnopleure ou de son point d'union avec la somatopleure; l'opinion de cet auteur me parait done inadmissible.

Ziegler fait remonter la première apparition des rudiments cardiaques au stade G. A ce stade, en effet, dans la région pharyngienne, la partie proximale des lames latérales est épaissie, et s'avance vers la partie médiane, entre le pli d'invagination dle l'endoderme et le parablaste. C'est bien, en effet, cette portion dı mésoderme qui formera l'endothélium, mais le cœur ne commence réellement à apparaître qu'au stade $\mathrm{H}$, quand les lames mésodermiques sont peu éloignées l’une de l'autre.

Suivant OEllacher et Ziegler, la cavité cardiaque est dès le début unique et située sur la ligne médiane de l'embryon. D’après mes observations, le cœur a une double origine; il se produit un tube endothélial à la partie interne de chaque splanchnopleure, et ces tubes se fusionnent ensuite sur la ligne médiane. Ce mode de développement du cœur est le mème que celui qui s'observe chez les Oiseaur et les Mammifères. Kupffer (104) et Balfour (9) avaient signalé la dualité du cœur chez les Téléostéens; mais ce dernier auteur ajoute qu'il se développe avant la fermeture du pharynx. Ce dernier point n'ist pas exact; le pliarynx est déjà fermé quand apparaissent les deux tubes cardiaques, aussi leur duréc est-clle très courte.

Les parois du cœur proviennent de cellules qui se détachent des lames latérales et pénètrent au-dessus et au-dessous de l'endothélium, pour se disposer ensuite en couche continue. Les transformations ultérieures de l'organe, n'ayant lieu qu'après la fermeture du blastoderme, je ne m'en occuperai pas ici. Je 
ferai seulement remarquer en terminant que te coeur el les gros vaisseaux avec lesquels il est plus tard en rapport, ont la même origine; ceux-ei se forment aus dépens des masses intermédiaires, le cour provient de la partic proximale des lames latérales, c'est-à-dire de la partie d'oì dérivent les masses intermédiaires. Cette communauté d'origine démontre, comme le prouve également l’anatomic comparée, que le eour n'est qu’une partie dilatéc du système vasculaire.

\section{X. - Le Parablaste et le sang}

En étudiant la segmentation de l'œul, j’ai fait conmaitre lorigine de la conchre protoplasmique sous-jacente au germe, du parablaste, ainsi que les phénomènes dont cette couche est le siège; j’ai exposé les différentes opinions émises par les auteurs sur le rôle du parablaste pendant la formation des feuillets embryonnaires, et je suis arrivé ì cette conclusion, que cette couche ne prend aucune part à la constitution de l'endoderme. 11 me reste ì dire ce que devient le parablaste durant lo développement de l'embryon.

La couche parablastique, qui existe au-dessous du germe et tout autour de lui ì une certaine distance, s'étend à la surface du vitellus en mêne temps que lr germe. A mesure que sa surface augmente, les noyaux qu'elle renferme se multiplient, mais toujours par division directe. Son épaisseur est très variable; mince dans toute l'étendue de la eavité germinative, le parablaste s'épaissit au niveau de l'embryon et du bourrelet blastodermique. Ses maxima d'épaisseur s'observent généralement au-dessous des régions où le développement est le plus actif. Ses noyaux y sont disposés tout à fait irrégulièrement, tantôt isolés, tantôt réunis par petits groupes de deux, trois ou quatre; ils sont presque tous ì une petite distanee de la surface; on en voit pen dans la profondeur du parablaste. Au moment de la fermeture du blastoderme, le parablaste entoure complètement le globe vitellin : son aspect général n’a pas changé, et il ne subit du reste pas de modifications aux stades ultérieurs du développement.

Outre le rôle que certains embryogénistes font jouer au parablaste dans la constitution de l'endoderme, rôle sul' lequel je n'ai pas à revenir ici, plusieurs auteurs font dériver de cette 
couche protoplasinique les globules sanguins, c'est pour cette raison que jai réservé l'origine de ces éléments pour le présent chapitre.

Baumgærtner (13) en 1830, décrivit les globules sanguins de la Truite comme dérivant d'éléments vitellins modifiés. Yon Baer (4) donna une description peu claire de l'origine lu sang, chez les Cyprins, et le fit provenir d'un blastéme embryonnaire. Hax. Schultze (169) dit avoir vu ehez les Gyprins et la Perche, des splières vitellines se transformer en noyaux, qui plus tard s'entourent d'une membrane et constituent des giobules sanguins. Telle est aussi l'opinion de de Filippi (46). Pour Vogt (191) il n'existe pas de foyer particulier pour la formation de cellules du sang, toute cellule de l'embryon peut se transformer en globule sanguin. Mais, quand les organes sont différeneiés, il se forme à la surface du vitellus une couche hématogène: cette couche "adhùre fortement à la couche épidermoïlale et se compose de grandes cellules transparentes, très serrées, qui renferment pour la plupart un noyau distinet. "Elle ne provient pas du vitellus, et Vogt n̋̈indique pas son origine. Aubert (3), jour le Brochet, admet la manière de voir de Vogt. Lereboullet (111-112) fait renir les globules sanguins de cellules préformatives.

Kupffer (104) a étudié la formation du sang dans le sac vitellin des embryons de Gasterosteus et de Spinachia; il a vu ì la surface du vitellus de petits corpuscules réfringents, semblables à des noyiux, situés au dessous du mésoderme, et qu'il dit se transformer en globules singuins et en cellules pigmentaires. Genselı (54) fait provenir les éléments du sang du parablaste qu'il désigne, avec huptfer, sous le nom d'endoderme secondaire; ses recherches ont porté sur le sac vitellin du Brochet et du Zoarces viviparus. La région dans laquelle apparaissent les premiers globules sanguins n'est recouverte que par l'ectoderne; dans le parablaste sous-jacent se forment de grosses cellules amiboüdes avec un ou plusieurs noyaux : ces cellules se colorent fortement par les réactifs, tandis que la masse granuleuse environnante se colore peu. De ces gros hématoblastes se détachent de petites cellules qui restent pendant quelque temps attachées par un pédicule. Les globules primaires ainsi formés sortent de la couche protoplasmique, dans laquelle ils ont pris 
naissanee, et viemnent se loger dans l'espace compris entre l'ertoderme et le parablastu. Ils ne renferment dans leur intérieur qu'un ou plusieurs corpuscules colorables, mais pis de noyaux nets; les globules primaires se divisent ef se groupent en ilots de globules simguins définitifs, pourvus de noyaux permanents.

Hoffmamn (89) n'hésite pas à dire que les noyaux du parablastr ne prennent aucune part i la formation des globules sanguins, qui dérivent, pour lui, de l'endothélium du cœur. Il fait remarquer que les noyaux parablastiques sont surtout nombreux au dessous du bourgeon caudal, là où, dans les premiers stades du développement, se fait une grande production de cellules. Quand le cour est formé, bat et renferme des globules sanguins, on n'observe aucun changement dans le nombre et la grosseur des noyaux du parablaste, et on ne les voit jamais se transformer en globules sanguins. Pour Hoffinann, Je parablaste joue le röle d'une sorte de sang provisoire; il assimile les principes untritifs du vitellus et les transforme en une nourriture assimilable pour les cellules dı germe et plus tard de l'embryon. Si l'on place, en effet, des œufs dans des conditions défavorables pour leur développenent, dans l'eau stagnante par exemple, ils devienuent malades; on voit alors les noyaux du parablaste être les premiers atteints, subir une légénérescence graisseuse; l'embryon ne peut plus alors se nourrir et ne tarde pas à mourir.

Wenckebach (197) et Ziegler (201) considèrent aussi le pariblaste comme un organe provisoire servant à la nutrition de l'embryon. De mème que Iloffmann ils n'ont jamais vu de globules sanguins se lormer liuns cette couche. Ceux-ci proviennent des cellules mésodermiques qui remplissent primitivement les vaisseaux, veines et aorte, lorsqu'ils se différencient aux dépens de la masse intermédiaire. Quant aux vaisseaux el aux globules sanguins des parois dı sac vitellin, ils proviennent de cellules migratrices du mésoderme, qui vout tapisser des goultières creusées à la surface du vitellus. Ces gouttières, d'abord recouvertes seulement par l'ectoderme, se transforment en vaisseanx. clos, par l'arrangement en couche eontinue des cellules migratrices (Wenckebach).

Lexistence de ess enllule: migrafrices est facilo à observer 
sur l'œuf vivant de l'Épinoche; j’ai pu les voir se détacher du hord de l'embryon et s'avancer à la surface du vitellus. In certain nombre de ess cellules se clargent de granulations noires, et deviennent des cellules pigmentaires, les autres s'allongent, acquièrent des prolongements filiformes et prennent lispect des cellules riso-formatives que le professeur Ranvier a étudiées dans l'épiploon du Lapin. Je n'ai pas suivi, d'une façon assez continue l'évolution de ces cellules, pour lire de quelle manière elles s'agencent entre elles pour former des raisseaux. Cette étude est, du reste, en dehors du cadre de mon sujet. Je ne me suis oecupé que de l'origine des premiers globules sanguins intra-embryonnaires, de ceux qui, au stade $\mathrm{H}$, existent déjà dans la cavité du cœur, et je n’ai pu, à ce proint de vue, que confirmer les obserrations de mes prédècesseurs, à savoir que ces éléments sont des cellules dẻtachées de l'endothélium.

Jo me suis surtout attaché ì rechercher le sort des noyaux du parablaste, et à vérifier les recherches de Gensch. Il n'est pas tout a fait exact de dire, aver Hoffmann, que les noyaux dı parablaste ne suhissent ancun changement durant le développement de l'embryon; cette assertion est vraie pour la majorité d'entre eus, mais un certain nombre de ces noyaux sont le siège de transformations intéressantes, observées par Gensch, et mal interprétées par cet auteur.

Von Kowalewski (131), Wenckebach (197) et Zirgler (201) ont signalé la dégénérescence des noyaux du parablaste. Ce dernier auteur représente sur la figure 9 de la planche XXXVI, la fragmentation de ces noyaux; de mon côté jai pu suivre les transformations des élénents parablastiques et leurs destinées ultérieures.

Dès que les noyau du parablaste ont cessé de se multiplier par division indirecte, ce qui a lieu de bonne heure, avant la fin la segmentation, ceux qui ne sont pas devenus le centre de formation de cellules renant s'ajouter au germe, prennent un aspect caractéristique qui a frappé tous les observateurs. Ils deviennent volumineux, à contours irréguliers. presque toujoutrs allongés parallèlement à la surface du parablaste: leur grand diamètre mesure de $0^{\mathrm{mm}}, 02$ ì $0^{\mathrm{mm}}, 03$. Leur intérieur renferme un réscau ì larges mailles, se colnrant fortrment par le 
carmin, l'hématoxyline et les couleurs d'aniline; on y voil alussi souvent un ou plusieurs corpuscules également trìs colorés, silués sur le trajet du réseau, et qui peuvent être regardés comme des nucléoles.

Pendant le développement de l'embryon, à peu près à partir du stade F, parmi les nombreux noyaux accumulés sous l'embryon, principalement sous le bourgeon caudal et sous la tête, on en voit un certain nombre dont le réseau chromatique devient moins net, se résorbe par place, et cesse de se colorer d'une manière aussi intense sous l'influence dles réactifs. Ces noyaux ne tardent pas à sc fragmenter, tantôt assez régulic rement par bipartition, tantòt au contraire, par une sorte de gemmation; chaque petit fragment, qui a à peu près le volume d'une cellule embryonnaire, est globuleux; il est entouré par une membrane mince, et renterme un protoplasma granuleux, creusé d'une ou deux grandes vacuoles; il possède aussi un, deux, rarement trois grains réfringents de chromatine, prenant une teinte foncée avec le carmin ou l'hématoxyline, tandis que le reste du globule est tout à fait incolore. Ces globules ressemblent beancoup aux cellules dégénérées que Flemming (48) a décrites dans la granulosa des follicules de Graaf en voie de régression. (Planches, fig. 116, gp.)

Les globules parablastiques, après s'être sẻparés des noyaux qui leur ont donné naissance, se rapprochent de la surface du parablaste, et finissent par en ètre expulsés. Je n'ai pu suivre, sur le vivant la formation et les migrations des globules, aussi je ne puis dire si ces éléments sont doués de mouvements; mais il me parait difficile d'admettre qu'ils ne se déplacent pas comme les cellules migratrices. On retrouve, en effet, ces globules dans les feuillets embryonnaires, et quelquefois très loin de leur lieu d'origine. On les reconnaît facilement au milieu des cellules, grâce à leurs grains de chromatine. Si l'on examine une coupe, en la noyant dans la lumière, à l'aide de l'éclairage d'Abbe, comme cela se fait pour la recherehe des microbes, on voit les figures cytodiérétiques et les grains colorés des globules parablastiques trancher par leur vive coloration sur le fond de la préparation.

Souvent, au-dessus de la région où se sont formés des globules parablastiques, il se produit une dépression à la surface 
du parabliste, atu-dessous de la face inféricure de l'embryon; les globules s'accumulent alors, quelquefois en grand nombre, dans ces cavités. Celles-ci pariissent se former de préférence en certains points détemines de l'embryou. C'est ainsi qu'on trouve souvent une grande dépression dans le roisinage de la vésicule de Kupffer (Planches, fig. 109, gp); et j’ai indiqué que c'est probablement cette cavité qui a fait croire à Cunningham que la vésicule était comprise entre l'embryon et le parablaste. On en observe fréquemment une autre au-dessous do l'ébauche cardiaque. Ziegler a signalé dans cette région la présence de fragments de noyaux du parablaste, et leur ressemblance arec les cellules migratrices qui donnent naissance au cœur et aux globules singuins; il fait justement remarquer qu'il est très difficile de distinguer ces deux sortes d'éléments. Les cellules migratrices mésoderniques se reconnaissent cependant à leur noyau bien conformé, tandis que les globules parablastiques n'ont que des corjuscules réfringents. C'est évidemment la similitude de ces deux sortes d'éléments qui a induit Gensch en erreur. Cet obscrvateur a bien vu la formation des globules aux dépens des noyaux du parablaste, et leur migration au-dessous de l'ectoderme dans la paroi du sac vitellin, mais il n'i pas su les distinguer des vraies cellules sanguines d'origine mésodermique.

Les globules parablastiques qui pénètrent dans l'embryon peurent se retrouver dans différents organes; dans les lames mésodermiques (Planches, fig. 116), l'endoderme et mème dans l'axe nerveux; jen ai trouvé plusieurs fois, au stade H, dans la cavité cérébrale (Planches, fig. 103, gp). Ils ne tardent pas à ètre résorbés, et ne jonent ancun rôle dans la formation des organes.

Je me range done complètement a lavis de Hoffmann, et je considèr le parablaste, Iorsquł'il a cessé de fournir des éléments cellulailes au germe, comme un organe de nutrition de l'embryon. Non seulement il assimile les éléments nutritifs du ritellus pour les transmettre à l'embryon probablement sous une forme liquide. une sorte de sérum s̈infiltrant au milieu des cellules embryonnaires, mais il fournit aussi ì cet embryon. des éléments figurés provenant de la transformation des noyaux, et servant à la nutrition des tissus. Il est ì présumer 
que les globules parablastiques ne sont pas formés exclusivement par les noyaux, mais que le protoplasma ambiant entre aussi, pour une certaine part, dans leur constitution; à l'appui the cette hypothèse, il est permis, je crois, d'invoquer la migration des globules. Le déplacement de ces éléments s'explique, en effet, sils renferment une certaine quantité de protoplasma ; il wt au contraire difficile ì conceroir sils ne sont constitués que par de la nucléine.

\section{Xl. - Accholssemext he l.enbrios.}

Le mode daccroissement de l'embryon est m des problèmes les moins avancés de l'embryogénie générale. Les recherehes entreprises pour élncider cette importante question sont peu nombreuses et peu précises; aussi la plupart des auteurs se bornent à émettre des hypothèses à ce sujet. Je ne m'occuperai ici que de celles qui ont rapport au développenent des Poissons.

Chez les Vertébrés amniotes, l'embryon est placé au centre du hlastoderme, et celui-ci s'étend sur le vitellus (dans les oufs méroblastiques) d'une manière uniforme, par toute sa périphérie, de telle sorte que l'embryon denteure en place it l'un des pôles de l'œuf, tandis que la fermeture du blastoderme a lieu au pôle opposé.

Chez les autres Vertébrés, l'embryon orcupe au contraire une position excentrique et se développe sur le bord du blastoderme, celui-ci entoure le vitellus et sa lermeture se fait immédiatement en arrière de l'embryon.

hupffer (104), qui s'est posé l'un des premiers la question de savoir comment se produit cette extension, admet qu'elle se fait comme chez les Vertébrés supérieurs, d'une manière égale et escentrique; le centre du blastoderme reste five au pòle germinatif, le bourrelet blastodermique descend progressivement sur le vitellus, parallèlement à lui-mème, suivant des cercles parallèles à l'équateur de l'œuf. L'embryon qui est en comnexion avec le bourrelet, laccompagne dans son mouvement de descente, tout en s'itccroissant en longueur. La fermeture du blastoderme a lieu en un point du vitellus diamètralement opposé à celui où se trouvait le germe (fig. 27). 
L'embryon s'accroit par intussusception dans toute sa longueur.

En tout cas linpffer n'exclut pas la possibilité d'une déviation de ce processus un peu avant la fermeture du blastoderme, et Ziegler (200) a noté, en effet, que chez le Rhodeus amarus le trou vitellin est situé $u$ peu asynétriquement sur le côté In diamètre vertical. Goronowitsch (5y) ildmet aussi ce mode de formation pour les Salmonides.

Pour OEllacher (123), liı partic épaissic du bourrelet blastodermique, qui donne naissance à l'embryon, reste en place sur un point fixe du vitellus, tandis que le reste du blastoderme s'étend sur la sphère vitelline. Le bourgeon caudal de l'embryon ne change pas de place; la partie céphalique s'accroit ct suit le blastoderme dans "son mouvement dextension (fig. 2:).

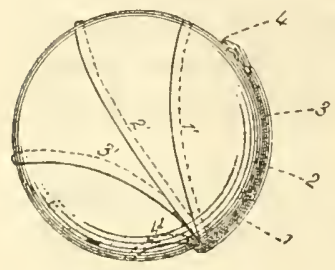

25

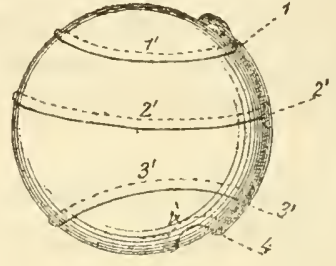

26

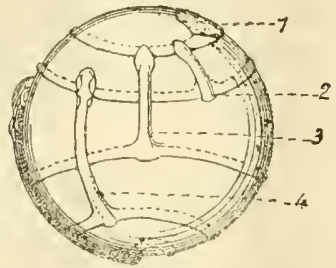

9T

Schémas de l'extension dn hlastoderme à la surface du vilellus de l'enf des Salmonides. -

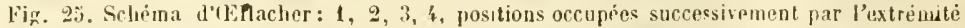
céphalique de l'embyron ; 1', 2 ', 3', ', , positions correspondantes du bourrelet blastodermique. - Fig. 26. Schéma de His : 1, 2, 3, 4, positions occupées successlvement par l'extrémité caudale de l'embryon; 1', 2', 3', 4', positions correspondantes du bourrelet blastodermique. - Fig. 27. Schèma de Kunffer : 1, 2, 3, 4, positions oceupées successivement par l'embryon.

His (85) admet, an contraire, que l'extrémité céphalique reste fixée sur le vitellus pendant l'estension du blastoderme. L'embryon s'allonge aux dépens de denx formations symétriques du bord épaissi du blastoderme. His (84) a primitivement émis cette hypothèse pour les Plagiostomes et l'a étendue ensuite aux Poissons osseur. Chez ces derniers, le bourrelet blastodermique forme une anse, dont les deux borls se rapprochent pour constituer la gouttière mérlullaire; le sommet de l'anse est l'extrémité céphalique; l'extrémité diamétralement opposée du bourrelet est l'extrémité caudale. Le hourrelet blastodermique tout entier se trouve ainsi amené le long de la ligne axiale de l'embryon ponr constituer le corps de ce dernier (fig. 26). Chez les Plagiostomes, l'embryon se formerait de la 
même manière, mais il n'y at qu'une partice du bourperel blastodernique qui frend part ì la ronstitulion de l'embryon, ear celui-ci est complétement formé arant la fermeture du blastodernic.

La théorie de Ilis, oll théorie de lis eonerescence, a été atoptée par Rauber (139) qui a apporté en sa faveur des arguments tirés de la tératologie.

Rauber rappelle que Lereboullet (113) a observé certains wuls de Brochet dans lesquels l'embryon avait une seule tête ot une queue, mais dont le corps était séparé longitudinalement en deux moitiés par un espace blastodermique, an milieu dnquel se tronvait le tron vitellin. Rauber a également observé des germes monstrueux de Truite dans lesquels, il y arait déhiscence de l'anse embryonnaire; le corps de l'embryon ćtait séparé en deux parties qui se continuaient avec le bourrelet blastodermique et laissaient entre elles un espace vitellin, non recouvert par le blastoderme. Cumningham (35) s'est rangé aussi à la théorie de His.

Au premier abord, la théorie de la concrescence de His et Rauber est très séduisante; elle rend bien compte de la position excentrique de l'embryon, toujours en rapport avec le bourrelet blastodermique, et de la fermeture du blastoderme en arrière de l'embryon. Liadjonction à l'embryon du reste du bourrelet blastodermique, lors de cette fermeture, devient très compréhensible puisquelle n'est que la continuation du proeessus de la formation de l'embryou. Cette théoric est copendant contraire ì certains laits d'observation et a soulevé des critiques sérieuses de la part de plusieurs auteurs, entre autres de la jart de Balfour.

Balfour (9) tire les principanx de ses atguments du développement des Plagiostomes; je n'en citerai que quẻlques-uns. Il fait remarquer que la gouttière médullaire se ferme en arant phus tôt qu'en arrière, et que cette l'ormelure commence lorsque l'embryon est encore très courl et arant que l'extrémité postérieure ait commencé à faire saillie an-dessus du vitellus. "Après que le canal médullaire est fermé êt 'n continuité "In arrière arec le tube digestif par le canal neurentérique, il est évidemment impossible quaueun accroisscment de longuem ait lieu par conerescence. Si par conséquent l'opinion de His at do 
Rauber est acceptée, il faudra soutenir qu'une faible partie du corps seulement se forme par concrescence, tandis que la plus grande partie de la région s’accroît par intussusception. 》 "Chez l'Amphiorus, dit plus loin Balfour, le blastopore est d'abord situé exactement à l'extrémité postérieure du corps, quoique plus tard il passe au côté dorsal, il se ferme presque avant l'apparition de la gouttière médullaire ou des somites mésoblastiques, et les replis médullaires n’ont rien à voir avec sés lèvres, si ce n’est qu’ils sont en continuité avec clles, en arrière exactement comme chez les Elasmobranches.

"Le vitellus nutritif, chez les Vertébrés, est situé à la face ventrale du corps et est enveloppé par le blastoderme, de sorte que, chez tous les Vertébrés à gros vitellus, les parois ventrales du corps sont évidemment complétées par la fermeture des lèvres du blastopore sur le côté ventral. Si His et Rauber ont raison, les parois dorsales sont aussi complétées par la fermeture du blastopore, de sorte que toute la paroi dorsale, aussi bien que la paroi ventrale de l'embryon, doit être formée par la concrescence des lèvres du blastopore, ce qui est évidemment une réfutation par l'absurde de toute la théorie.» (p. 287 et 288.)

On peut amssi opposer à la théorie de la concrescence des faits qui me paraissent lui ètre absolument contraires.

His, arec une patience admirable, s'est livré à de nombreuses mesures micrométriques et à des calculs pour déterminer la surface du blastoderme et le volume de l'embryon aux différents stades du développement. Il a mesuré l'épaisseur de l'ectoderme et de la couche inférieure du blastoderme, le volume des cellules de ces deux couches, et enfin le volume de l'enbryon et du blastoderme extraembryonnaire sur des blastodermes mesurant $4^{\mathrm{mm}}, 7,2^{\mathrm{mm}}, 2,2^{\mathrm{mm}} 7$ et $3^{\mathrm{mm}}, 2$ de diamètre. Ces embryons correspondaient à peu près aux starles A, B, C et D de la Truite, et il les désigne du reste par ces mêmes lettres.

te ne puis rapporter ici toutes les mesures de His; je me contenterai de donner les conclusions auxquelles il a été amené, conclusions qu'il a résumées dans une note publiée dans Internationale Fischerei. Ausstellung :u Berlin, 1880 (Schweiz.)

Le volume d’un embryon de Poisson, immédiatement après l'extension complète du blastoderme sur le vitellus, mesure environ 0,4 millimètre cube, celui de la paroi du sac vi- 
tellin anviron 0.1 millimille "ube; les deux rolumes réunis représentent donc environ $0,5 \%$ millimètre cube. C'est un volume égal que possède déjà le germe avant le commencement de la formation de l'embryon, immédiatement après la fin de la segmentation, La transformation totale du germe en embryon et en paroi du sac vitellin a lieu sans que le volume total éprouve une augmentation perceptible. Dans le germe segmenté il y a les matériaux destinés à la constitution du corps, et il suffit de la transformation de ces matériaux pour donner naissance à l'embryon et à ses membranes. His compare re processus ì ce qui se passe lorsqu'un artiste inodèle une sculpture avec une masse informe de terre glaise, sans prendre une nouvelle quantité de substance. L. volume des matériaux de formation ne reste constant que pendant la période de formation de l'embryon; pendant la segmentation, le volume du germe a à peu près doublé, et à partir de la période d'accroissement, la masse de l'embryon augmente naturellement d'une façon continuelle.

Je n’ai pas cherché à contrôler les mesures micrométriques de His, parce qu'elles demandent beaucoup de temps et beaucoup trop de peine pour le résultat forcément approximatif quelles peuvent dommer. Je les admets cependant comme exactes, et je valis chercher à démontrer qu'elles ne prouvent rien au sujet de la formation de l'embryon par concrescence.

His a donné la mesure des cellules embryonnaires jusqu'au stade $\mathrm{D}$; il a trouvé que le volume moyen de ces éléments était :

\begin{tabular}{|c|c|c|c|c|}
\hline \multirow{3}{*}{ Stade } & & \multirow{3}{*}{$\begin{array}{l}\text { Ectoderme. } \\
180^{4} 7 \mu \text { cubes. } \\
5040\end{array}$} & & \multirow{2}{*}{$\begin{array}{l}\text { Couche inférieure. } \\
12120 u \text { cubes. }\end{array}$} \\
\hline & A. . . & & - . & \\
\hline & li. . . & & - & 7238 \\
\hline & C.. . & 3482 & $\ldots$ & 4849 \\
\hline & 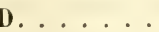 & 1726 & . . & 2144 \\
\hline
\end{tabular}

En rapportant a 100 le volume les cellules au slade $\mathbf{A}$, on trouve :

Stade A

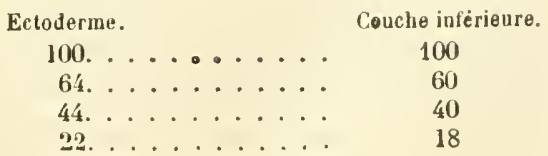

Il résulte de ce tableau que les cellules diminuent de volume du stade $\mathrm{A}$ au stade $\mathrm{D}$ et qu'à ce dernier stade elles sont environ 
quatre fois plus petites quà la fin de la segmentation. Le liait n’a rien qui doive surprendre, étant donnée la multiplieation des cellules embryonnaires, multiplication qui se traduit sur les coupes prar la présence d'un grand nombre de figures cytodiérétiques. Il est évident que, puisque le volume total de la masse embryonnaire ne change pas et que le volumo des éléments diminue, ceux-ci doivent augmenter de nombre.

Au stade D, le blastoderme ne recouvre encore que la moitié environ du vitellus, la partie céphalique de l'embryon est it peine ébauchée. His dit qu'au moment de la fermeture du blastoderme le volume total des éléments cellulaires n'a pas sensiblement augmenté, mais il ne donne pas les dimensions de ces éléments. Jusqu'à ce que l'extension du blastoderme ait atteint l'équateur de l'œuf. la surface du bourrelet marginal doit augInenter; elle diminue au contraire lorsque le bourrelet a dépassé l'équateur.

His a constaté que le bourrelet est formé au stade B par 10 ou 12 rangées de cellules, tandis qu'au stade D il n'en renferme plus que 4 à 6 . L'épaisseur va encore en diminuant aux stades suivants; au stade F, je n'ai plus trouvé que 3 ou 4 rangées de cellules dans le bourrelet.

Je n'ai pas calculé le volume des cellules embryonnaires aux différnts stades, je me suis contenté de prendre leur diamètre moyen. J'ai constaté, comme His, que les cellules diminuaient de volume, après la fin de la segmentation. pendant les premiers stades embryonnaires, mais qu’à partir des stades I) et E leur diamètre ne varie plus. Du reste, ì cette époque, les mesures deviennent très difficiles. Dès que se différencient les principanx organes de l'embryon, les résultats que peuvent donner les mesures micrométriques des cellules sont tout it fait illusoires. Les dispositions que prennent les cellules dans les organes altèrent complètement leur forme primitive plus ou moins arrondie ou polyédrique; les cellules allongées de l'axe nerveux de l'ectoderme, de la corde dorsale, ne sont plus comparables aux cellules non encore différenciées des premiers stades de l'embryon. Cependant en prenant le diamètre des cellules cetodermiques au stade D et des cellules hópatiques d'un joune alevin récemment éclos, cellules qui représentent le volunte moyen des éléments divers que constituent le corps, 
jai trouvé comme mesure moyenne el constante $1 \%$ u. Les cellules cessent done de diminuer de volume arant la formation complète de l'embryon et la fermeture du blastoderme.

Tant qu'il n'y a pas d'organes différenciés dans l'ébauche embryonnaire il est difficile de trouver des points de repère et de voir si ees points chingent de position par rapport au bourrelet blastodermique. Cette observation est au contraire très facile dès que la corde dorsale el la vésicule de Kupffer

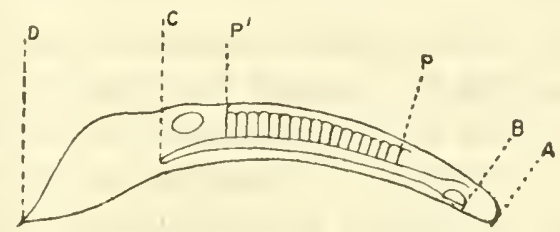

Fig. 28. - Coupe longitudinale d'un embryon de Truite : A, extrémité postérieure du bourreon caudal ; B, extrémité postérieure de la vẻsicule do Kupfier; C, extrémité antérieure de la corde dorsale; $\mathbf{D}$, extrémité antérieure de l'embryon; P, dernière protovartébre; P', premièro protovertèbre.

deviennent nettement visibles soit sur des coupes transversales, soil sur des coupes longitudinales. A partir du stade E, j’ai pu mesurer sur des coupes longitudinales de différents embryons la distance AC qui sépare l'extrémité antérieure de lat corde dorsale de l'extrémité postérieure de l'embryon et celle AB, qui sépare la paroi postérieure interne de la vésicule de Kupffer de cette mème extrémité postérieure. J'ai mesuré également la distance CD qui sépare l'extrémité antérieure de la tête de l'embryon, et la distance qui sépare cette dernièro extrémité de la vésicule auditive (fig. 28).

Ces mesures montrent que, tandis que la longueur totale de

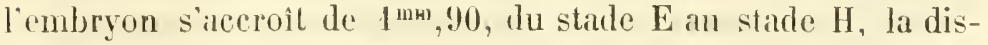
tance qui sépare la vésicule de Kupffer de la partic postéricure du bourgeon caudal n'augmente que de $0^{\mathrm{mm}}, 0.3 \%$. Cette partie postérieure de l'embryon n’a done qu'une croissance insignifiante par rapport à celle du reste du corps. Ryder (154), che\% le Belone longirostris figure aussi la vésicule de Kupffer à une distance à peu près constinte du bord du blastoderme.

Lal comparaison des valeurs de la distance CD, qui mesure la longueur de la tête, montre également que la!̣partie de l'embryon comprise entre la terminaison antérieure de la corde 
dorsale et l'extrémité céphalique n'augmente pas sensiblement pendant l'extension du blastoderme sur le vitellus. Chez un embryon assez avancé, quelques jour's après la fermeture du blastoderme, j’ai trouvé que la distance CD était encore de $0^{\mathrm{mm}}, 76$ tandis qu'elle était de $0^{\mathrm{mm}}, 60$ au stade E.

C'est donc dans la région BC, comprise entre la partie postérieure de la vésicule de hupffer et l'extrémité antérieure de la corde dorsale que se fait le principal allongement de l'embryon.

Le nombre des protovertẻbres augmente assez rapideuent ainsi que je l'ai déjà dit, pendant l'extension du blastoderme : au stade $\mathrm{E}$, il y en a 3 à 4 de chaque côté de l'axe nerveur ; à la fermeture du blastoderme, l'embryon en possède environ 24 paires. Il est très difficile de déterminer la région dans laquelle se forment de nouvelles protovertebres.

Fol (51) a cherché expérimentalement à trouver le mode d'apparition des protovertèbres dans l'embryon de Poulet; eı marquant au thermocautère, sur les côtés de l'aire enbrỵonnaire, le point où se trouvent les première protovertèbres, il a constaté que ces protovertèbres sont celles du cou ou nême de la région céphalique. Fol admet que l'embryon des Vertébrés commence par n'être pour ainsi dire qu'une tête derrière laquelle apparait de proche en proche le reste du corps. L'observation de Fol confirme ce qui est généralement admis par la plupart des auteurs à savoir que les protovertèbres se développent d'avant en arrière.

En est-il de mêne chez les Poissons osseux? Chez un embryon de Truite du stade $\mathbf{E}$ ayant trois paires de protovertèbres, CP', qui mesure la distance de l'extrémité céphalique de l'embryon à la première protovertèbre antérieure, est d'environ $1 \mathrm{~mm}$. Au stade F, ehez un embryon à six paires de protovertèbres, CP' est encore de $1^{\mathrm{mm}}$. Au stade $\mathrm{H}$, chez un embryon à vingt-deux paires de protovertèbres CP, est de $1,1^{\mathrm{mm}}$. Ces mesures prouvent que la première protovertẻbre antérieure reste à une distance à peu près constante de l'extrémité antérieure de l'enbryon; il me semble donc logique d'admettre qu'il ne se forme pas de nouvelle protovertèbre en avant de la première des trois qui existent au stade E. Cette première protovertèbre antérieure se trouve, en effet, aux stades suivants, à quelque distance en 
arrière de la vésicule auditive ef immédiatement en arrière de l'extrémité pustérieure de l'intestin antérieur. La segmentation transversale des plaques mésodermiques en protovertèbres se lait donc chez les T'éléostéens, de même que chez le Poulet, d'avant en arrière.

Si d'un autre côté on mesure la distance AP qui sépare la dernière protovertèbre de l'extrémité postérieure de l'embryon, on trouve qu'elle est en moyenne de $0 \mathrm{~mm}, 65$ depuis l'apparition des protovertèbres, c'est-ì-dire depuis le stale E jusqu’à la fermeture du blastoderme et même quelque temps après. La dernière protovertèbre reste done pendant les premiers stades du développement de l'embryon ì une distance constante de l'extrémité postérieure du corps, comme la première protovertèbre antéricure reste à une distance constante de l'extrémité antérieure de l'embryon. Puisque ni l'extrémité antérieure ni l'extrémité postérieure de l'embryon ne s'accroissent, on devrait en conclure que la partie moyenne subit seule de l'allongement. Sïl en est ainsi, les nouvelles protovertèbres ne peuvent provenir que d'une division des protovertèbres préexistantes, car celles-ci sont exactement contiguës. Or, on ne voit jamais une protovertèbre se diviser pour donner naissance à deux antres. Le diamètre des protovertèbres diminue du stade E au stade H, mais leur hauteur augmente; il y a simplement déplacement des éléments qui les constituent. Il est douc impossible d'admettre que de nouvelles protovertèbres prennent naissance entre la première et la dernière. On se trouve donc amené par le raisonnement à déclarer que l'embryon ne s'accroît ni par sa partie céphalique, ni par sa partie moyenne, ni par sa partie postéricure, ce qui est évidemment absurde. Le raisonnement pêche donc par sa base.

La valeur constante de la distance Al', qui sépare la dernière protovertẻbre de l'extrémité caudale de l'embryon, ne prouve pas, en effet, que cette partie de l'embryon ne s'accroisse pas. Tandis que du côté de la tête il y a des organes nettement différenciés qui servent le point de repère et permettent de constater que la première protovertèbre conserve les mêmes rapports avec ces organes; à la partie postérieure de l'embryon il existe, entre la dernière protovertèbre et la vésicule de Kupffer, un espace d'environ $0^{\mathrm{mm}}, 45$ dans lequel, à part l'axe 
nerveux et la corde dorsale qui ont la même structure que dans le reste de l'embryon, on ne trouve que les lames mésodermiques non différenciées. Il peut done se produire, entre la vésicule $B$ et la dernière protorertèbre P', un allongenent qui passera inaperç s'il se torme en mêrre temps une protovertèbre, et si l’épaisseur de cette nouvelle protovertèbre correspond précisément à l'acceroissement de l'espace BC.

Cette lypothèse, la scule qui me paraisse acceptable, I'est pas me simple vue de l'esprit, elle repose sur un fait d'observation. Lorsqu'on examine des coupes longitudinales d'un embryon pourvu de protovertèbres, on eonstate que la partie antérieure de la lame mésodermique qui fait suite aux protovertèbres présente un léger renflement d'une épaisseur égale à celle des protovertèbres, et dinns lequel les cellules commencent à se disposer radiairement autour d'un centre; le renflement est souvent séparé virtuellement du reste de la lame mésodermique par une ligne à peine visible : il ne peut y avoir de doute qu'on ait sous les yeux une protovertẻbre en voie de formation.

L'enhloyon des Téléostéens s'aceroit done jusqu'ì la fermeture du blastoderme par la partie qui est comprise entre la résicule de Kupffer et les protovertìbres, de noureaux somites se formant constamment à la partie antérieure de cette région au fur et ì mesure qu'elle s'accroît; ce fait me semble nettement étahli par les mesures que j’ai rapportées plus haut. Voyons maintenant si cette donnée est compatible avec lat théorie de His.

Si la théurie de la conterescence de l'embryon est vraie, cette conerescence ne peut se produire quéen avant de la résicule de Kimpfler. si, en eflet, la réunion des deux moitiés du bourrelet blastodernique avait lien en arrière de cette vésieule, celle-ci devrait s'éloigner de plus en plus de l'estrémité candale, dans la suite du développement embryonnaire, ce qui est contraire à l'observation; admettons done que la réunion des deux moiliés du bourrolet se fait en avant de la vésicule et que l'extrémité postérieure de l'embryon reste enchâssée dans l'anse formée par le bord externe dú bourrelet blastodermique, il faut exuminer comment peut se laire ectle rémnion.

Le bourrelet est lormé comme on sait de deux conches, l'une 
supérjoure ectodermique, l'autre inférieure provenant de la réflexion de la première et qui représente l'endodermé primaire 10 l'enbryon. Lat rémnion des deux moitiés du bourrelet peut se faire de deux manières différentes. $1^{0}$ Les denx moiliés s'acrolent par lemr bord externe sur la lignemédiane de manière à former une masse cellulaire axiale, se continuant latéralement endeur lames, l'une cetodermique, l'aut'e endodermique. $2^{\circ}$ Les deux couches pui contiement le bourrelet se fusionnent avant la réunion des deux moitiés du bourrelet sur la ligne médiane, pour constitucr après celte réunion une masse de cenlules indifférentes qui pourront alors se différencier en cellules ectodermiques, mésodermiques et endodermiques.

La première hypothèse. est inadmissible, cal les coupes pratiquées en avant de la vésicule de Kupffer montrent l'axe nerreux, la corde dorsale avec ses cellules caracléristiques, les lannes mésordermiques et l'endoderme nettement différenciés. La seconde ne ne parait pas plus acceptable que la prenière, car il faudrait alor's supposer que les cellules embryonnaires du bourrelet se différencient, au noment même de la lusion des bords du bourrelel, en éléments histogéniques aussi différents que ceux qui constituent l'axe nervenx ef la corde dorsale.

On peut encor admettre que les deux moiliés du bourrelet 110 forment qur les parties litérales de l'ambryon, l'axe nerveux et la corle dorsale s'aceroissant sur la lignne médiane par intussusception. En faremr de celte hypothèse on peut invoquer l'observation de liyder (156) relative au diveloppement de l'Elecate canadensis. Col antenr at vu sur des coufs dont le blastoderme était sur le point de sé lermer, les deux moitiés du bourrelet se continuer distinctement avec l'embryon conme les deux branches d'un Y renversé. Cies deux noiliés du bourrelet présentaient une segmentilion en somites jusqu'ì une certaine distance en arrière de l'embryon. Dins l’angle aigu formé par les deux branches de l’Y, il y arait un espace losangique, lormé de cellules reposint sur le vitellus et dans lequel liyder pense avoir vu la vésicule de kiuffer et un cordon axial qu’il croit être la corde dorsale. Liatenr américin troure que son observation confirme hien la théorie de His ef Rauber; il reeonnait cependant que la masse cellulitre entre les leus moitiés du blastoderme est un peu embarassante. Pour lui, la segnenta 
tion en somites des bords du blastoderme est une simple accélération ou précipitation dans le mode habituel du développement. Il pense que le fait observé chez l'Elecate est une sérieuse objection à la théorie de Ballour qui fait accroitre l'embryon à partir du bord de blastoderme, sans l'adjonction d'éléments provenant du bourrelet. Cependant Ryder ne repousse pas entièrement cette théorie et il tend à la concilier arec celle de la concrescence; il est probable, dit-il, que l'embryon s'accroit à la fois par intussusception d'arrière et en avant à partir du bord du blastoderme et par coalescence des deux moitiés de ce dernier, non sur la ligne médiane, mais par une fusion graduelle comme se ferme le blastopore sur le vitellus.

La formation de la partie postérieure de l'embryon par l'adjonction du bourrelet blastodermique est indiscutable. Tous les auteurs ont constaté qu'au moment de la fermeture du blastoderme, le hourrelet constitue en arrière du bourgeon caudal un petit anneau plus ou moins elliptique, dont le grand axe est dirigé suivant l'axe de l'embryon. Les bords de cet anneau se rapprochent de la ligne médiane en même temps que le grand diamètre diminue; ils se fusionnent et forment une masse cellulaire qui se soude intimenent au bourgeon caudal, de manière à ne laire qu'un tout homogène. Il est très facile de suivre cette fusion sur des coupes longitudinales ou transversales de la fin du stade $\mathrm{H}$, telles que celles que nous avons déjà décrites (Planches, fig. 97 et 98).

Je rapellerai que lorque les deux moitiés du bourrelet blastodermique se réunissent sur la ligne médiane, au dessous de la tente formée par la membrane enveloppante, les deux masses ectodermiques se soudent pour constituer une masse unique de cellules indifférentes qui émet de chaque côté une lame endodermique primaire s'étendant à une certaine distance au dessous de l'ectoderme. On peut suivre pas à pas, sur des coupes, Ia lusion des deux bords du bourrelet. Si une lusion semblable avait lieu, dans l'hypothèse de la concrescence, en avant de la vésicule de Kupffer, on devrait retrouver dans cette région la même disposition des cellules et des feuillets qu'on trouve là où se fait réellement la fusion. Nous avons déjà dit que cette disposition n'existait pas en avant de la vésicule de Kupffer, il faut done admettre que seule l'extrémité postérieure de l'em- 
bryonse forme par coalescence des bords du bourrelet, en arrière du bourgeon candal, au niveau du trou vitellin.

La théoric de His étant contraire aux faits d'observation, les lypothèses de Kupfler et d'OAillacher restent seules en présence. Toutes deux sont compatibles avec le résultat de mes recherches sur le mode daccroissement de l'embryon.

Il est difficile, sinon impossible, d'établir le véritable mode d'extension du blastoderme sur le vitellus d'après les observations faites sur les cufs des Salmonides ou tout autre œuf de Poisson de forme sphérique. Mais Miecz. von Kowalewski (103) me paraît avoir heureusement tourné la difficulté en prenant comme objet d'étude les øufs de forme ellipsoïdale du Carassius auratus et d'une espèce pélagique, indéterminée, provenant de Trieste. Sur ces cufs, dans lesquels les extrémités des axes inégaux de l'ellipsoïde servent de points de repère, von Kowalewski a constaté que, pendant la segmentation jusqu'au moment de la différenciation des feuillets embryonnaires, le blastoderme s'accroit également par toute sa périphérie, suivant le schéma de Kupffer; mais que, lorsque les feuillets sont constitués, l'extrémité eaudale de l'embryon reste fixée sur un point du vitellus, et que, par conséquent, à partir de ce moment l'extension du blastoderme se fait d'après le schéma d'OEllacher. Il me parait très probable qu'il en est de mème pour les cufs des autres Poissons et en partieulier des Salmonides.

\section{XII. - Conshembations gévérales.}

La comparaison des premiers stades de l'évolution des Poissons osseux avec ceux des autres Vertébrés, et surtout des autres Poissons, a déjà été faite par plusieurs auteurs; je rappellerai ici brièrement les faits connus et je n'insisterai que sur les points encore en discussion.

L'œuf des Téléostéens est un œuf méroblastique comme celui des autres Poissons; la segmentation y est donc partielle et n'intéresse que le germe. La formation des cellules aux dépens du parablaste montre que la substance plastique n'est pas nettement séparée du vitellus nutritif, comme on le pensait autrefois. Les recherches de Balfour (8) pour les Pla- 
giostomes, do Sirrasin 164) jour los lieptiles, et fle Waldeyer (193 pour les Oiseanx, nut prouvé yn’il en est do même pour les eul's méroblistiques des autres Vertébrés. et que des cellules, qui viennent s'ajouler aux sphères de segmentation du germe, prennent nilissmee dans le, vitellus au pourtour et andessous du gelme. Dans les auls ì segmentation inégale des Amphibiens. Jes Gimö̈des et des Gyelostontes, lit substance plastique est plus abondante of plus intimement mélangéc ì la substance vitelline, "l boutr la masse des grosses spheres de segmentation doit dure assimilér à la couche parablastique des aufs méroblastiques. Dans cos auf.s, cette masse de cellules parablastiques jouc un rôle important pour la formalion de l'mubryon. puisqu'elle constilne me partie de l'endoderme; chez les Sauropisides et les Pligiostomes, la couche parablastique perd déji de son importance et ne prend quine laible part it la formation de l'embryon: chez les Téléostéens, le parabliste est cneore moins développé el n'entre pas dans la constilution de l'embryon, à part les quelques cellules quil fournit au germe segmenté pendant un temps plus ou moins long. suivant les espèces. On pent done le considérer comme une sorte dorgane atavique représentant la masse dos grosses sphères de srgmentalion des cuufs des Cyclostomes et des Ganoïdes (1).

Les deux premiers stades de la segmentation noffrent rien de particuliop clez les Poissons osseux, mais présentent de l’intérèt par suite de lat relation que certains auteurs ont cru trouver entre lis direction du purmier sillon de segmentalion el la direction ultéricure do l'emhryon. Suirant Rauber (141142), chez la Grenomille. le premier sillon do segmentation divise l'ouf en deux moities dont l'une correspond à la future région céphalique de l'embryon, l'autre à la future région caudale: le premier sillon de segmentation sorait fone, d'après lui, perjendiculaire ì l'axe de l'embryon. Pllüger (126), Ronx (151, bis), ont une manière de roir loul à lail différente; pour eux, le premier sillon est dirigé suivant l'axe longitudinal de l'embryon.

(1) Iu point de vue de la segmentation, l’ouf du Lépinlostée, dont le développement a ćlé suivi d'une manière incomplète par Balfour et Parker (10), est celui qui se rapproche le plus de celui des Téléostẻens. Chez le Lépidostée, la masse ritelline ne subri qu une framentation imparfaite. 
Agassiz et Whitman (1) Mhnelful quw, ehez le Ctenolabrus, l. premier sillon est antéru-pustérieur; an rontraire, Fusapi (52) a vil que, cluez le Cristiceps argentulus, le premier sillon est dirigé snivant lr pelit diametre de l'ollipse du germe; cet anteur se range à l'opinion de limubre pour la Grenouille, el pense que le premier sillon est lansrepsal par rapport à l'mobryon. de nai pu faire ancune observalion de ee genre sur les wufs que jai ens a ma disposition; les wufs des Silmonides sont trop volumineux pour pouroir elpe ulservés par transparence ot se développent trop lentenent pour quil soit possible de suive un germe, depuis le début de la segmentation jusquà l'apparition de l'embryon. Le's wufs do l'Epinoche, quoique transpareuts, présentent aussi l'inconvenient d’évoluer lentement. En présence des résultats contritdictoires Rauber et de Fusari, d'une part, de Pllïger, Roux, Agassiz et Whitman, d'aulre part, on roit qu'il ost impossible actuellement d'établir une relation entre la direction du premier sillon de segmentation et eelle de l'ase de l'embryon. Je ne crois pas, du reste, que la solution de celte question présente une importance capitale, parce qu'on ne peut en tirer une conclusion générale.

Dans ses belles recherehes de tératogéniè oxpérimentale sur les Ascidies, Chabry (32) a ćtabli sûrement que le premier flan de segmentation est le plan méridien de la larve; en détruisant l'un des deux premiers blastomères, il a toujours ohtenu des demi-individus. L'observation de Clıabry vient done ì l'appui de celles de l'flüger of Rous, d'Agassiz ot Whitman: mais, d'un autre coté. Ilallez (64) a vu que, chez l'Asearide lombricö̈de, le premier sillon sépare. le futur ectoderme du futur endoderme, at que le second sillon divise l'ectoderme en une partie céplaalique etiune partie caudale. On sait anssi que, chez beaucoup d'autres Invertébrés, le premier plan de segurntation divise l'œuf en dem moitiés inégales, dont l'une est l’origine de l'endoderme, l'autre l'origine le l'ectoderme. Enfin, je rappellerai que, suivant C.-K. Hoffmann (89), le premier plan de segmentation, chez certains Poissons, sépare le gerne du parablaste. On voit done que, en adnettant comme démontrée, ce qui me paraît prématuré. l'existrnce, pour unr nême espèce̊ animale, d’une relation constante entre le premier plan de 
segmentation et l'axe de l'embryon, on n'en peut tirer aucune loi générale.

La direction de l'axe embryounaire ì la surface du blastoderme ehez les Salmonides ne commence à devenir visible que lorsque le germe segmenté présente à sil périphérie une partie plus épaisse. Sur des æufs de Carassius auratus, von howalewski (103) a pu reconnaitre la portion épaissie déjà an stade IV et an stade VIII. Il a reconnu que la segmentation marchait plus vite à la partie postérieure de l'embryon qu’ì la partie antérieure, et qu'on pouvait ainsi déterminer de bonne heure l’axe du futur embryon.

Le stade VIII, chez les Téléostéens, par la disposition particulière des sillons, a été jusqu’à présent regardé comme spécial ì ces animaux. Une observition intéressante de Ryder (160) a montré cependant que cette manière de voir était trop evelusive. L’auteur américain a constaté, en effet, sur l'œuf de la Raia erinacea l'existence d'un stade VIII, identique à celui les Téléostéens; les sillons de troisième ordre étaient parallèles au premicr sillon et perpendiculaires au second. Du reste, les variations que jai notées dans les premiers stades de la segmentation du germe de la Truite prouvent quil ne faut pas attacher une trop grande importance à la disposition des premicrs sillons.

Il n'existe pas, chez les Salınonides, decavité de segmentation comparable ì celle de l'Amphioxus, des Cyclostones, des Ganoïdes et des Amplibiens. Chez les Plagiostones, il apparait tardivement unc cavité dans l'épaisseur dufeuillet profond; cette cavité ne tarde pas à se transformer en cavité sous-germinale ou germinative. L'œuf des Sauropsides neprésente pas de cavité de segmentation bien nette. M. Duval (44) a décrit chez les Oiseaux une fente horizontale linéaire, au dessous de l'eetoderme, s'cffacant presque aussitòt après son apparition, et qüil considére comme une cavité de segmentation. La cavité germinative est, au contraire, bien développée comme clız les Poissons osseux.

Certains embryologistes, guidés pardes idées théoriques, ont distingué avec soin la cavité de segmentation de la cavité germinative. Cette distinction me paraît un peu subtile. Les deux espèces de cavité se forment, en effet, par un processus identi- 
que; l'une et l'autre sont produites par une accumulation de liquide dans un espace compris entre les sphères de segmentation et résultant de la disposition que prennent ces sphères.

La seule différence qui existe entre la cavité de segmentation et la cavité germinative, e'est que la première apparait de très bonne heure, dès les stades IV et VIII, tandis que la seconde ne se forme que plus tard, vers la fin de la segmentation. Lit cavité de segmentation est située entre les cellules ectoilermiques et les cellules endoderniques, la cavité germinative entre la face profonde du germe et le parablaste; celui-ci représentant la masse endodermique, il en résulte que les deur cavités occupent dans l'œuf une position identique.

La formation des feuillets embryonnaires amx dépens du germe segmenté, telle que je la comprends, cliez les Téléostéens, peut-clle être comparée à celle de ces mêmes feuillets, chez les autres Vertébrés? Yalgré les travaux nombreus et considérables publiés depuis quelques années sur l'embryogénie des Vertébrés, l'état de nos connaissances sur l'origine des teuillets rst encore trop peu avancé pour qu'une senmblable étude puisse être entreprise avec fruit. Les mêmes divergences d'opinion que jai signalées, relatives à lat formation des feuillets cliez les Poissons osseux, se retrouvent pour les autres classes de Vertébrés; elles ont été résumées récemment par Assaky (2) dans un travail spécial. Aussi me bornerai-je ici à examiner si le résultat de mes recherches sur les Salmonides permet de rapprocher le développement de ces animaux de celui des autres poissons,

A la fin de la segnentation. le germe des Téléostéens se présente sous la forme d'une calotte, dont les bords epaissis reposent sur le vitellus, ou plutôt sur le parablaste, et séparéc de ce parablaste, sur le reste de son étendue, par la cavitégerminative. Il est comparable, sous cette forme, à celui des Plagiostomes, lorsqur la cavité de segmentation s'est transformée en cavité germinative. Il correspond au toit de lí cavité de de segmentation des Ganö̈des, des Cyelostomes, formé par les petites sphères de segmentation; le parablaste, ainsi que jel’ai dit plus hat, est J'homologue des grandes sphères de segmentation. Le gerne des Téléostéens représente aussi la blastula 
delimphioxus, qui serait barsenent ouverte a sa partic indérieure, dans la future région endodermique, et dont lourerture cmbrasserait une partic du globe ritellin.

Lorsque se produit il la périphérie du germe la réflesion de l'eetoderme qui donne naissance à l'endoderme primaire, le blastodisque des Poiscons osseux est encore comparable à celni des autres Poissons, mais nous rogons apparaitre des differences assez notables dans le mode de formation de l'endoderme. Chez les Plagiostomes, le blastoderme présente en un point de si périphérie. comme chez les Téléostéens, un épaississement à l'endroit où apparaitra l'enbryon. Ciest le rebord terminal de l'embryon de Balfour (8). Le rebord forme parr l'ectoderme réfléchi et par les eellules de segmentation indifférentes est soulevé au dessus du vitellus, il en résulte qüil existe une cavité entre la face inférienre du rebord et le parablaste sous-jacent. Ce rebord, suivant lil généralité des embryogénistes, représente la portion dorsale de la lèrre du blistopore de J'Amphioxus, des Cyclostomes el des Ganoürles: Ja cavité est lintestin primordial (protogaster de Hiteliel) (Lrdarm, des auteurs allenands mésentéron de Palfour').

Chez les Téléostéens la partie embryomnaire du bourelet blastodemicque, homologue du rebord terminal de l'rubrron des Pagiostomes, ne se soulère pas au dessus du vitellus, et reste appliquéc sur lo parablaste; il n’existe done pas ehez ces animanx de carité de l’intestin primordial. J’ai démontré, en effet, l'erreur de Cunningham (34) qui a roulu roir dans la résicule de liupfer l'homologne de la cavilé comprise culpe le rebord terminal et le parablaste chez les Plagiostomes. La gastrula des Poissons osseux est, comme l'a bien vu llarcliel, une véritable discogastrula (qui, par son mode de formation el par sa constilution, se rapproche beaueomp plus de la gastrula type de l'Amphioxus que celle des autres Poissons.

Si lon suppose, en effet, la blastula de l'Amphiores onverte

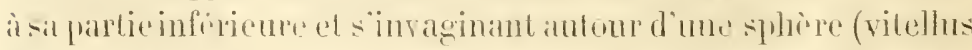
et parablaste) on anra me image exacte de la gastrula des Téléostérns a a mesure que la blastulas sétond à la surfice de la sphère. lạ partic invaginéc de lectoderme desecend ì la surlace de celle-ci mais les hords libres des deux replis dinvagination ne peurent se rejoindre au lond de la gastrula, par suite de la jrésence de 
la spltère vitelline et de l’atcéoissement rapido de l'ectoderne au póle supérieur de l'coul'. Lintestin primordial, le protogáasler, est rempli par liı masse vilelliue, et la bonche de la gastrula (anus de Rusconi) est lo blastopore vitellin, qui se ferme en arrière de l'embryon, au stade $\mathrm{H}$, chez les Salmonides.

Si la gastrula des Poissons osseux est voisine de celle de l'Amplioxus, la formation ultérieure de l'embryon, anx dépens de la gastrula, s'éloigne plus de eclle du type de l'Amphioxus que la formation de l'embryon des aul'rs P'oissons. Chez ceux-ci le protogaster prend part directement ì la formation de l'intestin définitif; elıez les Téléostéens, au contraire, la cavilé digestive est une formation secondaire et résulte d'une invagination de la cavité virtuelle de la gastrula. L'embryon de l'. mprioxus, des Plagiostomes, des Cyclostomes et des Ganö̈des, de même que cełui des Amphibiens, se développe sur le bord de la houche de la gastrula el la gouttière médullaire communique avec le protogaster par la lève dorsale du blastopore. Cependant, chez les Plagiostomes, Balfour (8) a montré que l'embryon cesse bientot d'atro en rapport avec le blastojore, par suite de la fermeture partiello de ce dernier, en atrrière de l'embryon, avant que le blastoderme ait recouvert lis totalité du vitellus. On sait que Balfour a assimilé lat bandelettr. résultant du rapprochement des lèves du hlastopore en arrière de l’embryon, à la ligne primitive des Vertébrés supérienrs, at que eette manière de voir a éte adoptée par la plupart des embryogénistes. Chez les Plagiostomes, lit ligne prinitive se forme donc après l'embryon, tandis que clez les Amuiotes clle précede lapparition de l'embryon.

liembryon des Téléostéens est en rapport, comme celui des autres P'oissons avee le hourrelet blastodemique, e'est-it-dire arec la lèvre du blastopore. Mais le sillon médullaire superfieiel, lomologue de la gouttière médullaire des autres Vertébrés, n’arrive jambis, ainsi que nous l'arons vu, jusqualu blastopore; il en est toujours séjaré par le bourgeon caudal. Celui-ci ocenpe done, par rapport à l'embryon, la même situation que la bandelette postembryonnaire des Plagiostomes, at que la ligne primitive des Amniotes. Le bourgeon caulal appitrait de très bonne hrure, dès de slade 1b, pent-itre mène plus tôt, en tout cas avant liapparition du sillon médullaire. 
D’un autre côté, jai montré que la structure du bourgeon caudal était identique à celle de la tête de la ligne primitive des Vertébrés supéricurs. Toutes ces raisons mont conduit ì admettre, dès 188\%, (75) que la formation de l'embryon des Téléostéens était précédée de celle d'une ligne primitive très réduite, représentée par le bourgeon caudal. Depuis lors, toutes les observations que jai faites sont venues corroborer cette opinion. Parmi ces observations, j'en citerai une qui me parait avoir une valenr particulière.

Sur un blastoderme du stade A, détaché du vitellus et exíminé par transparence dans le baume dn Canada, j’ai vu, sur le bord externe de la partie la plus épaissie du bourrelet blastodermique, c'est-ì-dire au point où apparait le bourgeon caudal. une petite encoche à peine marquée. Les cellules marginales du bourrelet convergeaient vers le sommet de l'encoche, et. leur disposition indiquail qu'il existait en cet endroil une petite invagination de la levre du blastopore vers le centre du blastoderme. de n`ai pu malheureusement répéter cette observation, parce qu'il est très difficile de séparer le blastoderme dı vitellus en lui conservant l'intégrité de ses contours. Je ne puis done dire si l'existence de l'encoche est normale an stade A. Si la présence de cette inragination était constante, clle indiquerait qu ïl se produit de très bomne heure, à la partie postérieure du futur embryon, un phénomène semblable à cehii qui sobserve au niveau de la bandelette postembryonnaire des Plagiostomes, ì savoir la fermeture d'une petite portion du blastopore. Quant ì l'épaississement du bourrelet blastodermique constituant le bourgeon caudal, il résulte évirlemment d'une prolifération active des cellules embryonnaires au niveau de l'invagination.

L'applarition de l'embryon des Téléostéens est donc précédlée, comme celle des Amniotes, de la formation d'une ligne primilive; tandis que chez ees derniers, la ligne primitive résulte de Ja fermeture conplète du blastopore; chez les Téléostéens, clle n'est constituéc que par une sorte de repli à peine marqué, des hords du blastopore; le blastopore vitellin se confond avec la bouche de la gastrula, et se ferme exactement en arrière de la ligne primitive (bourgron caudal) comme chez les Plagiostones. Chez les Amniotes, au contraire, le blastopore ritellin 
on ombilical, est distinet du blastopore vrai, ou bouche de la gastrula. Enfin, chez l'Amphioxus, les Gyclostomes et les Amphibiens, il ne se forme pas de ligne primitive proprement dite, puisque la bouche de gastrula persiste jusqu'ì la fermeture du canal neural, et que colui-ci communique avec la cavité de la gastrula far le canal neurentérique. On ne saurait, en effet, considérer comme une ligne primitive ce que miss Johnson (92) décrit comme représentant cet organe chez le Triton; la fusion des feuillets blastodermiques, en avant de la lèvre antérieure du blastopore, résulte de la constitution même de cette lèrre produite par la réflexion de l'ectoderme : ce qui représente chez les Amphibiens et les Cyclostomes la ligne primitive c'est, ainsi que l'a dit M. Duval (42), en 1880, le blastopore tout entier; mais ce blastopore ne se ferme que tardivement, lorsque l'embryon est déjà bien développé, et si, au moment de sa fermeture, il se présentesous la forme dune petite fente pouvant être alors assimilée à ume ligne primitive, celle-ci n'a qu'une existence très courte, et en tout cas est postembryonnaire comme celle des Plagiostomes.

La conception de la ligne prinitive des Poissons osseux, celle que je viens de formuler, est absolument différente de celle de Kupffer (106) que j'ai exposée précédemment. Cet auteur considère, en effet, comme ligne primitive le sillon médullaire primaire qui serail, d’après lui, une invagination cctodermique. J'ai réfuté sa manière de voir, je n'y reviendrai pas ici. Kollmann (100) admet aussi l'existence d'une ligne primitive chez les Téléostéens. Suivant lui, cette ligne primitive serait représentée par une petite dépression linéaire qui existerait entrè le sillon médullaire et le bourgeon catudal : celui-ci ferait également partie de la ligne primitive. Son opinion se rapproche done de celle de Kupffer, mais suivant lui la ligne primitive serait moins étendue. Kollmann a observé cette dépression sur des embryons de Sammon; je n'ai jamais rien ru de semblable chez la Truite. L'existence de la dépression serait-elle démontréc chez le Sammon, de même que chez le Brochet, d'après Kuptfer, el chez d'autres Poissons, il ne s'en suirrait pas qu'elle puisse être assimilée à la ligne primitive; elle indiquerait seulement que, chez certains Poissons, il se produit une invagination secondaire de l'ectoderme, analogue à celle qui donne 
naissance chez les Tertébrés supérienrs an canal neurentérique (1).

L'évolution et la signification du camal neurentérique des Vertébrés est encore très obscure. Chez l'Amphioxus, les Cyelostomes, les Ganoïdes, les Plagiostomes et les Amphibiens, ce canal est une formation promaire; il résulte du développement du sillon médullaire en rapport direct avec le blastopore. Mais chez les Amniotes, il parait être une formation secondaire. Si, en effet, le canal neurentérique peut être considéré théoriquement comme la partie antérieure de la ligne primitive, c'est-idire la partie de la bouche de la gastruliı en rapport avec la gouttière médullaire, on sait cependant par les recherches de Gasser et de Braun chez les Oiseaux de Balfour (9) et de Strahl (181-184) chez les Reptiles, que le canal neurentérique n'apparaìt généralement chez ces animaux qu’à une période avancée du développement et qüil peut y avoir formation successive de plusieurs canaux neurentériques. On est done en droit de se demander, si dans ce cas, le canal neurentérique ne proviendrait pas d'une invagination tardive de l'ectoderme mettant en communication le système nerveux avee la cavité digestive. Un phénomène semblable pourrait se produire chez les Poissons osseux en avant de la ligne primitive (bourgeon caudal), au niveau de la vésicule de Kupffer, comme paraissent le démontrer les observations de Kupffer.

Le canal neurentérique résulterait alors de la formation d'une gastrula sccondaire, absolument distincte de la gostrula primitive ou discogastrula. Suivant eette manière de voir, la discogastruli devrait être considérée comme une forme larvaire analogue ì la larve des Echinodermes, par exemple, sur laquelle se développe ensuite l'embryon proprement dit.

Je n’insisterai pas davantage sur ces considérations théoriques, qui, je le répète, étant donné l'état actuel de nos connaissances sur les premiers phénomènes du développement des Vertrébrés, ne peuvent conduire à aucune généralisation sé-

(1) Dans une note publice en 1880 (69) je considérais cette invagination secondaire comme l'homologue de l'intestin primitif des Cyclostomes et des Amphibiens, et son orifice exterieur, que javais constate chez la perche, comme représentant l'anus de Rusconi. On voit, d'aprés ce qui précède, que j’ai modifie ma manière de voir. 


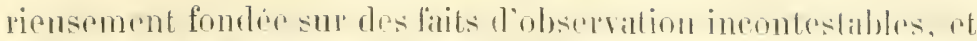
jo reviens a la bormation des fenillets embryomates chez les Poissons.

Lorsque l'ectoderme s'est réflechi ponr formar l'endoderme primaire et qu'apparait le premier molinent ('mbryonuaire, celui-ci a la même conslitulion chez les Poissons osseux que chez les Plagiostomes, les Cyclostomes et les Ganoïdes, mais tandis que, chez ces derniers, l'endoderme continue ì se développer dans sa portion ventrale de la cavité digestive, alix dépons des cellules parablastiques, ou des grandes celiules de segmentation, chez les Téléostéens le feuillet interne primaire est définitivement constitué et ne reçoit plus de cellules parablastiques. Chez tous les Poissons, l'mdoderme primaire se dédouble ensuite en mésoderme ut en corte dorsale. La différenciation du feuillot moyen et de la corde dorsale se fait simultanément d'arrière en avant, à partir de la lèvre du blastopore, chez les Plagiostomes, les Cyclostomes et les Ganoüdes à partir du bourgeon caudal chezles Télénstéens. Les deux lames mésodermiques apparaissent isolément de chaque côté de la corte ilorsale.

Chez l'tmphiores, on sait, depuis les belles recherehes de howalevsky (101) et de Hatsehek 65), que les masses mésodermiques at la corde dorsale premnent naissance sous forme de trois diverticulmms creux de l'endodermo. Les frères Hertwig (80) ont voulu retrouver un mode de développement semblable chez les autres Vertibrés. La théorie du coolone, résuméc par 0. Hertwig dans son Lehrbuch der Entwicklungsyeschichte, 1886, rifficile à adnettre pour les Plagiostomes, ne me parait pas pouvoir être appliquée aux Téléostéens. Chez ces animans, les lames mésodermiques résultent d'une délamination de l'endoderme prinaire, el en aucun point de l'enbryon on ne roit se produire de direrticules ereux, ni de plis d'invagination de l'endoderme. La cavité générale du corps, le colome, comme celle de la carité digestive, rst une formation secondare : clle se produit dims l'éparisseme de chaque lanne mésodepnique, par simplo éeartement des deux comeles eellulaires. Liexistence d’un mésenclyme, cost-ì-elipe d’un lissu formé de reflutes atmiboüles dre provenaneres diverses, est, am

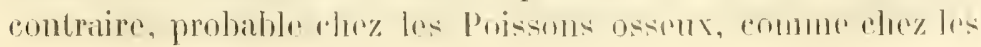


autres Vertébrés. J'ai indiqué, en effet, la présence de cellules migratrices dans diverses régions, entre autres dans la tête, où le tissu conjonctif et ses dérivés semblent provenir de cellules détachées du mésoderme et de l'endoderme primaire. En tout cas, je ne saurais accepter l'origine parablastique des cellules du mésenchyme, origine soutenue par Waldeyer(193), Rückert (150-151) et Hertwig (79-80). Les cellules du mésenchyme des Poissons osseux ont pour origine des feuillets, provenant des cellules de segmentation.

Je ne reviendrai pas ici sur le développement des différents organes. Nous avons vu que chez les Poissons osseux, clıacun des trois feuillets donne naissance allx mêmes organes que chez les autres Poissons et les autres Vertébrés. Mais le processus de formation d'un certain nombre de ces organes est différent. Ce qui caractérise le développement de l'embryon des Téléostéens, e'est ce que j'appellerai le développement massif, par opposition au développement par invagination, qui s'observe chez presque tous les autres Vertébrés. Le développenent massif, qui consiste dans la formation des organes par un épaississement local ou par l'apparition d'un bourgeon plein de l'un des feuillets du blastoderme, est surtout remarquable pour le système nerveux. La formation de l'axe nerveux des Poissons osseux est précédée, comme chez les autres Vertébrés, de l'apparition d'un sillon, d'une dépression, à la surface de l'ectoderme. Nais le processus d’invagination est tout à fait transitoire et est bientòt remplacé par un épaississement du feuillet externe. Le développement massif du système nerveux a pour conséquence la formation secondaire de la cavité de l'axe nerveux; il en est de même pour la vésicule optique, la vésicule auditive, la partie moyenne de l'intestin, les protovertèbres, la tige sous-notochordale, etc.

Le développement massif du système nerveux se retrouve chez les Cyclostomes et le Lépidostée; il y est cependant moins marqué que chez les Téléostéons. Calberla (31) et Scott (172) ont montré, en effet, que dans l'embryon de la Lamproie, l'ectoderme se divise en deux couches, dont l'externe s'invagine dans le cordon neural et qu'une lente médiane au centre de l'involution représente la gouttière médullaire des autres Vertébrés. Chez le Lépidostée, la cavité du système nerveux 
apparait plus tôt que chez les Téléostéens; mais, à part quelques légères différences, de peu d'importance, le développenent du Lépidostée est celui qui ressemble le plus à celui des Poissons osseux; malheureusement la formation des feuillets b]astodermiques est encore inconnue chez cet intéressant Ganoïde, qui, tant au point de vue anatomique qu'au point de vue embryogénique, constitue évidemment une lorme de passage des Ganoïdes aux Téléostéens.

Dans une note relative à l'origine de l'amnios, Ryder (159) fait jouer, un rôle important à la pression exercée par l'enveloppe de l'œuf pour empêcher le développenent de l'amnios chez les Vertébrés inl'érieurs (1).

Je ne discuterai pas cette théorie qui peut, en effet, otre acceptée provisoirement pour éclairer l'histoire encore obseure de l'origine de l'amnios; mais je ferai remarquer qu'on peut également invoquer cette pression exercée par la capsule de l'œuf pour expliquer le développrement massif des Téléostéens. La capsule de l'œuf est inextensible; j’ai indiqué à plusieurs reprises les altérations qui se produisent dans la forme du germe ou de l'embryon, lorsque ceux-ci se trouvent comprimés entre le vitellus et la capsule, sous l'influence des agents fixateurs. Cette pression interne, exagérée dans ce cas, existe cependant normalement; c'est elle qui s'oppose à la production des invaginations qui ont lieu dans les oul's des autres Vertébrés, chez lesquels la membrane vitelline manque ou est très mince et extensible, et chez lesquels l'embryon est séparé de la coque rigide de l'œuf par une couche plus ou moins abondante d'albumine. Il est à noter, en effet, que chez le Lépidostée, l'enveloppe de l'œuf est également épaisse et résistante; celle de l'œuf de Lumproie parait, il est vrai, assez mince et rappelle celle des Batraciens anoures; mais, de mème que chez les Téléostéens, l'œuf n'augntente pas de volume et l'embryon est obligé de s'enrouler dans l'intérieur de l'œuf. Malgré sá

(1) His (84) a décrit de chaque côté du corps des jeunes emloryons de Plagiostomes un repli à peine marqué qu'il considère comme un repli amniotique rudimenlaire. Rien de semblable ne s'observe clıez les Téléostéens; la loordure embryonnaire des premiers stades indique simplement la région didermique ou tridermique du blastoderme, et par conséquent, la limite externe de la bordure est la limite même de l'embryon. 
minceur, l'onvoloppe de l'wenl des Cyclostonues serait plus résistante que celle des Anphihiens el sopposerit au déreloppement, it lit formation du systeme nerreus par inragination crense de leetuderme.

Qne l'luppothese que je viens de formuler soit fondéc on non, que la pression exercéc par la coque de l'ouf sur l'entbryon soit la ciuse du déreloppenent massif ou que celui-ci dépende d'une antre canse eneore inconnue, je ne range entièrement is l'opinion de Balfour (9), (p. 28:3), qui considère la formation du système nerveus central aux dépens d’un épaississement en carène de l'ectoderme comme un mode dérivé et secondaire, et le reploiement de la plaque médullaire en un canal, comme le proeessms printitil. Levistenee du sillon superficiel chez les Téléostéens, précédant l’épaississement ectodemique, pronve que linvagination est le type primitif de développenent du système norveux. On peut en dire autant des antres formations massives des Téléostéens.

En résuné, on voit que l'mubrygénie des Poissons assemx offre un grand intérèt, parce qu'elle nous fait connaitre un processus particulier de développement qui sépare nettement ces animaux des autres Poissons. Si les trails généramx de ce développenent sont communs ì toutes les sons-clisses des Poissons, la constitution de l'ceuf, la formation de la gastrula, la présence diune ligne primitive rudimentaire, la constitution primordiale du système nerveux et de quelques antres organes indiquent que le groupe des Poissons ossenx représente une branche divergente du phylum des Poissons. Les données mmbryogéniques corroborent colles que nous fournit l'anatomie comprare: elles montrent que, si sous certains rapports, les Téléostéens constituent un type dégradé des Poissons, on trouve cependant chez cux les premiers indices des caractères distinctils des Vertébrés supérieurs.

de ne remx pats insister davantage sur les conelusions plytogéniques quon peut tirer de l’étude du développement des Poissons osseux. Je me suis attaché dans ce travail it exposer ansifi fidelement que possible les résultals que m’itdounés une observation attentive of somvent répetée des promieres phases dre l'mobryogénie de la Truite. Jai rherché avant tout ì etablir

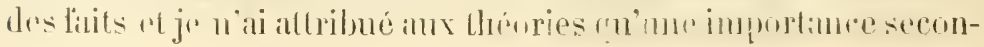


ditire, me rappelant les sages paroles de l'un de nos plus illustres embryogénistes, du furofrssum licelliker : « Lembryologic n’a rien de miens ì laire qu à suive provisolrement sil propre route et, laissant de còlé les hypolhèses plyylogénétiques, à šefforeer de décourrir les lois de la formation des organes, des appareils et des organismes entiers. Cette tàche accomplie, la comparaison du développement des individus et des diverses espèces permettra de sélever à la connaissance des lois générales du développenunt des organismes el, peu ì peu aussi, par le progrès de l'examen rationnel et pliilosophlique de cette embryologie comparée, une science de la descendance, saine et prudente, verra se poser sa base et se continuer son údifice. Trop de préeipitation et de témérité à se jeter dans cette direction rencuntre de tous côtés l'éeueil et peut créer du danger par les tentatives de diverses sortes qu'elles font naître. Cortainement la science a plus d’intérèt à laisser d'abord les faits se constituer : si, dans le domaine de l'embryologie, ils sont le prix d'efforts plus pénibles, les fruits n'en sont aussi que plus doux à cueillir. " (97) (p. 40 et 411$)$.

I'u et approuvé,

Paris, le 12 novemlre 1888 .

Le Doyen de la Faculté des seienees,

E. IIÉBERT.

Tu et permis dimprimer,

Le Vice-Recteur de l'Académic de Paris.

Le 12 Novembre 1888.

GREARI). 


\section{INDEX BIBLIOGRAPHIQUE}

1. Agassiz, A. and Whitmax, C.0. - On the development of some pelagic Fischeggs. Preliminary notice. Proceed. of the American Acad. of Arts and Sciences XX, 1884.

2. Assaki, G. - Origine des feuillets blastodermiques chez les Vertébrés. Paris, 1886.

3. Aubert. - Beiträge sur Entwickelungsgeschichte der Fische. Zeitschrift für wissensehaftliche Zoologie, VII, 1850.

4. BaER, C. Erxst vox. - Entwickelungsgeschichte der Fische. Leipzig, 1838.

5. - Entwicklungsgeschichte der Thiere. Th. I. Künigsberg, 1828.

6. Balbiav, G. - Sur la constitution du germe dans loeuf animal avant la fécondation, Comparaison de ce dernier avec l'ovule végetal. Comptes rendus de l'Acad. des seiences, mars et avril, 1864.

7. - Leçons sur la genération des Vertébrés. Paris, 1879.

8. Balfour, F. 11. - A monograph on the development of Elasmobranch Fishes. London, 1878.

9. - Traité d'embryologie et d'organogénie comparée. Paris, 1883-85.

10. Balfour, F. M., and PARKER. W. N. - On the development of Lepidosteus. Philosoph. Trans. Roy. Soc., London, CLXXIll, 1882.

11. Banbeke C. Vay. - Recherches sur l'embryologie des Poissons osseux. Mém. cour. et mém. étrangers de l'Acad. des śc. de Belgique, XL, 1876.

12. - Recherches sur l'embryologie des Batraciens. Bull. de l'Acad. roy. de lielgique, LXI.

13. Baungertver, M. - Beobachtungen iiber die Nerven und das Blul. Freilourg, 1830.

14. BeARD, Johs. - On the segmental sense organs of the lateral line; and on the morphology of the Vertebrate auditory organ. Zool. Anzeiger, VII, 1884́.

15. - The origin of the segmental duct in Elasmobranchs. Anatomischer Anzeiger, II, $n^{\circ} 21,1887$.

16. Ведот. - Recherches sur le developpement des nerfs spinaux chez les Tritons. Recueil zoologique suisse, 1884.

17. Belloxci, Jos. - La caryocinèse dans la segmentation de l'œuf de l'Axolotl. Archives italiennes de Biologie, VI, 1884.

18. Bexedex, Eb. Vax. - La maturation de l'œuf, la fécondation et les premières phases du développement embryonuaire des Mammifères. Bull. de l'Acad. de lielgique, 2 e serie, $\mathrm{XV}, 1875$.

19. - Contribution à lhistoire du développement embryonnaire des Teléosteens. liull. de l'Acad roy. des Sciences, des lettres et des Beaux-Arts de lielgique, 2* série, ẊLIV, 1877.

20. - Recherches sur la maturation de l'auf et la fécondation (.Iscaris megalocephala). Archives de Biologie, IV, 1883.

21. liéRaxEck, E. - Recherches sur le developpement des nerfs crinienschez les Léaurds. Recueil zool. suisse, I, 185'. 
22. - Étude-sur les replis médullaires dı Poulet. Recueil zool. suisse, IV, $188 \%$.

23. Biscuof F, L. W. - Entwicklungsgeschichte des Hunde-Eies. Braunschweig, $18 \mathbf{4 5}$.

24. Borretsky, N. - Studien über die embryonale Entwicklung der Gasteropoden. Arch. f. mikr. Anatomie, XIII, 1876.

25. Brook, G. - Preliminary account of the development of the Lesser weever fish (Trachinus vipera). Limnean Soc. Journal; Zoology XVIII, 188́.

27. - On some points of the development of Motella mustela. Linnean Soc. Journal: Zoology, XVIII, 1885.

26. - On the origin of the Iypoblast in Pelagic Teleostean Ora. Quart. Journal of microscop. Science; new Series, XCVII, 1885.

28. Brtcn. - Ueber die Befruchtung des Thiereies. Mainz, 1855.

29. Bütsculı, 0. - Studien viber die ersten Entwicklungsvorgänge der Eizelle, die Zclltheilung u. Conjugation der Infusorien. Abandl. d. Senkenbeig. Naturf. Gesellsch., X, Frankfurt, 1876.

30. Calberla, E. - Befruchlungsvorgang beim Ei von Petromyzon Planeri. Zeitschrift. f. wiss. Zool. XXX, $187 \%$.

31. - Zur Entwickelung des Medullarrohres und der Chorda dorsalis der Teleostier und der Petromyzonten. Morphol. Jahrbuch. III, 1877.

32. Chanry, L. - Embryologie normale et tératologique des Ascidies. Journal d'Anat. et de Physiol., 1887.

33. Coste. - Origine de la cicatricule ou du germe chez les Poissons osseux. Comptes rendus de l'Acad. des Sciences, XXX, 1850.

33 bis. - Histoire générale et particulière du développement des corps organises. Paris, 1847-59.

34. Cuxwıgнам, J. T. - The Significance of Kupffer's vesicle, with Remarks on other Questions of Veriebrate Morphology. Quari. Journ. of mic. Science; new Series XCVII, 1885.

35. - On the Relations of the Yolk to the Gastrula in Teleosteans and in other Tertebrate Types. Quart. Journ. of. mic. Science: new Series CI, 1885.

36. Dorryxiv, P. V. - Ueber die erste Anlage der Allantois. Sitzungsber. d. k. Akad. Wiell, LXIV, 1871.

37. Dohr., A. - Der Ursprang der Wirbelthiere und das Princip des Functionsuechsels. Leipsig, 1875 .

38. Dorène. - Tote sur l'œuf du Loligo media et sur celui du Syngnathe. Journal de l'Institut, 1850, et Socièté philomathique, 1849.

39. Drrsy. E. - Der Primitivstreif des Hühnchens, Lahr, 1867.

40. - Entwicklungsgeschichte des Kopfes. Tübingen, 1869.

41. Duval, Matitus. - Étude sur l'origine de l'allantoïde chez le Poulet. Revue des Sc. naturelles, VI, 1877.

42. - Études sur la ligne primitive de lembryon du Poulet. Ann. des Sc. naturelles, $6^{\circ}$ série, VII, 1880.

43. - Segmentation, globules polaires. Comptes rendus de la Société de Biologie, $7^{\mathrm{e}}$ série, IV, 1883.

44. - De la formation du blastoderme dans l'auf d'Oiseau. Ann. des Sc. naturelles, 1884́.

45. Etsig, H. - Die Segmentalorgane der Capitelliden. Mittheil. a. d. zool. Station zu Neapel, I, 1879.

46. FilıPPI, DE. - Memoria sullo sviluppo del Ghiozzo d'aqua dolce (Gobius fluviatilis). Annali univ. di Medic. compilati dal dott. Umodei, 184 l.

47. Fuemwg, W. - Zellsubstanz, Kern-und Zelltheilung. Leipzig, 1882.

48. - Ueber die Bildung von Richtungsfiguren in Sïngethiereiern beim Untergang Graaf'scher Follikel. Arch. f. Anat. und Entw. 1885.

49. - Die ectoblastiche Anlage der Urogenilalsystems beim Kaninchen. Arch. f. Anat. u. Entw., 1886. 
50. Fot. H. - Recherches sur la ficondation et le commencement de l'hinogenie. Mèm. de la Sac. de phys. et dhist. nat. de Geneve. 1879.

51. - Recherches sur le développement des protovertèbres chez l'embryon de Poulel. - Archives des Scjences phys. et nat. XI. Genève, 1884.

52. Fussri, R. - La segmentazione nelle uova dei Teleostei. Bolletino del XII. Congresso medico. Pavia. 1887.

52 bis). (iasser. - Veber Entwickelung der Allanlois, der Mibller" schen Gïnge und des Afters, lrancfort, 1874.

53. Gegenbarr. C. - Ueber den Bau und die Entue. der Wirbellhiereier mit Dotter. Müller's Archiv. 1861.

5ú. Gexscn. Ilego. - Das selundiure Entoderm und die Blutbildung beim lii der Knochenfische. Inaugural-Dissertation. Königsberg, 188².

55. Genne, Z. - Du lieu ou se forme la cicalricule chez les Poissons osseux. Joumal de l'inat. et de la l'lrysiol., XI. 185̃.

56. Gerlacu. L. - Ueber die endodermale Entuickelung der Chorda dorsalis. biologisches Centralb/att. 1I, 1881.

57. Gorovowitscn, X. - Studien viber die Entwirlilung des Medullarstranges bei Knochenfischen nebst Beobachtungen über die erste Anlage der Kémblatter und die Chorda bei Salmonider. Jior'h. Jalnbuch, X, is85.

5S. Gнтte, G. - Beitrige zur Entuichlungsgeschichte der Wirbelthiere. Der Keim des Forellen-Eies. Arch f. milir. Anat. IX, 1873.

59. - Entwicklungsgeschrchte der Lnke. Leipzig. 187'́

60. - Ueber die Entwicking des Central-Nervensystems der Teleostier. Archiv. f. mikr. Anat. XV, 1878.

61. Grosnex, C. - Die Entuiclielungsgeschichte der Moina rectirostris. Zngleich ein Beilrag zur Kennlniss der Inatomie der Phyllopoden. Arbeiten aus d. zool. Institut d. Univ. Wien. Il, 1879.

62. Grigrinn, L. - Recherches anatomirues at physiologirnes sur l'embryon des Légumineuses. Ann. des Sc. Nat. Bot. $6^{\circ}$ serie, Xll, 1S8:.

63. IIECKEL, E. - Die Gastrula und die Eifurchung der Thiere. Jenaische Zeitschrift. 1X, 1875.

6í. Mallez. - Recherches sur l'embryologie des Nimatodes. Paris, 1885.

65. Hatscheк, B. - Studien über Entwicklung des Amphioxus. Arbeiten aus d. Zool. Institut der Univ. Wien. IV, 1881.

66. Ilfaps, W. - The development of the Hole (Talpa Europea). The formation of the germinal Layers, and early development of medullary (ironve and Votocorde. Quari. Journ. of mic. Science; new Sèries XCI. 1883.

67. Hexinerity, L.-F. - Becherches sur la vitulite des spermalozö̈des de la Truite. Comptes rendus de l'lead. des Sc. 187.

68. - Procéde technique pour l'étude des embryons de Poissons. Bull. de la Soc. philomathique, 1878.

69. - Note sur quelques faits relalifs an $x$ premiers phinomenes du développement des Poissons osseux. Bull. de la soc. philomathique, 1880.

70. - Formation du germe dans l'ouf des Poissons osseux. Bull. de la Soc. de liiologie, 188u.

71. - Division des cellules embryonnaires chez les Jertètrés. Comptes rendus de l'Acad. des sc., 188:.

72. - Formation des cellules embryonnaires dans le parablaste des poissons osseux. Bull. de la Soc. de Biologie. 1S5:.

73. - Sur la dirision celluluire ou eylodierèse. Assoc. fr. pour lavancenent des :c. Congres de La Rochelle, 188*.

74. - Sur la formation des fenillets embryonnaires cher la Truite. Comptes rendus de l.lcad. des Sc., 188?.

75. - Sur la ligne primitive des Poissons osseur. Zoologischer Inzeigrer, 185.5.

76. - Sur le mode d'accroissement de l'mbryon des Poissons osseux. Comples rendus de l'deademie des Sc., $185 \%$. 
7. Hexsex. - Beoluthungen ïber die Befruchung u. Entwirking des Veerschueinchens u. Kuninchens. Zeitsch. I. Inat. u. Entw, 15is.

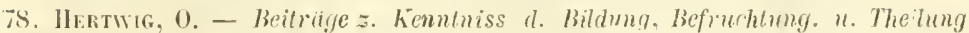
d. Thier-Eies. Holphologisches darhluuch, 1, 11, 111. 1875-76-77.

7!). - Die Entuick?ung des milleren Keimblalles der ITirbelthiere. Jonaische Zeitschrif: f. Naturw. XV et XVI, 1881-8*.

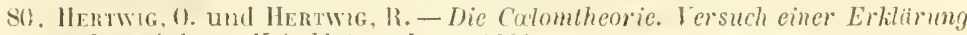
des milleren Keimblattes. Jena, 18s1.

81. HIs, W. - Untersueliungen über die erste Anlage der Wirbelthierleibs. Leipzigi, 1868.

82. - Untersuchungen ïher das Ei und die Entwicliehng bei knochenfischen. Leipzig, 1873.

83. - Lntersuchngen über die Entwicklung von Knochenfischen. Zcilscit. f. Anat. und Entwicklung. 1, 1876.

8\{. - Ueber die Bildung von Ilaifischembryonen. Zeitschr. f. Anat. u. Entw., 187.

85. - Intersuchungen üher die Bildung des Knochenfischembryo. Arch. f. Anat. und Entwick., 1878.

86. - Veher die Anfinge des peripherischen Nervensystems. Arch. 1. Anat. u. Entwick. 1879.

87. Horfusx, C. K. - Zur Onlogenie der Knochenfische. Zool. Anzeigrer, $187 \mathrm{~S}$.

85. - Leter die Entuichelungsgeschichte der Chorda dorsalis. IIenle's Festgahe. Bonn, 188.2.

89. - Zur Ontogenie der Knochenfisehe. Verhandelingen d. K. Alad. der Wetenschappen. Amsterdam, 1881-1883.

y0. - Zur Ontogenie der Knochenfische. Arch. f. mikrosk. Anat. XXII, 1883.

91. Javosik, J. - Partielle Furchung bei den knochenfischen. Arch. I. mikr. Anat. XXIV, 1834 .

92. Jonssox, ALAeE. - On the fate of the blastopore and the presence of a primilif streak in the Newt. Quarterly Journ. of micr. Science, 188 i.

93. lingstey and Conx. - Some Observations on the Embryologie of the Tcleosts. Mem. lioston society, III, 1883.

94. KuEs, E. - Obsernations on the Earty Development of the common Trout (Salmo fario). Qualt. Journal ol microscop. Scrence; new Series XVI, 1876.

9.. Kolessinkow. - Ueber die Entwickelnng bei Batrachiern und Knochenfischen. Areh. 1. mik. Inat. Xiv, 1878.

96. KuELLLER. A. - Untersuchungen zur vergleich. Gewebelehre angestell zu vizza in llerbst 18,36. Verhandi. d. phys. med. Gesellschaft zu Würzburg. 18.,8.

97. - Embryologie ou traité complet du developpement de l'Homme et des animanx superien's. Trad. par A. Schneider. Paris, 188:.

95. Kollusx, J. - Der Randwh'st und der Ursprung der Stützsubstanz. Areh. f. Anat. u. Entw. 1884.

99. - Gemeinsame Entwickelungsbahne der II irbelhiere. Arch. I. Anat. u. Entw. 188.5.

100. - Die Geschichte des Primitivstreifens bei den Meroblastiern. Verhandl. d. Naturf. Gesellsch, in Basel, 1886.

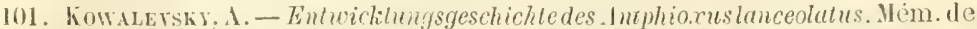
l'Aearl. $r$ des Sciences de Saint-Petersbourg. 7 serie. XI. lsci.

102. Kowalewsil, Mecz vos. - Die Gastrulation und die sogen. Allantois bei den Teleostiern. Silzungsber. d. physili-ned. Societät zu EPlangen. 1886 .

103. - Uber die crsten Enlwicklungsprocesse der Knochenfische. Zeilsch. f wiss. Zoologic XLIII, 1856.

104. Kuprer, C. - Beobuchiungen ïher die Enluisklung der Knochenfische. Arehir, 1. mikrosk. Analonic 11. 1868. 
104 (bis). - Die Entstehung der Allantois und der Gastrula der Wirbelthiere. Zoologischer Anzeiger II. 1879.

105. - Das Fi con Arvicola arvalis. Sitzungsber. d. bayr. Ahad. d. Wissenschaft, 188:.

106. - Die Gastrulation an den meroblustischen Eiem der Wirbelthiere und die Bedeutung des Primitivstieifs. Arch. f. Anat. u. Entwicklungsgeschichte, 1882-1884.

107. - Primäre Metanerie des Neuralrohrs der Vertebraten. Sitzungsb. d. k. b. Akad. d. Wiss. zu München, 1885.

108. - Die Befruchtung des Forelleneies. Bayerische Fischerei-Zeitung, 1886.

109. KupfFer und Bexecke. - Der Vorgang der Befruchtung am Fi der Neunaugen. Königsberg, 1878.

110. Laxg, E. - Kernfurchungen. Virchow's Archiv. LIV, 1871.

111. Lereborleet, M. - Recherches sur le développement du Brochet, de la Perche et de l'Écrevisse. Annales des Sciences nat. $4^{\mathrm{c}}$ séric I, $185 \mathbf{4}$.

112. - Recherches d'embryologie comparée sur le déreloppement de la Truite, du Lézard et du Limnée. Annales des Sciences nat. $4^{\circ}$ serie XVI. 1861.

t13. - Recherches sur les monstruosités du Brochet. Ann. des Sc. nat. XX. 1863.

114. Levckst. - Article Zeugung in Wagner's Handwörterbuch der Physiologie. 1V, 18.53.

115. Lieberktenx. N. - Ueber die Keimblïter der Säugethiere. Zur 50jährigen Doctor-Jubelfeier des Ilerm llermann Nasse. Marburg, 1879.

116. List, Jos. H. - Zur Heikunfi des Periblastes bei Knochenfischen Labriden). Biolog. Centralblatt, VIl, n. 9, 1857.

117. - Zur Entwicklungsgeschichte der Knochenfische (Labriden. Zeitseh. f. wiss. Zoologie. XLY, 1887.

118. Miescier. - Cité dans le mémoire de llis, 83 ).

119. Munes Marsinal. - On the early stages of development of the nerves in Birds. Journ. of Anat. a. P'hysiol., XI, 1877.

120. Muellen, J. - Ueber zahlreiche Porencanile in der Eikapsel der Fische. Mï̈ller's Archiv., 1854.

121. Nuel, P. J. - Recherches sur le developpement du Petromyzon Planeri. Archives de Biologie. II, 1881.

122. Gulacner, J.-Beiträge zur Geschichte des Keimbläschensim Tirbelthierei. Arch. f. mikr. Anat., V111, 1872.

123. - Beitriige zur Entwicklungsgeschichte der Knochenfische nach Beobachtungein ain Bachforellenei. Zeitsch. f. wiss. Zoolog., XXII, 1872, et XXili, 1873.

124. Owsunsikow, PH. - Deber die ersten Vorgänge der Entwickelung in den Eiern des Coregonus lavuretus. Bulletin de l'Acad. Imp. de Saintpétersbouı. Xix, 1874.

125. - Studien ïber das Ei hauptsächlich bei Knochenfischen. Mèm. de I'Icad. de Saint-Pétersbours. XXX111, 1885.

126. Pflleger. - Ueber den Einfluss der Schwerkraft auf die Theilung der Zellen. Arch. f. d. ges. Physiol. XXXI, 1883.

127. 1'Révost, J.-1., et Dexas, J.-13. - Développentent de l'œuf des Batraciens. Ann. des Sc. nat. 11, 1824 .

128. Prince, Enw.-E. - The significance of the Iolk in the Eggs of Osseons Fishes. Annals and Magazine of nat. llistory, XX, 1887.

129. Rsmu, C. - Ueber die Bilding les Herzens der Amphibien. Morpholog. Jahrbuch. XII, 1886.

130. - Cité par IIertwig dans Lehrbuch der Fntwicklungsgeschichte.

131. Rabl-liteckhard. - Zur Deutung und Entwicke?ung des Gehims der Knochenfisch. Archis f. Anat. und Entwick. 1882.

132. liadwaver, J. - Ueber die ersle Anlage der Chorda dorsalis, Sitzungsh. Wien. Akatl. LXXIII, 1876. 
133. Rafaele. - Uova e larve di Teleostei. Bolletino della Societả dei Naturalisti in Napoli. I, 1887.

134. Ranson, W. H. - Observations on the Ovum of Osseous Fishes. Philosophical Transactions. 1868.

135. Ratuke, H. - Bildungs-und Enwickelungsgeschichte des Blennius viviparus oder Schleimfisches. Abhandl. z. Entw. 11, 1833.

136. Rauber. - Die erste Entwickelung des Kaninchens. Sitzungsb. d. Naturf. Gesellschaft zu Leipzig. 1875.

137. - Primitivstreifen und Neurula der Wirbelthiere. Leipzig, 1877.

138. - Die Lage der Keimpforte. Zoologischer Anzeiger, 1879.

139. - Foimbildung und Formstorung in der Entwicklung von Wirbelthieren. Morphol. Jalmbuch. V, 1879.

140. - Noch ein Blastoporus. Zoologischer Angeizer, 1883.

141. - Furchung und Achsenbildung bei den Wirbelthieren. Zoologischer Anzeiger. 1883.

142. - Nene Grundlegungen aur Kenntniss der Zelle. Morplool. Jahrbuch. VIII, 1883.

214 bis. Reicnert. - Das Entwicklungsleben im Wirbelthierreiche. Berlin, 1840.

143. - Ueber die Müller-Wolff'schen Kürper bei Fischembryonen und über die sogenamiten Rotationen des Dolters im befinchteten Hechteie. Müller's Arehiv, 1856.

144. - Ueber die Mikropyle der Fischtier und ̈̈ber einen bisher unbekannten, eigenthimlichen Bau des Nahrungsdotters reifer und befruchteter Fischeier. Müller's trehiv. 1856.

145. - Der Nahrungsdotter des 'Hechteies: eine kontraktile Substañ. Müller's Arehiv. 185\%.

146. Remak. - Untersuchungen über die Entwicklung der Wirbelthiere. Berlin 1850-1855.

147. Rieneck. - Ueber die Schichtung des Forellenkeims. Areh. f. mikr. Anat. V. 1869 .

148. Romit, G. - Studi di embriogenia. I. Contribuzione allo sludio dei foglietli embrionali. Rivista elinica di Bologna. 1873.

149. Rosenberg. 1. - Untersuch. ̈̈. die Teleostier-Niere. Diss. Dorpat, 1867.

150. Rueckert, J. - Zur Keimblattbildung bei Selachiem. Münehen, 1885.

151. - Ueber die Anlage des mittleren Keimblattes and die ersle Blutbildung bei Torpedo. Anatom. Anzeiger, Il, num. 4 et $6,1887$.

151 . is. Rocx. W. - Ueber d. Zeit d. Bestimmung d. Hauptrichtungen d. Froschembiyo. Leipzig. 1883.

152. Rusconı, M. - Erwiderung auf einige kritische Bemerkungen des Herrn von Baer über Rusconi's Entwicklungsgeschichte des Froscheies. Müller's Archiv, 1836.

153. - Ueber die Metamorphosen des Eies der Fische voi der Bildung des Embryo. Müller's Archiv, 1836.

154. RyDer, Jonx-A. - Development of the Silver Gar (Belone longirostris) with observations on the genesis of the blood in embryo Fishes, and a comparison of Fish ova with those of other Vertebrate. Publ. of the UnitedStates Fish Commission, 1, 1881.

155. - On the position of the Yolk-blastopore as determinated by the Size of the vitellus. American Naturalist, 1885.

156. - On the formation of the embryonic axis of the Teleostean-embryo by the concrescence of the rim of the blastoderm. American Naturalist, 1885.

157. - On the availability of embryological characters in the classification of the Cordeta. American Naturalist, 1885.

158. - The A rchistome-theory. American Naturalist, 1885.

159. - The origin of the Amnion. American Naturalist, 1886.

160. - On the earlier stuges of cleavage of the blastodisk of Raia erinacea. Bull. of the United States Fish Commission, 1886. 
161. Sabatier, A. - Sur les cellules du follicule et les cellules granuleuses che: les Tunicier's. Recueil zoologique suisse, 1, 188'́

16:. - Contribution à l'étude des globules polaires et des eléments élininés de l'auf en général (Théorie de la sexualite). Montpellier, 1S84́.

163. Salexskr. - Recherches sur le développement du Sterlet (Accipenser ruthemus). Archives de biologie, Il, 1881.

16́. Sarusix. - Reifung und Furchung des Reptilieneies. Arbeiten aus d. zoot. zool. Inst. in Würzburg, Vl, 1883.

16.5. Scinprixger, 1. - Ueber die Bildung des Medullarrohres bei den Knochenfischer. Stizungsb. d. K. Aliad. d. Wissensch. in Wien. 1l. Ahth. 1871.

166. ScHExk, S. L. - Zur Entwickelungsgeschichte des Auges der Fische. Sitzungsb. d. k. Akad. d. Wissensch, in Wien. 1867.

167. - Die Eier von Raja quadirimaculata innerhall der Eileiter. Sitzungsber. d. Lais. Akad. d. Wissensch. in Wien. 1873.

168. Schultz, A. - Beitiag zui Entwickelungsgeschichte der Knnorpelfische. Arch. f. mik. Anatomie, XIII, $187 \%$.

169. Scintmze, Max. - Das System der Circulation. Stultgart, 1836.

170. - De ovorum ianamm segmentatione. Bonn., 1863.

171. Schlutze, 0. - Unter'suchungen äber die Reifung und Befruchtung des Aimphibieneies. Zeitschr. f. wiss. Zoologie, XLV, 1886.

172. Sсотт, W. B. - Beitrige zur Entwickelungsgeschichte der Petromyzonten. Morphol. Jahrbuch, YH, 185..

173. SEDGWiGk-Mixot. - Sketch of comparative Embryology. American Naturalist. 1830.

174. SeEssel. - Zur Entwicklungsgeschichte des Vorderdarms. Arch. f. Anat. u. Entwick. $187 \%$.

175. SElkxks, E. - Keimblätter und Pimitivorgane der Maus, Wiesbaden, 1883.

176. - Die Blätterumkehrung in Ei der Nagethiere, Wlesbaden, 1884.

17\%. Semper, C. - Das Urogenitalsystem der Selachier. Arbeiten aus d. zoot. zool. Institut Würzburg, 11, 1876.

178. SHruey, - On some points in the development of Petromyzon fluviatilis. Quart. Journ. of. mic. Science, 1887.

179. Solger, B. - Dottertropfen in der intracapsularen Flüssigkeit von Fischeiern. Arch. f. mikr. Allat. XXVI, 1885.

180. Stockus, R. - Die äussere Eitzapsel der Forelle. Mittheil. embryol. Institut. Wien. II, 1883.

181. Strailu, H. - Ueber die Eutwichelung des Canalis myeloentericus und der Allanto is der Eidechse. Arch. f. Anat. u. Physiol. Anat. Abth. 1881.

182. - Beitiäge zur Entwickelung ron Laceita agilis. Arch. f. Anat, u. Physiol. Anat. Abth. 1882.

183. - Beiträge zur Entwicklung der Reptilien. Arch. f. Anat. u. Physiol. Anat. Abtl. 1883.

184. - Ueber Canalis newrentericus u. Allantois bei Lacerta viridis. Arch. f. Anat. u. Phys. Anat. Abth. 1883.

185. Strasburger, E. - Zellbildung und Zellheilung, Iena, 1880.

186. Stricker, S. - Untersuchungen äber die Entwichlung der Bachforelle. silzungsb. der Wiener h. Akad. d. Wiss. LI, 1865.

187. Trixcuese. - I primi momenti dell' evoluzione nei Molluschi, Atti d. R: Acad. dei Lincei, 1875.

188. Thossos, Allex. - Article Ovwin, in Todt's Cyclopedia of Anat. and l'hysiol. V.

189. Ussow, M. - De la sínucture des lobes accessoires de la moelle épinière de quelques Poissons osseax. Archires de biologie, 11], 1852.

190. Valexciexies el Frixy. - Recherches su la composition des oufs dans la série des animaux. Comptes rendus de licad. des sciences, XXXVlll.

191. Vogr, C. - Embryologie des Salmones. Neufchâtel, 1812. 
192. Walderer. - Eiersíock und Eii. Leipzig, 1870.

193. - Archiblast und Purablast. Aich. f. mikrosk. Anatomis, 1893.

19'. WaLden, I. - Ueber dus Verhalten der Zellkerne in den Furchungshugeln am Ei der Wirbelthiere. Berichte des Naturwiss. med. Ver. in Innsbruck, XI, Jahrg, 1881.

195. WEIL, G. - Beiträge zur Kenntriss der Kinochenfische. Sitzungsh. d. Wiener k. Akad. der Wiss. LXVI, 1872.

196. Wevchebacil, K. F. - The development of the blood corpuscules in the embryo of Perca furiatilis. Journal of Anat. a. Physiol. XIX, 1885.

197. - Beiträge zur Entwickelungsgeschichte der Knochenfische. Arch. I. mik. Anat. XXVIII, 1886.

198. Whituas, C. 0. - A rare form of the Blastoderim of the Chick, and its Bearing on the Question of the Formation of Vertebiate Embryo. Quart. Journ. of mic. Science : new Sieries XCl, 1883.

199. Wishe, J. W. VAx, - Die Betheilung des Ektoderms an der Entwicklung des Vomierenganges. Zoologischer Anzeigrer. N. 236, 1886.

200. Ziegler, E. - Die embryonale Entwickelung fon Salno Salar. Diss. Freiburg. B., 188:.

201. - Die Entstehung des Blutes bei Knochenfischenbryonen. Arch. f. mik. Anat. XXX, 1887.

202. - Ueber die Gustrulation der Teleostier. TagebIatt der 60. Versammlung deutscher Naturforscher und Aerzte zu Wiesbaden, n 8.1887.

\section{EXPLICATION DES PLANCHES}

\section{Planghe I.}

Fıg. 1. - OEuf ovarien de Truite, quelque temps avant la déhiscence du follicule : $v g$, vésicule germinative; $m$, micropyle. L'œuf est vu par transparence après avoir été traité par l'acide acétique. G $\frac{8}{i}$ :

F1g. 2. - OEuf de Truite, pris dans la cavité abdominale ou immédiatement après la ponte : $g$, germe; $h$, globules huileux. $G \frac{8}{i}$.

Fig. 3 - Fragment d'une coupe d'un ouf ovarien de Truite, dans la région du germe : $c h$, chorion; $v g$, vésicule germinative; $a$, globules plastiques finement granuleux; $b$, globules vitellins renfermant $h$, des grouttelettes huileuses. G $\frac{50}{1}$.

Fig. 4. - Fragment d'une coupe d'un ceuf de Truite au moment de la ponte : $c h$, chorion; $c c$, couche corticale; $h$, globules huileux. G $\frac{50}{1}$.

Fło. 5. - Globules vitellins d'un œuf ovarien de Gymnote, à diflérents états de développement.

FiF. 6 et 6'. - Otuĺs d'Epinoche, une demi heure après la fécondation, montrant les déformations du vitellus produites par la contraction de la couche corticale pendant la concentration du germe.

Fig. 7 à 24. - Vues superficielles d'œufs de Truite depuis la fécondation jusqu'à la fermeture du blastoderme. Les neufs, durcis par l'acide chromique, ont été dépouillés du chorion et dessinés à la chambre claire. $g$, germe; $b$, blastoderme ; $e$, embryon; v', vitellus; $v$ ', blastopore vitellin. $G \frac{4}{1}$.

Fig. 7. - Au moment de la fécondation.

Fig. 8. - Quatre heures après la fécoudation.

Fig. 9. - Fin de la concentration du germe. 
Fí. 10. - Segmentation du germe en quatre.

Fig. 11. - Segmentation avancée.

FIG. 1‥ - Fin de la segmentation.

Fig. 13. - Stade A.

FIG. I't. - Stade B.

Fis. 10. - Stade C.

Fig. 16. - Stade D.

Fig. 17. - Stade D'.

Fig. 18. - Stade E.

Fic. 19. - Stade F.

FIG. 20. - Stade G.

Fig. 21. - Stade G'.

Fug. 2.2. - Stade II.

Fig. 23. - Stade II'.

FIG. 24. - Embryon, lorsque le vitellus est entièrement recouvert par le blastoderme.

Fig. 25 à 46. - Différents stades de la segmentation du germe de l'œuf de Truite. Toutes ces figures ont été dessinées à la chambre claire sur des cufs fixés par l'acide chromique additionné d'acide acétique. Le vitellus sous-jacent au germe n'a pas été représenté. $G \frac{20}{1}$.

Fig. 23, 26 et 27 . - Apparition du premier sillon de segmentation.

Fig. 28. - Stade II, irrégulier.

Fı. 29. - Stade II, régulier.

Fig. 30 et 31 . - Commencement du stade IV.

Fir. 32. Stade IV, irrégulier.

Fig. 33. Stade $\mathrm{N}$, régulier.

Fig. 34. Stade $1 \mathrm{~V}$, raniforme.

liı. 33 et 36. - Stades VIII, irréguliers.

Fig. 3i. - Stade V'III, régulier.

Firs. 38 et 39. - Stades VIll, tout-ì-fait irréguliers.

Fig. 40. - Segmentation irrégulière.

Fig. 4l. - Stade XVI, régulier.

Fig. 42 et 43 . - Stades XVI, irréguliers.

Fig. 4 et et 40 . - Stades avancés de la segmentation.

Fis. 46. - Fin de la segmentation.

Fıg. 47. - Stade $\Lambda$; $a$, épaississement du bourrelet blastodermique correspondant au futur embryon; $b b$, bourrelet blastodermique. $\mathrm{G} \frac{20}{1}$.

Fig. 48. - Stade B. Portion du blastoderme comprenant l'écusson embryonnaire. $b c$, bourgeon caudal; $b b$, bourrelet blastodermique. G $\frac{20}{1}$.

\section{Planche Il.}

\section{SIGNIFICATION DES LET'TRES.}

a. Vésieule auditive.

hb. Bourrelet blastodermique.

be. Bourgeon caudal.

lie. liordure embryonnaire.

c. Cerveau. ca. Cordon axial.

cd. Corde dorsale.

rg. Cavile germinative.

c. Ectoderme.

$c p$. Ectoderme primaire. 
es. Ectoderme secondaire.

$k$. Vésicule de kuplfer.

$m$. Sillon médullaire.

$m n$. Segments métamériques lu cerveau portéricur.

ms. Mésoderme.

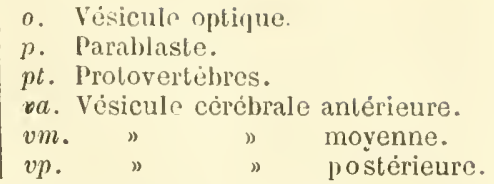

Fı. 49. - Stade C. Portion du blastoderme comprenant l'écusson embryonnaire. $\mathrm{G} \frac{20}{\mathrm{i}}$.

FIG. 50. - Stade C, dans lequel le sillon médullaire est largement ouvert en avant. $G \frac{20}{1}$.

Fig. 51. - Stade D. G $\frac{20}{1}$.

Fig. 52. - Stade D'. G $\frac{29}{1}$.

FiG. 53. - Stade E. G $\frac{20}{1}$.

FIg. 54. - Embryon du stade E, détachè du vitellus, coloré au carmin, monté dans le baume, et vu par transparence. $c$, carène nerveuse. G $\frac{20}{1}$.

Fic. 55. - Stade F. G $\frac{20}{1}$.

Fug. 36. - Embryon du stade F, détaché du vitellus, coloré au carmin, monté dans le baume, et vu par transparence. G $\frac{20}{1}$.

F16. 57. - Stade F'. G $\frac{20}{1}$.

Fug, 58. - Stade G. G $\frac{20}{1}$.

Fıg. 59. - Stade H. bv, hlastopore ou trou vitellin.

Dans les figures de 65 à $\$ 7$, les cellules n'ont pas été représentées sur les coupes. Le mésoderme a une teinte plus foncée que celle des deux autres feuillets. $\mathrm{G} \frac{25}{1}$.

Fıg. 63 à 74 . - Coupes transversales du stade C; les coupes sont indiquées dans l'ordre numérique d'arrière en avant.

Fig. 73. - Coupe longitudinale médiane du stade C.

FIG. 76 a 80 . - Coupes transversales du stade D; les coupes sont indiquées dans l'ordre numérique d'arrière en avant.

lig. 81. - Coupe longitudinale médiane du stade D.

Fig. 82. - Coupe longitudinale latérale du stade D.

Fig. 83. - Coupe longitudinale médiane du stade $\mathrm{F}$.

Fıg. 84. - Coupe longitudinale latérale du stade F.

Fıg. 85. - Coupe longitudinale du stade F, encore plus éloignée du plan médian.

1 IG. 86. - Coupe longitudinale médiane du stade $H$.

Fir. 87. - Coupe longitudinale latérale du stade H.

Fig. 88. - Coupe optique du bourrelet blastodermique d'un embryon đe Perche, observé à l'état vivant. $p$, parablaste.

Planche III.

SIGNIFICATION DES LETTRES pour les Fig. 60 à 64.

ce. Couche enveloppante.

$h$. Globules huileux.

nc. Noyaux des spheres de segmentation. $n p$. Noyaux du parablaste.

p. Parablaste.

$s$. Sillons de segmentation.

$v$. Vitellus, 
lis. 60. - Coupe transversale d'un germe de Truite au stade II de la segmentation. Acide osmique. $\mathrm{G} \frac{100}{1}$.

Fig. 61. - Coupe d'un germe au stade XVI. Acide osmique. G $\frac{100}{1}$.

Fig. 62. - Coupe d'un germe dont la segmentation est assez avancée. Liquide de Kleinenberg. G $\frac{100}{3}$.

Fig. 63. - Coupe d'un germe plus avancé. La couche enveloppante est déjà distincte à la surface du germe. $a$, cellule se détachant. du parablaste, Liquide de Kleinenberg. G $\frac{100}{1}$.

Fis. 64. - Fragment d'un germe détaché du vitellus avec le parablaste, et vu par transparence. Les cellnles du germe ont été en partie enlevées pour montrer le fond de la capsule parablastique. $\mathrm{G} \frac{100}{1}$.

\section{SIGNIFICATION DES LETTRES}

pour les figures 89 à 116 .

an. Axe nerveux.

bb. Bourrelet blastodermique.

bc. Bourgeon caudal.

c. Cerveau.

ca. Cordon axial.

$c d$. Corde dorsale.

ce. Couche enveloppante.

cl. Ccelome.

co. Cœur.

$\mathrm{cr}$. Cristallin.

e. Ectoderme.

$e^{\prime}$. Epiderme.

ep. Endoderme primaire.

es. Endoderme secondaire.

g. Globules sanguins.

gp. Globules parablastiques.

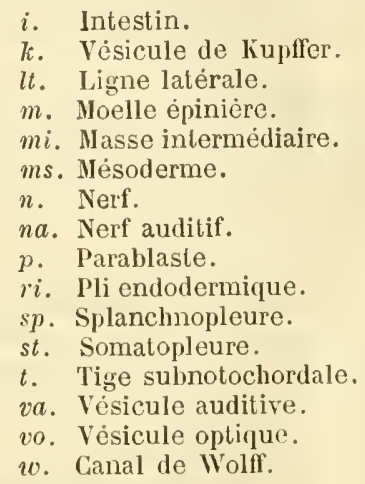

i. Intestin.

k. Vésicule de Kupffer

tt. Ligne latérale.

$m$. Moelle épinière.

$m i$. Masse intermédiaire.

na. Nerf auditif.

p. Parablaste.

ri. Pli endodermique.

$s p$. Splanchnopleure.

st. Somatopleure

$t$. Tige subnotochordale.

auditive.

w. Canal de Wolff.

Fig. 89. - Coupe longitudinale d'un germe de Truite au moment de la réflexion de l'ectoderme pour former l'endoderme primaire. (ca indique par erreur la couche enveloppante). G $\frac{100}{-1}$.

Fig. 90. - Coupe transversale du bourgeon caudal au stade C. G. $\frac{150}{1}$

Fig. 91. - Coupe transversale de la région moyenne d'un embryon au stade C. Cette figure représente la portion médiane de la fig. 69 plus grossie. $l$, lacunes dans l'endoderme primaire. $\mathrm{G} \frac{100}{1}$.

Fig. 92. - Partie médiane de la fig. 76 , montrant le cordon axial. Stade D. G $\frac{100}{1}$.

Fig. 93. - Partie médiane de la fig. 78, montrant la différenciation de la corde dorsale et des lames mésodermiques, aux dépens de l'endoderme primaire. Stade D. G $\frac{100}{1}$.

F1G. 94. - Portion médiane de la fig. S0, montrant l'extrémité antérieure du cordon axial. $\mathrm{G} \frac{100}{\mathrm{~T}}$.

Fig. 95. - Coupe transversale du stade $\mathbf{F}$, en arrière des vésicules auditives. $\mathrm{H} \frac{100}{1}$.

Fig. 96. - Coupe transversale du stade F, dans la partie postérieure de l'embryon. G $\frac{190}{1}$.

Fıg. 97. - Coupe transversale du trou vitellin, au stade H. G $\frac{100}{1}$. 
Fı. 98. - Coupe de la méme région, montrant la fusion des deux bourrelets blastodermiques. $\mathrm{G} \frac{100}{1}$.

\section{Plaxche IV.}

Fig. 99. - Coupe transversale du stade G, au niveau des vésicules optiques. G $\frac{100}{1}$.

Fig. 100. - Coupe transversale du stade $G$, au niveau des vésicules auditives. G $\frac{100}{1}$.

Fig. 101. - Coupe transversale du stade $G^{\prime}$, en arrière des vésicules auditives. $\mathrm{G} \frac{100}{1}$.

Fig. 102. - Coupe transversale du stade F', au niveau de la vésicule de Kupffer. G $\frac{100}{1}$.

Fis. 103. - Coupe transversale du stade $\mathrm{H}$, au niveau du pédoncule des vésicules optiques. $\mathrm{G} \frac{100}{1}$.

Fig. 104. - Coupe transversale du stade $\mathrm{H}$, au niveau des vésicules auditives. $\mathrm{G} \frac{\mathbf{1 0 0}}{1}$.

$F_{1 G}$. 105. - Coupe transversale du stade $H$, en arrière des vésicules auditives. $\mathrm{G} \frac{100^{\circ}}{1}$.

Fig. 106. - Coupe transversale du stade $\mathrm{H}$, dans la région moyenne de l'embryon. G $\frac{100}{1}$.

Fig. 107. - Coupe transversale du stade $H$, au niveau de la vésicule de Kupffer. $\mathrm{G} \frac{100}{1}$.

Fig. 108. - Coupe longitudinale de la partie postérieure d'un embryon du stade F. G $\frac{100}{1}$.

Fig. 109. - Coupe longitudinale de la partie postérieure d'un embryon à la fin du stade 11 , après la fermeture du blastederme. $b b$, bourrelet blastodermique soudé au bourgeon caudal. G $\frac{100}{1}$.

Fig. 110. - Portion médiane d'une coupe transversale du stade D, dans le milieu de l"embryon, montrant la disposition des cellules dans les différents feuillets. $\mathrm{G} \frac{150}{1}$.

Fig. 111. - Fragments d'une coupe sagittale du stade H, montrant les rapports du pharynx avec la vésicule auditive. $G \frac{100}{1}$.

Fig. 112. - Fragment d'une coupe transversale du stade G, dans la région moyenne de l'embryon montrant la différenciation de la lame mésodermique en protovertèbre et lame latérale. G $\frac{125}{1}$.

FiG. 113. - Coupe transversale dans la région du cerveau moyen, après la fermeture du blastoderme. $\mathrm{G} \frac{100}{1}$.

Fig. 114. - Fragment d'une coupe transversale du stade II, au niveau des vésicules auditives, montrant les deux rudiments cardiaques. $\mathrm{G} \frac{150}{1}$.

Fig. 115. - Fragment d'une coupe du même stade, montrant la formation d'une fente branchiale. $\mathrm{G} \frac{150}{1}$.

Fıı. 116. - Cavité du parablaste situé au-dessous de l'embryon et renfermant des globules parablastiques. $\mathrm{G} \frac{200}{1}$. 



\section{TABLE DES MATIERES}

InTRODUCtios. . . . . . . . . . . . . . I

PREMIERE PAR'TIE

I. - Constitution de l'œuf non fécondi...... . . . . 6

II. - Fécondation................ . 23

III. - Segmentation. . . . . . . . . . . . . 30

IV. - Formation des feuillets blastodermiques. . . . . . . 55

V. - Formes extérieures de l'embryon. . . . . . . . . 62

VI. - Etude des coupes des différents stades. . . . . . . 71

\section{DEUXIF́ME PARTIE}

VII. - L'ectoderme et ses dérivés. . . . . . . . . . . . . . . 91

VIII. - L'entoderme et ses dérivés. . . . . . . . . . . 113̈

IX. - Le mésoderme et ses dérivés. . . . . . . . . . . . 132

X. - Le parablaste et le sang. . . . . . . . . . . . 139

Xi. - Accroisssement de l'embryon.......... . 145

XII. - Considérations générales.. . . . . . . . . 157

IXdex bibLtograpHOUE. . . . . . . . . . . . . 172

Explichtox des planches. . . . . . . . . . 179

FIY DE L.I TABLE DES MATIERES. 


\section{ERRATA}

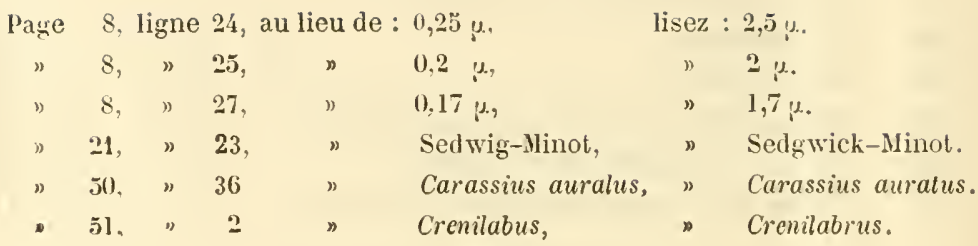




\section{DEUXIËUE TILSE}

PROPOSITIONS DONNÉES PAR LA FACULTÉ

ZooLogie. - Le type Insecte.

Botanique. - 10 Développement de l'embryon chez les Gymnospermes.

Grílogie. - Le terrain eocène. - Caractères de ses principales subdivisions.

Vu et appronvé,

Paris, le 12 novembre 1888.

Lo Doyen de la Faculté des seiences,

E. HÉBERT.

$\forall u$ et permis d'imprimer,

L.e Vice-Recteur de l'Académie de Paris.

Le 12 Novembre 1888.

GRÉARD. 




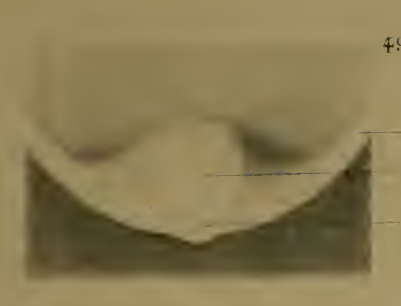

4!1
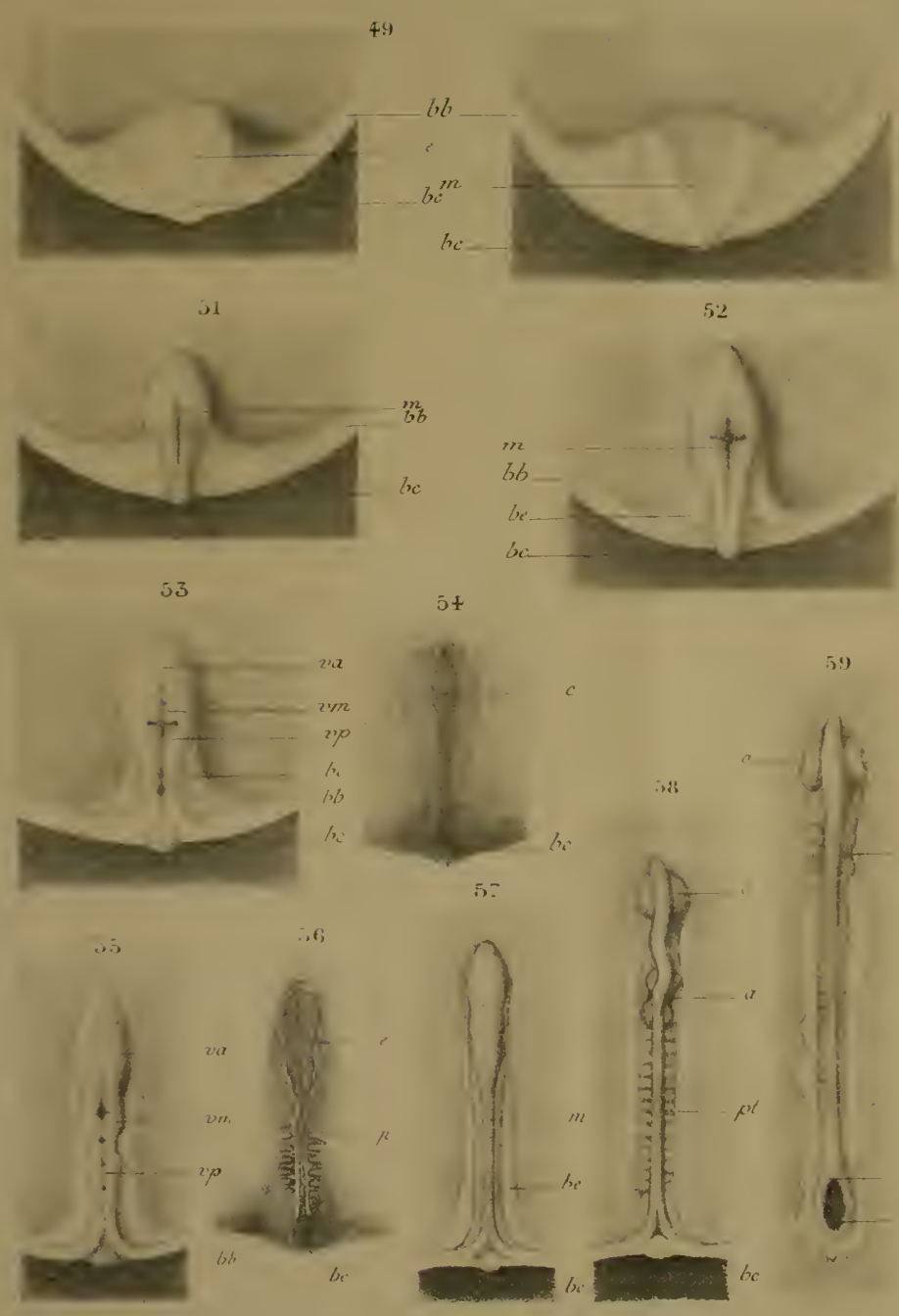

Ilenvosy is mui Le.

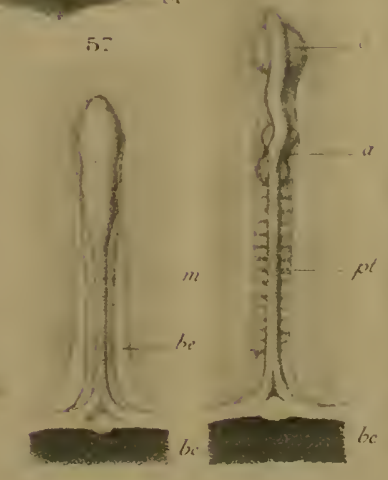

1
50
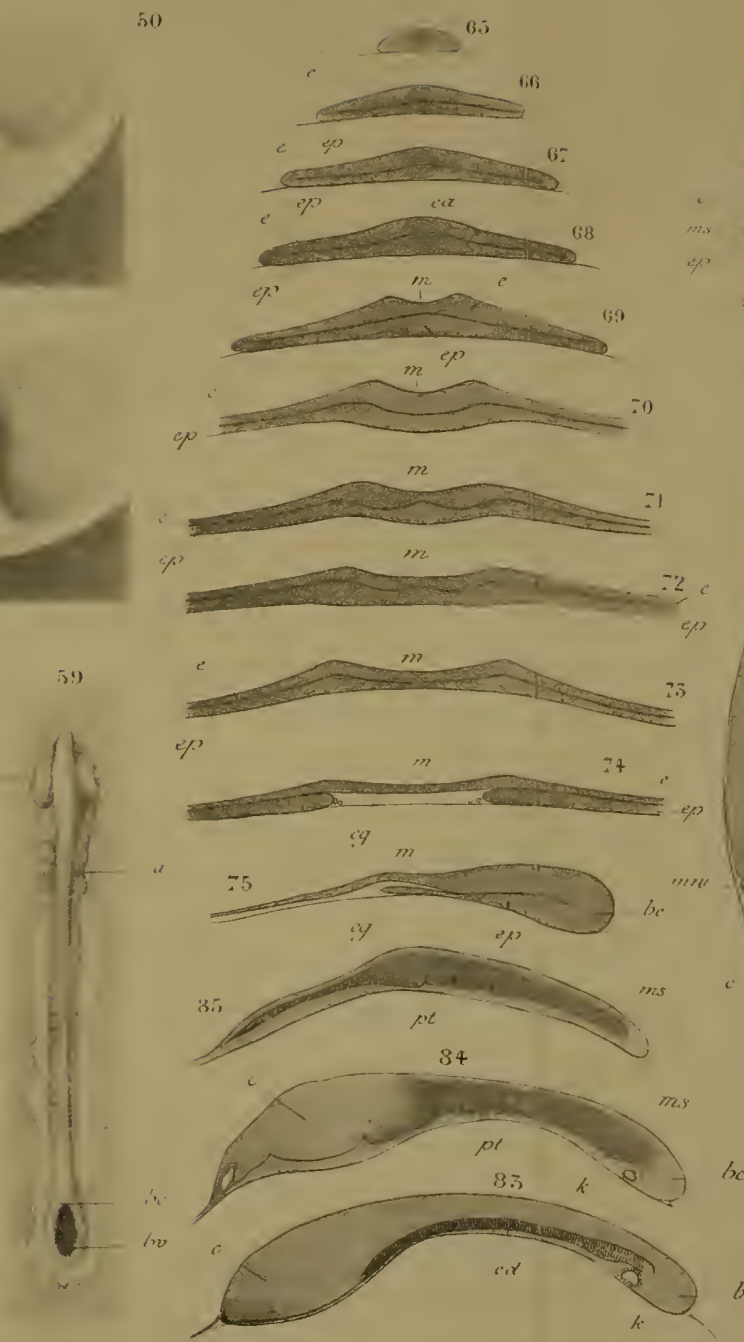

Impbecoud triarst

Wiai is is
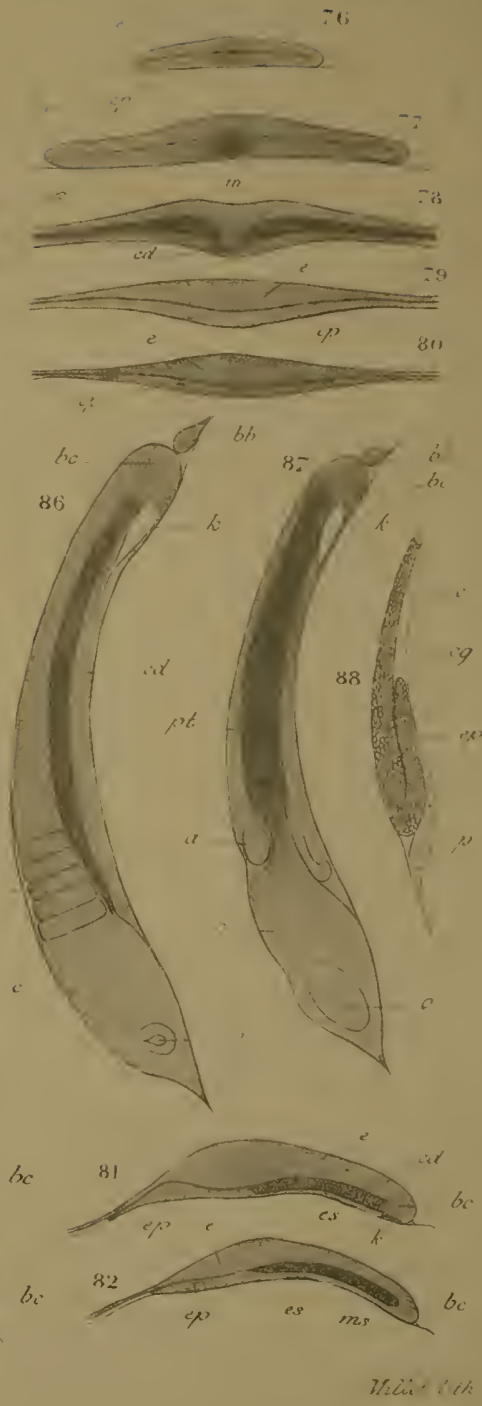

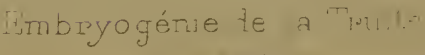





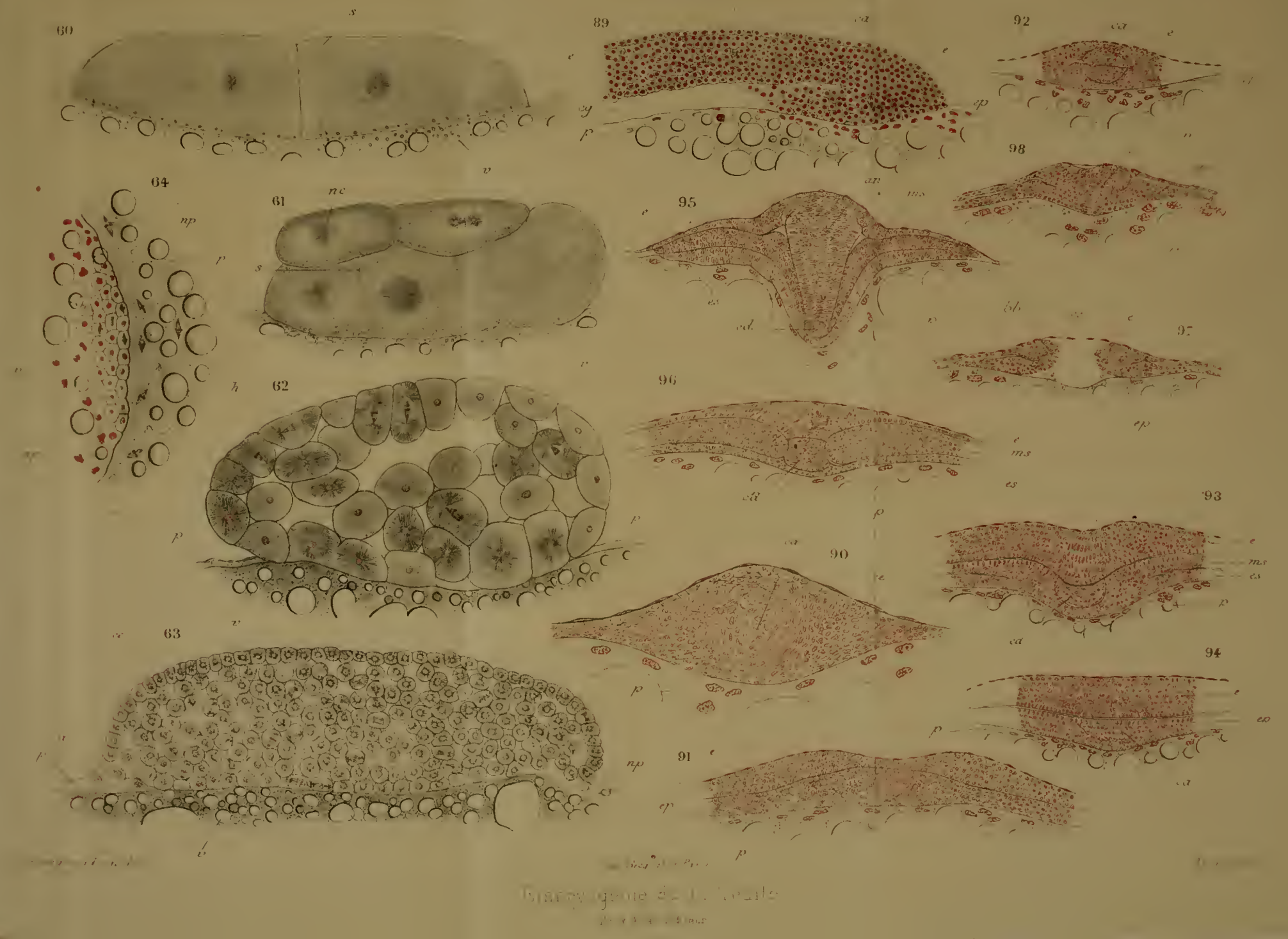




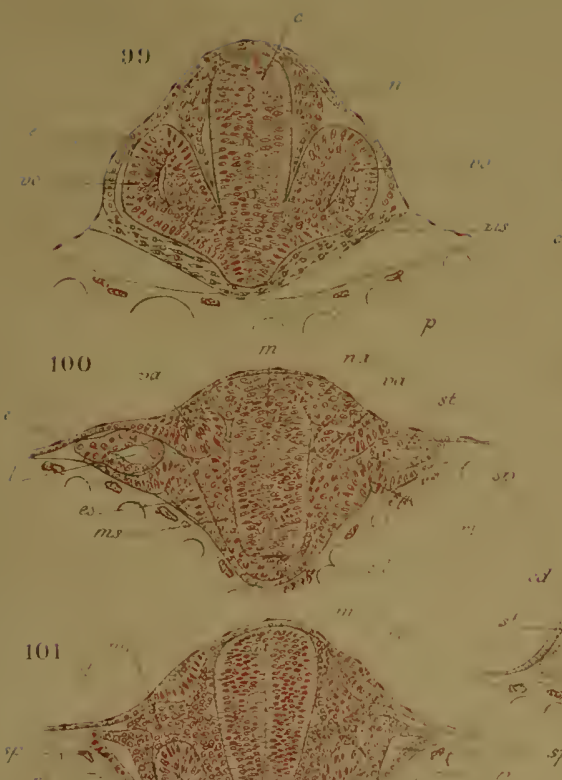

'"',

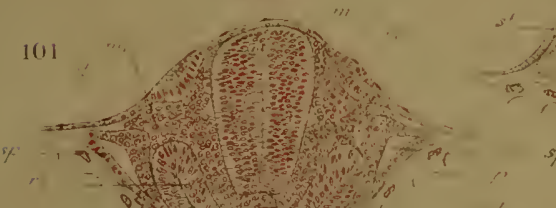

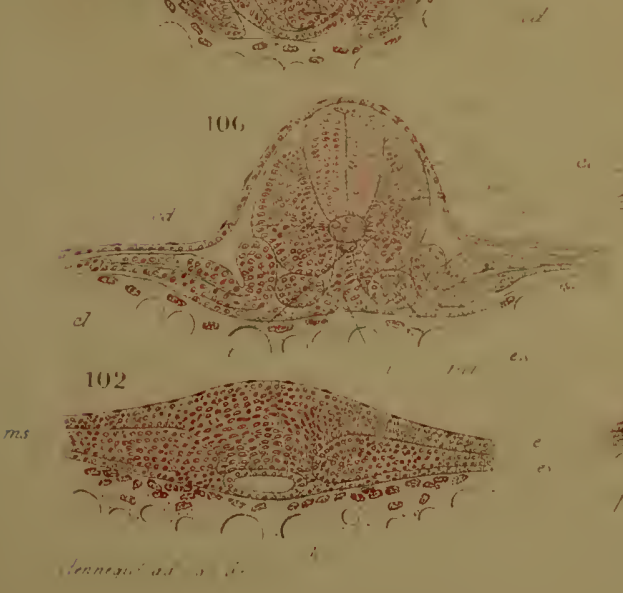

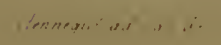

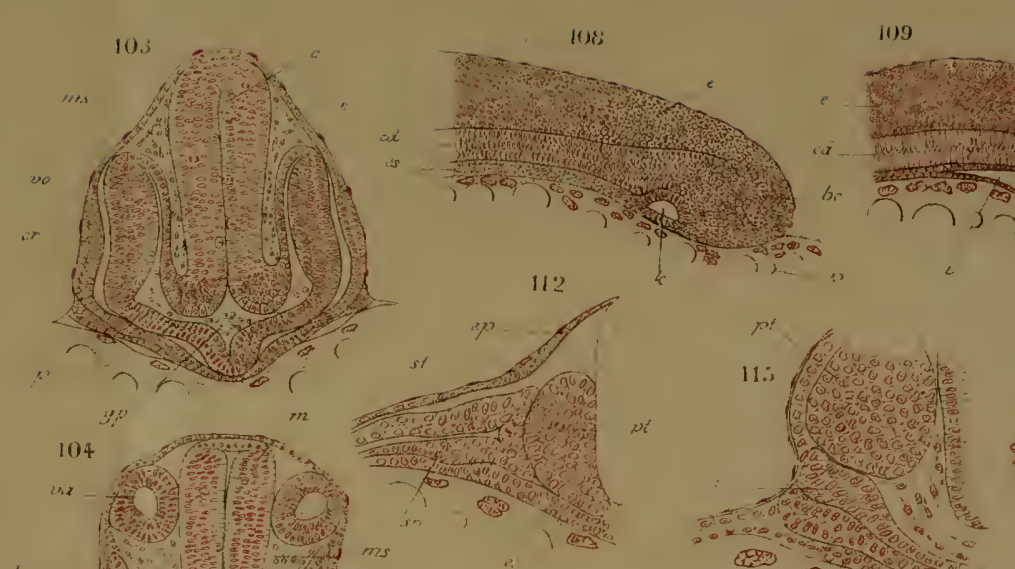

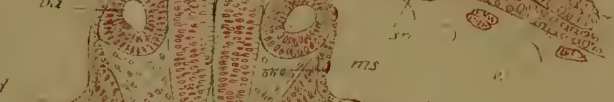

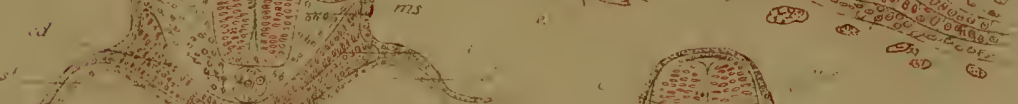

Af $+3$

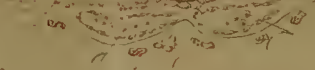

sp $p$
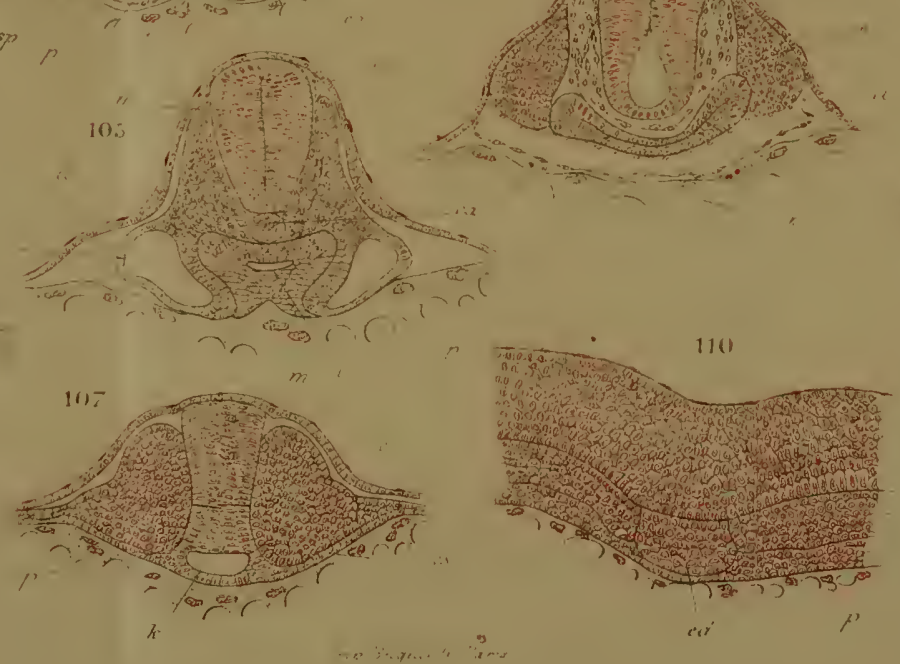

A.
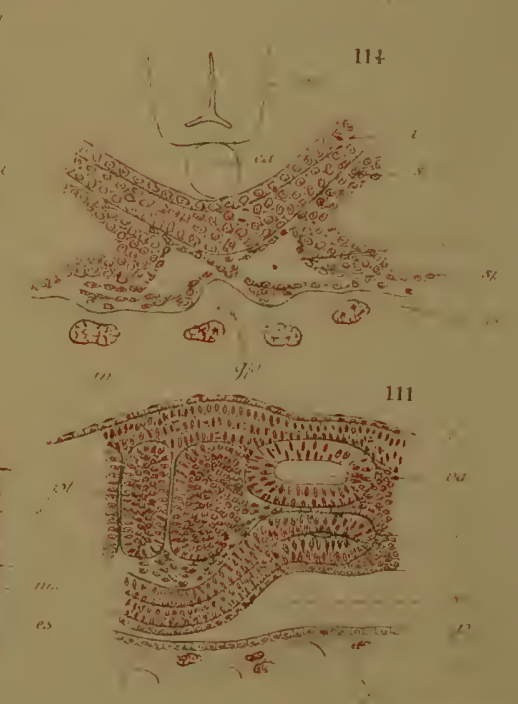

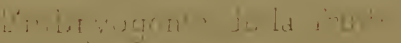

the 11 is 



(1)

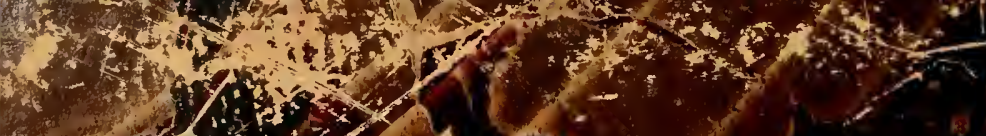

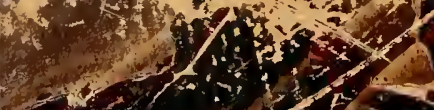

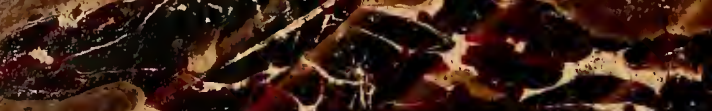

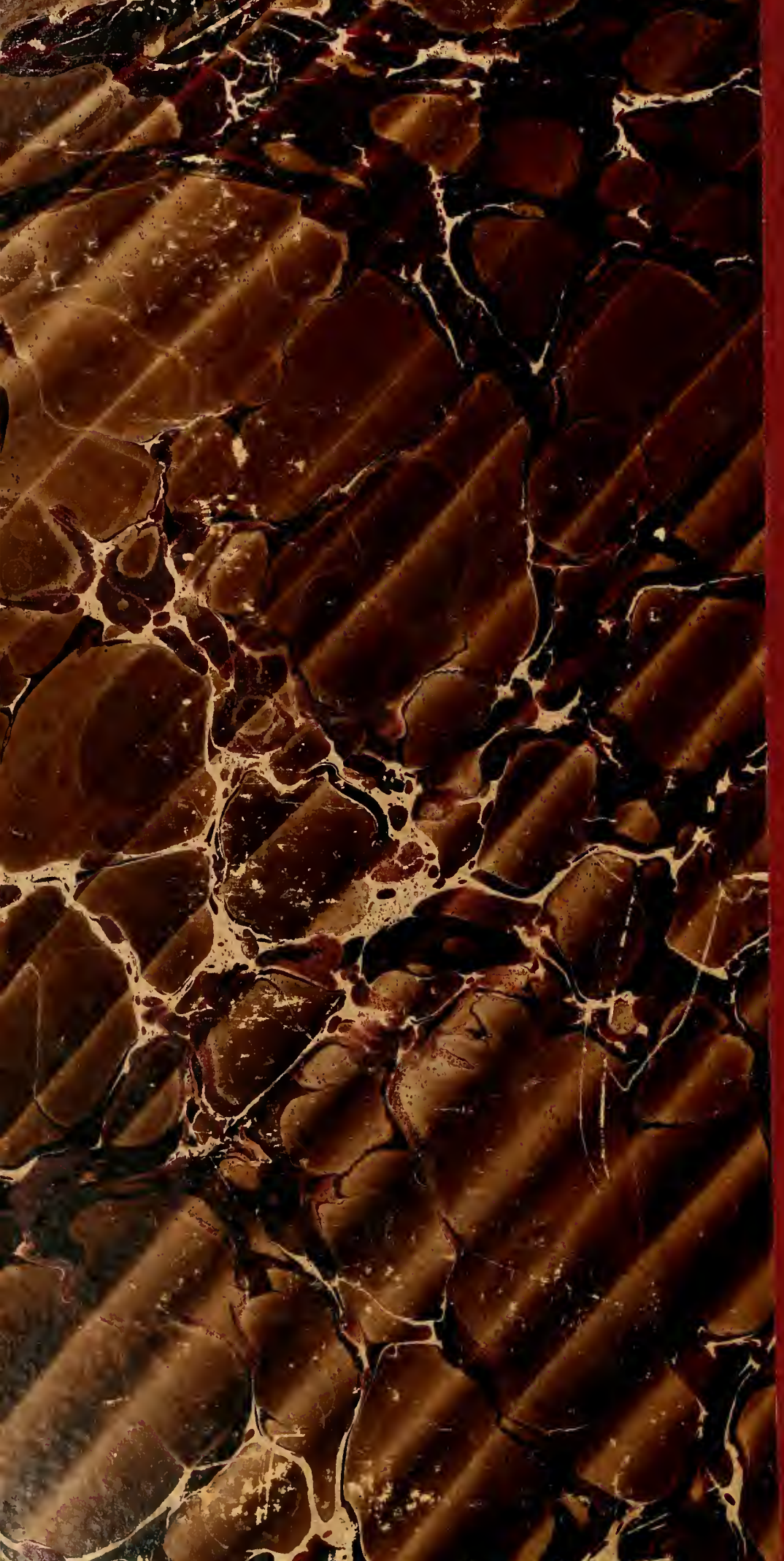

